\title{
JUDGEMENT POST-STRATIFICATION FOR DESIGNED EXPERIMENTS
}

\section{DISSERTATION}

\author{
Presented in Partial Fulfillment of the Requirements for \\ the Degree Doctor of Philosophy in the \\ Graduate School of The Ohio State University \\ By \\ Juan Du, M.S. \\ $* * * * *$ \\ The Ohio State University \\ 2006
}

Dissertation Committee:

Approved by

Professor Steven N. MacEachern, Adviser

Professor Omer Ozturk

Professor Elizabeth Stasny

Professor Douglas Wolfe

Adviser

Graduate Program in Statistics 
(c) Copyright by

Juan Du

2006 


\begin{abstract}
In many scientific studies, information that is not easily translated into covariates is ignored in the analysis. This type of information, however, may significantly improve inference. In this research, we apply the idea of judgment post-stratification to utilize such information. Specifically, we consider experiments that are conducted under a completely randomized design. Sets of experimental units are formed, and the units in a set are ranked. Estimation is performed conditional on the sets and ranks. We propose a new estimator for a treatment contrast. We improve the new estimator by Rao-Blackwellization. Asymptotic distribution theory and corresponding inferential procedures for both estimators are developed. Simulation studies quantify the superiority of the new estimators and show their desirable properties for small and moderate sample sizes. An example from the design literature illustrates the success of the new techniques. We also develop permutation tests for the new estimators and compare them with the testing procedures based on asymptotic theory.
\end{abstract}


To my husband Yufeng and my son Andrew. 


\section{ACKNOWLEDGMENTS}

I express my sincere gratitude to my advisor Professor Steven N. MacEachern for his support, encouragement, and guidance. It has been a great pleasure to have Professor MacEachern as my supervisor. Without his help, this work could not have been completed. I would like to thank him for always being so supportive and encouraging concerning my work.

I wish to thank Professors Omer Ozturk, Elizabeth Stasny, and Douglas Wolfe for serving in my dissertation committee and their great comments and suggestions. I also want to thank many friends at Ohio State for their friendship.

Lastly but not the least, I thank my husband Yufeng and my parents for their love and support. 


\section{VITA}

\begin{tabular}{|c|c|}
\hline 1999 & $\begin{array}{l}\text { B.S. Economics, } \\
\text { Nankai University }\end{array}$ \\
\hline $2004 \ldots$ & $\begin{array}{l}\text { M.S. Statistics, } \\
\text { The Ohio State University }\end{array}$ \\
\hline 2003 & $\begin{array}{l}\text { Summer Intern, } \\
\text { Merkle Direct Marketing Inc. }\end{array}$ \\
\hline $2002-2005$ & $\begin{array}{l}\text { Graduate Teaching and Research Asso- } \\
\text { ciate, The Ohio State University }\end{array}$ \\
\hline 2005 - present & $\begin{array}{l}\text { Risk Software Tester, SAS Institute } \\
\text { Inc. }\end{array}$ \\
\hline
\end{tabular}

Major Field: Statistics 


\section{TABLE OF CONTENTS}

Page

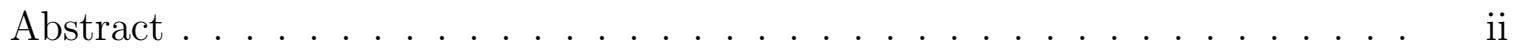

Dedication ...........................

Acknowledgments ....................... iv

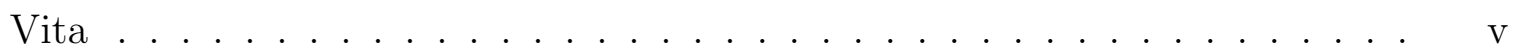

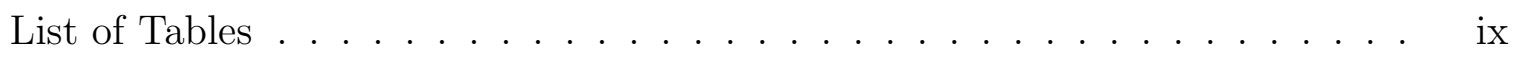

List of Figures $\ldots \ldots \ldots \ldots \ldots \ldots \ldots \ldots \ldots \ldots \ldots \ldots \ldots$ xiii

Chapters:

1. Introduction . . . . . . . . . . . . . . . . . 1

1.1 Ranked Set Sampling . . . . . . . . . . . . . . . . . . . 1

1.1.1 One Sample Problems . . . . . . . . . . . . . . . . . 2

1.1.2 Two and Multi-sample Problems . . . . . . . . . . 5

1.2 Order Restricted Randomized Designs . . . . . . . . . . . . . 7

1.3 Judgement Post-Stratification . . . . . . . . . . . . . . . . . . . 9

1.4 A Combination of ORR and JPS . . . . . . . . . . . . 12

2. The Judgement Post-Stratification Estimator . . . . . . . . . . . . 14

2.1 Randomization and Ranking Procedure . . . . . . . . . . . . 14

2.2 Models . . . . . . . . . . . . . . . . . . . . . . . 16

2.2 .1 Location-Scale Models . . . . . . . . . . . . . . . . 16

2.2 .2 Cell-Mean Models . . . . . . . . . . . . . . . . . . . . 19

2.3 The Judgement Post-Stratification Estimator . . . . . . . . . . 20

2.3.1 Properties of JPS Estimator . . . . . . . . . . . . . 21 
2.3.2 Comparison of The JPS and SRS Estimator . . . . . . . . . 24

2.3.3 Limiting Distribution and Inferential Procedures . . . . . . 28

2.4 Simulations ..................... . . . . 34

3. Rao-Blackwellized Estimators . . . . . . . . . . . . . . . . 62

3.1 The Rao-Blackwellization of the JPS Estimator . . . . . . . . . . . 62

3.1.1 The Rao-Blackwellized Estimator . . . . . . . . . . . . 63

3.1.2 The Vector Rao-Blackwellized Estimator . . . . . . . . . . . 64

3.1.3 An Illustrative Example for $\hat{\Delta}_{1}, \hat{\Delta}_{2}$, and $\hat{\Delta}_{3} \ldots . . . . . .666$

3.2 Limiting Distribution of The VRBE . . . . . . . . . . . . . . . . . 69

3.3 Inferential Procedures of the VRBE . . . . . . . . . . . . . . 71

3.4 An Illustration with a Two-Sample T-Test . . . . . . . . . . . . . . 74

3.5 The Example in Section 3.1.3 Continued . . . . . . . . . . . . . . . 77

3.6 Simulations . . . . . . . . . . . . . . . . . 79

3.7 Unbalanced, Randomized Two-Sample Designs . . . . . . . . . . . 81

3.7.1 $\hat{\Delta}_{3}$ for Unbalanced, Randomized Two-Sample Designs . . . 82

3.7.2 $\hat{\Delta}_{1}$ for Unbalanced, Randomized Two-Sample Designs . . . 86

4. Permutation Tests . . . . . . . . . . . . . . . . 97

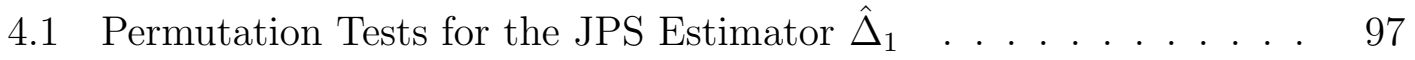

4.1.1 Procedure . . . . . . . . . . . . . . . . . 97

4.1.2 Properties.......................... 102

4.1.3 Extensions of The Permutation Tests . . . . . . . . . . . 106

4.1 .4 Confidence Intervals . . . . . . . . . . . . . . . . . . . . . 107

4.2 A Permutation Test for The VRBE $\hat{\Delta}_{3} \ldots \ldots$. . . . . . . . . . . 109

4.3 Simulations ....................... . . . 111

5. Latex Balloon Manufacture . . . . . . . . . . . . . . . 126

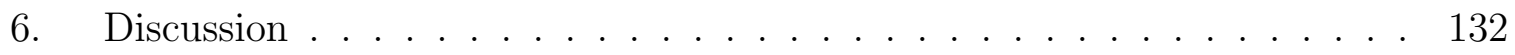

6.1 Conclusions . . . . . . . . . . . . . . . . . . . . . . . . 132

6.2 Future Directions . . . . . . . . . . . . . . . . . 134

Appendices:

A.

A.1 Comparing $\hat{\Delta}, \hat{\Delta}_{1}, \hat{\Delta}_{2}$, and $\hat{\Delta}_{3} \ldots \ldots \ldots \ldots$. . . . . . . . 136 
A.2 Calculating Estimated Levels for Test Statistics based on Asymptotic Theory. . . . . . . . . . . . . . . . . . 139

Bibliography . . . . . . . . . . . . . . . . . . . . . 144 


\section{LIST OF TABLES}

Table

Page

2.1 Judgement post-stratification for a balanced, randomized two-sample design. Each row of the table gives a set. After reordering the sets, the first $n_{1}$ are Type 1 sets; the remaining $n-n_{1}$ are Type 2 sets. Notation for the responses includes as a subscript treatment, rank and set number. . . . . . . . . . . . . . . . . .

2.2 Mean square errors and relative efficiency of the estimators $\hat{\Delta}_{1}$ and $\hat{\Delta}$. Standard errors are given in parentheses. The simulation study is based on 10,000 replicates, $\mu_{1}-\mu_{2}=-1$ and $\sigma_{1}=\sigma_{2}=1$. . . . .

2.3 Estimated levels of a nominal $\alpha=0.05$ test for Student's $t$-distribution $\left(T_{n}\right)$ with $n-2$ d.f., Limiting normal distribution $\left(Z_{n}\right)$, Satterthwaite's approximation $\left(S_{n}\right)$ for $\hat{\Delta}_{1}$, and Student's $t$-distribution $\left(T_{n}^{S R S}\right)$ with $2 n-2$ d.f. for $\hat{\Delta}$. The simulation study is based on 10,000 replicates, $\sigma_{1}=\sigma_{2}=1$. Standard errors are given in parentheses. . . . . . . . .

2.4 Estimated levels for a nominal level $\alpha=0.05$ test conditional on $n_{1}$ when sample size $n=10$. The simulation study is based on 10,000 replicates, $\sigma_{1}=\sigma_{2}=1$. Ranking quality varies from perfect ranking to random ranking. Standard errors are given in the parentheses. . .

2.5 Estimated levels for a nominal level $\alpha=0.05$ test conditional on grouped $n_{1}$ when sample size $n=20$. The simulation study is based on 10,000 replicates, $\sigma_{1}=\sigma_{2}=1$. Ranking quality varies from perfect ranking to random ranking. Standard errors are given in the parentheses. 51

2.6 Estimated levels for a nominal level $\alpha=0.05$ test conditional on grouped $n_{1}$ when sample size $n=100$. The simulation study is based on 10,000 replicates, $\sigma_{1}=\sigma_{2}=1$. Ranking quality varies from perfect ranking to random ranking. Standard errors are given in parentheses. 
2.7 Estimated levels of a nominal $\alpha=0.05$ test for Student's $t$-distribution $\left(T_{n}\right)$ with $n-2$ d.f., Limiting normal distribution $\left(Z_{n}\right)$ for $\hat{\Delta}_{1}$, and for Student's $t$-distribution $\left(T_{n}^{S R S}\right)$ with $2 n-2$ d.f. and approximate normal distribution $\left(Z_{n}^{S R S}\right)$ for $\hat{\Delta}$. Simulation is based on 10,000 replicates, scale parameters for the two treatments are set to 1 and 4 respectively. Standard errors are given in parentheses. . . . . . . . . . . . . . . .

2.8 Estimated levels of a nominal $\alpha=0.05$ test for Student's $t$-distribution $\left(T_{n}\right)$ with $n-2$ d.f., Limiting normal distribution $\left(Z_{n}\right)$ for $\hat{\Delta}_{1}$, and for Student's $t$-distribution $\left(T_{n}^{S R S}\right)$ with $2 n-2$ d.f. and approximate normal distribution $\left(Z_{n}^{S R S}\right)$ for $\hat{\Delta}$ under perfect ranking. Simulation is based on 10,000 replicates, $\sigma_{1}=\sigma_{2}$. The underlying distribution for $\epsilon$ is lognormal with mean of $\log (\epsilon)$ set to 0 and standard deviation of $\log (\epsilon)$ to be $0.5,1,2,4$ respectively. Standard errors are given in

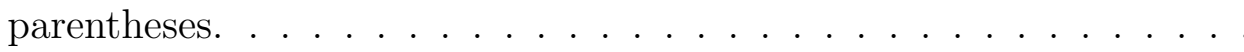

2.9 Estimated level of a nominal $\alpha=0.05$ test for Student's $t$-distribution $\left(T_{n}\right)$ with $n-2$ d.f., Limiting normal distribution $\left(Z_{n}\right)$ for $\hat{\Delta}_{1}$, and for Student's $t$-distribution $\left(T_{n}^{S R S}\right)$ with $2 n-2$ d.f. and approximate normal distribution $\left(Z_{n}^{S R S}\right)$ for $\hat{\Delta}$ under perfect ranking. Simulation is based on 10,000 replicates, $\sigma_{1}=\sigma_{2}$. The underlying distributions for $\epsilon$ are $t$ with degree of freedom $5,10,20,40$. Standard errors are given in parentheses. . . . . . . . . . . . . . . . .

3.1 Mean square errors of the estimators $\hat{\Delta}_{1}, \hat{\Delta}_{2}, \hat{\Delta}_{3}$, and $\hat{\Delta}$. Standard errors are given in the parentheses. The simulation study is based on 10000 replicates, $\mu_{1}-\mu_{2}=-1$ and $\sigma_{1}=\sigma_{2}=1$. . . . . . . . .

3.2 One possible arrangements of the data with sample size equal to 4 and the rankings for the four pairs are $\left\{u_{11}<u_{21} ; u_{12}>u_{22}, u_{13}<\right.$ $\left.u_{23} ; u_{14}>u_{24}\right\} \ldots \ldots \ldots \ldots \ldots \ldots$

3.3 Estimated levels of a nominal $\alpha=0.05$ test for Student's $t$-distribution $\left(T_{n}\right)$ with $n-2$ d.f., the Limiting normal distribution $\left(Z_{n}\right)$, and Satterthwaite's approximation $\left(S_{n}\right)$ for $\hat{\Delta}_{1}$, Student's $t$-distribution $\left(T_{n}^{S R S}\right)$ with $2 n-2$ d.f. and the normal distribution $\left(Z_{n}^{S R S}\right)$ for $\hat{\Delta}$, the Limiting normal distribution $\left(Z_{n}^{3}\right)$ and Student's $t$-distribution $\left(T_{n}^{3}\right)$ with $n-1$ d.f. for $\hat{\Delta}_{3}$. Simulations are based on 10,000 replicates, scale parameters are set to 1 . Standard errors range from 0.001 to 0.003 . . 
3.4 Estimated levels of a nominal $\alpha=0.05$ test for Student's $t$-distribution $\left(T_{n}\right)$ with $n-2$ d.f., the Limiting normal distribution $\left(Z_{n}\right)$, and Satterthwaite's approximation $\left(S_{n}\right)$ of $\hat{\Delta}_{1}$, Student's $t$-distribution $\left(T_{n}^{S R S}\right)$ with $2 n-2$ d.f. and the normal distribution $\left(Z_{n}^{S R S}\right)$ of $\hat{\Delta}$, the Limiting normal distribution $\left(Z_{n}^{3}\right)$ and Student's $t$-distribution $\left(T_{n}^{3}\right) n-1$ d.f. of $\hat{\Delta}_{3}$. Simulations are based on 10,000 replicates, $\sigma_{1}$ and $\sigma_{2}$ are set to 1 and 4, respectively. Standard errors range from 0.002 to 0.003 . . . .

3.5 Estimated levels of a nominal $\alpha=0.05$ test for Student's $t$-distribution $\left(T_{n}\right)$ with $n-2$ d.f., Limiting normal distribution $\left(Z_{n}\right)$ and Satterthwaite's approximation $\left(S_{n}\right)$ of $\hat{\Delta}_{1}$, Student's $t$-distribution $\left(T_{n}^{S R S}\right)$ with $2 n-2$ d.f. and the Limiting normal distribution $\left(Z_{n}^{S R S}\right)$ of $\hat{\Delta}$, the Limiting normal distribution $\left(Z_{n}^{3}\right)$ and Student's $t$-distribution $\left(T_{n}^{3}\right)$ with $n-1$ d.f. of $\hat{\Delta}_{3}$. Simulations are based on 10,000 replicates, $\sigma_{1}=\sigma_{2}=1$. The underlying distribution for $\epsilon$ is lognormal with mean of $\log (\epsilon)$ set to 0 and standard deviation of $\log (\epsilon)$ set to $0.5,1,2$, and 4. Standard errors range from 0.001 to 0.003 .

3.6 Estimated levels of a nominal $\alpha=0.05$ test for Student's $t$-distribution $\left(T_{n}\right)$ with $n-2$ d.f., Limiting normal distribution $\left(Z_{n}\right)$ and Satterthwaites approximation $\left(S_{n}\right)$ of $\hat{\Delta}_{1}$, Student's $t$-distribution $\left(T_{n}^{S R S}\right)$ with $2 n-2$ d.f. and the Limiting normal distribution $\left(Z_{n}^{S R S}\right)$ of $\hat{\Delta}$, and the Limiting normal distribution $\left(Z_{n}^{3}\right)$, Student's $t$-distribution $\left(T_{n}^{3}\right)$ with $n-1$ d.f. of $\hat{\Delta}_{3}$. Simulations are based on 10,000 replicates, $\sigma_{1}=\sigma_{2}=1$. The underlying distributions for $\epsilon$ are $\mathrm{t}$ with d.f. 5, 10, 20 , and 40. Standard errors range from 0.001 to 0.003 . . . . . . .

4.1 Estimated levels of a nominal level $\alpha=0.05$ test based on Student's $t$-distribution $\left(T_{n}\right)$ with $n-2$ d.f. and the permutation test based on $\hat{\Delta}_{1}$. The simulation is based on 10,000 replicates. The underlying distribution of $\epsilon$ is $N(0,1), \sigma_{1}=\sigma_{2}=1$. Standard errors are given in parentheses. . . . . . . . . . . . . . . . .

4.2 Estimated levels of a nominal level $\alpha=0.05$ test for Student's $t$ distribution $\left(T_{n}\right)$ with $n-2$ d.f. and permutation test based on $\hat{\Delta}_{1}$. The simulation is based on 10,000 replicates. The underlying distribution of $\epsilon$ is $N(0,1), \sigma_{1}=1$, and $\sigma_{2}=4$. Standard errors are given in parentheses. . . . . . . . . . . . . . . . 
4.3 Estimated levels of a nominal level $\alpha=0.05$ test for Student's $t$ distribution $\left(T_{n}\right)$ with $n-2$ d.f. and permutation test based on $\hat{\Delta}_{1}$. The simulation is based on 10,000 replicates, $\sigma_{1}=\sigma_{2}$. The underlying distribution for $\epsilon$ is $t$-distribution with d.f. being $5,10,20$, and 40 . Standard errors are given in parentheses. . . . . . . . . . . . . . 120

4.4 Estimated levels of a nominal level $\alpha=0.05$ test for Student's $t$ distribution $\left(T_{n}\right)$ with $n-2$ d.f. and permutation test based on $\hat{\Delta}_{1}$. The simulation is based on 10,000 replicates, $\sigma_{1}=\sigma_{2}$. The underlying distribution for $\epsilon$ is lognormal with mean of $\log (\epsilon)$ to be 0 and standard deviation of $\log (\epsilon)$ to be $0.5,1,2,4$ respectively. Standard errors are given in parentheses. . . . . . . . . . . . . . . . . . . . . 121

5.1 Times (in seconds) for the balloon experiment, where the codes 1, 2, 3,4 denote the color pink, vellow, orange and blue. . . . . . . . . . .

5.2 Pairwise comparisons for four methods including the SRS estimator $\hat{\Delta}$, ANCOVA $\hat{\Delta}_{A N C O V A}$, JPS estimator $\hat{\Delta}_{1}$ and VRBE $\hat{\Delta}_{3}$, where the codes $1,2,3,4$ denote the colors pink, yellow, orange and blue, respectively. . . . . . . . . . . . . . . . . . . . . . . 130

5.3 Pairwise comparisons of the inflation times for the four colors using the permutation test. . . . . . . . . . . . . . . 131 


\section{LIST OF FIGURES}

Figure

Page

2.1 Comparison of the mean square errors both from unconditional and conditional point of view for $\hat{\Delta}_{1}$ with symbols 1 and 2 , and $\hat{\Delta}$ with symbols 3 and 4 . The sample size is 10 and ranking is perfect. . . . .

2.2 Normal Probability plots of $\hat{\Delta}_{1}$ and $\hat{\Delta}$ for perfect ranking when $n=10 \quad 40$

2.3 Normal Probability plots of $\hat{\Delta}_{1}$ and $\hat{\Delta}$ for perfect ranking when $n=10$ and $n_{1}=1,2,3 \ldots \ldots \ldots$. . . . . . . . . . . . . . . . . 41

2.4 Normal Probability plots of $\hat{\Delta}_{1}$ and $\hat{\Delta}$ for perfect ranking when $n=10$ and $n_{1}=4,5,6 \ldots \ldots \ldots \ldots \ldots \ldots$

2.5 Normal Probability plots of $\hat{\Delta}_{1}$ and $\hat{\Delta}$ for perfect ranking when $n=10$ and $n_{1}=7,8,9 \ldots \ldots \ldots \ldots \ldots \ldots$

2.6 Box-plots of $\hat{\Delta}_{1}$ and $\hat{\Delta}$ for selected $n_{1}$ under perfect ranking . . . . . 44

2.7 Box-plots of $\hat{\Delta}_{1}$ and $\hat{\Delta}$ for selected $n_{1}$ and $\tau^{2}=1 \ldots \ldots . . . . . \quad 45$

2.8 Box-plots of $\hat{\Delta}_{1}$ and $\hat{\Delta}$ for selected $n_{1}$ and $\tau^{2}=3 \ldots \ldots . . . . .46$

2.9 Box-plots of $\hat{\Delta}_{1}$ and $\hat{\Delta}$ for selected $n_{1}$ and $\tau^{2}=10000 \ldots \ldots$. . . . 47

2.10 Estimated levels of a nominal level $\alpha=0.05$ test for $T_{n}$, Student's $t$-distribution with $n-2$ d.f. and for $T_{n}^{S R S}$, Student's $t$-distribution with $2 n-2$ d.f. conditional on $n_{1}$. Sample size $n=10$. . . . . .

2.11 Empirical power of $T_{n}$ with $n-2$ d.f. (with symbol 1) and $T_{n}^{S R S}$ with $2 n-2$ d.f. (with symbol 2) under perfect ranking. . . . . . . . 54 
2.12 Empirical power of $T_{n}$ with $n-2$ d.f. (with symbol 1) and $T_{n}^{S R S}$ with $2 n-2$ d.f. (with symbol 2) when $\tau^{2}=1 . \ldots \ldots \ldots$

2.13 Empirical power of $T_{n}$ with $n-2$ d.f. (with symbol 1) and $T_{n}^{S R S}$ with $2 n-2$ d.f. (with symbol 2) when $\tau^{2}=3 \ldots \ldots \ldots$. . . . . .

2.14 Empirical power of $T_{n}$ with $n-2$ d.f. (with symbol 1 ) and $T_{n}^{S R S}$ with $2 n-2$ d.f. (with symbol 2 ) when $\tau^{2}=10,000 \ldots \ldots \ldots$. . . .

2.15 Empirical power of test statistics for $\hat{\Delta}_{1}$ including $t$-distribution with $n-2$ d.f. and normal approximation (indicated by labels 1 and 3 respectively) and test statistics for $\hat{\Delta}$ including $t$-distribution with $2 n-2$ d.f. and normal approximation (indicated by labels 2 and 4 respectively) under perfect ranking. The simulation is based on 10,000 replicates. Scale parameters for the two treatments are set to be 1 and 4 respectively. 59

3.1 Normal Probability plots of $\hat{\Delta}, \hat{\Delta}_{1}$, and $\hat{\Delta}_{3}$ for perfect ranking when

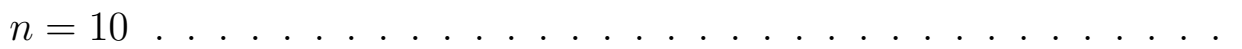

3.2 Empirical power of $T_{n}$ for $\hat{\Delta}_{1}$ with symbol $1, T_{n}^{S R S}$ for $\hat{\Delta}$ with symbol $2, Z_{n}^{3}$ and $T_{n}^{3}$ for $\hat{\Delta}_{3}$ with symbols 3 and 4 , respectively, under perfect ranking. . . . . . . . . . . . . . . . . .

3.3 Empirical power of $T_{n}$ for $\hat{\Delta}_{1}$ with symbol $1, T_{n}^{S R S}$ for $\hat{\Delta}$ with symbol $2, Z_{n}^{3}$ and $T_{n}^{3}$ for $\hat{\Delta}_{3}$ with symbols 3 and 4 , respectively, when $\tau^{2}=1$.

3.4 Empirical power of $T_{n}$ for $\hat{\Delta}_{1}$ with symbol $1, T_{n}^{S R S}$ for $\hat{\Delta}$ with symbol $2, Z_{n}^{3}$ and $T_{n}^{3}$ for $\hat{\Delta}_{3}$ with symbols 3 and 4 , respectively, when $\tau^{2}=3$.

3.5 Empirical power of $T_{n}$ for $\hat{\Delta}_{1}$ with symbol $1, T_{n}^{S R S}$ for $\hat{\Delta}$ with symbol 2, $Z_{n}^{3}$ and $T_{n}^{3}$ for $\hat{\Delta}_{3}$ with symbols 3 and 4 , respectively, when $\tau^{2}=10000$.

3.6 Empirical power of test statistics for $\hat{\Delta}_{1}$ including the $t$-distribution with $n-2$ d.f. and the normal approximation (indicated by labels 1 and 3 respectively) and test statistics for $\hat{\Delta}$ including the $t$-distribution with $2 n-2$ d.f. and the normal approximation (indicated by labels 2 and 4 respectively), and test statistics for $\hat{\Delta}_{3}$ including the normal approximation and the $t$-distribution with $n-1$ d.f. (indicated by labels 5 and 6 respectively) under perfect ranking. The simulations are based on 10,000 replicates. $\sigma_{1}$ and $\sigma_{2}$ are set to 1 and 4 , respectively. . . . 
4.1 Empirical power of $T_{n}$ with $n-2$ d.f. with label 1 and permutation test based on $\hat{\Delta}_{1}$ with label 2 for perfect ranking. The simulation is based on 10,000 replicates. The underlying distribution of $\epsilon$ is $N(0,1)$, $\sigma_{1}=\sigma_{2}=1 \ldots \ldots \ldots \ldots \ldots \ldots \ldots \ldots \ldots \ldots \ldots \ldots \ldots \ldots$

4.2 Empirical power of $T_{n}$ with $n-2$ d.f. with label 1 and permutation test based on $\hat{\Delta}_{1}$ with label 2 for $\tau=1$. The simulation is based on 10,000 replicates. The underlying distribution of $\epsilon$ is $N(0,1), \sigma_{1}=\sigma_{2}=1$.

4.3 Empirical power of $T_{n}$ with $n-2$ d.f. with label 1 and permutation test based on $\hat{\Delta}_{1}$ with label 2 for $\tau=\sqrt{3}$. The simulation is based on 10,000 replicates. The underlying distribution of $\epsilon$ is $N(0,1), \sigma_{1}=\sigma_{2}=1$.

4.4 Empirical power of $T_{n}$ with $n-2$ d.f. with label 1 and permutation test based on $\hat{\Delta}_{1}$ with label 2 for $\tau=100$. The simulation is based on 10,000 replicates. The underlying distribution of $\epsilon$ is $N(0,1), \sigma_{1}=\sigma_{2}=1$.

4.5 Empirical power of $T_{n}$ with $n-2$ d.f. with label 1 and permutation test based on $\hat{\Delta}_{1}$ with label 2 for perfect ranking. The simulation is based on 10,000 replicates. The underlying distribution for $\epsilon$ is lognormal with mean of $\log (\epsilon)$ to be 0 and standard deviation of $\log (\epsilon)$ to be 0.5 , $\sigma_{1}=\sigma_{2}=1 \ldots \ldots \ldots \ldots \ldots \ldots \ldots \ldots \ldots \ldots \ldots \ldots \ldots$

4.6 Empirical power of $T_{n}$ with $n-2$ d.f. with label 1 and permutation test based on $\hat{\Delta}_{1}$ with label 2 for perfect ranking. The simulation is based on 10,000 replicates. The underlying distribution for $\epsilon$ is lognormal with mean of $\log (\epsilon)$ to be 0 and standard deviation of $\log (\epsilon)$ to be 1 , $\sigma_{1}=\sigma_{2}=1 \ldots \ldots \ldots \ldots \ldots \ldots \ldots \ldots \ldots \ldots \ldots \ldots \ldots \ldots$

4.7 Empirical power of $T_{n}$ with $n-2$ d.f. with label 1 and permutation test based on $\hat{\Delta}_{1}$ with label 2 for perfect ranking. The simulation is based on 10,000 replicates. The underlying distribution for $\epsilon$ is lognormal with mean of $\log (\epsilon)$ to be 0 and standard deviation of $\log (\epsilon)$ to be 2, $\sigma_{1}=\sigma_{2}=1 \ldots \ldots \ldots \ldots \ldots \ldots \ldots$

4.8 Empirical power of $T_{n}$ with $n-2$ d.f. with label 1 and permutation test based on $\hat{\Delta}_{1}$ with label 2 for perfect ranking. The simulation is based on 10,000 replicates. The underlying distribution for $\epsilon$ is lognormal with mean of $\log (\epsilon)$ to be 0 and standard deviation of $\log (\epsilon)$ to be 4 , $\sigma_{1}=\sigma_{2}=1 \ldots \ldots \ldots \ldots \ldots \ldots \ldots \ldots \ldots \ldots \ldots \ldots \ldots$ 
5.1 Residual plot for analysis of covariance model. . . . . . . . . . . . . 129 


\section{CHAPTER 1}

\section{INTRODUCTION}

For a completely randomized design, a standard estimator for the contrast parameter in two sample problems, $\Delta=\mu_{1}-\mu_{2}$, is the difference of the two sample means, i.e. $\hat{\Delta}=\bar{Y}_{1}-\bar{Y}_{2}$. In many scientific studies, however, a different type of information is available on experimental units (EU). Some information can be easily quantified, while other information is difficult to describe. For information that can be directly translated into a small number of covariates, traditional techniques such as Analysis of Covariance (ANCOVA) can be applied to improve accuracy of the inference. The information that can not be easily used, however, has been typically ignored during the design and analysis of experiments.

\subsection{Ranked Set Sampling}

Ranked set sampling (RSS), first proposed by McIntyre $(1952,2005)$ as an alternative method of simple random sampling to improve the accuracy of the estimation of mean pasture and forage yield, exploits some of this difficult-to-quantify information to alter the data collection procedure. It is most useful in situations where the exact measurement or quantification of sample units is difficult or expensive in terms of time or money, but where a small set of sample units can be easily ranked with 
reasonable accuracy through subjective judgement or via the use of a concomitant variable correlated with the characteristic of interest.

After McIntyre's original work, RSS has been intensively studied, often in agricultural and environmental applications. See Halls and Dell (1966) and Evans (1967) for early examples, Ridout and Cobby (1987), Kaur et al. (1995), Barnett (1999), and Mode et al. (1999) for review and recent examples.

\subsubsection{One Sample Problems}

In an infinite population setting, RSS is similar to stratified sampling with the ranks serving as the strata. Instead of creating strata before the data collection, however, one creates the strata while collecting the data. In its original form, the procedure of selecting a ranked set sample in a one sample problem involves drawing $m$ random samples from an infinite population with $m$ units in each sample. Here $m$ denotes the set size and is a design parameter to be chosen by the investigator. Within each set, the $m$ units are ranked from the smallest to the largest by visual inspection or some rough method without requiring detailed measurements. From the first set, the unit corresponding to the smallest rank is selected to be fully measured. From the second set, take the unit with the second smallest rank. This process continues until we have selected $m$ units for full measurement. In this procedure, although we have $m^{2}$ sample units, we only choose $m$ of them to be quantified. This sampling procedure with one cycle is illustrated by reproducing the Figure 1.1 in Chen et al. (2003) as follows:

$$
\begin{aligned}
& \mathbf{Y}_{\mathbf{1}[\mathbf{1}]} \leq Y_{1[2]} \leq \cdots \leq Y_{1[m]} \quad \rightarrow \quad \mathbf{Y}_{[\mathbf{1}]} \\
& Y_{2[1]} \leq \mathbf{Y}_{\mathbf{2}[\mathbf{2}]} \leq \cdots \leq Y_{2[m]} \quad \rightarrow \quad \mathbf{Y}_{[\mathbf{2}]} \\
& \begin{array}{cccccc}
\vdots & \vdots & \vdots & \vdots & \vdots & \vdots \\
Y_{m[1]} \leq & Y_{m[2]} \leq & \cdots & \mathbf{Y}_{\mathbf{m}[\mathbf{m}]} & \rightarrow & \mathbf{Y}_{[\mathbf{m}]},
\end{array}
\end{aligned}
$$


where $\mathbf{Y}_{[\mathbf{1}]}, \mathbf{Y}_{[\mathbf{2}]}, \ldots, \mathbf{Y}_{[\mathbf{m}]}$ are the units selected for full measurement. These selected units are from different samples, so they are independent. They also include each rank position from the smallest to the largest. To get enough samples for inference, the entire procedure can be replicated $k$ times, which yields $n=m k$ fully measured units. These $n$ quantified units constitute the ranked set sample. Since there is an equal number of units selected from each rank, this procedure is called balanced ranked set sample (BRSS).

A more general RSS protocol can be found in Dell and Clutter (1972). Different from the sequential scheme initially used to introduce the ranked set concept, this procedure use a restricted-random approach to obtain unequal but specified allocation. It establishes a one-to-one association between the elements of the set and the $m$ ranks and partitions the population into $m$ equal parts. This process is repeated for these $n$ sets and produce $n_{1}$ quantified elements from judgement rank $1, n_{2}$ elements from rank $2, \cdots$, and $n_{m}$ from judgement rank $m$, where $n=n_{1}+n_{2}+\cdots+n_{m}$. In general, the values of $n_{1}, \cdots, n_{m}$ are predetermined and the randomization process is controlled to produce the specified value of $n_{i}$. Let $Y_{i[h]} ; i=1,2, \cdots, n, h=1,2, \cdots, m$, denote the observation with judgement rank $h$ from the $i$-th sample. The ranked set sample can be represented as

$\begin{array}{cccc}Y_{1[1]} & Y_{2[1]} & \cdots & Y_{n_{1}[1]} \\ Y_{1[2]} & Y_{2[2]} & \cdots & Y_{n_{2}[2]} \\ \vdots & \vdots & \vdots & \vdots \\ Y_{1[m]} & Y_{2[m]} & \cdots & Y_{n_{m}[m]} .\end{array}$

In the situation where $n_{1}=n_{2}=\cdots=n_{m}=k$, the procedure yields a balanced ranked set sample. Otherwise, an unbalanced ranked set sample (URSS) is generated.

Because of the success of RSS on applications, Takahasi and Wakimoto (1968) started to investigate the theoretical properties. They showed that the estimator 
based on a balanced or unbalanced RSS, $\hat{\mu}=\frac{1}{m} \sum_{r=1}^{m} \bar{Y}_{[r]}$, where $\bar{Y}_{[r]}$ is the arithmetic mean of the $n_{r}$ measured values from the $r$-th rank i.e. $\frac{1}{n_{r}} \sum_{i=1}^{n_{r}} Y_{i[r]}$, is an unbiased estimator of population mean $\mu$. For a balanced RSS, the variance of this estimator is always no larger than the variance of the estimator based on simple random sample with the same sample size, with strict inequality holding if the means of the ranks differ. The later work of Dell and Clutter (1972) obtained a similar result under a more general situation. David and Levine (1972) and Bohn and Wolfe (1994) discussed the model of ranking errors. RSS can also be used to estimate variance (Stoke (1980), Sinha et al. (1996), MacEachern et al. (2002), and Perron and Sinha (2004)), and even the entire distribution function (Stokes and Sager (1988), Kvam and Samaniego (1993), and Chen (2000)).

In this dissertation, we use square brackets around subscripts for judgement ranked order statistics and save parentheses for the true order statistics. When the judgement ranked orders coincide with their numeric orders, then the ranking is said to be perfect. Otherwise, there is a difference between order statistics and judgement ranked order statistics, hence the ranking is imperfect. In the case of perfect ranking, where the judgement order statistics are the same as the order statistics, the ranked set sample is composed of independent order statistics, while for a ranked simple random sample, the order statistics are positively correlated. Thus, the source and amount of the improvement of RSS over SRS can be easily interpreted by the positive correlation among order statistics in a simple random sample (SRS). Consequently, those distributions that have more correlated order statistics will have the most significant improvement using RSS technique. More details can be found in Stokes and Sager (1988). 
The performance of RSS can be improved by allocating the sample size among the order statistics. Optimal allocation is discussed in Kaur et al. (1997), Barnett (1999), Nahhas (1999), Ozturk and Wolfe (2000a,c), Chen (2001), Nahhas et al. (2002), and Chen et al. (2005).

Although RSS usually yields more accurate inference than SRS, its performance can be affected by several factors: (i) Ranking error and the use of concomitant variable (Dell and Clutter (1972), David and Levine (1972), and Stokes (1977)). In the extreme case that the ranking is no better than random, the BRSS estimator will perform as well as the SRS estimator. (ii) Within set dependence (Ridout and Cobby (1987)). In the case of non-random selection of samples within sets, between sets variation is larger than variation within sets. The performance of ranked set sample will decrease as the ratio of between sets variance to within sets variance increases. (iii) The characteristic of the population under consideration (McIntyre (1952, 2005) and Husby et al. (2005)). As the skewness of the population increases, the performance of balanced ranked set sample diminishes. One can consider unbalance ranked set sampling, such as Neyman allocation, to further improve the performance of a RSS estimator. Inappropriate allocation may result in a relative precision less than one.

\subsubsection{Two and Multi-sample Problems}

In two sample problems, the ranked set sampling procedure can be described as follows: from the first population, choose $m$ random samples of size $m$ and rank each sample with respect to the perceived value of the response variable $Y$. Each of the $m$ samples is referred to as a set. In the first set, the unit with the smallest rank of 
$Y$ is selected for actual measurement. In the second set, the unit with the second smallest rank is measured, and so on, until, in the $m$-th set, the unit with the largest rank is measured. The entire procedure is replicated $k$ times yielding $m k$ measured units. This gives a BRSS from the first population. The same procedure is repeated for the second population to get another BRSS. These data yield estimates of the population means which are more accurate than those based on independent random samples from the two populations. This increase in accuracy results in more powerful hypothesis tests of testing difference in population means.

Two sample nonparametric testing procedures, such as the sign test and Mann Whitney-Wilcoxon rank sum test, are investigated by Bohn and Wolfe $(1992,1994)$, Ozturk (1999), Ozturk and Wolfe (2000b), and Fligner and MacEachern (2004). Parametric RSS with assumptions on the underlying distribution is also intensively studied by, for example, Fei et al. (1994), Stokes (1995), Sinha et al. (1996), Bhoj (1996, 1997), and Li and Chuiv (1997). Extensive work on designed experiments for RSS is developed in Muttlak (1996) for the one-way layout, Ozturk (2002) for rank regression in linear models, and Ozturk et al. (2004) for multi-sample inference.

Most of the above research makes no use of the randomization upon which designed experiments are based. For multi-sample problems, the research focuses on independent RSS. Another drawback of RSS is its need for a large number of experimental units. Compared to a simple random samples, a ranked set sample shows superiority with an equivalent number of quantified units in two sample problems, however, it requires a larger number of available experimental units than simple random sample does. For example, a balanced ranked set sample with set size $m$ requires $m^{2}$ experimental units although it only quantifies $m$ of them. The other $m(m-1)$ 
are discarded and only used to provide a context for the selected units. In a situation where available experimental units are rare or expensive, the cost of recruiting, ranking, and discarding a large number of units can be substantial.

\subsection{Order Restricted Randomized Designs}

To address these problems, Ozturk and MacEachern (2004, 2005) proposed the order restricted randomized (ORR) design. This design incorporates additional information about the EUs. In this method we group the units into sets at random, and then randomly assign some units in a set to one treatment and the remaining units to the second treatment. The ranking procedure creates positive correlations among each set of judgement ranked units. The assignment translates the positive correlations among units into a negative correlation for estimating the contrast between treatment means. Thus the variance of the sample contrast is reduced.

The randomization scheme can be summarized in the following two steps.

- Step 1: A set of $m$ experimental units is randomly selected and ranked from the smallest to the largest with respect to some overall quality measurement of each unit. Let $R_{1}, R_{2}, \cdots, R_{m}$ denote the ranks for the units, and the units can be divided into two disjoint sets. Denote the ranks for the units in the first set as $\alpha=\left\{R_{i_{1}}, \cdots, R_{i_{r}}\right\}$ and the ranks for the other set as $\beta=\left\{R_{j_{1}}, \cdots, R_{j_{m-r}}\right\}$, where $r$ is the largest integer less than or equal to $m / 2,\left\{i_{1}, \cdots, i_{r}\right\}$ is a subset of $\{1,2, \cdots, m\}$, and $\left\{j_{1}, \cdots, j_{m-r}\right\}$ is $\left\{i_{1}, \cdots, i_{r}\right\}^{C}$. A randomization is performed to assign the two treatments to the two different sets of units. 
- Step 2: Another set of $m$ experimental units is selected, ranked, and divided into two disjoint sets as in the first step. Without any randomization, the opposite allocation is performed for this set.

This procedure yields a balanced design since each treatment regime is assigned to every judgement ranked unit in the experiment. To generate enough experimental units for inference, the entire procedure can be repeated $n$ times to yield $n m$ observations for each treatment.

Based on the same estimator $\hat{\Delta}=\bar{X}-\bar{Y}$ as for the SRS, the variance of an ORR design involves positive covariances as well as negative ones. This variance can be minimized by choosing a design which has negative covariance as large as possible and positive covariance as small as possible among EUs. Equivalently, a design having two adjacent judgement ranked units assigned to different treatments will lead to an improvement of inference over that of RSS. Different from RSS, where the units are independent, an ORR design has positive correlation within each set. This positive correlation is translated into negative correlation when estimating the contrast parameter $\Delta=\mu_{1}-\mu_{2}$ leading to improved inference for the difference of two treatment means.

Ozturk and MacEachern $(2004,2005)$ compared the performance of the ORR with SRS and RSS theoretically as well as through a simulation study. Results show that the performance of an ORR design depends on $\alpha$. The optimal design can be obtained by distributing the elements of $\alpha$ and $\beta$ as evenly as possible. Ozturk and MacEachern (2005) also develop a test and corresponding confidence interval for the difference between two treatment means. They show that the Student $t$-distribution is a good approximation for the null distribution for sample size as small as $n=3$ 
and the proposed test outperforms its simple random sample and ranked set sample analogs.

\section{$1.3 \quad$ Judgement Post-Stratification}

Judgement post-stratification (JPS), based on an idea similar to RSS, was introduced by MacEachern et al. (2004). Similar to RSS, JPS also requires a set of random samples to be ranked using subjective judgement. This judgement process creates a covariate $R_{i}$ for each fully measured unit. It is the source of improvement in estimation precision both for RSS and JPS. Different from RSS, where the ranking information is used for collecting the data, however, JPS can be applied after the data are collected using SRS. This actually gives more flexibility to the investigator. The case of ties can be dealt with by considering imprecise rankings. Another distinct advantage of JPS is that it provides a mechanism for incorporating multiple rankers.

A judgement post-stratification sample with precise rankings can be summarized as follows:

- A random sample of $n$ units $Y_{1}, Y_{2}, \cdots, Y_{n}$ is selected from the population $F_{Y}$ in which we are interested. For each of the units $i ; i=1,2, \cdots, n$, another $H-1$ units are selected without exact measurement and just used to assign judgement rank to the fully measured unit.

- The $H$ units are ranked from the smallest to the largest and yield the judgement post-stratification sample which consists of $n$ pairs of $\left(Y_{i}, R_{i}\right)$, where $R_{i}$ denotes the rank for the fully measured unit.

In an infinite population setting, a RSS is a stratified sample where the ranks serve as the strata, while JPS is a post-stratified SRS, where each sample is post-stratified 
according to the ranks. Although most RSS assumes that the number of observations from each rank is predetermined, in the infinite population setting JPS coincides with a special RSS scheme, i.e. random allocation (Takahasi (1970)).

Suppose we are interested in estimating $\mu=E(Y)$, the population mean of $F_{Y}$ with a finite variance. In the precise ranking situation, let $R_{i}$ denote the rank for the fully measured unit and define $\mu_{h}=E\left(Y_{i} \mid R_{i}=h\right)$ and $\sigma_{h}^{2}=\operatorname{var}\left(Y_{i} \mid R_{i}=h\right)$ for $h=1,2, \cdots, H$, where $H$ is the number of ranks. The estimation of the population mean $\mu$ can be achieved by combining the estimator for each $\mu_{h}$, i.e. $\hat{\mu}=\frac{1}{H} \sum_{h=1}^{H} \hat{\mu}_{h}$. We define an indicator variable $I_{i h}=I\left(R_{i}=h\right)$ for each unit in every rank, then the RSS estimator $\hat{\mu}_{R S S}$ and the JPS estimator $\hat{\mu}_{J S}$ can be expressed in the same format:

$$
\frac{1}{H} \sum_{h=1}^{H} \frac{\sum_{i=1}^{n} Y_{i} I_{i h}}{\sum_{i=1}^{n} I_{i h}} .
$$

Notice that although the mean estimation has the same expression in both cases, BRSS specifies the number of the observations for each rank as $\frac{n}{H}$, while for the JPS, this number is a multinomial random variable with $n$ trials and probability $H^{-1}$.

The comparison of the two estimators is based on the variance of the limiting distribution as $n \rightarrow \infty$. For the BRSS estimator, we have

$$
\operatorname{var}\left(\hat{\mu}_{R S S}\right)=n^{-1} H^{-1} \sum_{h=1}^{H} \sigma_{h}^{2} .
$$

For JPS, there are two appropriate variances. One is from the unconditional point of view. Following Cochran (1977), this variance can be approximated by

$$
\operatorname{var}\left(\hat{\mu}_{J S}\right)=\operatorname{var}\left(\hat{\mu}_{R S S}\right)\left\{1+n^{-1}(H-1)\right\}
$$

The other variance conditions on the number of observations $n_{1}, n_{2}, \cdots, n_{H}$ for each rank and assume that all $n_{h}$ 's are positive. Then the variance is the same as that of 
an URSS, i.e.,

$$
\operatorname{var}\left(\hat{\mu}_{J S} \mid n_{1}, \cdots, n_{H}, \operatorname{alln}_{h}>0\right)=H^{-2} \sum_{h=1}^{H} \sigma_{h}^{2} n_{h}^{-1} .
$$

Note that as $n \rightarrow \infty$ and $n_{h} / n \rightarrow H^{-1}$, both variances of JPS estimators are asymptotically equivalent with that of the BRSS.

In the case of imprecise ranking, a distribution of the ranks, instead of a specific rank, is assigned to each observation. Define $p_{i}$ as a probability vector for the rank of a fully measured unit, i.e. $p_{i}=\left(p_{i 1}, p_{i 2}, \cdots, p_{i H}\right)$ and $p_{i h}=P\left(R_{i}=h\right)$. The JPS consists of a random sample of $\left(Y_{i}, p_{i}\right)$ 's. Three methods for estimating the population mean and corresponding estimators are introduced. The first one, called proportional proration, is based on a ratio of two quantities. Define $Z_{i h}=Y_{i} p_{i h}$ for all $i$ and $h$. An estimator of the population mean can be obtained as follows:

$$
\hat{\mu}_{1}=H^{-1} \sum_{h=1}^{H} \frac{\sum_{i=1}^{n} Z_{i h}}{\sum_{i=1}^{n} p_{i h}} .
$$

Instead of prorating an observation to several ranks, the second estimator randomly allocates the observation to a rank according to the probability $p_{i}$. Assuming no empty cells, the estimator is

$$
\hat{\mu}_{2}=H^{-1} \sum_{h=1}^{H} \frac{\sum_{i=1}^{n} Y_{i} I_{i h}}{\sum_{i=1}^{n} I_{i h}} .
$$

The third estimator is obtained by applying Rao-Blackwell Theorem on the second estimator, i.e., take the expectation of $\hat{\mu}_{2}$ over the distribution of $I_{i h}$. Thus,

$$
\hat{\mu}_{3}=E\left(\hat{\mu}_{2} \mid Y_{1}, \cdots, Y_{n}, p_{1}, \cdots, p_{n}\right) .
$$

The benefit of imprecise ranking is well-demonstrated by a simulation study performed by MacEachern et al. (2004) with $\hat{\mu}_{1}$ and $\hat{\mu}_{3}$ being the best performers. Variance estimation can be accomplished either through standard Taylor series expansion 
or with the random group method (see, for example, Lohr (1999)). A study on allometric data relating brain weight to body weight in different species of mammals shows that the Taylor series method can provide an accurate estimation of variance for $\hat{\mu}_{1}$ while the variance estimate conditioning on the observed $n_{h}$ is more appropriate for $\hat{\mu}_{2}$.

\subsection{A Combination of ORR and JPS}

Although an ORR can achieve improvement in precision and reduction in cost at the same time, there are cases where it is difficult to implement. To be specific, let us consider a clinical trial for two therapies, where patients accrue to the study through time. They are added to the experiment as they consent and will be assigned to one of the therapies at that time. Typically, a set of patients will not accrue at the same time. We could only implement ORR by delaying treatment for some patients - a practice we prefer to avoid. A completely randomized design may be a better choice in this instance. But after data collection, there is a wealth of information for the patients that cannot be used, including the medical history and some casual information that cannot be easily translated into covariates. To solve this problem, we borrow the idea of JPS and combine it with the ORR design. Under JPS, units are grouped into sets and ranked (thus creating strata defined by rank), and a random sample of units is chosen for measurement. To combine JPS and ORR post-experimentally, $n$ sets of units are randomly formed, with one unit from each treatment. The units are ranked only on the basis of pre-experimental information. This ranking is performed after the data are collected by completely randomized design. To make this methodology work well, good experimental technique is necessary. In particular, it is critical that 
the person who assigns ranks to experimental units should be "blinded" to the actual responses.

In this research, several new estimators for the treatment contrast are proposed. These new estimators are unbiased and have smaller variances than do the standard estimator, thus leading to superior inference.

The rest of this dissertation is organized as follows. In Chapter 2 we introduce the ranking procedure and the models, then propose a new estimator, the JPS estimator, for the contrast of treatments. We also develop the asymptotic distribution theory and corresponding inferential procedures for our new estimator. Finally, we present a numerical study for the comparison of the SRS and JPS estimators. In Chapter 3 we derive some estimators improved by Rao-Blackwellization and obtain the asymptotic normality theorem and corresponding inferential procedures. We discuss some randomization procedures and develop a randomization test in Chapter 4 and show the superiority of these new estimators to the SRS estimator through real examples in Chapter 5. We also discuss the relaxation of the conditioning to improve the tests. Concluding remarks are given in Chapter 6 . 


\section{CHAPTER 2}

\section{THE JUDGEMENT POST-STRATIFICATION ESTIMATOR}

\subsection{Randomization and Ranking Procedure}

For simplicity, we begin with two-sample problems, described as treatment versus control. We reserve subscripts 1 for the control group and subscripts 2 for the treatment group. For a balanced, completely randomized two-sample design, data are collected on $2 n$ experimental units. Among them, $n$ units are assigned to the treatment group and the other $n$ to the control group. The additional randomization and ranking is described in the following two steps.

- Step I: The $n$ units in the treatment group are randomly permuted. The $n$ units in the control group are randomly permuted. Grouping together the treatment and control units in the same positions creates $n$ sets, each consisting of two units.

- Step II: A qualified ranker is asked to assign ranks to all units in each pair as rank 1 and rank 2 based on a forecast response of the unit. A rank of 2 suggests a greater response than does a rank of 1 . These forecasts are made without knowledge of treatment assignment or the units' response. 
With appropriate "blinding", Steps I and II can be conducted post-experimentally just as well as pre-experimentally. In the context of the motivating medical study, the ranker can be a doctor who bases his/her forecasts on the medical histories of the patients (prior to enrollment in the study). The $n$ sets can be divided into two types. Type 1 sets consist of those pairs (sets) where the unit with rank 1 is assigned to the control and the unit with rank 2 assigned to the treatment. Type 2 sets are those for which the unit with rank 1 is assigned to the treatment. Let $n_{1}$ be the number of Type 1 sets and $n_{2}=n-n_{1}$ be the number of Type 2 sets. The random variable $n_{1}$ has a $\operatorname{Bin}(n, 0.5)$ distribution.

The following remarks provide some important features of the data:

- By design, we have same number of units for the treatment group as for the control group. We are likely to have different numbers of units for each rank within the treatment group and within the control group.

- The observations in a set are not independent. The process of forming a set and ranking the units produces dependence. Under minimal assumptions, the responses in a set are positively correlated.

- Observations in different sets are independent.

The data can be laid out as in Table 2.1. Let $R_{i}$ denote an indicator variable for each pair $\left(Y_{1 i}, Y_{2 i}\right)$ and let $S_{n}$ be the collection of sets of Type 1, where

$$
R_{i}= \begin{cases}1 & \text { when }\left(Y_{1 i}, Y_{2 i}\right) \in S_{n} \\ 0 & \text { when }\left(Y_{1 i}, Y_{2 i}\right) \notin S_{n},\end{cases}
$$

and $i=1, \cdots, n$. As for JPS in the one-sample problem, this covariate can be used to improve the precision of inference. 


\begin{tabular}{ccccccc}
\hline Set & Rank & Treatment & Response & Rank & Treatment & Response \\
\hline 1 & 1 & 1 & $Y_{1[1] 1}$ & 2 & 2 & $Y_{2[2] 1}$ \\
2 & 1 & 1 & $Y_{1[1] 2}$ & 2 & 2 & $Y_{2[2] 2}$ \\
$\cdot$ & $\cdot$ & $\cdot$ & $\cdot$ & $\cdot$ & $\cdot$ & $\cdot$ \\
$\cdot$ & $\cdot$ & $\cdot$ & $\cdot$ & $\cdot$ & $\cdot$ & $\cdot$ \\
$\cdot$ & $\cdot$ & $\cdot$ & $\cdot$ & $\cdot$ & $\cdot$ & $\cdot$ \\
$n_{1}$ & 1 & 1 & $Y_{1[1] n_{1}}$ & 2 & 2 & $Y_{2[2] n_{1}}$ \\
$n_{1}+1$ & 2 & 1 & $Y_{1[2] n_{1}+1}$ & $\cdot$ & 2 & $Y_{2[1] n_{1}+1}$ \\
$\cdot$ & $\cdot$ & $\cdot$ & $\cdot$ & $\cdot$ & $\cdot$ & $\cdot$ \\
$\cdot$ & $\cdot$ & $\cdot$ & $\cdot$ & $\cdot$ & $\cdot$ & $\cdot$ \\
$\cdot$ & $\cdot$ & $\cdot$ & $\cdot$ & $\cdot$ & $\cdot$ & $\cdot$ \\
$n$ & 2 & 1 & $Y_{1[2] n}$ & 1 & 2 & $Y_{2[1] n}$ \\
\hline
\end{tabular}

Table 2.1: Judgement post-stratification for a balanced, randomized two-sample design. Each row of the table gives a set. After reordering the sets, the first $n_{1}$ are Type 1 sets; the remaining $n-n_{1}$ are Type 2 sets. Notation for the responses includes as a subscript treatment, rank and set number.

\subsection{Models}

For a balanced, randomized two-sample design, data are collected for $2 n$ experimental units, among which $n$ units are from the treatment and the other $n$ are from the control.

\subsubsection{Location-Scale Models}

The following model, which is a slight extension of the additive model that underlies ANOVA, is used to describe the treatment and control responses and motivate our results:

$$
Y_{i j}=\mu_{i}+\sigma_{i} \epsilon_{i j} ; i=1,2, j=1,2, \ldots, n,
$$


where $\mu_{1}$ and $\mu_{2}$ are the control and treatment effects, $\sigma_{1}$ and $\sigma_{2}$ are the scale parameters of the control and treatment populations and $\epsilon_{i j} ; i=1,2, j=1,2, \ldots, n$, are i.i.d with mean zero and variance one.

In this model, the error term can be considered as a feature of each experimental unit and the experimental units will be ranked based on their error terms. A treatment is defined by a location and scale parameter, $\mu_{i}$ and $\sigma_{i}, i=1,2$, respectively, where 1 is reserved for control.

The randomization and ranking procedure creates $n$ sets of units. Let $[h]$ represent the rank assigned to a unit, and let $k$ represent the set. The ranking produces nonzero means for the random errors $\epsilon_{[h] k}$ and, with minimal assumptions, also yields a positive correlation between $\epsilon_{[h] k}$ and $\epsilon_{\left[h^{\prime}\right] k}$. This positive correlation is used in the ORR designs of Ozturk and MacEachern (2005). Model (2.1) can be modified to provide a basis for the analysis of the judgement ranked data as follows:

$$
Y_{i[h] k}=\mu_{i}+\sigma_{i} \epsilon_{[h] k}, i=1,2 ; h=1,2 ; k=1,2, \ldots, n
$$

where $Y_{i[h] k}$ is the response for the $i$-th treatment in the $k$-th pair with rank $h$, and $\epsilon_{[h] k}$ is the random error of the $k$-th set with $\operatorname{rank} h ; i=1,2, h=1,2$, and $k=1,2, \ldots, n$. The joint distribution of $\left(\epsilon_{[1] k}, \epsilon_{[2] k}\right)^{T}$ has mean $\left(\mu_{\epsilon[1]}, \mu_{\epsilon[2]}\right)^{T}$ and variance matrix

$$
\Sigma_{\epsilon}=\left[\begin{array}{cc}
\sigma_{\epsilon[1]}^{2} & \sigma_{\epsilon[1][2]} \\
\sigma_{\epsilon[1][2]} & \sigma_{\epsilon[2]}^{2}
\end{array}\right] .
$$

Let $\rho$ be the coefficient of correlation between the units within each set. Then $\sigma_{\epsilon[1][2]}$ is equivalent to $\rho \sigma_{\epsilon[1]} \sigma_{\epsilon[2]}$. Therefore, the joint distribution of responses for Type 1 sets, i.e. $\left(Y_{1[1] k}, Y_{2[2] k}\right)^{T}$, has mean $\left(\mu_{1}+\sigma_{1} \mu_{\epsilon[1]}, \mu_{2}+\sigma_{2} \mu_{\epsilon[2]}\right)^{T}$ and variance 
matrix

$$
\Sigma_{1}=\left[\begin{array}{cc}
\sigma_{1}^{2} \sigma_{\epsilon[1]}^{2} & \sigma_{1} \sigma_{2} \rho \sigma_{\epsilon[1]} \sigma_{\epsilon[2]} \\
\sigma_{1} \sigma_{2} \rho \sigma_{\epsilon[1]} \sigma_{\epsilon[2]} & \sigma_{2}^{2} \sigma_{\epsilon[2]}^{2}
\end{array}\right] .
$$

Similarly, the joint distribution of responses for the Type 2 sets, i.e. $\left(Y_{1[2] k}, Y_{2[1] k}\right)^{T}$, has mean $\left(\mu_{1}+\sigma_{1} \mu_{\epsilon[2]}, \mu_{2}+\sigma_{2} \mu_{\epsilon[1]}\right)^{T}$ and variance matrix

$$
\Sigma_{2}=\left[\begin{array}{cc}
\sigma_{1}^{2} \sigma_{\epsilon[2]}^{2} & \sigma_{1} \sigma_{2} \rho \sigma_{\epsilon[1]} \sigma_{\epsilon[2]} \\
\sigma_{1} \sigma_{2} \rho \sigma_{\epsilon[1]} \sigma_{\epsilon[2]} & \sigma_{2}^{2} \sigma_{\epsilon[1]}^{2}
\end{array}\right] .
$$

We now consider several models under different assumptions about the scale parameters. Our interests are in the changes to the variance matrix.

- Three-parameter model under the assumption of $\sigma_{1}^{2}=\sigma_{2}^{2}=\sigma^{2}$ and $\sigma_{\epsilon[1]}^{2}=\sigma_{\epsilon[2]}^{2}=$ $\sigma_{\epsilon}^{2}$. Parameters include $\sigma^{2}, \sigma_{\epsilon}^{2}$ and $\rho$. In this model, we have the same variance matrix for the two different type of sets, i.e.,

$$
\Sigma^{3}=\left[\begin{array}{cc}
\sigma^{2} \sigma_{\epsilon}^{2} & \sigma^{2} \rho \sigma_{\epsilon}^{2} \\
\sigma^{2} \rho \sigma_{\epsilon}^{2} & \sigma^{2} \sigma_{\epsilon}^{2}
\end{array}\right]
$$

The superscript 3 indicates that this is the variance matrix for the threeparameter model.

- Four-parameter models. There are two types of four parameter models leading to different variance matrices.

Case I: $\sigma_{1}^{2}=\sigma_{2}^{2}=\sigma^{2}$ and the parameters include $\sigma^{2}, \sigma_{\epsilon[1]}^{2}, \sigma_{\epsilon[2]}^{2}$ and $\rho$. Then the variance matrices for the two groups are

$$
\begin{aligned}
& \Sigma_{1}^{4 \sigma}=\left[\begin{array}{cc}
\sigma^{2} \sigma_{\epsilon[1]}^{2} & \sigma^{2} \rho \sigma_{\epsilon[1]} \sigma_{\epsilon[2]} \\
\sigma^{2} \rho \sigma_{\epsilon[1]} \sigma_{\epsilon[2]} & \sigma^{2} \sigma_{\epsilon[2]}^{2}
\end{array}\right], \\
& \Sigma_{2}^{4 \sigma}=\left[\begin{array}{cc}
\sigma^{2} \sigma_{\epsilon[2]}^{2} & \sigma^{2} \rho \sigma_{\epsilon[1]} \sigma_{\epsilon[2]} \\
\sigma^{2} \rho \sigma_{\epsilon[1]} \sigma_{\epsilon[2]} & \sigma^{2} \sigma_{\epsilon[1]}^{2}
\end{array}\right] .
\end{aligned}
$$


Although the above two matrices are not exactly the same, we notice that when we calculate the variance of differences between two responses within a set, this model would lead to equal variances for the two groups.

Case II: $\sigma_{\epsilon[1]}^{2}=\sigma_{\epsilon[2]}^{2}=\sigma_{\epsilon}^{2}$ and the parameters include $\sigma_{1}^{2}, \sigma_{2}^{2}, \sigma_{\epsilon}^{2}$ and $\rho$. Then we have the same variance matrices for the two types of sets

$$
\Sigma^{4 \sigma_{\epsilon}}=\left[\begin{array}{cc}
\sigma_{1}^{2} \sigma_{\epsilon}^{2} & \sigma_{1} \sigma_{2} \rho \sigma_{\epsilon}^{2} \\
\sigma_{1} \sigma_{2} \rho \sigma_{\epsilon}^{2} & \sigma_{2}^{2} \sigma_{\epsilon}^{2}
\end{array}\right]
$$

- Five-parameter model with parameters $\sigma_{1}^{2}, \sigma_{2}^{2}, \sigma_{\epsilon[1]}^{2}, \sigma_{\epsilon[2]}^{2}$, and $\rho$. Under this assumption, $\Sigma_{1}$ and $\Sigma_{2}$ are the variance matrices for the two groups. In this case, we have different variances for the two types of sets.

\subsubsection{Cell-Mean Models}

In a location-scale model, the response has the form of error term times the scale parameter plus its treatment (control) effect. The random error is considered as a feature of each experimental unit and the experimental units are ranked based on their errors. After the randomization and ranking procedure, Model (2.2) is used to analyze the judgement ranked data. We now consider a more general model by relaxing Model (2.1) as follows:

$$
Y_{i j}=\mu_{i}+\epsilon_{i j} ; i=1,2, j=1,2, \ldots, n
$$

where $\mu_{1}$ and $\mu_{2}$ are the control and treatment effects, and $\epsilon_{i j} ; i=1,2, j=1,2, \ldots, n$, are independent with mean zero and variance $\sigma_{i}^{2}$. In this model, the error term is still considered to be a feature of the EU and the EUs will be ranked based on their error terms. A treatment is defined by its cell mean, $\mu_{i}$. 
After applying the randomization and ranking procedure, we use the following model to analyze the judgement ranked data:

$$
Y_{i[h] k}=\mu_{i}+\epsilon_{i[h] k}, i=1,2 ; h=1,2 ; k=1,2, \ldots, n,
$$

where $Y_{i[h] k}$ is the response for the $i$-th treatment in the $k$-th set with rank $h$, and $\epsilon_{i[h] k}$ is the random error for the $i$-th treatment in the $k$-th set with rank $h ; i=1,2, h=1,2$, and $k=1,2, \ldots, n$. The joint distribution of $\left(\epsilon_{i[1] k}, \epsilon_{i^{\prime}[2] k}\right)^{T}$ has mean $\left(\mu_{i \in[1]}, \mu_{i^{\prime} \in[2]}\right)^{T}$ and variance matrix

$$
\Sigma_{\epsilon}=\left[\begin{array}{cc}
\sigma_{i \epsilon[1]}^{2} & \sigma_{i i^{\prime} \epsilon[1][2]} \\
\sigma_{i i^{\prime} \epsilon[1][2]} & \sigma_{i^{\prime} \epsilon[2]}^{2}
\end{array}\right] .
$$

The $n_{1}$ Type 1 sets are identically distributed as are the $n-n_{1}$ Type 2 sets, and all sets are mutually independent. Therefore, the two types of sets $\left(Y_{1[1] k}, Y_{2[2] k}\right)^{T}$ and $\left(Y_{1[2] k}, Y_{2[1] k}\right)^{T}$ have potentially different means and variance matrices with $E\left(Y_{i[h] k}\right)=$ $\mu_{i}+\mu_{i \epsilon[h]}=\mu_{i[h]}$.

\subsection{The Judgement Post-Stratification Estimator}

The SRS estimator of the contrast parameter is biased conditional on $n_{1}$ when $n_{1} \neq n / 2$. In this section, we propose a new estimator, $\hat{\Delta}_{1}$, which is unbiased for different values of $n_{1}$, has smaller variance than the SRS estimator, and is obtained from a generalized least squares estimator (GLS) of $\left[\mu_{1[1]}, \mu_{1[2]}, \mu_{2[2]}, \mu_{2[1]}\right]$ under Model (2.4).

To derive our new estimator, we assume Model (2.2) holds. Recall that the $R_{i} ; i=$ $1, \cdots, n$, indicate the ranking result. When the set $i$ belongs to Type $1, R_{i}=1,0$ otherwise. The following result is readily obtained.

$$
E\left(Y_{1[1] i}-Y_{2[2] i}\right)=E\left(Y_{1 i}-Y_{2 i} \mid R_{i}=1\right)=\mu_{1}+\sigma_{1} \mu_{\epsilon[1]}-\mu_{2}-\sigma_{2} \mu_{\epsilon[2]},
$$




$$
E\left(Y_{1[2] i}-Y_{2[1] i}\right)=E\left(Y_{1 i}-Y_{2 i} \mid R_{i}=0\right)=\mu_{1}+\sigma_{1} \mu_{\epsilon[2]}-\mu_{2}-\sigma_{2} \mu_{\epsilon[1]} .
$$

Under Model (2.4), we have

$$
\begin{aligned}
& E\left(Y_{1[1] i}-Y_{2[2] i}\right)=\mu_{1[1]}-\mu_{2[2]}, \\
& E\left(Y_{1[2] i}-Y_{2[1] i}\right)=\mu_{1[2]}-\mu_{2[1]} .
\end{aligned}
$$

Let $n_{1}$ be the number of sets in group 1 . Then $n_{1}=\sum_{i=1}^{n} R_{i}$. This number is a random variable distributed as $\operatorname{Bin}(n, 0.5)$. For a relatively large sample size, the probability of having no sets of one type, equivalently $n_{1}=0$ or $n$, is negligible. Estimation of the treatment contrast can be accomplished by first estimating the difference of two treatment means for the two different types respectively, and then combining these estimators. Assume the number of sets in each group is positive. Our new estimator can then be defined to be

$$
\hat{\Delta}_{1}=\frac{1}{2}\left[\frac{1}{n_{1}} \sum_{i=1}^{n_{1}}\left(Y_{1[1] i}-Y_{2[2] i}\right)+\frac{1}{n-n_{1}} \sum_{j=n_{1}+1}^{n}\left(Y_{1[2] j}-Y_{2[1] j}\right)\right] .
$$

The analysis of estimator (2.7) can proceed in two distinct directions. The unconditional view averages over the distribution of $n_{1}$ (whatever its realized value may be). The conditional view treats the observed value of $n_{1}$ as predetermined, and inference proceeds as if its value had been fixed by design. Note that $n_{1}$ is ancillary to the contrast parameter of interest. It contains no information about $\Delta$. In this work, we focus on conditional inference.

\subsubsection{Properties of JPS Estimator}

As discussed earlier, the ranking procedure guarantees that the ranks made after data collection are the same as if they were done before the experiment. Conditioning on the randomly formed sets and ranks, our data can fit into the general framework 
of URSS. Define $f(Y)$ and $f_{[r]}(Y)$ to be the pdf's of $Y$ and $Y_{[r]}$, where $Y_{[r]}$ is the $r$-th judgement order statistic. In a perfect ranking situation, $Y_{[r]}=Y_{(r)}$, where $Y_{(r)}$ is the $r$-th order statistic for $Y$. Define $\mu=E(Y), \mu_{[r]}=E\left[Y_{[r]}\right], \sigma^{2}=E\left[(Y-\mu)^{2}\right]$, $\sigma_{[r]}^{2}=E\left[\left(Y_{[r]}-\mu_{[r]}\right)^{2}\right]$, and $\tau_{[r]}=\mu_{[r]}-\mu$. Assume all means and variances are finite. Then we have the following results (Dell and Clutter, 1972)

$$
\begin{gathered}
f(Y)=\frac{1}{n} \sum_{r=1}^{n} f_{[r]}(Y), \\
\sum_{r=1}^{n} \mu_{[r]}=n \mu, \\
\sum_{r=1}^{n} \sigma_{[r]}^{2}=n \sigma^{2}-\sum_{r=1}^{n} \tau_{[r]}^{2} .
\end{gathered}
$$

In Models (2.1) and (2.2), let $f(\epsilon)$ and $f\left(\epsilon_{[h]}\right)$ be the pdf's of $\epsilon_{i j}$ and $\epsilon_{[h] k}$ respectively. It is easy to see that

$$
f(\epsilon)=\frac{1}{2} \sum_{h=1}^{2} f\left(\epsilon_{[h]}\right) .
$$

We can then obtain the following equations

$$
\begin{gathered}
\mu_{\epsilon[1]}=-\mu_{\epsilon[2]}, \\
\sigma_{\epsilon[1]}^{2}+\sigma_{\epsilon[2]}^{2}=2-\left(\mu_{\epsilon[1]}^{2}+\mu_{\epsilon[2]}^{2}\right) .
\end{gathered}
$$

For each pair, the ranking does not change the total variance of the error. Thus we have

$$
\sigma_{\epsilon[1]}^{2}+\sigma_{\epsilon[2]}^{2}=2-2 \rho \sigma_{\epsilon[1]} \sigma_{\epsilon[2]} .
$$

From (2.9) and (2.10), we derive the following relationship

$$
\rho \sigma_{\epsilon[1]} \sigma_{\epsilon[2]}=\frac{1}{2}\left(\mu_{\epsilon[1]}^{2}+\mu_{\epsilon[2]}^{2}\right) .
$$

This equation confirms the positive correlation between the units within each set. It also shows that this positive correlation highly depends on the ranking quality. For 
good ranking quality, we have $\left(\mu_{\epsilon[1]}^{2}+\mu_{\epsilon[2]}^{2}\right) \neq 0$. As ranking quality gets worse, the correlation gets smaller. Thus, under random ranking, $\mu_{\epsilon[1]}=\mu_{\epsilon[2]}=0$.

Under Models (2.3) and (2.4), we have

$$
E\left(Y_{i}\right)=E\left(Y_{i}, R_{i}=1\right)+E\left(Y_{i}, R_{i}=0\right)=\frac{1}{2}\left[E\left(Y_{i[1]}\right)+E\left(Y_{i[2]}\right)\right] ; i=1,2 .
$$

Hence, $\mu_{i}=\frac{1}{2}\left(\mu_{i[1]}+\mu_{i[2]}\right)$. The properties of $\hat{\Delta}_{1}$ can be summarized as the following theorems:

Theorem 1 (Unbiasedness and GLS) Assume the number of sets of each type is positive, i.e. $n_{1} \neq 0$ or $n$. Then, conditional on the specific pairing and ranking under Models (2.2) and (2.4), $\hat{\Delta}_{1}$ is unbiased for $\Delta$ and is obtained from the generalized least squares estimator of $\left[\mu_{1[1]}, \mu_{1[2]}, \mu_{2[2]}, \mu_{2[1]}\right]$.

Proof of Theorem 1: Under Model (2.2), in order to show that our new estimator $\hat{\Delta}^{1}$ is an unbiased estimator of the contrast parameter $\Delta=\mu_{1}-\mu_{2}$ conditional on the pairs and ranks, we need the relationship among parameters we derived before. According to (2.8), we have $\mu_{\epsilon[1]}=-\mu_{\epsilon[2]}$. Hence

$$
\begin{aligned}
E\left(\hat{\Delta}_{1}\right) & =\frac{1}{2}\left[E\left(\bar{y}_{1[1]}-\bar{y}_{2[2] .}\right)+E\left(\bar{y}_{1[2]}-\bar{y}_{2[1]} .\right)\right] \\
& =\frac{1}{2}\left[\left(\mu_{1}+\sigma_{1} \mu_{\epsilon_{[1]}}-\mu_{2}-\sigma_{2} \mu_{\epsilon_{[2]}}\right)+\left(\mu_{1}+\sigma_{1} \mu_{\epsilon_{[2]}}-\mu_{2}-\sigma_{2} \mu_{\epsilon_{[1]}}\right)\right] \\
& =\mu_{1}-\mu_{2} .
\end{aligned}
$$

Under Model (2.4), we have

$$
\begin{aligned}
E\left(\hat{\Delta}_{1}\right) & =\frac{1}{2}\left[E\left(\bar{y}_{1[1] \cdot}-\bar{y}_{2[2]}\right)+E\left(\bar{y}_{1[2] .}-\bar{y}_{2[1] .}\right)\right] \\
& =\frac{1}{2}\left[\left(\mu_{1[1]}-\mu_{2[2]}\right)+\left(\mu_{1[2]}-\mu_{2[1]}\right)\right] \\
& =\mu_{1}-\mu_{2} .
\end{aligned}
$$


To derive the generalized least squares estimator, we express our model using matrices as $Y=W \beta+\eta$, where $Y=\left[y_{1[1] 1}, \cdots, y_{1[1] n_{1}}, y_{1[2] n_{1}+1}, \cdots, y_{1[2] n}, y_{2[2] 1}, \cdots, y_{2[2] n_{1}}\right.$, $\left.y_{2[1] n_{1}+1}, \cdots, y_{2[1] n}\right]^{T}, \beta=\left[\mu_{1[1]}, \mu_{1[2]}, \mu_{2[2]}, \mu_{2[1]}\right]^{T}$, and $\eta=Y-W \beta$ represents a vector of ranked errors after subtracting their corresponding means. Note that the data are listed in the order of Table 2.1. The design matrix is thus,

$$
W=\left[\begin{array}{llll}
\mathbf{1}_{1 \times n_{1}} & \mathbf{0}_{1 \times\left(n-n_{1}\right)} & \mathbf{0}_{1 \times n_{1}} & \mathbf{0}_{1 \times\left(n-n_{1}\right)} \\
\mathbf{0}_{1 \times n_{1}} & \mathbf{1}_{1 \times\left(n-n_{1}\right)} & \mathbf{0}_{1 \times n_{1}} & \mathbf{0}_{1 \times\left(n-n_{1}\right)} \\
\mathbf{0}_{1 \times n_{1}} & \mathbf{0}_{1 \times\left(n-n_{1}\right)} & \mathbf{1}_{1 \times n_{1}} & \mathbf{0}_{1 \times\left(n-n_{1}\right)} \\
\mathbf{0}_{1 \times n_{1}} & \mathbf{0}_{1 \times\left(n-n_{1}\right)} & \mathbf{0}_{1 \times n_{1}} & \mathbf{1}_{1 \times\left(n-n_{1}\right)}
\end{array}\right]^{T} .
$$

The variance matrix $\Sigma$ of $\eta$ is a $2 n \times 2 n$ partitioned diagonal matrix.

Using the following fact, we get $\Sigma^{-1}$ :

$$
\left[\begin{array}{ll}
A_{11} & A_{12} \\
A_{21} & A_{22}
\end{array}\right]^{-1}=\left[\begin{array}{cc}
A_{11}^{-1}+A_{11}^{-1} A_{12} A_{2.1}^{-1} A_{21} A_{11}^{-1} & -A_{11}^{-1} A_{12} A_{2.1}^{-1} \\
-A_{2.1}^{-1} A_{21} A_{11}^{-1} & A_{2.1}^{-1}
\end{array}\right],
$$

where $A_{11}, A_{12}, A_{21}, A_{22}$ are all diagonal matrices. Linear algebra shows that the generalized least squares estimator of $\beta$ is

$$
\hat{\beta}=\left(W^{T} \Sigma^{-1} W\right)^{-1} W^{T} \Sigma^{-1} Y=\left(\bar{y}_{1[1] \cdot}, \bar{y}_{1[2]}, \bar{y}_{2[2]}, \bar{y}_{2[1]} .\right)^{T} .
$$

Therefore our new estimator $\hat{\Delta}_{1}$ is $a \hat{\beta}$, where $a=(1 / 2,1 / 2,-1 / 2,-1 / 2)$, and the variance is $a\left(W^{T} \Sigma^{-1} W\right)^{-1} a^{T}$.

\subsubsection{Comparison of The JPS and SRS Estimator}

The standard estimator of the difference of two sample means in the balanced, randomized two-sample design is the SRS estimator $\hat{\Delta}=\bar{Y}_{1}-\bar{Y}_{2}$. Our new estimator is based on a randomization and ranking procedure which is a combination of ORR and JPS. Inference using our new estimator is conditional on the randomly formed pairs and ranks. We wish to compare the performance of these two estimators both theoretically and numerically. 
Assume Models (2.1) and (2.2) hold. Then the variance for the SRS estimator becomes $\frac{1}{n}\left(\sigma_{1}^{2}+\sigma_{2}^{2}\right)$. The variance of the new estimator $\hat{\Delta}_{1}$ can be derived as follows:

$$
\begin{aligned}
& \operatorname{Var}\left(\hat{\Delta}_{1}\right) \\
& =\frac{1}{4}\left[\frac{1}{n_{1}^{2}} \operatorname{Var}\left(\sum_{i=1}^{n_{1}}\left(y_{1[1] i}-y_{2[2] i}\right)\right)+\frac{1}{\left(n-n_{1}\right)^{2}} \operatorname{Var}\left(\sum_{j=1}^{n-n_{1}}\left(y_{1[2] j}-y_{2[1] j}\right)\right)\right] \\
& =\frac{1}{4}\left[\frac{1}{n_{1}}\left(\sigma_{1}^{2} \sigma_{\epsilon[1]}^{2}+\sigma_{2}^{2} \sigma_{\epsilon[2]}^{2}-2 \sigma_{1} \sigma_{2} \sigma_{\epsilon[1][2]}\right)+\frac{1}{n-n_{1}}\left(\sigma_{1}^{2} \sigma_{\epsilon[2]}^{2}+\sigma_{2}^{2} \sigma_{\epsilon[1]}^{2}-2 \sigma_{1} \sigma_{2} \sigma_{\epsilon[1][2]}\right)\right] .
\end{aligned}
$$

Although comparisons of the variance of the SRS estimator with that of the JPS estimator seem complicated, the variances in above equation can be simplified under different models with certain assumptions.

- Three-parameter model under the assumption of $\sigma_{1}^{2}=\sigma_{2}^{2}=\sigma^{2}$ and $\sigma_{\epsilon[1]}^{2}=\sigma_{\epsilon[2]}^{2}=$ $\sigma_{\epsilon}^{2}$. Parameters include $\sigma^{2}, \sigma_{\epsilon}^{2}$ and $\rho$. Under this model, (2.10) and (2.11) can be reduced to

$$
\begin{gathered}
2 \sigma_{\epsilon}^{2}=2-2 \rho \sigma_{\epsilon}^{2} . \\
\rho \sigma_{\epsilon}^{2}=\frac{1}{2}\left(\mu_{\epsilon[1]}^{2}+\mu_{\epsilon[2]}^{2}\right) .
\end{gathered}
$$

Therefore, the variance of $\hat{\Delta}_{1}$ can be simplified as

$$
\begin{aligned}
\operatorname{Var}\left(\hat{\Delta}_{1}\right) & =\frac{1}{4}\left(\frac{1}{n_{1}}+\frac{1}{n-n_{1}}\right)\left(2 \sigma^{2} \sigma_{\epsilon}^{2}-2 \sigma^{2} \sigma_{\epsilon}^{2} \rho\right) \\
& =\frac{1}{4}\left(\frac{1}{n_{1}}+\frac{1}{n-n_{1}}\right)\left[2 \sigma^{2}\left(1-\left(\mu_{\epsilon[1]}^{2}+\mu_{\epsilon[2]}^{2}\right)\right)\right] .
\end{aligned}
$$

Here we notice that $\left(\mu_{\epsilon[1]}^{2}+\mu_{\epsilon[2]}^{2}\right) \leq 1$. Then we can obtain the relative efficiency of $\hat{\Delta}_{1}$ versus the SRS estimator as

$$
\begin{aligned}
R E_{1} & =\frac{\frac{1}{n} 2 \sigma^{2}}{\frac{1}{4}\left(\frac{1}{n_{1}}+\frac{1}{n-n_{1}}\right)\left(2 \sigma^{2}\left(1-\left(\mu_{\epsilon[1]}^{2}+\mu_{\epsilon[2]}^{2}\right)\right)\right)} \\
& =\frac{\frac{1}{n}}{\frac{1}{4}\left(\frac{1}{n_{1}}+\frac{1}{n-n_{1}}\right)}\left(1-\left(\mu_{\epsilon[1]}^{2}+\mu_{\epsilon[2]}^{2}\right)\right)^{-1} .
\end{aligned}
$$


The performance of the JPS estimator depends on the value of $n_{1}$. If $n_{1}=n / 2$, the variance of $\hat{\Delta}_{1}$ achieves its minimum. In this case, $R E_{1}=\left(1-\left(\mu_{\epsilon[1]}^{2}+\right.\right.$ $\left.\left.\mu_{\epsilon[2]}^{2}\right)\right)^{-1} \geq 1$ and the performance of the new estimator will only be affected by the strata means which are decided by the ranking quality. As the means of the random errors get larger, the relative efficiency increases. If we are in the situation of random ranking, we have $\mu_{\epsilon[1]}^{2}=\mu_{\epsilon[2]}^{2}=0$. Then the new estimator will perform as well as the sample mean estimator. Otherwise, it will deliver better performance than the standard estimator.

When $n_{1} \neq \frac{n}{2}$, we still have $R E_{1} \geq 1$ as long as $\frac{n}{2}-\frac{n}{2} \sqrt{\mu_{\epsilon[1]}^{2}+\mu_{\epsilon[2]}^{2}} \leq n_{1} \leq$ $\frac{n}{2}+\frac{n}{2} \sqrt{\mu_{\epsilon[1]}^{2}+\mu_{\epsilon[2]}^{2}}$. Notice that $n_{1}$ is distributed as $\operatorname{Bin}(n, 0.5)$, thus $n_{1} / n \rightarrow 1 / 2$ asymptotically. Then as $n \rightarrow \infty, \frac{\frac{1}{n}}{\frac{1}{4}\left(\frac{1}{n_{1}}+\frac{1}{n-n_{1}}\right)} \rightarrow 1$, and $R E_{1} \rightarrow\left(1-\left(\mu_{\epsilon[1]}^{2}+\right.\right.$ $\left.\left.\mu_{\epsilon[2]}^{2}\right)\right)^{-1} \geq 1$. Similar to the relationship for the judgement post-stratification and balanced ranked set sampling in a one-sample problem, the ratio of the conditional variance of the JPS estimator for contrast estimation to the variance of the ORR naive estimator with set size 2 tends to 1 almost surely.

- Four-parameter models. Case I: Assume $\sigma_{1}^{2}=\sigma_{2}^{2}=\sigma^{2}$. We have four parameters $\sigma^{2}, \sigma_{\epsilon[1]}^{2}, \sigma_{\epsilon[2]}^{2}$, and $\rho$ in the model. Then

$$
\begin{aligned}
R E_{2} & =\frac{\frac{1}{n} 2 \sigma^{2}}{\frac{1}{4}\left(\frac{1}{n_{1}}+\frac{1}{n-n_{1}}\right)\left(2 \sigma^{2}\left[1-2 \rho \sigma_{\epsilon[1]} \sigma_{\epsilon[2]}\right]\right)} \\
& =\frac{\frac{1}{n}}{\frac{1}{4}\left(\frac{1}{n_{1}}+\frac{1}{n-n_{1}}\right)}\left(1-\left(\mu_{\epsilon[1]}^{2}+\mu_{\epsilon[2]}^{2}\right)\right)^{-1} .
\end{aligned}
$$

Under this four-parameter model, we get the same variance as for the threeparameter model. Consequently the same argument as above applies. 
Case II: Alternatively, if we assume $\sigma_{\epsilon[1]}^{2}=\sigma_{\epsilon[2]}^{2}=\sigma_{\epsilon}^{2}$, then we have another four-parameter model with $\sigma_{1}^{2}, \sigma_{2}^{2}, \sigma_{\epsilon}^{2}$, and $\rho$ and

$$
R E_{3}=\frac{\frac{1}{n}}{\frac{1}{4}\left(\frac{1}{n_{1}}+\frac{1}{n-n_{1}}\right)}\left[1-\frac{1}{2}\left(1+\frac{2 \sigma_{1} \sigma_{2}}{\sigma_{1}^{2}+\sigma_{2}^{2}}\right)\left(\mu_{\epsilon[1]}^{2}+\mu_{\epsilon[2]}^{2}\right)\right]^{-1} .
$$

Thus $R E_{3} \rightarrow\left[1-\frac{1}{2}\left(1+\frac{2 \sigma_{1} \sigma_{2}}{\sigma_{1}^{2}+\sigma_{2}^{2}}\right)\left(\mu_{\epsilon[1]}^{2}+\mu_{\epsilon[2]}^{2}\right)\right]^{-1} \geq 1$ as $n \rightarrow \infty$.

- Five-parameter model. The variance in (2.12) is for the five-parameter model with $\sigma_{1}^{2}, \sigma_{2}^{2}, \sigma_{\epsilon[1]}^{2}, \sigma_{\epsilon[2]}^{2}$, and $\rho$. The relative efficiency of this model is the ratio of $\frac{1}{n}\left(\sigma_{1}^{2}+\sigma_{2}^{2}\right)$ and $(2.12)$. If we have $n_{1}=n / 2$, then

$$
R E_{4}=\left[1-\frac{1}{2}\left(1+\frac{2 \sigma_{1} \sigma_{2}}{\sigma_{1}^{2}+\sigma_{2}^{2}}\right)\left(\mu_{\epsilon[1]}^{2}+\mu_{\epsilon[2]}^{2}\right)\right]^{-1}
$$

It is worthwhile to point out that when $n_{1}=n / 2$, the variance of the judgement post-stratification estimator achieves its minimum under the three and fourparameter models. Under the five-parameter model, a balanced design may not deliver the best performance since the variances for the two groups are different. Neymann allocation might be a better choice in this case.

Under Models (2.3) and (2.4), the performance of $\hat{\Delta}_{1}$ also depends on the value of $n_{1}$. When $n_{1}=n / 2$, we have

$$
\operatorname{Var}\left(\hat{\Delta}_{1} \mid n_{1}=n / 2\right)=\frac{1}{2 n}\left[\left(\sigma_{1 \epsilon[1]}^{2}+\sigma_{2 \epsilon[2]}^{2}-2 \sigma_{12 \epsilon[2][1]}\right)+\left(\sigma_{1 \epsilon[2]}^{2}+\sigma_{2 \epsilon[1]}^{2}-2 \sigma_{21 \epsilon[1][2]}\right)\right]
$$

and

$$
\begin{aligned}
\operatorname{Var}(\hat{\Delta})= & E\left[\operatorname{Var}\left(\hat{\Delta} \mid n_{1}\right)\right]+\operatorname{Var}\left[E\left(\hat{\Delta} \mid n_{1}\right)\right] \\
= & \frac{1}{2 n}\left[\left(\sigma_{1 \epsilon[1]}^{2}+\sigma_{2 \epsilon[2]}^{2}-2 \sigma_{12 \epsilon[2][1]}\right)+\left(\sigma_{1 \epsilon[2]}^{2}+\sigma_{2 \epsilon[1]}^{2}-2 \sigma_{21 \epsilon[1][2]}\right)\right] \\
& +\frac{1}{4 n}\left[\left(\mu_{1[1]}-\mu_{2[2]}\right)-\left(\mu_{1[2]}-\mu_{2[1]}\right)\right]^{2} .
\end{aligned}
$$


Under Model (2.4), $\operatorname{Var}\left(\hat{\Delta}_{1} \mid n_{1}=n / 2\right) \leq \operatorname{Var}(\hat{\Delta})$, irrespective of the ranking procedure. Typically the inequality is strict. Since $n_{1}$ is distributed as $\operatorname{Bin}(\mathrm{n}, 1 / 2)$, we have $n_{1} / n \rightarrow 1 / 2$ as $n \rightarrow \infty$. Then, as $n \rightarrow \infty$, the relative efficiency of the JPS estimator versus the SRS estimator converges to a quantity bigger than one.

To compare the accuracy of the two estimators, we use mean squared error (MSE) since it incorporate both bias and variance. The simulation result is illustrated in Figure 2.1, from both conditional and unconditional viewpoints. In both cases, $\hat{\Delta}_{1}$ has smaller MSE than does $\hat{\Delta}$. We see that when $n_{1}$ assumes extreme values, $\hat{\Delta}$ has large MSE compared to $\hat{\Delta}_{1}$.

\subsubsection{Limiting Distribution and Inferential Procedures}

We will derive the limiting distribution of the new estimator and develop the hypothesis testing and interval estimation procedures conditioning on the sets and ranks observed.

Theorem 2 (Asymptotic Normality for $\hat{\Delta}_{1}$ ) Assume that Models (2.2) and (2.4) hold. As $\min \left(n_{1},\left(n-n_{1}\right)\right) \rightarrow \infty$, we have

$$
\sqrt{n}\left[\hat{\Delta}_{1}-\left(\mu_{1}-\mu_{2}\right)\right] \stackrel{D}{\rightarrow} N\left(0, \frac{1}{2}\left(\sigma_{X}^{2}+\sigma_{Z}^{2}\right)\right),
$$

or equivalently,

$$
\frac{\hat{\Delta}_{1}-\left(\mu_{1}-\mu_{2}\right)}{\sqrt{\frac{\sigma_{X}^{2}}{4 n_{1}}+\frac{\sigma_{Z}^{2}}{4\left(n-n_{1}\right)}}} \stackrel{D}{\rightarrow} N(0,1),
$$

where $\sigma_{X}^{2}$ and $\sigma_{Z}^{2}$ are the variances for the differences of responses in the two types of sets, i.e. $\sigma_{X}^{2}=\operatorname{Var}\left(Y_{1[1]}-Y_{2[2]}\right), \sigma_{Z}^{2}=\operatorname{Var}\left(Y_{1[2]}-Y_{2[1]}\right)$. This contrasts with

$$
\sqrt{n}\left[\hat{\Delta}-\left(\mu_{1}-\mu_{2}\right)\right] \stackrel{D}{\rightarrow} N\left(0, \frac{1}{2}\left(\sigma_{X}^{2}+\sigma_{Z}^{2}\right)+\frac{1}{4}\left(\mu_{X}-\mu_{Z}\right)^{2}\right),
$$

where $\mu_{X}=E\left(Y_{1[1]}-Y_{2[2]}\right)$ and $\mu_{Z}=E\left(Y_{1[2]}-Y_{2[1]}\right)$. 
comparison of the mean square errors of delta and delta1 for sample size 10

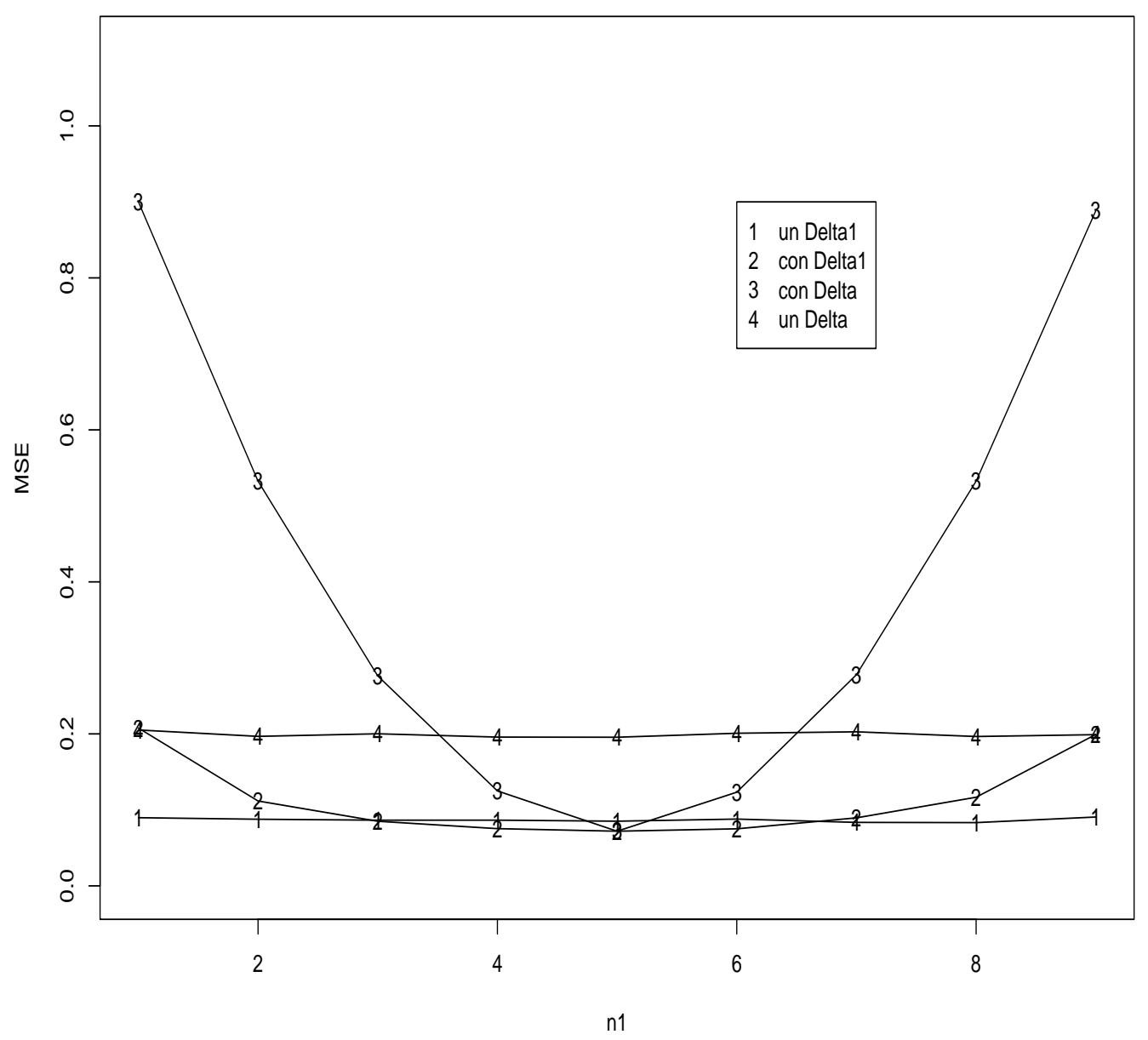

Figure 2.1: Comparison of the mean square errors both from unconditional and conditional point of view for $\hat{\Delta}_{1}$ with symbols 1 and 2 , and $\hat{\Delta}$ with symbols 3 and 4 . The sample size is 10 and ranking is perfect. 
These good properties of the JPS estimator become the basis for the corresponding inferential procedures.

Proof of Theorem 2: To simplify the illustration, we first define some new notation for our data. Let $X_{i} ; i=1,2, \cdots, n_{1}$, denote the differences of the responses in the first group where the control experimental units are assigned rank 1, i.e., $X_{i}=$ $Y_{1[1] i}-Y_{2[2] i} ; i=1,2, \cdots, n_{1}$. Similarly, for the other group, $Z_{j}=Y_{1[2] j}-Y_{2[1] j}$; $j=n_{1}+1, \cdots, n$. Then $X_{1}, X_{2}, \cdots, X_{n_{1}}$ are i.i.d. random variables with distribution $F_{X}$, and $Z_{n_{1}+1}, Z_{n_{1}+2}, \cdots, Z_{n}$ are also i.i.d. with distribution $G_{Z}$. The $X^{\prime} s$ and $Z^{\prime} s$ are independent, conditional on the observed $n_{1}$. Let $\mu_{X}$ and $\sigma_{X}^{2}$ be the mean and variance for $X_{i}$, and $\mu_{Z}$ and $\sigma_{Z}^{2}$ be the mean and variance for $Z_{j}$, respectively. Assume that both variances are finite.

As stated earlier, $n_{1}$ is distributed as $\operatorname{Bin}(n, 1 / 2)$. Let $(\Omega, \mathcal{F}, \mathcal{P})$ denote the probability space. By Borel's Strong Law of Large Numbers (SLLN) for Bernoulli random variables (Lehmann 1998), we have that $\frac{n_{1}}{n} \rightarrow 1 / 2$ w.p.1. We define a set $N \in \mathcal{F}$ for which $P(N)=0, \frac{n_{1}}{n} \rightarrow 1 / 2$ for all $\omega \notin N$. Equivalently, let $\Omega_{c}$ be the set of probability one for which we have convergence. $\Omega=\Omega_{c} \bigcup N$. Partition $\Omega_{c}$ into a countable collection of subsets, $\left\{\Omega_{c_{i}}\right\}_{i=1}^{\infty}$, upon each of which the sequence $\left\{n_{1}\right\}_{n=1}^{\infty}$ is deterministic, and where the sequences of $n_{1}$ differ for distinct $\Omega_{c_{i}}$. Then apply the standard Central Limit Theorem for i.i.d. random variables to $\Omega_{c_{i}}$. For this set, $\min \left(n_{1},\left(n-n_{1}\right)\right) \rightarrow \infty$, and so we have $\left(\sqrt{n_{1}}\left(\bar{X}-\mu_{X}\right), \sqrt{n-n_{1}}\left(\bar{Z}-\mu_{Z}\right)\right) \stackrel{D}{\rightarrow}\left(X^{*}, Z^{*}\right)$, where $X^{*}, Z^{*}$ are independent random variables with $X^{*} \sim N\left(0, \sigma_{X}^{2}\right)$ and $Z^{*} \sim N\left(0, \sigma_{Z}^{2}\right)$. By Slutsky's Theorem, applied to the function $f\left(X^{*}, Z^{*}\right)=\left(X^{*}+Z^{*}\right) / 2$, we have

$$
\sqrt{n}\left[\hat{\Delta}_{1}-\left(\mu_{1}-\mu_{2}\right)\right] \stackrel{D}{\rightarrow} N\left(0, \frac{1}{2}\left(\sigma_{X}^{2}+\sigma_{Z}^{2}\right)\right)
$$


and

$$
\frac{\hat{\Delta}_{1}-\left(\mu_{1}-\mu_{2}\right)}{\sqrt{\frac{\sigma_{X}^{2}}{4 n_{1}}+\frac{\sigma_{Z}^{2}}{4\left(n-n_{1}\right)}}} \stackrel{D}{\rightarrow} N(0,1) .
$$

Since

$$
\begin{aligned}
\operatorname{Var}(\hat{\Delta}) & =E\left[\operatorname{Var}\left(\hat{\Delta} \mid n_{1}\right)\right]+\operatorname{Var}\left[E\left(\hat{\Delta} \mid n_{1}\right)\right] \\
& =E\left[\frac{n_{1} \sigma_{X}^{2}+\left(n-n_{1}\right) \sigma_{Z}^{2}}{n^{2}}\right]+\operatorname{Var}\left[\frac{n_{1}}{n} \mu_{X}+\frac{n-n_{1}}{n} \mu_{Z}\right] \\
& =\frac{1}{2 n}\left(\sigma_{X}^{2}+\sigma_{Z}^{2}\right)+\frac{1}{4 n}\left(\mu_{X}-\mu_{Z}\right)^{2}
\end{aligned}
$$

we have

$$
\sqrt{n}\left[\hat{\Delta}-\left(\mu_{1}-\mu_{2}\right)\right] \stackrel{D}{\rightarrow} N\left(0, \frac{1}{2}\left(\sigma_{X}^{2}+\sigma_{Z}^{2}\right)+\frac{1}{4}\left(\mu_{X}-\mu_{Z}\right)^{2}\right) .
$$

A large sample testing procedure can then be obtained from the above asymptotic property of $\hat{\Delta}_{1}$. To test the hypothesis $H_{0}: \mu_{1}-\mu_{2}=0$ against $H_{a}: \mu_{1}-\mu_{2} \neq 0$, we define

$$
Z_{n}=\frac{\hat{\Delta}_{1}}{\sqrt{\frac{\sigma_{x}^{2}}{4 n_{1}}+\frac{\sigma_{z}^{2}}{4\left(n-n_{1}\right)}}} .
$$

Under the null hypothesis, $Z_{n}$ is asymptotically standard normal. If $\sigma_{X}^{2}$ and $\sigma_{Z}^{2}$ both are known, and $u_{\alpha}$ is the $\alpha$-th quantile of the standard normal distribution, then it follows that the rejection region

$$
\left|Z_{n}\right|=\frac{\left|\hat{\Delta}_{1}\right|}{\sqrt{\frac{\sigma_{X}^{2}}{4 n_{1}}+\frac{\sigma_{Z}^{2}}{4\left(n-n_{1}\right)}}} \geq u_{1-\alpha / 2}
$$

provides a test of $H_{0}$ against $H_{a}$ at asymptotic level $\alpha$.

We consider two cases when the parameters $\sigma_{X}^{2}$ and $\sigma_{Z}^{2}$ are unknown:

- Assume $\sigma_{X}^{2}=\sigma_{Z}^{2}=\sigma_{p}^{2}$. Under Model (2.2), this equal variance assumption is satisfied when we have the three or four-parameter models. Equivalently if we assume that we have at least one assumption of $\sigma_{1}^{2}=\sigma_{2}^{2}$ and $\sigma_{\epsilon[1]}^{2}=\sigma_{\epsilon[2]}^{2}$ holding, 
we have equal variances for $X$ and $Z$. In this case, there are two independent estimators for $\sigma_{p}^{2}$,

$$
S_{X}^{2}=\frac{1}{n_{1}-1} \sum_{j=1}^{n_{1}}\left(X_{j}-\bar{X}\right)^{2}
$$

and

$$
S_{Z}^{2}=\frac{1}{n-n_{1}-1} \sum_{j=1}^{n-n_{1}}\left(Z_{j}-\bar{Z}\right)^{2},
$$

where $\bar{X}=\frac{1}{n_{1}} \sum_{i=1}^{n_{1}} X_{i}, \bar{Z}=\frac{1}{n-n_{1}} \sum_{j=1}^{n-n_{1}} Z_{j}$. Then the pooled estimator for $\sigma_{p}^{2}$ can be given as

$$
S_{p}^{2}=\frac{1}{n-2}\left[\left(n_{1}-1\right) S_{X}^{2}+\left(n-n_{1}-1\right) S_{Z}^{2}\right]
$$

It can be easily shown that $S_{p}^{2}$ is a consistent estimator of $\sigma_{p}^{2}$. Define

$$
T_{n}=\frac{\hat{\Delta}_{1}}{\sqrt{\frac{1}{4}\left(\frac{1}{n_{1}}+\frac{1}{\left(n-n_{1}\right)}\right) S_{p}^{2}}} .
$$

Following Slutsky's theorem, we can establish that $T_{n}$ converges to a standard normal distribution under the null hypothesis. Hence the test with rejection region

$$
\left|T_{n}\right|=\frac{\left|\hat{\Delta}_{1}\right|}{\sqrt{\frac{1}{4}\left(\frac{1}{n_{1}}+\frac{1}{\left(n-n_{1}\right)}\right) S_{p}^{2}}} \geq u_{1-\alpha / 2}
$$

also has an asymptotic level $\alpha$. We note that $T_{n}$ is the two-sample $t$-test statistic with $n-2$ degrees of freedom (d.f.). The simulation study of the next section shows that the $t$ reference distribution provides a test which holds its level better than a test based on a $z$ reference distribution.

- If $\sigma_{X}^{2} \neq \sigma_{Z}^{2}$, we are under the five-parameter model. In this case, we propose replacing $\sigma_{X}^{2}$ with $S_{X}^{2}$ and $\sigma_{Z}^{2}$ with $S_{Z}^{2}$ and using the $t$-distribution with d.f. obtained by Satterthwaite's approximation as the reference distribution. We 
can show under the null hypothesis that

$$
\frac{\hat{\Delta}_{1}}{\sqrt{\frac{S_{X}^{2}}{4 n_{1}}+\frac{S_{Z}^{2}}{4\left(n-n_{1}\right)}}} \rightarrow N(0,1)
$$

To see this, we write the left side of (2.14) as

$$
\frac{\hat{\Delta}_{1}}{\sqrt{\frac{\sigma_{X}^{2}}{4 n_{1}}+\frac{\sigma_{Z}^{2}}{4\left(n-n_{1}\right)}}} \frac{\sqrt{\frac{\sigma_{X}^{2}}{4 n_{1}}+\frac{\sigma_{Z}^{2}}{4\left(n-n_{1}\right)}}}{\sqrt{\frac{S_{X}^{2}}{4 n_{1}}+\frac{S_{Z}^{2}}{4\left(n-n_{1}\right)}}} .
$$

Now $\frac{\sqrt{n} \sqrt{\frac{\sigma_{X}^{2}}{4 n_{1}}+\frac{\sigma_{Z}^{2}}{4\left(n-n_{1}\right)}}}{\sqrt{\frac{\sigma_{X}^{2}}{4 \lambda}+\frac{\sigma_{Z}^{2}}{4(1-\lambda)}}} \rightarrow 1$, and $\frac{\sqrt{n} \sqrt{\frac{S_{X}^{2}}{4 n_{1}}+\frac{S_{Z}^{2}}{4\left(n-n_{1}\right)}}}{\sqrt{\frac{S_{X}^{2}}{4 \lambda}+\frac{S_{Z}^{2}}{4(1-\lambda)}}} \rightarrow 1$. Hence by Slutsky's theorem, (2.14) is readily obtained. Therefore, the test with rejection region

$$
\left|T_{n}^{*}\right|=\frac{\left|\hat{\Delta}_{1}\right|}{\sqrt{\frac{S_{X}^{2}}{4 n_{1}}+\frac{S_{Z}^{2}}{4\left(n-n_{1}\right)}}} \geq u_{1-\alpha / 2}
$$

also has asymptotic level $\alpha$. Similar to the equal variance situation, for small sample sizes, Student's $t$-distribution provides a better approximation than normal distribution.

Interval estimation for the contrast parameter is obtained by inverting the acceptance region of the hypothesis test. Let $t_{v}^{\alpha}$ be the $\alpha$-th quartile of the student's $t$ distribution with $v$ d.f. Then under the equal variance assumption a $(1-\gamma) 100 \%$ asymptotic confidence interval for $\mu_{1}-\mu_{2}$ is given by

$$
\hat{\Delta}_{1} \pm u_{1-\gamma / 2} \sqrt{\frac{1}{4}\left(\frac{1}{n_{1}}+\frac{1}{\left(n-n_{1}\right)}\right) S_{p}^{2}}
$$

or

$$
\hat{\Delta}_{1} \pm t_{n-2}^{1-\gamma / 2} \sqrt{\frac{1}{4}\left(\frac{1}{n_{1}}+\frac{1}{\left(n-n_{1}\right)}\right) S_{p}^{2}} .
$$

Similarly, for the unequal variance assumption, we have

$$
\hat{\Delta}_{1} \pm u_{1-\gamma / 2} \sqrt{\frac{S_{X}^{2}}{4 n_{1}}+\frac{S_{Z}^{2}}{4\left(n-n_{1}\right)}},
$$


or

$$
\hat{\Delta}_{1} \pm t_{\hat{v}}^{1-\gamma / 2} \sqrt{\frac{S_{X}^{2}}{4 n_{1}}+\frac{S_{Z}^{2}}{4\left(n-n_{1}\right)}}
$$

where

$$
\hat{v}=\frac{\left(\frac{S_{X}^{2}}{n_{1}}+\frac{S_{Z}^{2}}{n-n_{1}}\right)^{2}}{\frac{S_{X}^{4}}{n_{1}^{2}\left(n_{1}-1\right)}+\frac{S_{Z}^{4}}{\left(n-n_{1}\right)^{2}\left(n-n_{1}-1\right)}} .
$$

One-sided tests and the corresponding confidence limits then follow.

\subsection{Simulations}

We investigate the performance of the JPS estimator $\hat{\Delta}_{1}$ versus the SRS estimator $\hat{\Delta}$ via a simulation study based on Models (2.1) and (2.2). The study includes three parts with the goal of showing superiority of the proposed estimator and corresponding test procedures over its competitor. The first part studies the properties of the JPS estimator $\hat{\Delta}_{1}$ and the SRS estimator $\hat{\Delta}$ and compares the mean square errors of estimation for different sample sizes with various ranking quality. The second part of the simulation study investigates the performance of all proposed test procedures through consideration of level and power. The third part examines the robustness of the inference associated with the new estimators to violations of Model (2.1).

Dell and Clutter (1972) proposed a model to describe rankings that range from perfect to random. Under this model, units are ranked based on estimates of the true error terms. Each estimate includes the true error plus random noise, i.e., $u_{i}=\epsilon_{i}+w_{i}$, where $\left(\epsilon_{i}, w_{i}\right) ; i=1,2, \cdots, n$, are all mutually independent, $\epsilon_{i}$ is normal with mean 0 and variance 1 and the random noise $w_{i}$ is normal with mean 0 and variance $\tau^{2}$. Thus $\tau^{2}$, the variance of $w$, controls the content of ranking information. Equivalently, the quality of rankings can be described by $\rho=1 / \sqrt{\left(1+\tau^{2}\right)}$. In studies of RSS, the 
rankings provide little benefit when $\rho \leq 0.5$, moderate benefit when $0.5<\rho \leq 0.7$, and substantial benefit when $\rho \geq 0.9$.

The data for our simulation study are generated and ranked by the randomization and ranking procedures described in previous section. The steps are as follows: (1). Generate $\epsilon_{i} ; i=1,2, \cdots, 2 n$, i.i.d. from $N(0,1)$ and $w_{i} ; i=1, \cdots, 2 n$, i.i.d. from $N\left(0, \tau^{2}\right)$. Then calculate $u_{i}=w_{i}+\epsilon_{i} ; i=1, \cdots, 2 n$. (2). Randomly assign $n$ of the $\epsilon_{i}$ to the treatment group and the other $n$ to the control group. Therefore, we have $\epsilon_{i j} ; i=1,2, j=1, \cdots, n$ and the response $Y_{i j}=\mu_{i}+\sigma_{i} \epsilon_{i j}$. (3). Rank the randomly formed $n$ pairs of units using $u_{i}$, the estimates of $\epsilon_{i}$, and rearrange the data. Then $Y_{i[h] k}=\mu_{i}+\sigma_{i} \epsilon_{[h] k} ; i=1,2 ; h=1,2 ; k=1, \cdots, n_{i}$. (4). Finally compute $n_{1}$, the number of Type 1 sets, the new estimator $\hat{\Delta}_{1}$, and the SRS estimator $\hat{\Delta}$.

The properties of the two estimators are investigated graphically through a simulation study based on 10,000 replicates of the 4-step process given above. The true difference of treatment effects $\Delta=\mu_{1}-\mu_{2}$ is taken to be -1 . All scale parameters are set to be 1 . Figure 2.2 shows the normal probability plots for the new estimator $\hat{\Delta}_{1}$ and the SRS estimator $\hat{\Delta}$ with sample size equal to 10 under the perfect ranking situation. Compared to $\hat{\Delta}$, the new estimator $\hat{\Delta}_{1}$ has heavy tails on both sides and a slightly flat slope. The several outliers on both sides correspond to the small or large values for $n_{1}$ and the flat slope indicates less variation compared to the SRS estimator $\hat{\Delta}$. Figures 2.3-2.5 are the normal probability plots for the two estimators for different values of $n_{1}$. We can see that when $n_{1}$ takes middle range values, e.g., $n_{1}=3,4,5,6,7$, the two estimators are quite similar, while for extreme values like $n_{1}=1,2,8,9$, the new estimator becomes skewed. 
Figures 2.6-2.9 are the box plots for $\hat{\Delta}_{1}$ and $\hat{\Delta}$ under different ranking situations. The box plots in the lower right panel of Figures 2.6-2.9 are for the unconditional distribution of the two estimators. The distribution conditional on the observed values of $n_{1}$ as $2,4,5,7,9$ are also plotted. We can see that both estimators are unbiased estimators of $\Delta$ from an unconditional point of view and they are equivalent when $n_{1}=n / 2$. As $n_{1}$ gets small, $\hat{\Delta}$ becomes biased conditional on $n_{1}$ and tends to underestimate the parameter. When $n_{1}$ gets larger than $n / 2, \hat{\Delta}$ tends to overestimate the true difference between $\mu_{1}$ and $\mu_{2}$. Moreover, as the ranking quality decreases, the number of Type 1 sets, $n_{1}$, contains less and less information about the EUs. Therefore, the advantage of $\hat{\Delta}_{1}$ over $\hat{\Delta}$ in terms of bias becomes smaller and smaller.

Table 2.2 compares the mean square errors of $\hat{\Delta}_{1}$ and $\hat{\Delta}$ with sample sizes varying from 10 to 100 . For the purpose of illustration, we only list the mean square errors (MSE) and the estimated relative efficiency (RE) for both estimators for selected sample sizes. The superiority of our new estimator is well-demonstrated by this table. In the perfect ranking situation, the JPS estimator has significant improvement over the SRS estimator. As ranking quality decreases, the performance of $\hat{\Delta}_{1}$ decreases. When the ranking becomes random, $\hat{\Delta}_{1}$ perform poorly when $n$ is small and it gets close to $\hat{\Delta}$ as $n$ becomes large. This matches well with the analysis of relative efficiency previously, since in the random ranking situation, the new estimator will perform as well as the SRS estimator only when $n_{1}=n / 2$. When the sample size is small, there is a big chance for $n_{1}$ to be close to or equal to 0 or $n$. As $n$ increases, the probability of $n_{1}=n / 2$ becomes relatively large.

In the second part of the simulation study, the performance of all proposed test procedures of our new estimator $\hat{\Delta}_{1}$ as well as the SRS estimator $\hat{\Delta}$ in a two sample 
$t$-test are investigated. Let $T_{n}^{S R S}=\frac{\bar{y}_{1 \cdot}-\bar{y}_{2 .}}{\sqrt{\frac{2}{n} S_{p S R S}^{2}}}$, where $S_{p S R S}^{2}=\frac{1}{2 n-2}\left(\sum_{j=1}^{n}\left(y_{1 j}-y_{1} \cdot\right)^{2}+\right.$ $\left.\sum_{j=1}^{n}\left(y_{2 j}-y_{2}\right)^{2}\right)$, denote the pooled two sample $t$-test. We estimate the levels of a nominal $\alpha=0.05$ test for all the test statistics and compare their empirical powers.

Table 2.3 reports the estimated levels of a nominal $\alpha=0.05$ test based on 10,000 replicates. We compare four types of testing procedures, three of which correspond to the student's $t$-distribution $\left(T_{n}\right)$ with $n-2$ d.f., the limiting normal distribution $\left(Z_{n}\right)$, and Satterthwaite's approximation $\left(S_{n}\right)$ for the JPS estimator. The fourth test procedure is for the SRS estimator, i.e., student's $t$-distribution $\left(T_{n}^{S R S}\right)$ with $2 n-2$ d.f. All scale parameters are set to 1 and the hypothesis test is $H_{0}: \mu_{1}=\mu_{2}$ versus $H_{a}: \mu_{1} \neq \mu_{2}$. We can easily see that except for the limiting normal distribution, all the other three approximations provide reasonably good estimates for the type I error. For small sample sizes, the student's $t$-distribution with $n-2$ d.f. is the most conservative test among all the testing procedures of $\hat{\Delta}_{1}$. In contrast to the t-distribution, the normal approximation requires a large sample size for nominal and actual levels to be close.

The estimated levels of tests based on $\hat{\Delta}_{1}$, conditioning on different values of $n_{1}$, are calculated for sample sizes of 10, 20, and 100 in Tables 2.4-2.6. By the property of symmetry of the estimator, we calculate the level based on the weighted average of the level of $n_{1}$ and $n-n_{1}$. For sample size equals to 10 and $n_{1}=1,9$, the variances of the limiting normal and Satterthwaite's estimation are underestimated leading to large rejection proportions. Hence the results are marked by NA. For the sample size $n=100$, NA is also used to indicate empty cells.

Figure 2.10 shows plots of the estimated levels for $T_{n}$ when the nominal level is $\alpha=0.05$ under the $t$-distribution with $n-2$ d.f. and for $T_{n}^{S R S}$ under the $t$-distribution 
with $2 n-2$ d.f. We can see that $T_{n}$ holds its level across the range of values for $n_{1}$. In contrast, $T_{n}^{S R S}$ has a level that is often far from nominal - high for extreme $n_{1}$ and low for middling $n_{1}$. These differences become smaller as the ranking quality decreases.

We also calculated empirical powers both for $T_{n}$ and $T_{n}^{S R S}$. Figures 2.11-2.14 present the results of our simulation study based on 10,000 replicates with the location shift parameter $\Delta=0.1(0.1) 2$ under different ranking qualities, where $0.1(0.1) 2=$ $(0.1,0.2, \ldots, 2)$. It is clear that the test $T_{n}$ based on $\hat{\Delta}_{1}$ outperforms the two sample $t$-test for the SRS estimator $T_{n}^{S R S}$ under a good ranking quality ranging from $\rho=1$ to 0.5. Similarly, the difference between the two test statistics gets smaller as sample size becomes larger and the ranking quality decreases.

The third part of the simulation study focuses on the robustness of $\hat{\Delta}_{1}$. Table 2.7 presents the estimated levels for a nominal level $\alpha=0.05$ test based on 10,000 replicates when the control and treatment variances are set to be 1 and 4 respectively. We compare four test statistics including $T_{n}$ and $Z_{n}$ for $\hat{\Delta}_{1}, T_{n}^{S R S}$, and $Z_{n}^{S R S}$ for $\hat{\Delta}$. We find that the levels for the pooled $t$-test of $\hat{\Delta}$ become large compared to the equal variance assumption, while the $t$-test for $\hat{\Delta}_{1}$ shows consistent performance. The normal approximation for both estimators requires larger sample sizes to maintain an $\alpha=0.05$ level.

We then compare the empirical powers for the above four test statistics. Figure 2.15 illustrates the results under the perfect ranking situation based on 10,000 replicates. The variances for the two treatments are still set to be 1 and 4 . Both the pooled $t$ and limiting normal distributions for $\hat{\Delta}_{1}$ outperform that for $\hat{\Delta}$. For each 
estimator, the test statistics based on the normal approximation has higher power than that for the pooled $t$-test.

Table 2.8 shows the performance of the estimators when the underlying distribution of the error term $\epsilon$ is actually skewed under the perfect ranking situation. Here we use the lognormal as the true distribution and the shape parameter $\sigma$ to control the skewness. As the value of $\sigma$ gets larger, the lognormal distribution becomes more skewed. In this study, the mean of $\log (\epsilon)$ is 0 and standard deviations of $\log (\epsilon)$ are set to $0.5,1,2$ and 4 . It is well demonstrated that when the skewness increases all test levels become smaller. For relatively small sample sizes the test level for our new estimator decreases more slowly than that for the SRS estimator. This indicates that the asymptotic theory may not work well if the underlying distribution is very skewed. In practice, it is advisable to perform certain transformations on the data before applying the asymptotic tests if the data appear to be very skewed.

Table 2.9 gives the estimated levels when the underlying distribution has a thick tail under perfect ranking. Here we use the $t$-distribution and the d.f.'s are chosen to be $5,10,20$, and 40 to control the thickness of the tail. The levels are only slightly smaller for thicker tails and there are no significant differences in the estimated levels from the case when the normal distribution is used. 

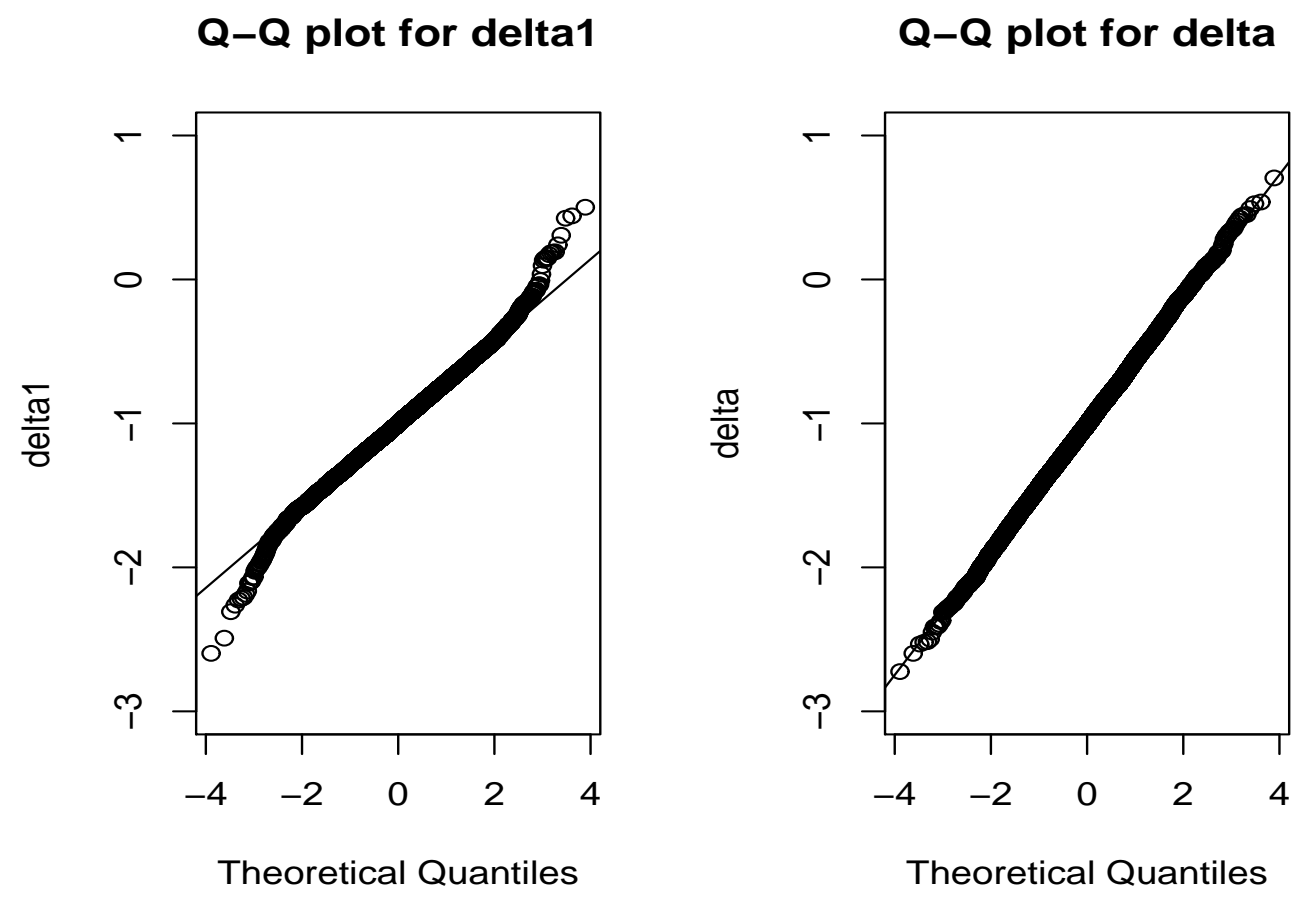

Figure 2.2: Normal Probability plots of $\hat{\Delta}_{1}$ and $\hat{\Delta}$ for perfect ranking when $n=10$ 


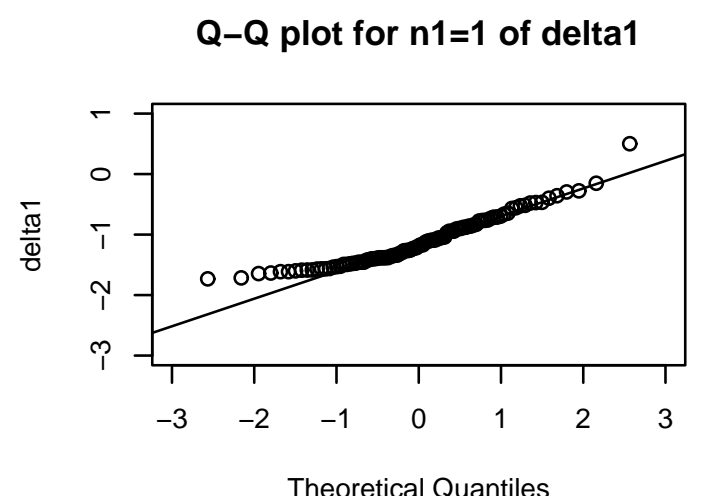

Theoretical Quantiles
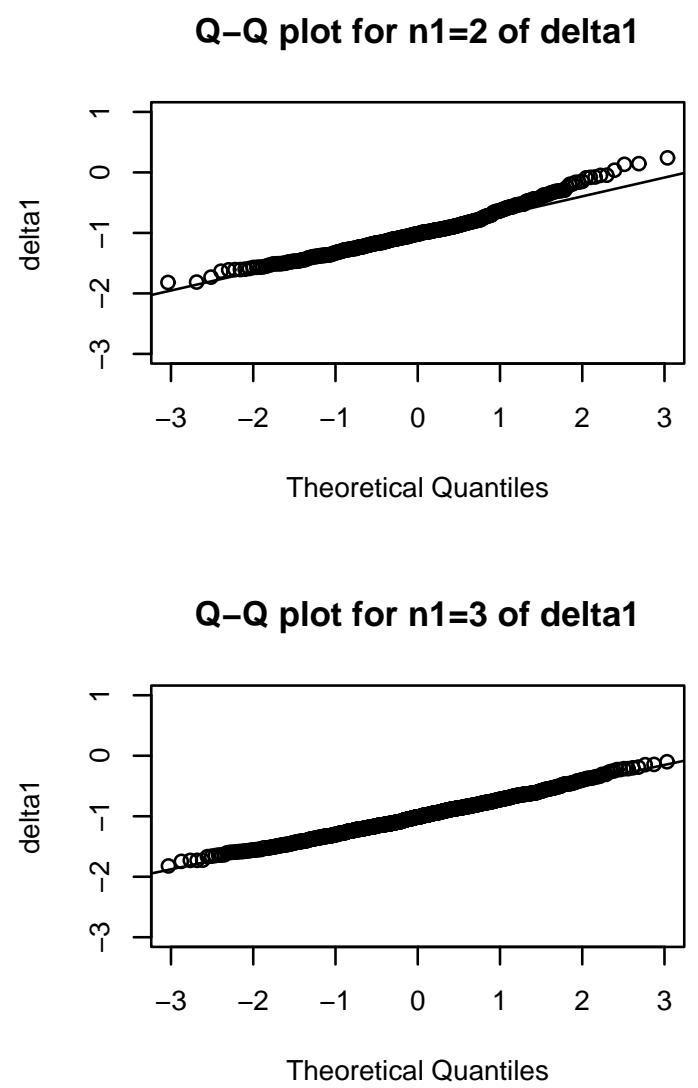
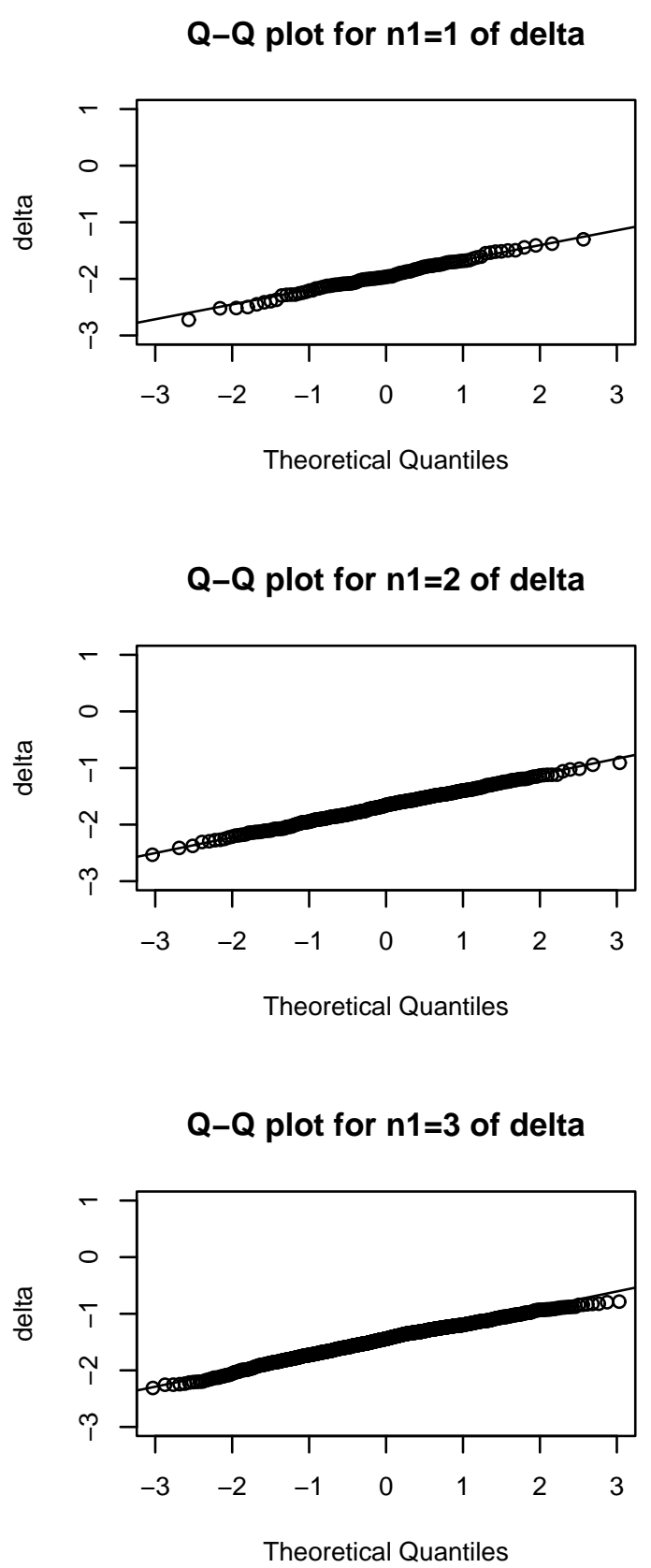

Figure 2.3: Normal Probability plots of $\hat{\Delta}_{1}$ and $\hat{\Delta}$ for perfect ranking when $n=10$ and $n_{1}=1,2,3$ 


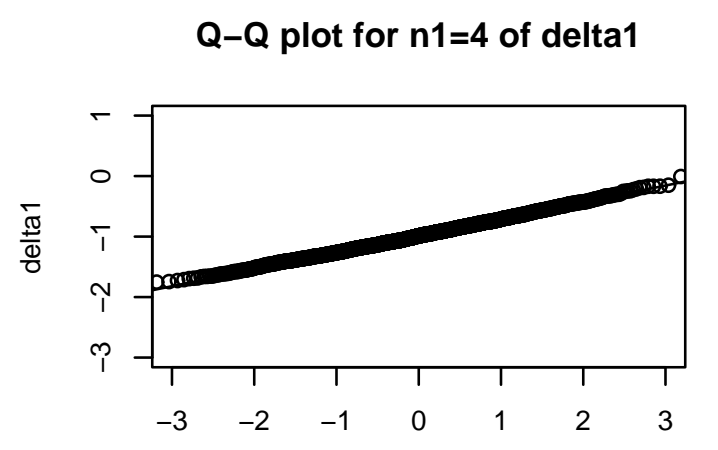

Theoretical Quantiles
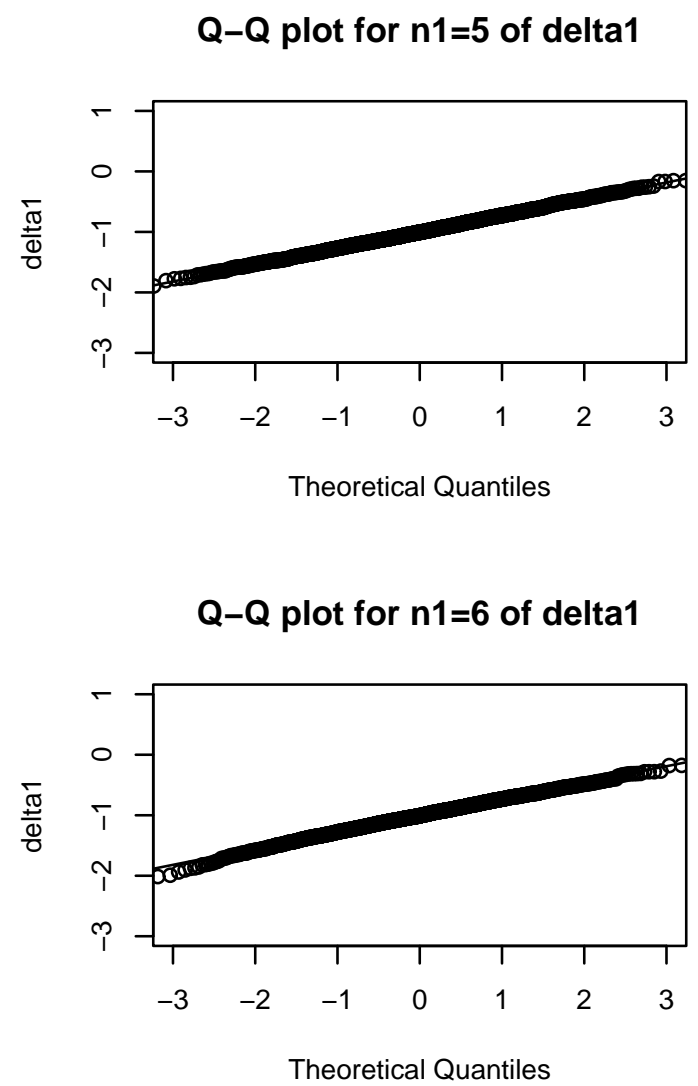
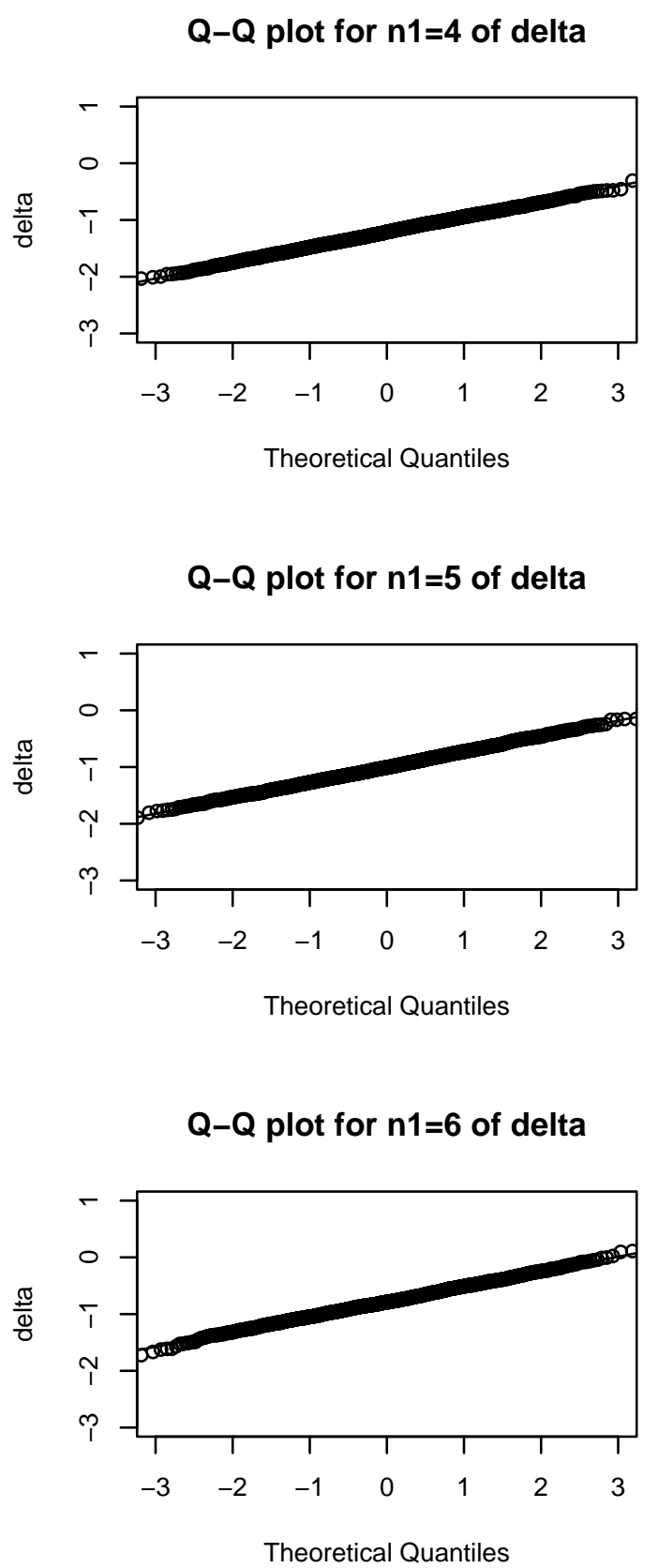

Figure 2.4: Normal Probability plots of $\hat{\Delta}_{1}$ and $\hat{\Delta}$ for perfect ranking when $n=10$ and $n_{1}=4,5,6$ 


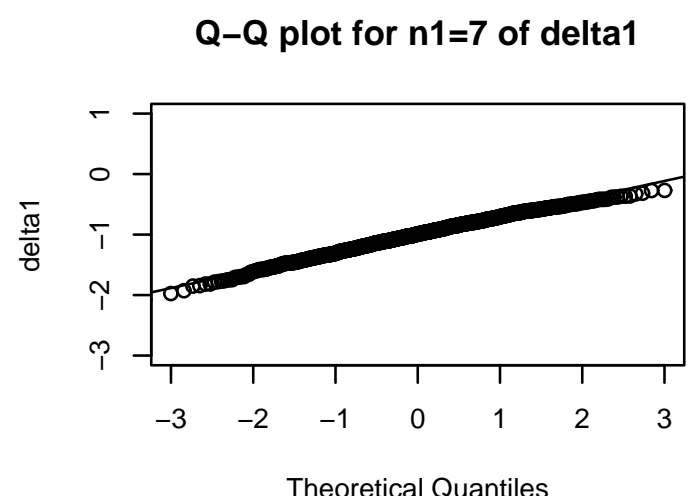

Theoretical Quantiles
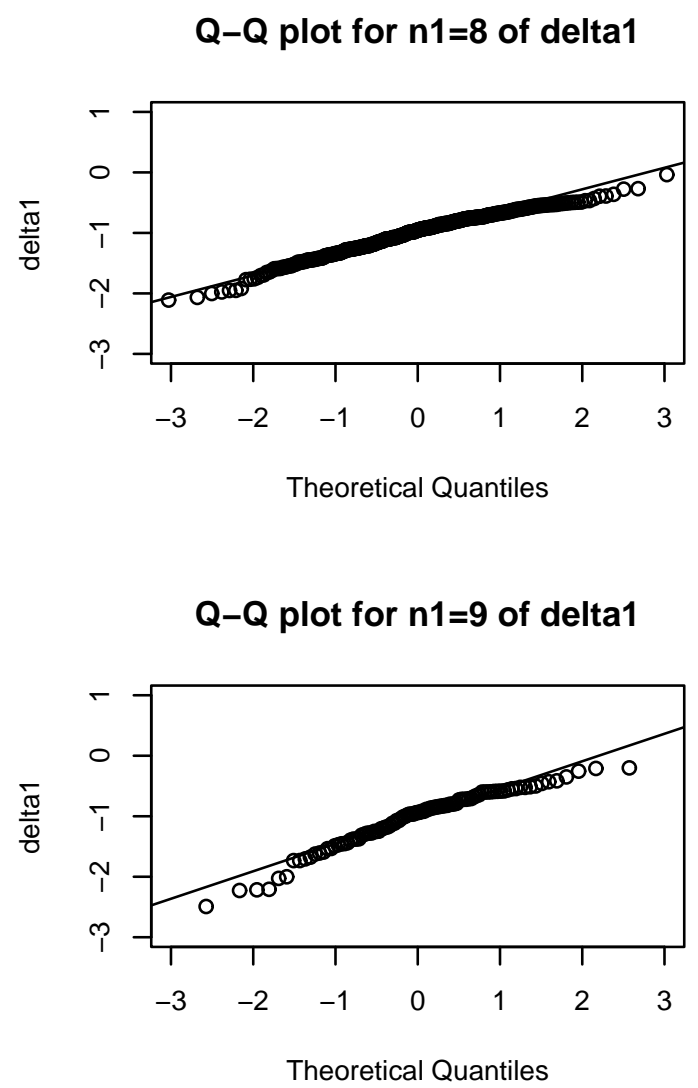
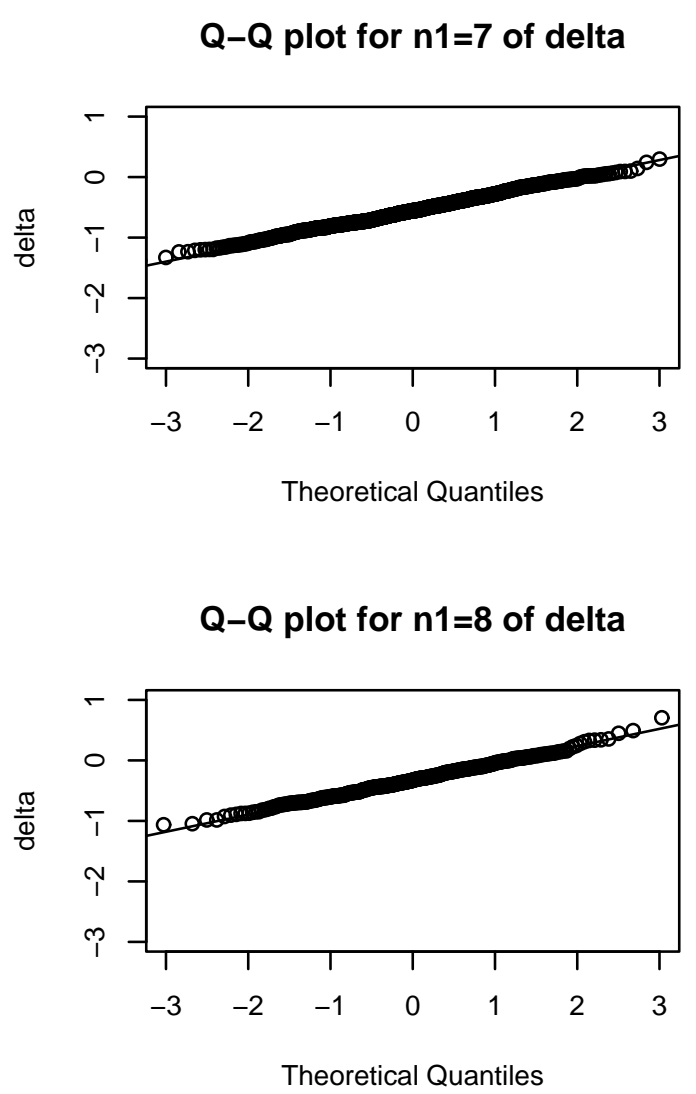

\section{$Q-Q$ plot for $n 1=9$ of delta}

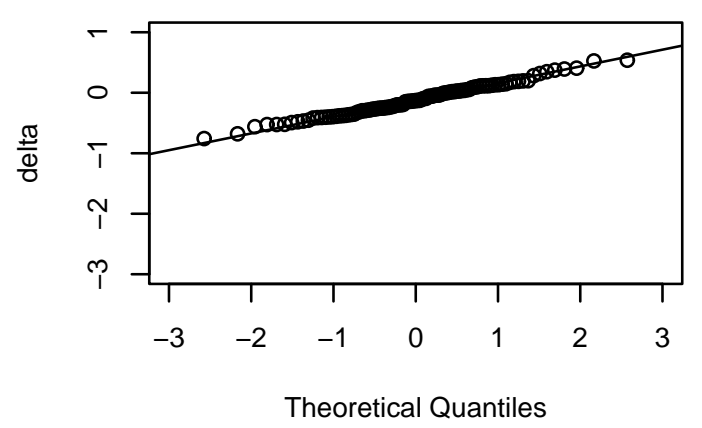

Figure 2.5: Normal Probability plots of $\hat{\Delta}_{1}$ and $\hat{\Delta}$ for perfect ranking when $n=10$ and $n_{1}=7,8,9$ 
perfect ranking with $\mathrm{n} 1=2$

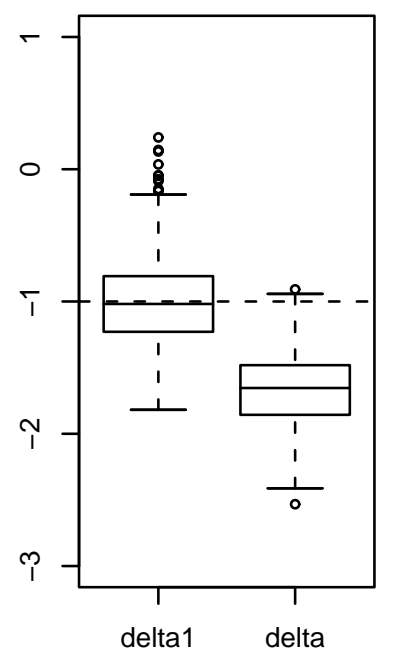

perfect ranking with $\mathrm{n} 1=7$

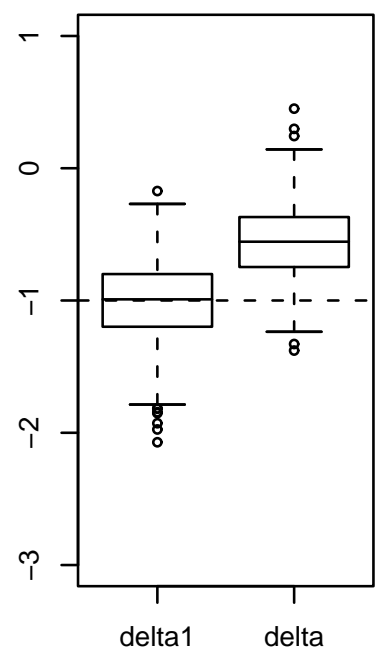

perfect ranking with $\mathrm{n} 1=4$

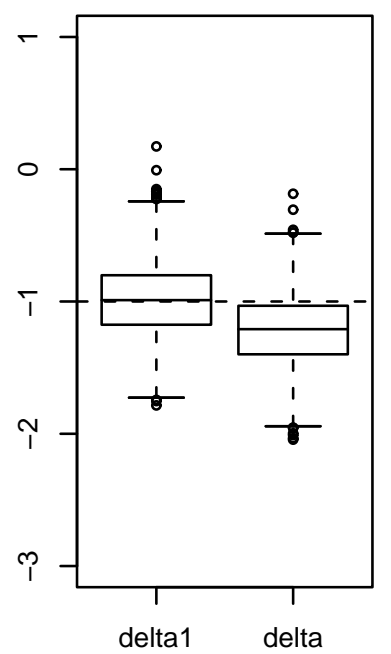

perfect ranking with $\mathrm{n} 1=9$

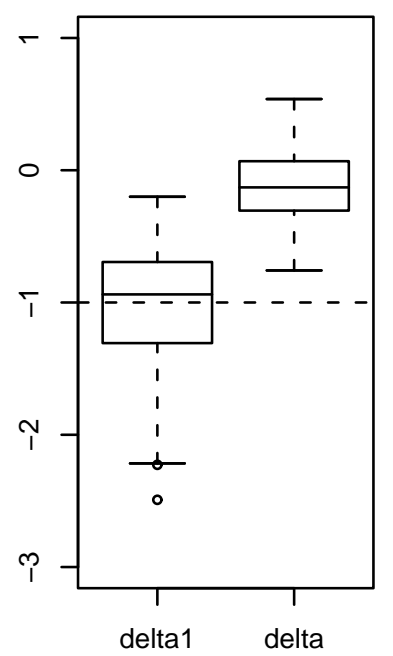

perfect ranking with $\mathrm{n} 1=5$

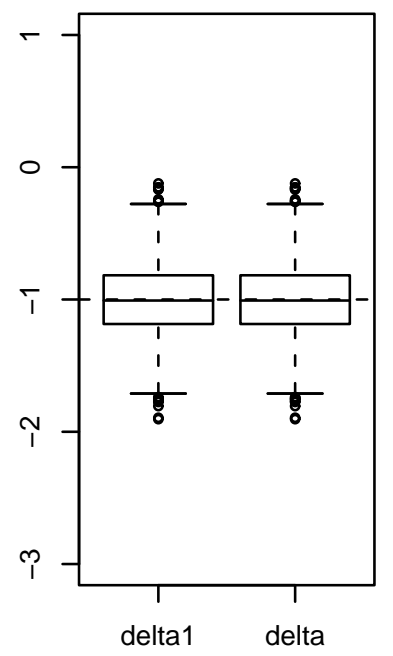

perfect ranking with $\mathrm{n}=10$

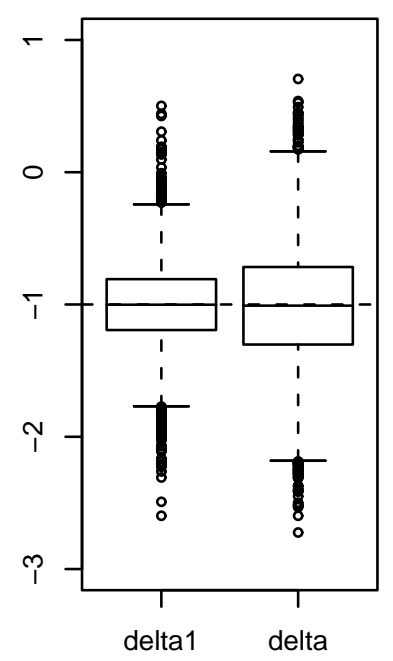

Figure 2.6: Box-plots of $\hat{\Delta}_{1}$ and $\hat{\Delta}$ for selected $n_{1}$ under perfect ranking 
tau=1 ranking with $\mathrm{n} 1=2$

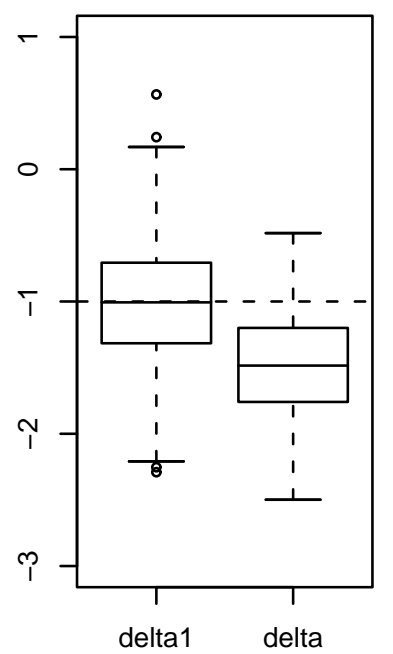

tau=1 ranking with $\mathrm{n} 1=7$

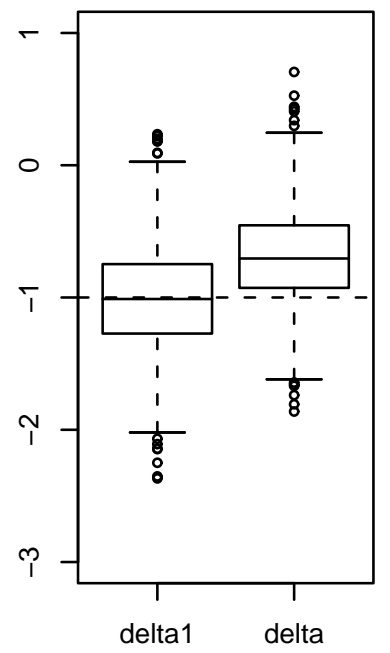

tau=1 ranking with $\mathrm{n} 1=4$

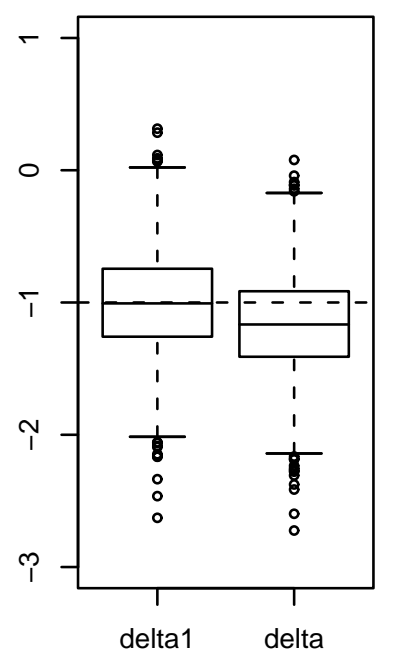

tau=1 ranking with $n 1=9$

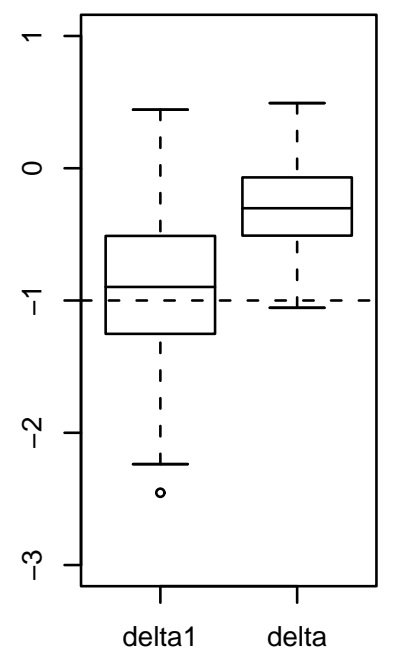

tau=1 ranking with $\mathrm{n} 1=5$

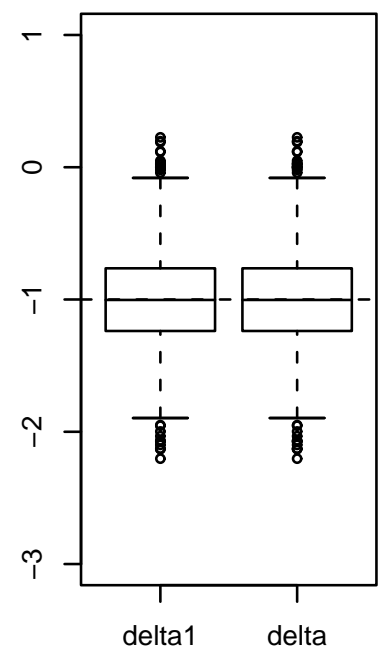

tau=1 ranking with $n=10$

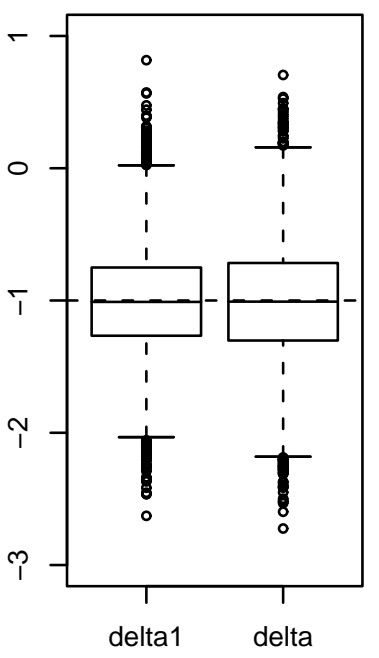

Figure 2.7: Box-plots of $\hat{\Delta}_{1}$ and $\hat{\Delta}$ for selected $n_{1}$ and $\tau^{2}=1$ 
tau=3 ranking with $\mathrm{n} 1=2$

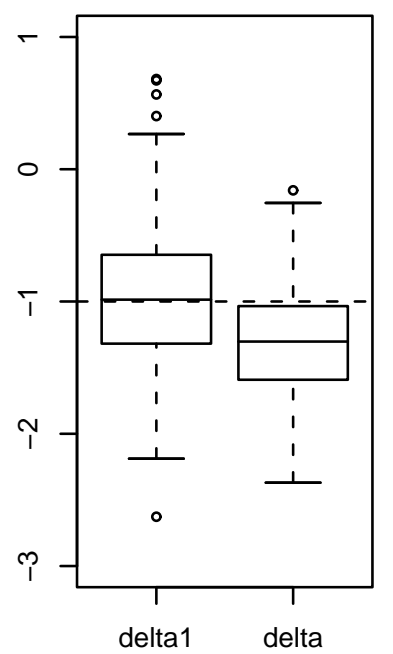

tau=3 ranking with $\mathrm{n} 1=\mathbf{7}$

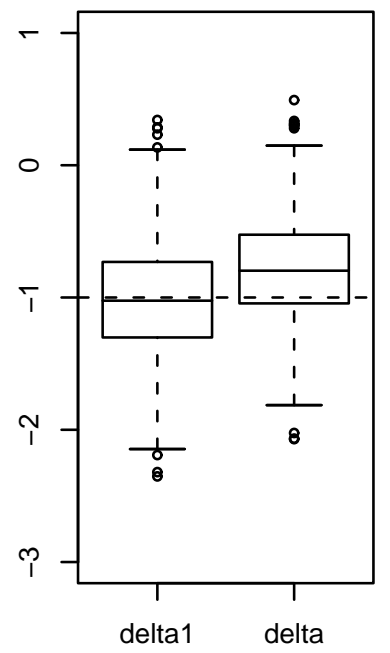

tau=3 ranking with $\mathrm{n} 1=4$

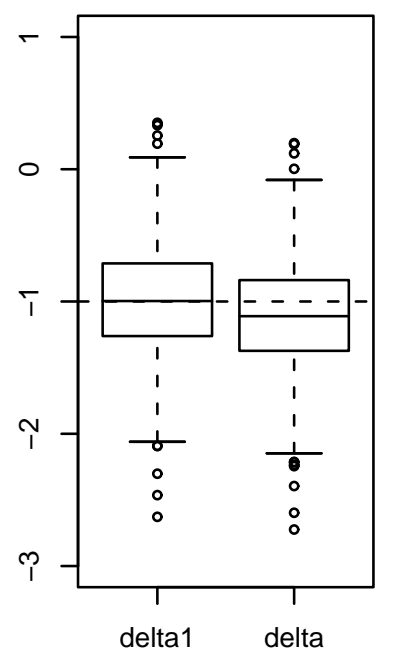

tau=3 ranking with $\mathrm{n} 1=9$

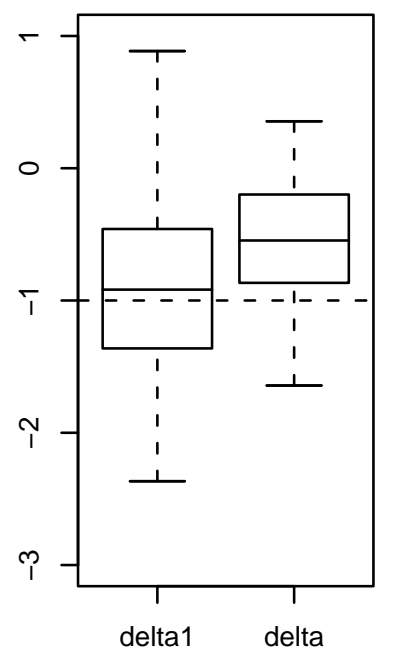

tau=3 ranking with $\mathrm{n} 1=5$

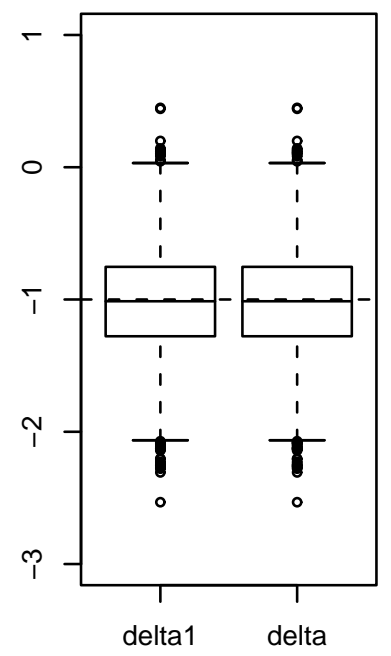

tau=3 ranking with $n=10$

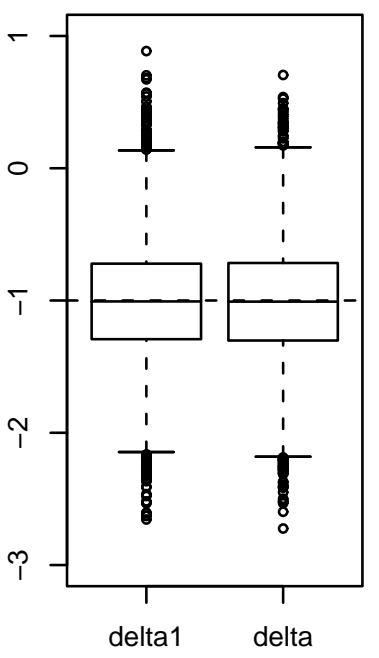

Figure 2.8: Box-plots of $\hat{\Delta}_{1}$ and $\hat{\Delta}$ for selected $n_{1}$ and $\tau^{2}=3$ 


\section{tau=100 ranking with $n 1=2$}

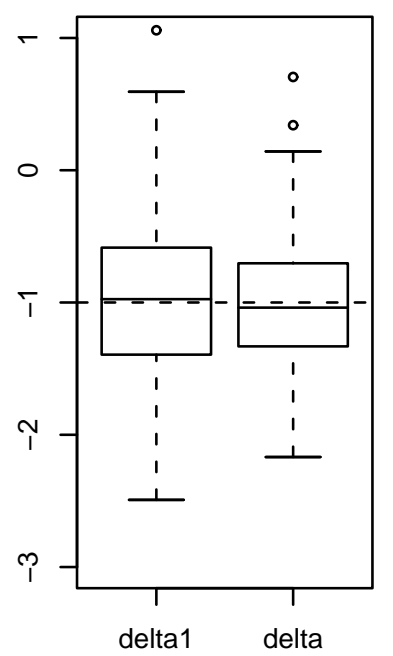

tau=100 ranking with $\mathrm{n} 1=7$

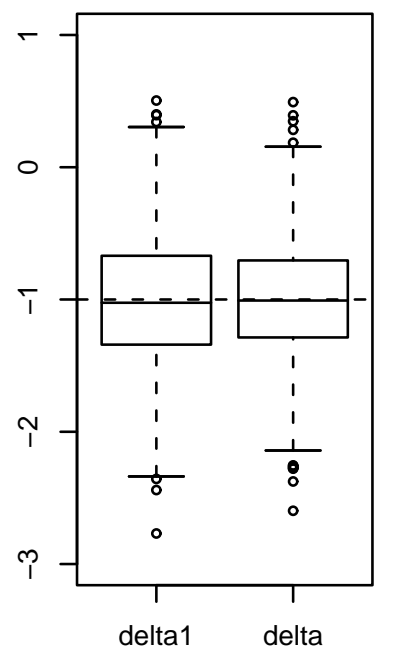

tau=100 ranking with $n 1=4$

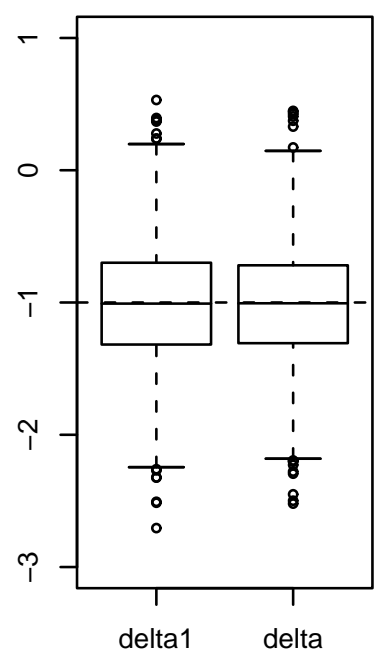

tau=100 ranking with $n 1=9$

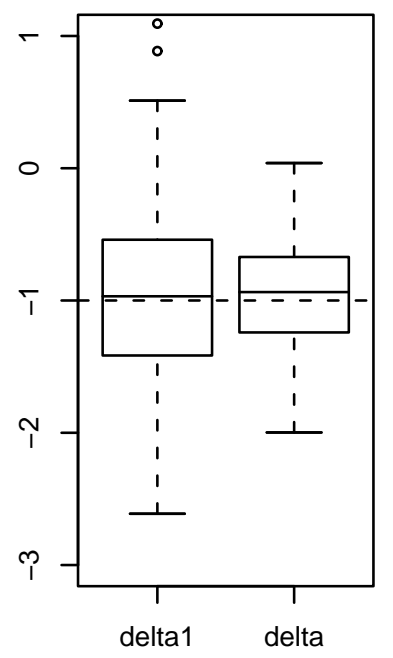

tau=100 ranking with $\mathrm{n} 1=5$

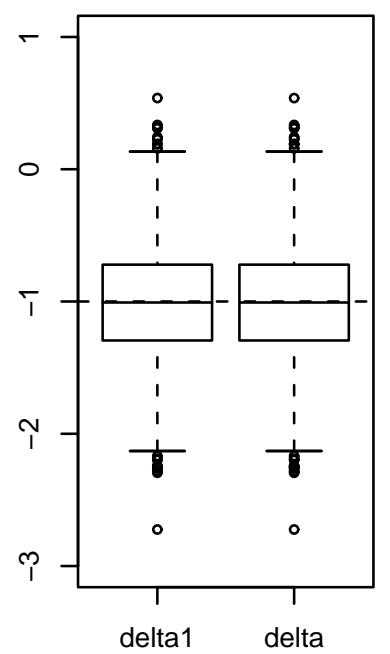

tau=100 ranking with $n=10$

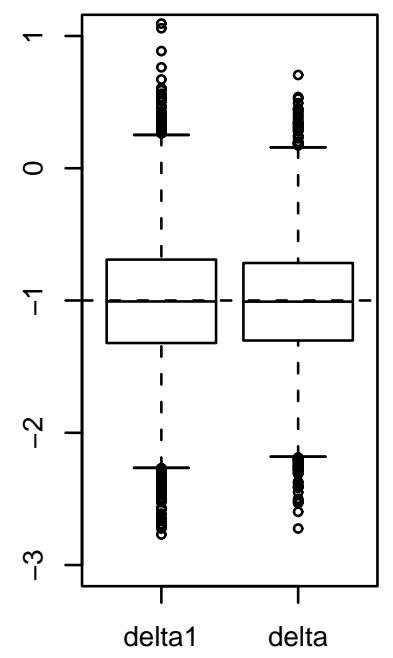

Figure 2.9: Box-plots of $\hat{\Delta}_{1}$ and $\hat{\Delta}$ for selected $n_{1}$ and $\tau^{2}=10000$ 


\begin{tabular}{ccccc}
\hline$\rho$ & $n$ & $M S E\left(\hat{\Delta}_{1}\right)$ & $M S E(\hat{\Delta})$ & $R E$ \\
\hline 1 & 10 & $0.0861(0.0014)$ & $0.1967(0.0028)$ & 2.2840 \\
& 30 & $0.0250(0.0004)$ & $0.0674(0.0009)$ & 2.6979 \\
& 50 & $0.0151(0.0002)$ & $0.0407(0.0006)$ & 2.6956 \\
& 100 & $0.0075(0.0001)$ & $0.0200(0.0003)$ & 2.6738 \\
\hline 0.7 & 10 & $0.1531(0.0024)$ & $0.1967(0.0028)$ & 1.2844 \\
& 30 & $0.0470(0.0007)$ & $0.0674(0.0009)$ & 1.4341 \\
& 50 & $0.0288(0.0004)$ & $0.0407(0.0006)$ & 1.4167 \\
& 100 & $0.0139(0.0002)$ & $0.0200(0.0003)$ & 1.4401 \\
\hline 0.5 & 10 & $0.1846(0.0087)$ & $0.2008(0.0090)$ & 1.0878 \\
& 30 & $0.0588(0.0026)$ & $0.0685(0.0031)$ & 1.1653 \\
& 50 & $0.0365(0.0016)$ & $0.0428(0.0018)$ & 1.1708 \\
& 100 & $0.0176(0.0008)$ & $0.0206(0.0009)$ & 1.1706 \\
\hline 0 & 10 & $0.2273(0.0035)$ & $0.1967(0.0028)$ & 0.8653 \\
& 30 & $0.0700(0.0010)$ & $0.0674(0.0009)$ & 0.9624 \\
& 50 & $0.0415(0.0006)$ & $0.0407(0.0006)$ & 0.9810 \\
& 100 & $0.0202(0.0003)$ & $0.0200(0.0003)$ & 0.9908 \\
\hline
\end{tabular}

Table 2.2: Mean square errors and relative efficiency of the estimators $\hat{\Delta}_{1}$ and $\hat{\Delta}$. Standard errors are given in parentheses. The simulation study is based on 10,000 replicates, $\mu_{1}-\mu_{2}=-1$ and $\sigma_{1}=\sigma_{2}=1$. 


\begin{tabular}{cccccc}
\hline$\rho$ & $n$ & $T_{n}\left(\hat{\Delta}_{1}\right)$ & $Z_{n}\left(\hat{\Delta}_{1}\right)$ & $S_{n}\left(\hat{\Delta}_{1}\right)$ & $T_{n}^{S R S}(\hat{\Delta})$ \\
\hline 1 & 10 & $0.0464(0.0021)$ & $0.1097(0.0031)$ & $0.0613(0.0024)$ & $0.0502(0.0022)$ \\
& 30 & $0.0497(0.0022)$ & $0.0616(0.0024)$ & $0.0494(0.0022)$ & $0.0479(0.0021)$ \\
& 50 & $0.0478(0.0021)$ & $0.0534(0.0022)$ & $0.0469(0.0021)$ & $0.0454(0.0021)$ \\
& 100 & $0.0483(0.0021)$ & $0.0516(0.0022)$ & $0.0486(0.0022)$ & $0.0489(0.0022)$ \\
\hline 0.7 & 10 & $0.0463(0.0021)$ & $0.1108(0.0031)$ & $0.0586(0.0023)$ & $0.0502(0.0022)$ \\
& 30 & $0.0490(0.0022)$ & $0.0606(0.0024)$ & $0.0477(0.0021)$ & $0.0479(0.0021)$ \\
& 50 & $0.0469(0.0021)$ & $0.0538(0.0023)$ & $0.0461(0.0021)$ & $0.0454(0.0021)$ \\
& 100 & $0.0518(0.0022)$ & $0.0560(0.0023)$ & $0.0528(0.0022)$ & $0.0489(0.0022)$ \\
\hline 0.5 & 10 & $0.0470(0.0021)$ & $0.1085(0.0031)$ & $0.0597(0.0024)$ & $0.0502(0.0022)$ \\
& 30 & $0.0528(0.0022)$ & $0.0625(0.0024)$ & $0.0502(0.0022)$ & $0.0479(0.0021)$ \\
& 50 & $0.0457(0.0021)$ & $0.0507(0.0022)$ & $0.0447(0.0021)$ & $0.0454(0.0021)$ \\
& 100 & $0.0487(0.0022)$ & $0.0516(0.0022)$ & $0.0484(0.0021)$ & $0.0489(0.0022)$ \\
\hline 0 & 10 & $0.0477(0.0021)$ & $0.1062(0.0031)$ & $0.0577(0.0023)$ & $0.0502(0.0022)$ \\
& 30 & $0.0483(0.0021)$ & $0.0632(0.0024)$ & $0.0501(0.0022)$ & $0.0479(0.0021)$ \\
& 50 & $0.0468(0.0021)$ & $0.0531(0.0022)$ & $0.0470(0.0021)$ & $0.0454(0.0021)$ \\
& 100 & $0.0494(0.0022)$ & $0.0519(0.0022)$ & $0.0492(0.0022)$ & $0.0489(0.0022)$ \\
\hline
\end{tabular}

Table 2.3: Estimated levels of a nominal $\alpha=0.05$ test for Student's $t$-distribution $\left(T_{n}\right)$ with $n-2$ d.f., Limiting normal distribution $\left(Z_{n}\right)$, Satterthwaite's approximation $\left(S_{n}\right)$ for $\hat{\Delta}_{1}$, and Student's $t$-distribution $\left(T_{n}^{S R S}\right)$ with $2 n-2$ d.f. for $\hat{\Delta}$. The simulation study is based on 10,000 replicates, $\sigma_{1}=\sigma_{2}=1$. Standard errors are given in parentheses. 


\begin{tabular}{cccccc}
\hline$\rho$ & $n_{1}$ & $T_{n}\left(\hat{\Delta}_{1}\right)$ & $Z_{n}\left(\hat{\Delta}_{1}\right)$ & $S_{n}\left(\hat{\Delta}_{1}\right)$ & $T_{n}^{S R S}(\hat{\Delta})$ \\
\hline 1 & 1,9 & $0.0387(0.0142)$ & NA (NA) & NA (NA) & $0.6354(0.0357)$ \\
& 2,8 & $0.0400(0.0067)$ & $0.2056(0.0138)$ & $0.1128(0.0108)$ & $0.2562(0.0150)$ \\
& 3,7 & $0.0436(0.0042)$ & $0.1201(0.0068)$ & $0.0627(0.0050)$ & $0.0545(0.0047)$ \\
& 4,6 & $0.0432(0.0032)$ & $0.0818(0.0043)$ & $0.0417(0.0031)$ & $0.0065(0.0012)$ \\
& 5 & $0.0506(0.0031)$ & $0.0795(0.0038)$ & $0.0429(0.0029)$ & $0.0000(0.0000)$ \\
\hline 0.7 & 1,9 & $0.0537(0.0156)$ & NA(NA) & NA (NA) & $0.2341(0.0296)$ \\
& 2,8 & $0.0556(0.0076)$ & $0.1967(0.0132)$ & $0.0889(0.0095)$ & $0.1722(0.0126)$ \\
& 3,7 & $0.0416(0.0041)$ & $0.1062(0.0063)$ & $0.0554(0.0047)$ & $0.0558(0.0047)$ \\
& 4,6 & $0.0458(0.0033)$ & $0.0866(0.0044)$ & $0.0436(0.0032)$ & $0.0297(0.0027)$ \\
& 5 & $0.0450(0.0030)$ & $0.0847(0.0040)$ & $0.0384(0.0028)$ & $0.0167(0.0018)$ \\
\hline 0.5 & 1,9 & $0.0315(0.0117)$ & NA (NA) & NA(NA) & $0.1261(0.0223)$ \\
& 2,8 & $0.0528(0.0077)$ & $0.1758(0.0130)$ & $0.0985(0.0102)$ & $0.0996(0.0102)$ \\
& 3,7 & $0.0438(0.0042)$ & $0.1078(0.0063)$ & $0.0508(0.0045)$ & $0.0553(0.0046)$ \\
& 4,6 & $0.0494(0.0034)$ & $0.0873(0.0044)$ & $0.0445(0.0032)$ & $0.0457(0.0033)$ \\
& 5 & $0.0453(0.0030)$ & $0.0822(0.0040)$ & $0.0403(0.0028)$ & $0.0277(0.0024)$ \\
\hline 0 & 1,9 & $0.0317(0.0127)$ & NA (NA) & NA(NA) & $0.0317(0.0127)$ \\
& 2,8 & $0.0487(0.0072)$ & $0.1670(0.0124)$ & $0.0885(0.0094)$ & $0.0431(0.0068)$ \\
& 3,7 & $0.0448(0.0043)$ & $0.1039(0.0064)$ & $0.0509(0.0046)$ & $0.0539(0.0047)$ \\
& 4,6 & $0.0490(0.0034)$ & $0.0863(0.0044)$ & $0.0412(0.0031)$ & $0.0502(0.0034)$ \\
& 5 & $0.0494(0.0031)$ & $0.0827(0.0039)$ & $0.0450(0.0029)$ & $0.0511(0.0031)$ \\
\hline & & & & &
\end{tabular}

Table 2.4: Estimated levels for a nominal level $\alpha=0.05$ test conditional on $n_{1}$ when sample size $n=10$. The simulation study is based on 10,000 replicates, $\sigma_{1}=\sigma_{2}=1$. Ranking quality varies from perfect ranking to random ranking. Standard errors are given in the parentheses. 


\begin{tabular}{cccccc}
\hline$\rho$ & $n_{1}$ & $T_{n}\left(\hat{\Delta}_{1}\right)$ & $Z_{n}\left(\hat{\Delta}_{1}\right)$ & $S_{n}\left(\hat{\Delta}_{1}\right)$ & $T_{n}^{S R S}(\hat{\Delta})$ \\
\hline 1 & $1: 3,17: 19$ & $0.0870(0.0550)$ & $0.1739(0.0789)$ & $0.0870(0.0550)$ & $0.9130(0.0550)$ \\
& $4: 6,14: 16$ & $0.0502(0.0064)$ & $0.0842(0.0081)$ & $0.0646(0.0072)$ & $0.2891(0.0132)$ \\
& $7: 9,11: 13$ & $0.0505(0.0026)$ & $0.0709(0.0031)$ & $0.0514(0.0026)$ & $0.0225(0.0018)$ \\
& 10 & $0.0587(0.0039)$ & $0.0682(0.0042)$ & $0.0536(0.0038)$ & $0.0011(0.0006)$ \\
\hline 0.7 & $1: 3,17: 19$ & $0.0400(0.0383)$ & $0.2400(0.0784)$ & $0.0800(0.0516)$ & $0.4000(0.0979)$ \\
& $4: 6,14: 16$ & $0.0446(0.0061)$ & $0.0752(0.0078)$ & $0.0446(0.0061)$ & $0.1731(0.0112)$ \\
& $7: 9,11: 13$ & $0.0485(0.0026)$ & $0.0654(0.0030)$ & $0.0476(0.0026)$ & $0.0389(0.0023)$ \\
& 10 & $0.0527(0.0037)$ & $0.0678(0.0041)$ & $0.0511(0.0036)$ & $0.0226(0.0024)$ \\
\hline 0.5 & $1: 3,17: 19$ & $0.0400(0.0388)$ & $0.1600(0.0700)$ & $0.1200(0.0629)$ & $0.1600(0.0732)$ \\
& $4: 6,14: 16$ & $0.0492(0.0065)$ & $0.0765(0.0080)$ & $0.0419(0.0060)$ & $0.1157(0.0096)$ \\
& $7: 9,11: 13$ & $0.0483(0.0025)$ & $0.0661(0.0029)$ & $0.0476(0.0025)$ & $0.0460(0.0025)$ \\
& 10 & $0.0533(0.0038)$ & $0.0647(0.0041)$ & $0.0516(0.0037)$ & $0.0358(0.0031)$ \\
\hline 0 & $1: 3,17: 19$ & $0.0000(0.0000)$ & $0.0500(0.0482)$ & $0.0000(0.0000)$ & $0.2000(0.0873)$ \\
& $4: 6,14: 16$ & $0.0363(0.0057)$ & $0.0688(0.0077)$ & $0.0447(0.0063)$ & $0.0447(0.0063)$ \\
& $7: 9,11: 13$ & $0.0543(0.0027)$ & $0.0736(0.0031)$ & $0.0531(0.0027)$ & $0.0541(0.0027)$ \\
& 10 & $0.0450(0.0035)$ & $0.0627(0.0041)$ & $0.0444(0.0035)$ & $0.0467(0.0036)$ \\
\hline
\end{tabular}

Table 2.5: Estimated levels for a nominal level $\alpha=0.05$ test conditional on grouped $n_{1}$ when sample size $n=20$. The simulation study is based on 10,000 replicates, $\sigma_{1}=$ $\sigma_{2}=1$. Ranking quality varies from perfect ranking to random ranking. Standard errors are given in the parentheses. 


\begin{tabular}{cccccc}
\hline$\rho$ & $n_{1}$ & $T_{n}\left(\hat{\Delta}_{1}\right)$ & $Z_{n}\left(\hat{\Delta}_{1}\right)$ & $S_{n}\left(\hat{\Delta}_{1}\right)$ & $T_{n}^{S R S}(\hat{\Delta})$ \\
\hline 1 & $1: 15,85: 99$ & $\mathrm{NA}(\mathrm{NA})$ & $\mathrm{NA}(\mathrm{NA})$ & $\mathrm{NA}(\mathrm{NA})$ & $\mathrm{NA}(\mathrm{NA})$ \\
& $16: 30,70: 84$ & $\mathrm{NA}(\mathrm{NA})$ & $\mathrm{NA}(\mathrm{NA})$ & $\mathrm{NA}(\mathrm{NA})$ & $\mathrm{NA}(\mathrm{NA})$ \\
& $31: 49,51: 69$ & $0.0474(0.002)$ & $0.0510(0.002)$ & $0.0478(0.002)$ & $0.0526(0.002)$ \\
& 50 & $0.0589(0.006)$ & $0.0589(0.006)$ & $0.0589(0.006)$ & $0.0013(0.001)$ \\
\hline 0.7 & $1: 15,85: 99$ & $\mathrm{NA}(\mathrm{NA})$ & $\mathrm{NA}(\mathrm{NA})$ & $\mathrm{NA}(\mathrm{NA})$ & $\mathrm{NA}(\mathrm{NA})$ \\
& $16: 30,70: 84$ & $\mathrm{NA}(\mathrm{NA})$ & $\mathrm{NA}(\mathrm{NA})$ & $\mathrm{NA}(\mathrm{NA})$ & $\mathrm{NA}(\mathrm{NA})$ \\
& $31: 49,51: 69$ & $0.0527(0.002)$ & $0.0569(0.002)$ & $0.0538(0.002)$ & $0.0519(0.002)$ \\
& 50 & $0.0412(0.005)$ & $0.0451(0.005)$ & $0.0412(0.005)$ & $0.0129(0.003)$ \\
\hline 0.5 & $1: 15,85: 99$ & $\mathrm{NA}(\mathrm{NA})$ & $\mathrm{NA}(\mathrm{NA})$ & $\mathrm{NA}(\mathrm{NA})$ & $\mathrm{NA}(\mathrm{NA})$ \\
& $16: 30,70: 84$ & $\mathrm{NA}(\mathrm{NA})$ & $\mathrm{NA}(\mathrm{NA})$ & $\mathrm{NA}(\mathrm{NA})$ & $\mathrm{NA}(\mathrm{NA})$ \\
& $31: 49,51: 69$ & $0.0488(0.002)$ & $0.0518(0.002)$ & $0.0485(0.002)$ & $0.0511(0.002)$ \\
& 50 & $0.0470(0.005)$ & $0.0496(0.005)$ & $0.0470(0.005)$ & $0.0229(0.004)$ \\
\hline 0 & $1: 15,85: 99$ & $\mathrm{NA}(\mathrm{NA})$ & $\mathrm{NA}(\mathrm{NA})$ & $\mathrm{NA}(\mathrm{NA})$ & $\mathrm{NA}(\mathrm{NA})$ \\
& $16: 30,70: 84$ & $\mathrm{NA}(\mathrm{NA})$ & $\mathrm{NA}(\mathrm{NA})$ & $\mathrm{NA}(\mathrm{NA})$ & $\mathrm{NA}(\mathrm{NA})$ \\
& $31: 49,51: 69$ & $0.0489(0.002)$ & $0.0513(0.002)$ & $0.0487(0.002)$ & $0.0481(0.002)$ \\
& 50 & $0.0558(0.006)$ & $0.0597(0.006)$ & $0.0558(0.006)$ & $0.0584(0.006)$ \\
\hline
\end{tabular}

Table 2.6: Estimated levels for a nominal level $\alpha=0.05$ test conditional on grouped $n_{1}$ when sample size $n=100$. The simulation study is based on 10,000 replicates, $\sigma_{1}=$ $\sigma_{2}=1$. Ranking quality varies from perfect ranking to random ranking. Standard errors are given in parentheses. 

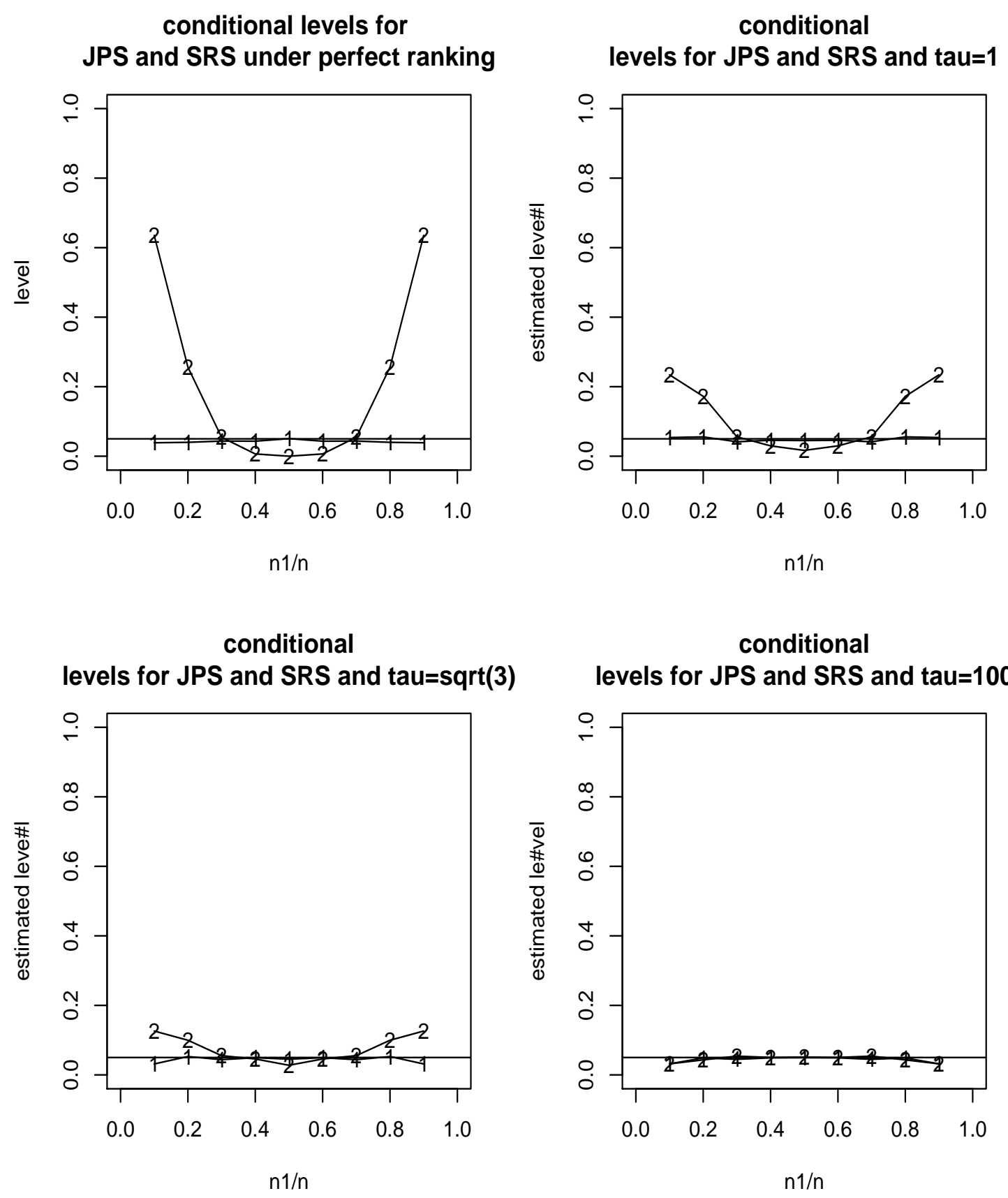

Figure 2.10: Estimated levels of a nominal level $\alpha=0.05$ test for $T_{n}$, Student's $t$ distribution with $n-2$ d.f. and for $T_{n}^{S R S}$, Student's $t$-distribution with $2 n-2$ d.f. conditional on $n_{1}$. Sample size $n=10$. 

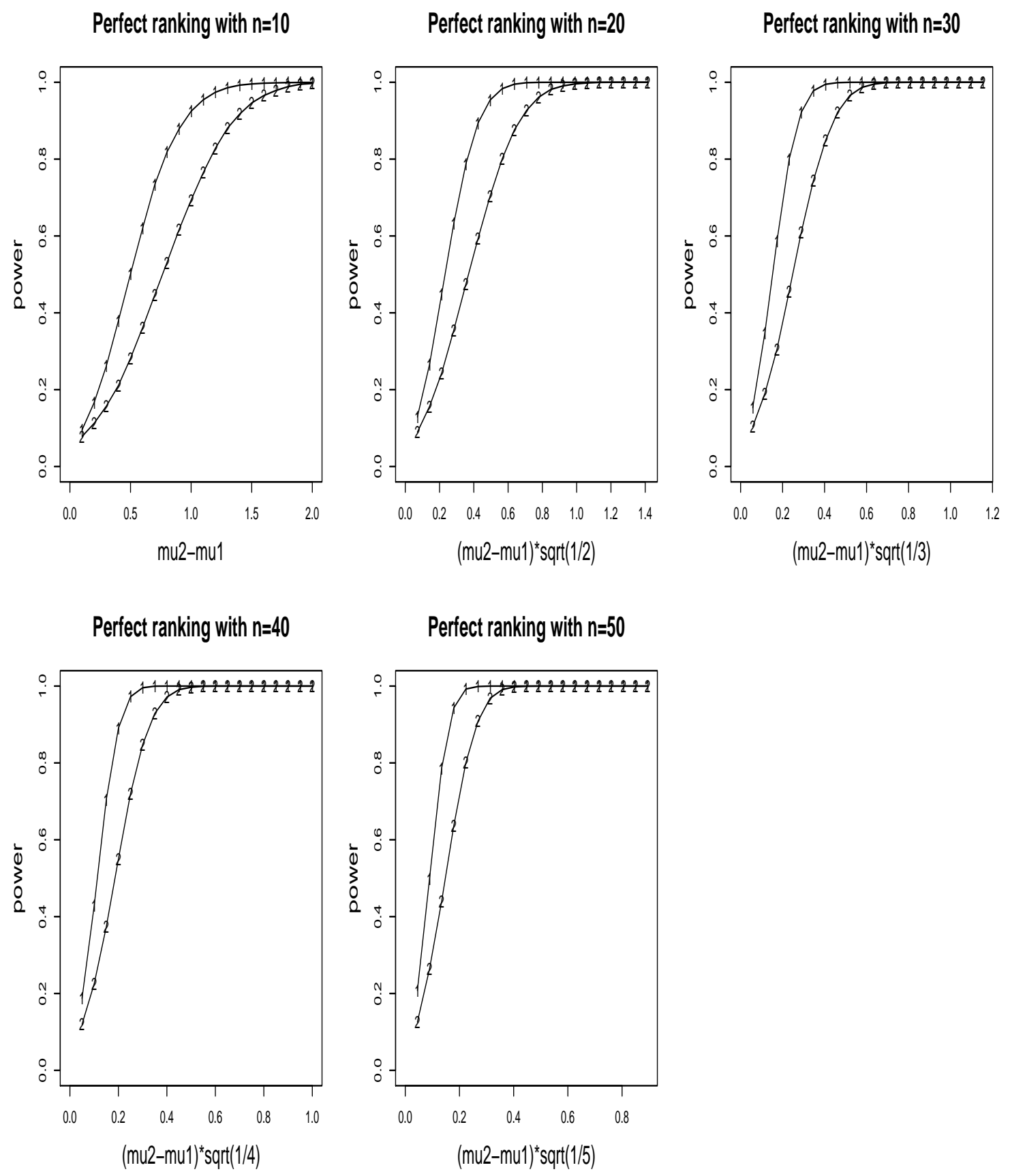

Figure 2.11: Empirical power of $T_{n}$ with $n-2$ d.f. (with symbol 1) and $T_{n}^{S R S}$ with $2 n-2$ d.f. (with symbol 2 ) under perfect ranking. 

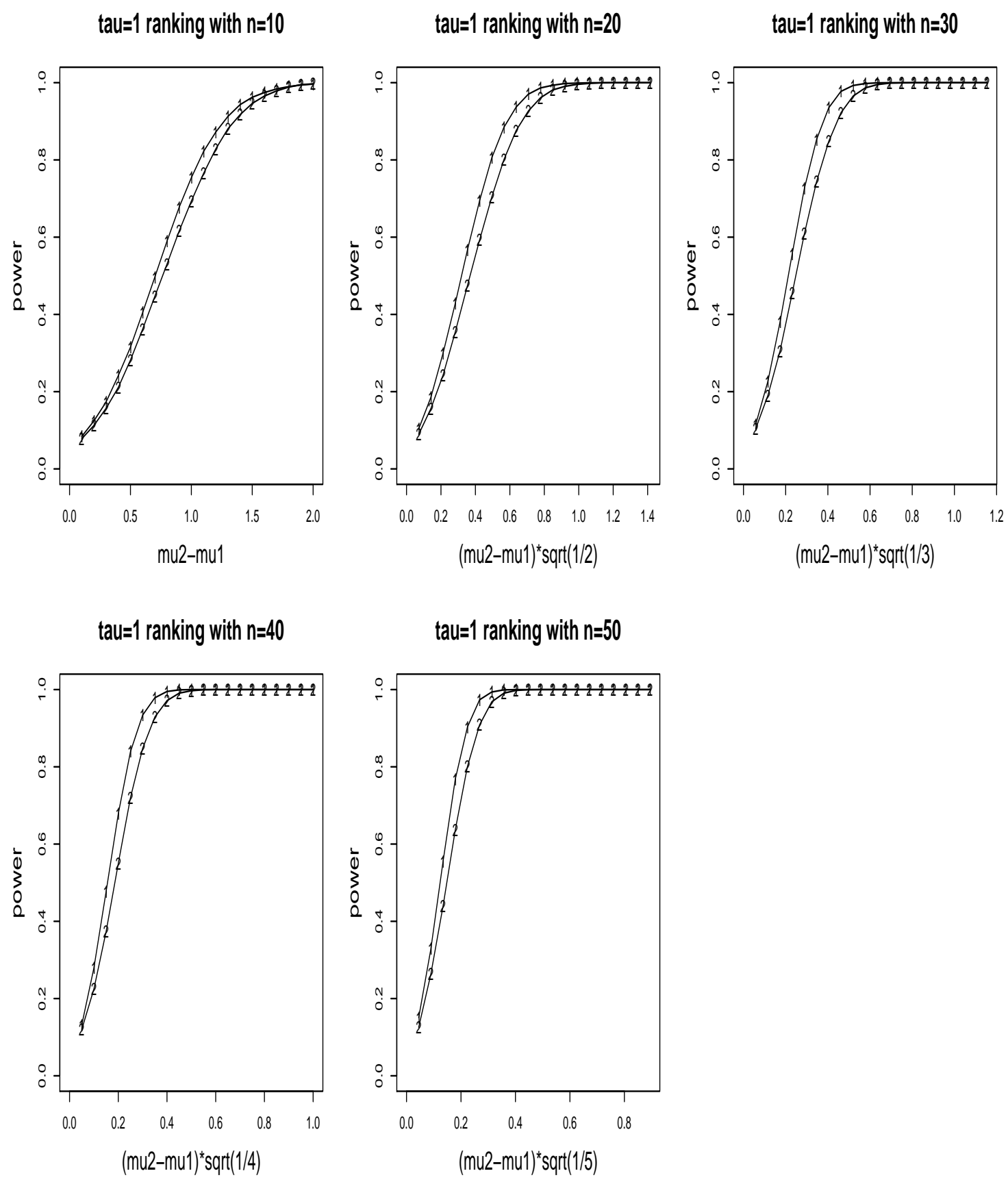

Figure 2.12: Empirical power of $T_{n}$ with $n-2$ d.f. (with symbol 1) and $T_{n}^{S R S}$ with $2 n-2$ d.f. (with symbol 2) when $\tau^{2}=1$. 

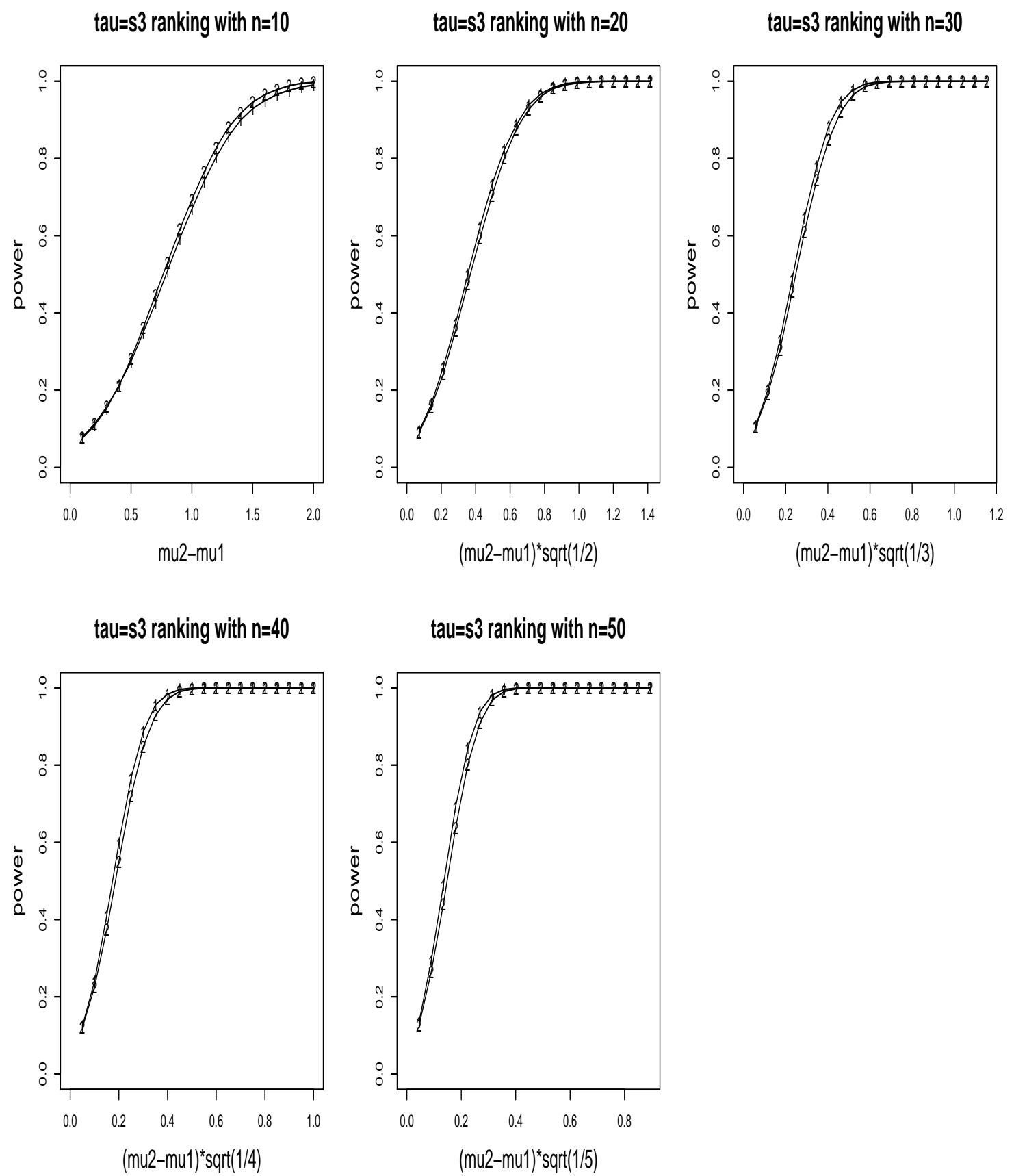

Figure 2.13: Empirical power of $T_{n}$ with $n-2$ d.f. (with symbol 1) and $T_{n}^{S R S}$ with $2 n-2$ d.f. (with symbol 2) when $\tau^{2}=3$. 

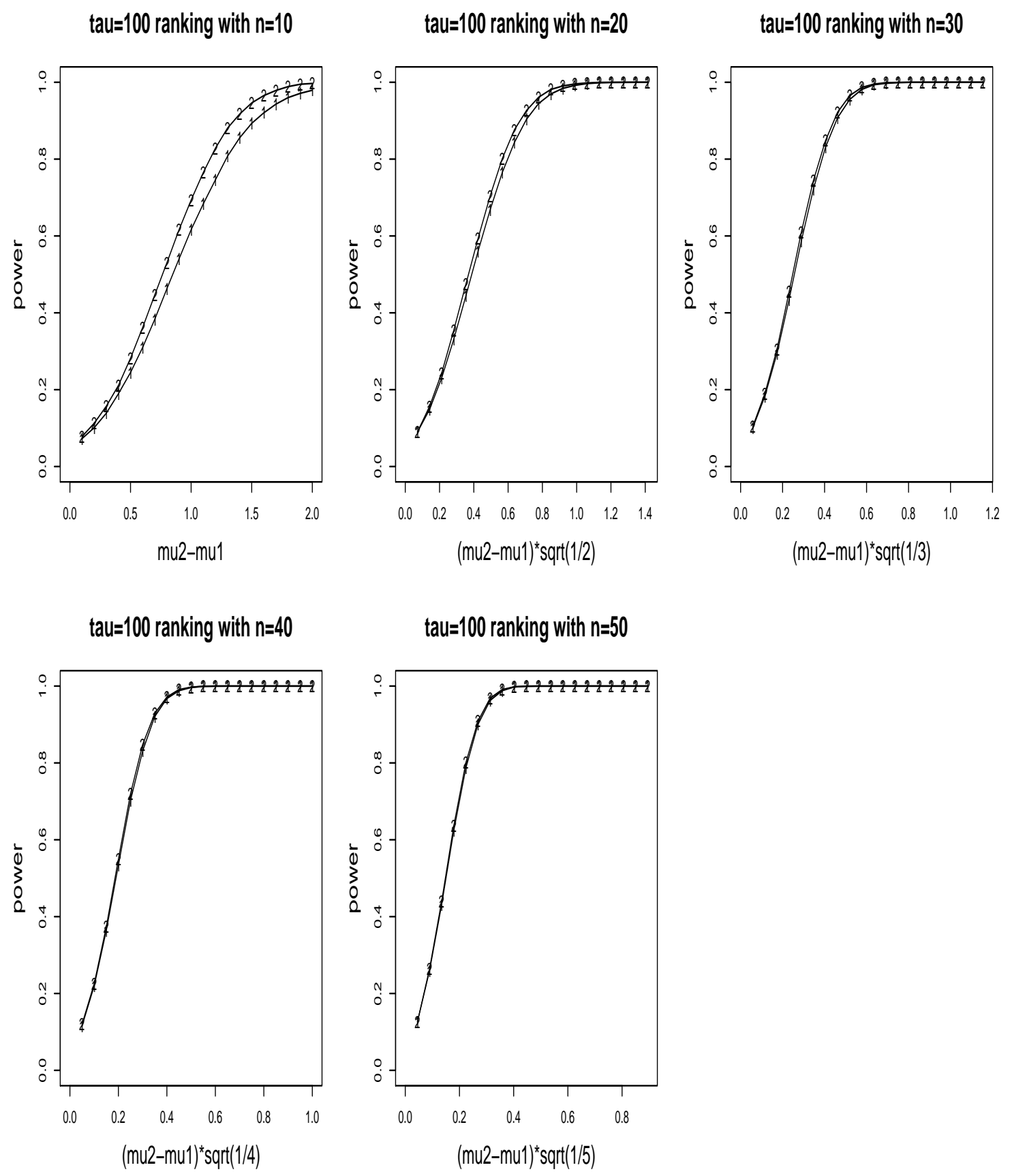

Figure 2.14: Empirical power of $T_{n}$ with $n-2$ d.f. (with symbol 1) and $T_{n}^{S R S}$ with $2 n-2$ d.f. (with symbol 2 ) when $\tau^{2}=10,000$. 


\begin{tabular}{cccccc}
\hline$\rho$ & $n$ & $T_{n}\left(\hat{\Delta}_{1}\right)$ & $Z_{n}\left(\hat{\Delta}_{1}\right)$ & $T_{n}^{S R S}(\hat{\Delta})$ & $Z_{n}^{S R S}(\hat{\Delta})$ \\
\hline 1 & 10 & $0.0491(0.0022)$ & $0.1090(0.0031)$ & $0.0577(0.0023)$ & $0.0749(0.0026)$ \\
& 30 & $0.0515(0.0022)$ & $0.0642(0.0025)$ & $0.0558(0.0023)$ & $0.0617(0.0024)$ \\
& 50 & $0.0502(0.0022)$ & $0.0565(0.0023)$ & $0.0480(0.0021)$ & $0.0502(0.0022)$ \\
& 100 & $0.0471(0.0021)$ & $0.0489(0.0022)$ & $0.0494(0.0022)$ & $0.0512(0.0022)$ \\
\hline 0.7 & 10 & $0.0515(0.0022)$ & $0.1138(0.0032)$ & $0.0577(0.0023)$ & $0.0749(0.0026)$ \\
& 30 & $0.0497(0.0022)$ & $0.0617(0.0024)$ & $0.0558(0.0023)$ & $0.0617(0.0024)$ \\
& 50 & $0.0488(0.0022)$ & $0.0555(0.0023)$ & $0.0480(0.0021)$ & $0.0502(0.0022)$ \\
& 100 & $0.0499(0.0022)$ & $0.0533(0.0022)$ & $0.0494(0.0022)$ & $0.0512(0.0022)$ \\
\hline 0.5 & 10 & $0.0491(0.0022)$ & $0.1125(0.0032)$ & $0.0577(0.0023)$ & $0.0749(0.0026)$ \\
& 30 & $0.0495(0.0022)$ & $0.0645(0.0025)$ & $0.0558(0.0023)$ & $0.0617(0.0024)$ \\
& 50 & $0.0462(0.0021)$ & $0.0531(0.0022)$ & $0.0480(0.0021)$ & $0.0502(0.0022)$ \\
& 100 & $0.0505(0.0022)$ & $0.0538(0.0023)$ & $0.0494(0.0022)$ & $0.0512(0.0022)$ \\
\hline 0 & 10 & $0.0474(0.0021)$ & $0.1093(0.0031)$ & $0.0577(0.0023)$ & $0.0749(0.0026)$ \\
& 30 & $0.0506(0.0022)$ & $0.0635(0.0024)$ & $0.0558(0.0023)$ & $0.0617(0.0024)$ \\
& 50 & $0.0453(0.0021)$ & $0.0514(0.0022)$ & $0.0480(0.0021)$ & $0.0502(0.0022)$ \\
& 100 & $0.0481(0.0021)$ & $0.0499(0.0022)$ & $0.0494(0.0022)$ & $0.0512(0.0022)$ \\
\hline
\end{tabular}

Table 2.7: Estimated levels of a nominal $\alpha=0.05$ test for Student's $t$-distribution $\left(T_{n}\right)$ with $n-2$ d.f., Limiting normal distribution $\left(Z_{n}\right)$ for $\hat{\Delta}_{1}$, and for Student's $t$ distribution $\left(T_{n}^{S R S}\right)$ with $2 n-2$ d.f. and approximate normal distribution $\left(Z_{n}^{S R S}\right)$ for $\hat{\Delta}$. Simulation is based on 10,000 replicates, scale parameters for the two treatments are set to 1 and 4 respectively. Standard errors are given in parentheses. 

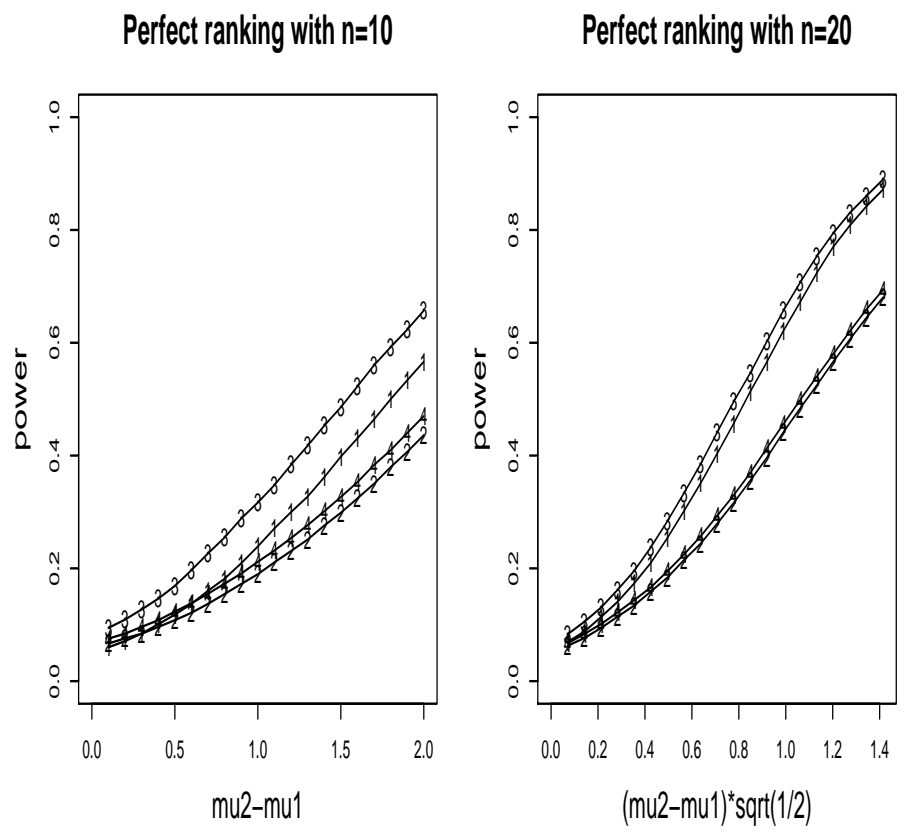

Perfect ranking with $n=30$
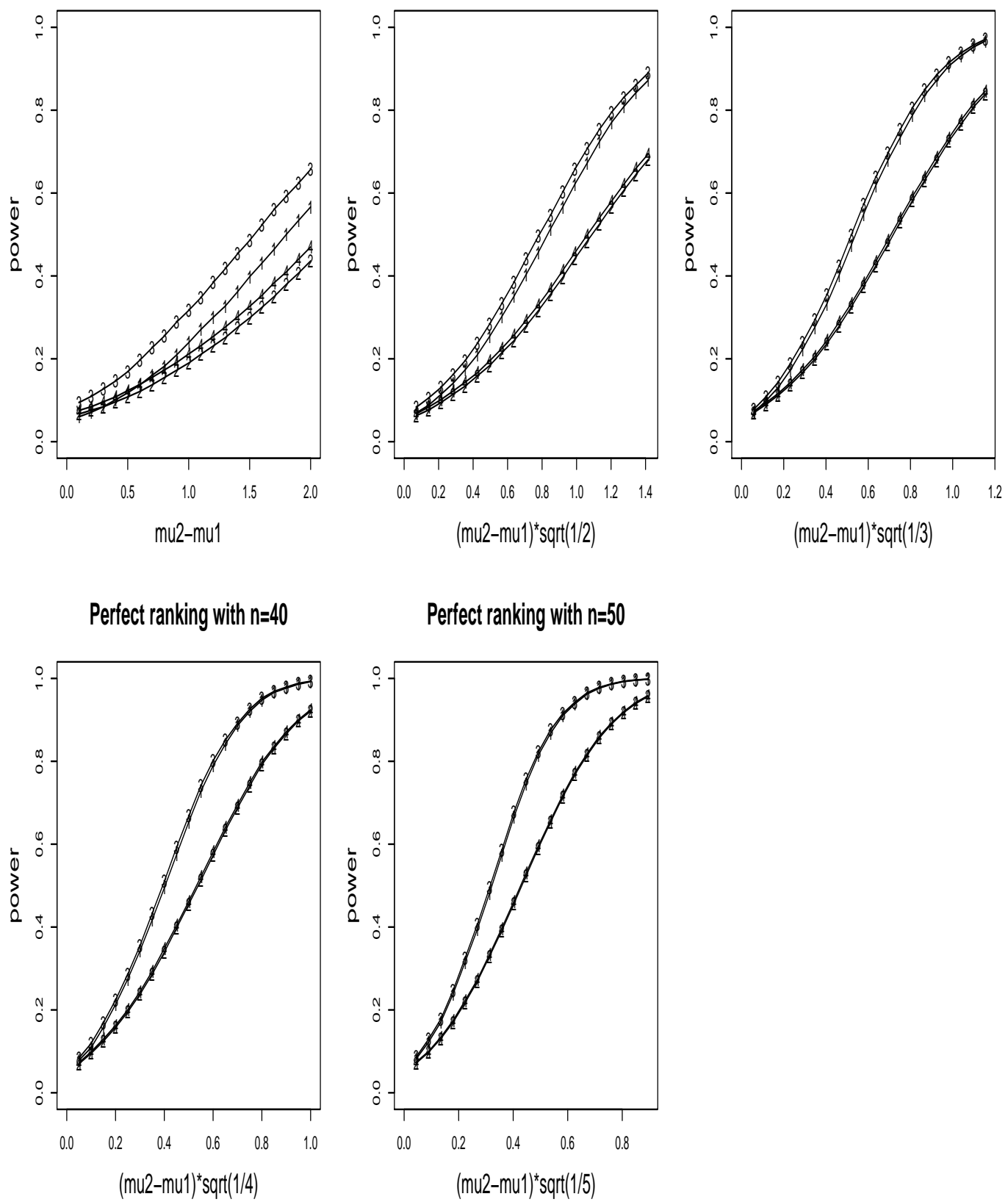

Figure 2.15: Empirical power of test statistics for $\hat{\Delta}_{1}$ including $t$-distribution with $n-2$ d.f. and normal approximation (indicated by labels 1 and 3 respectively) and test statistics for $\hat{\Delta}$ including $t$-distribution with $2 n-2$ d.f. and normal approximation (indicated by labels 2 and 4 respectively) under perfect ranking. The simulation is based on 10,000 replicates. Scale parameters for the two treatments are set to be 1 and 4 respectively. 


\begin{tabular}{cccccc}
\hline$\sigma$ & $n$ & $T_{n}\left(\hat{\Delta}_{1}\right)$ & $Z_{n}\left(\hat{\Delta}_{1}\right)$ & $T_{n}^{S R S}(\hat{\Delta})$ & $Z_{n}^{S R S}(\hat{\Delta})$ \\
\hline 0.5 & 10 & $0.0423(0.0020)$ & $0.1027(0.0030)$ & $0.0460(0.0021)$ & $0.0590(0.0024)$ \\
& 30 & $0.0460(0.0021)$ & $0.0601(0.0024)$ & $0.0473(0.0021)$ & $0.0521(0.0022)$ \\
& 50 & $0.0440(0.0021)$ & $0.0496(0.0022)$ & $0.0467(0.0021)$ & $0.0498(0.0022)$ \\
& 100 & $0.0432(0.0020)$ & $0.0457(0.0021)$ & $0.0462(0.0021)$ & $0.0475(0.0021)$ \\
\hline 1 & 10 & $0.0369(0.0019)$ & $0.087(0.0028)$ & $0.0332(0.0018)$ & $0.0475(0.0021)$ \\
& 30 & $0.0371(0.0019)$ & $0.0512(0.0022)$ & $0.0413(0.0020)$ & $0.0465(0.0021)$ \\
& 50 & $0.0382(0.0019)$ & $0.0452(0.0021)$ & $0.0424(0.0020)$ & $0.0447(0.0021)$ \\
& 100 & $0.0401(0.0020)$ & $0.0449(0.0021)$ & $0.0439(0.0020)$ & $0.0450(0.0021)$ \\
\hline 2 & 10 & $0.0295(0.0017)$ & $0.0542(0.0023)$ & $0.0149(0.0012)$ & $0.0245(0.0015)$ \\
& 30 & $0.0225(0.0015)$ & $0.0337(0.0018)$ & $0.0218(0.0015)$ & $0.0254(0.0016)$ \\
& 50 & $0.0245(0.0015)$ & $0.0289(0.0017)$ & $0.0253(0.0016)$ & $0.0278(0.0016)$ \\
& 100 & $0.0251(0.0016)$ & $0.0269(0.0016)$ & $0.0261(0.0016)$ & $0.0273(0.0016)$ \\
\hline 4 & 10 & $0.0231(0.0015)$ & $0.0250(0.0016)$ & $0.0034(0.0006)$ & $0.0072(0.0008)$ \\
& 30 & $0.0096(0.0010)$ & $0.0115(0.0011)$ & $0.0049(0.0007)$ & $0.0056(0.0007)$ \\
& 50 & $0.0084(0.0009)$ & $0.0096(0.0010)$ & $0.0064(0.0008)$ & $0.0071(0.0008)$ \\
& 100 & $0.0092(0.0010)$ & $0.0099(0.0010)$ & $0.0091(0.0009)$ & $0.0101(0.0010)$ \\
\hline
\end{tabular}

Table 2.8: Estimated levels of a nominal $\alpha=0.05$ test for Student's $t$-distribution $\left(T_{n}\right)$ with $n-2$ d.f., Limiting normal distribution $\left(Z_{n}\right)$ for $\hat{\Delta}_{1}$, and for Student's $t$-distribution $\left(T_{n}^{S R S}\right)$ with $2 n-2$ d.f. and approximate normal distribution $\left(Z_{n}^{S R S}\right)$ for $\hat{\Delta}$ under perfect ranking. Simulation is based on 10,000 replicates, $\sigma_{1}=\sigma_{2}$. The underlying distribution for $\epsilon$ is lognormal with mean of $\log (\epsilon)$ set to 0 and standard deviation of $\log (\epsilon)$ to be $0.5,1,2,4$ respectively. Standard errors are given in parentheses. 


\begin{tabular}{cccccc}
\hline d.f. & $n$ & $T_{n}\left(\hat{\Delta}_{1}\right)$ & $Z_{n}\left(\hat{\Delta}_{1}\right)$ & $T_{n}^{S R S}(\hat{\Delta})$ & $Z_{n}^{S R S}(\hat{\Delta})$ \\
\hline 5 & 10 & $0.0447(0.0021)$ & $0.1127(0.0032)$ & $0.0476(0.0021)$ & $0.0616(0.0024)$ \\
& 30 & $0.0470(0.0021)$ & $0.0588(0.0024)$ & $0.0472(0.0021)$ & $0.0522(0.0022)$ \\
& 50 & $0.0483(0.0021)$ & $0.0527(0.0022)$ & $0.0476(0.0021)$ & $0.0504(0.0022)$ \\
& 100 & $0.0474(0.0021)$ & $0.0500(0.0022)$ & $0.0490(0.0022)$ & $0.0504(0.0022)$ \\
\hline 10 & 10 & $0.0492(0.0022)$ & $0.1158(0.0032)$ & $0.0499(0.0022)$ & $0.0682(0.0025)$ \\
& 30 & $0.0443(0.0021)$ & $0.0585(0.0023)$ & $0.0469(0.0021)$ & $0.0524(0.0022)$ \\
& 50 & $0.0501(0.0022)$ & $0.0584(0.0023)$ & $0.0519(0.0022)$ & $0.0545(0.0023)$ \\
& 100 & $0.0465(0.0021)$ & $0.0488(0.0022)$ & $0.0531(0.0022)$ & $0.0543(0.0023)$ \\
\hline 20 & 10 & $0.0476(0.0021)$ & $0.1174(0.0032)$ & $0.0526(0.0022)$ & $0.0672(0.0025)$ \\
& 30 & $0.0493(0.0022)$ & $0.0622(0.0024)$ & $0.0495(0.0022)$ & $0.0537(0.0023)$ \\
& 50 & $0.0473(0.0021)$ & $0.0557(0.0023)$ & $0.0492(0.0022)$ & $0.0522(0.0022)$ \\
& 100 & $0.0485(0.0021)$ & $0.0506(0.0022)$ & $0.0524(0.0022)$ & $0.0538(0.0023)$ \\
\hline 40 & 10 & $0.0502(0.0022)$ & $0.1095(0.0031)$ & $0.0452(0.0021)$ & $0.0595(0.0024)$ \\
& 30 & $0.0475(0.0021)$ & $0.0585(0.0023)$ & $0.0479(0.0021)$ & $0.0526(0.0022)$ \\
& 50 & $0.0445(0.0021)$ & $0.0508(0.0022)$ & $0.0468(0.0021)$ & $0.0493(0.0022)$ \\
& 100 & $0.0515(0.0022)$ & $0.0544(0.0023)$ & $0.0512(0.0022)$ & $0.0524(0.0022)$ \\
\hline
\end{tabular}

Table 2.9: Estimated level of a nominal $\alpha=0.05$ test for Student's $t$-distribution $\left(T_{n}\right)$ with $n-2$ d.f., Limiting normal distribution $\left(Z_{n}\right)$ for $\hat{\Delta}_{1}$, and for Student's $t$-distribution $\left(T_{n}^{S R S}\right)$ with $2 n-2$ d.f. and approximate normal distribution $\left(Z_{n}^{S R S}\right)$ for $\hat{\Delta}$ under perfect ranking. Simulation is based on 10,000 replicates, $\sigma_{1}=\sigma_{2}$. The underlying distributions for $\epsilon$ are $t$ with degree of freedom 5, 10, 20, 40. Standard errors are given in parentheses. 


\section{CHAPTER 3}

\section{RAO-BLACKWELLIZED ESTIMATORS}

The estimator $\hat{\Delta}_{1}$ depends on the specific pairing and ranking of the units, and so requires only $n$ rankings. We might choose to collect more information about the ranks of the units. The extreme is that the ranker who is qualified to rank the experimental units has the ability to rank all the possible pairs of the units where one unit is from treatment and the other one is from control. In this situation, when the sample sizes for treatment and control are both $n$, the number of all possible pairs that need to be ranked is $n^{2}$. Alternatively, if the units can be ranked from 1 to $2 n$, all of the pairwise rankings can be determined.

\subsection{The Rao-Blackwellization of the JPS Estimator}

When additional ranking information is available, we can apply the Rao-Blackwell Theorem to obtain a better unbiased estimator of $\Delta$. In this research, we describe two Rao-Blackwellized estimators, $\hat{\Delta}_{2}$ and $\hat{\Delta}_{3}$. The estimator $\hat{\Delta}_{2}$ is the Rao-

Blackwellization of $\hat{\Delta}_{1}$ from the unconditional point of view. The estimator $\hat{\Delta}_{3}$ is obtained by applying a Taylor series expansion on a four dimension random vector and is defined as vector Rao-Blackwellized estimator. 


\subsubsection{The Rao-Blackwellized Estimator}

The estimator $\hat{\Delta}_{2}$ is defined as the average, over all possible pairings, of $\hat{\Delta}_{1}$. Denote the ranking result for the pair of $Y_{1 i}-Y_{2 j}$ by $R_{i j}$, let $d_{i j}=Y_{1 i}-Y_{2 j}$, and let $S$ denote the collection of sets of Type 1, so that

$$
R_{i j}= \begin{cases}1 & \text { when }\left(Y_{1 i}, Y_{2 j}\right) \in S \\ 0 & \text { when }\left(Y_{1 i}, Y_{2 j}\right) \notin S,\end{cases}
$$

$i=1, \cdots, n, j=1, \cdots, n$. There are $n !$ equally likely pairings of the units. Define

$$
\hat{\Delta}_{2}=E\left(\hat{\Delta}_{1} \mid Y_{11}, \cdots, Y_{2 n}, R_{11}, \cdots, R_{n n}\right)=\frac{1}{n !} \sum_{l=1}^{n !} \hat{\Delta}_{1 l},
$$

where the average is taken over the $n$ ! permutations. We refer to the estimator as the Rao-Blackwellized estimator (RBE).

By the Rao-Blackwell Theorem we have

$$
\begin{aligned}
\operatorname{Var}\left(\hat{\Delta}_{1}\right)= & \operatorname{Var}\left(E\left(\hat{\Delta}_{1} \mid Y_{11}, \cdots, Y_{2 n}, R_{11}, \cdots, R_{n n}\right)\right) \\
& +E\left(\operatorname{Var}\left(\hat{\Delta}_{1} \mid Y_{11}, \cdots, Y_{2 n}, R_{11}, \cdots, R_{n n}\right)\right) \\
= & \operatorname{Var}\left(\hat{\Delta}_{2}\right)+E\left(\operatorname{Var}\left(\hat{\Delta}_{1} \mid Y_{11}, \cdots, Y_{2 n}, R_{11}, \cdots, R_{n n}\right)\right) .
\end{aligned}
$$

Hence, $\operatorname{Var}\left(\hat{\Delta}_{1}\right) \geq \operatorname{Var}\left(\hat{\Delta}_{2}\right)$.

To reduce the computational burden of computing $n$ ! estimates or to alleviate the difficulty in ranking all pairs of units, one might select a relatively small subset of pairings, averaging estimators of the form $\hat{\Delta}_{1}$ over these pairings alone. Define $B$ as the number of pairings in this subset. For these partial Rao-Blackwellizations,

we can establish improvement over $\hat{\Delta}_{1}$ in the same fashion. Define $\hat{\Delta}_{2}^{*}$ as partial Rao-Blackwellized estimator (PRBE). We have

$$
\hat{\Delta}_{2}^{*}=\frac{1}{B} \sum_{l=1}^{B} \hat{\Delta}_{1 l}
$$

where $B$ is fixed and $B \leq n$ !. 


\subsubsection{The Vector Rao-Blackwellized Estimator}

The estimator $\hat{\Delta}_{3}$ arises from a Rao-Blackwellization of a vector from which $\hat{\Delta}_{1}$ is constructed. The randomization and ranking procedure of the JPS estimator $\hat{\Delta}_{1}$ can be described equivalently as choosing $n$ sets from the $n^{2}$ possible sets with the restriction that each unit appears in exactly one set. Let $n_{1}$ represent the number of Type 1 sets and let $S_{n}$ be the collection of these Type 1 sets among the $n$ selected sets. Define the four-dimensional random vector $\mathbf{W}_{i j}=\left[d_{i j}, 0,1,0\right]^{T}$ if $R_{i j} \in S$ and $\left[0, d_{i j}, 0,1\right]^{T}$ otherwise. Then the average of these $\mathbf{W}_{i j}$ 's can be expressed as

$$
\mathbf{W}_{n}=\left[\frac{n_{1}}{n} \frac{1}{n_{1}} \sum_{d_{i j} \in S_{n}} d_{i j}, \frac{n-n_{1}}{n} \frac{1}{n-n_{1}} \sum_{d_{i j} \notin S_{n}} d_{i j}, \frac{n_{1}}{n}, \frac{n-n_{1}}{n}\right]^{T} .
$$

Define $\delta_{1}=E\left(d_{i j} \mid R_{i j}=1\right)$ and $\delta_{2}=E\left(d_{i j} \mid R_{i j}=0\right)$. The expectation of $\mathbf{W}_{n}$, conditional on $n_{1}$ is

$$
E\left(\mathbf{W}_{n} \mid n_{1}\right)=\left[\frac{n_{1}}{n} \delta_{1}, \frac{n-n_{1}}{n} \delta_{2}, \frac{n_{1}}{n}, \frac{n-n_{1}}{n}\right]^{T} .
$$

Our target is $\Delta=\mu_{1}-\mu_{2}=\frac{1}{2}\left(\delta_{1}+\delta_{2}\right)$. Let $G(x)=\frac{1}{2}\left(\frac{x_{1}}{x_{3}}+\frac{x_{2}}{x_{4}}\right)$, where $x=$ $\left[x_{1}, x_{2}, x_{3}, x_{4}\right]^{T}, G\left(\mathbf{W}_{n}\right)=\hat{\Delta}_{1}$. A Taylor series expansion yields

$$
G(x)=G\left(x_{0}\right)+G^{\prime}\left(x_{0}\right)\left(x-x_{0}\right)+o\left(x-x_{0}\right),
$$

where $G^{\prime}\left(x_{0}\right)$ is the first order derivative evaluated at $x_{0}$. Replacing $x$ and $x_{0}$ by (3.1) and (3.2) respectively, we then have

$$
\begin{aligned}
G(x) & =\frac{1}{2}\left(\frac{1}{n_{1}} \sum_{d_{i j} \in S_{n}} d_{i j}+\frac{1}{n-n_{1}} \sum_{d_{i j} \notin S_{n}} d_{i j}\right), \\
G\left(x_{0}\right) & =\frac{1}{2}\left(\delta_{1}+\delta_{2}\right)=\mu_{1}-\mu_{2}, \\
G^{\prime}\left(x_{0}\right) & =0.5\left[\frac{1}{n_{1} / n}, \frac{1}{\left(n-n_{1}\right) / n},-\frac{\delta_{1}}{n_{1} / n},-\frac{\delta_{2}}{\left(n-n_{1}\right) / n}\right] .
\end{aligned}
$$


By Taylor series expansion, it is clear that $G(x)=G\left(\mathbf{W}_{n}\right)=\hat{\Delta}_{1}$ is an asymptotically unbiased estimator of $G\left(x_{0}\right)=G\left(E\left(\mathbf{W}_{n} \mid n_{1}\right)\right)=\mu_{1}-\mu_{2}$, with variance matrix $G^{\prime} \Sigma_{n} G^{\prime T}$, where $\operatorname{Var}\left(\mathbf{W}_{n}\right)=\Sigma_{n}$

The Rao-Blackwellized estimator of $\mathbf{W}_{n}$ can be expressed as the expectation of $\mathbf{W}_{n}$ conditional on the responses $Y_{11}, Y_{12}, \cdots, Y_{2 n}$ and all the pairings and rankings $R_{i j} ; i=1,2, \cdots, n, j=1,2, \cdots, n$. Let $m_{1}$ represent the number of Type 1 sets in the collection of $n^{2}$ sets. Then

$$
\begin{aligned}
\mathbf{W} & =E\left(\mathbf{W}_{n} \mid Y_{11}, \cdots, Y_{2 n}, R_{11}, \cdots, R_{n n}\right) \\
& =\left[\frac{m_{1}}{n^{2}} \frac{1}{m_{1}} \sum_{d_{i j} \in S} d_{i j}, \frac{n^{2}-m_{1}}{n^{2}} \frac{1}{n^{2}-m_{1}} \sum_{d_{i j} \notin S} d_{i j}, \frac{m_{1}}{n^{2}}, \frac{n^{2}-m_{1}}{n^{2}}\right]^{T} .
\end{aligned}
$$

Define $\hat{\Delta}_{3}=G(\mathbf{W})$. The corresponding expectation of $\mathbf{W}$ is

$$
E\left(\mathbf{W} \mid m_{1}\right)=\left[\frac{m_{1}}{n^{2}} \delta_{1}, \frac{n^{2}-m_{1}}{n^{2}} \delta_{2}, \frac{m_{1}}{n^{2}}, \frac{n^{2}-m_{1}}{n^{2}}\right]^{T}
$$

Applying the Taylor series expansion with $G(x)=\frac{1}{2}\left(\frac{x_{1}}{x_{3}}+\frac{x_{2}}{x_{4}}\right)$ and replacing $x$ and $x_{0}$ by $\mathbf{W}$ and $E\left(\mathbf{W} \mid m_{1}\right)$, respectively, leads us to conclude that

$$
\frac{1}{2}\left(\frac{1}{m_{1}} \sum_{d_{i j} \in S} d_{i j}+\frac{1}{n^{2}-m_{1}} \sum_{d_{i j} \notin S} d_{i j}\right)
$$

is an asymptotically unbiased estimator of $\mu_{1}-\mu_{2}$ with variance matrix $G^{\prime} \Sigma_{n^{2}} G^{\prime T}$, where $\operatorname{Var}(\mathbf{W})=\Sigma_{n^{2}}$.

By the Rao-Blackwell Theorem, we have

$$
\operatorname{Var}\left(\mathbf{W}_{n}\right)=\operatorname{Var}(\mathbf{W})+E\left(\operatorname{Var}\left(\mathbf{W}_{n} \mid Y_{11}, \cdots, Y_{2 n}, R_{11}, \cdots, R_{n n}\right)\right)
$$

Hence $\Sigma_{n}$ is the sum of $\Sigma_{n^{2}}$ and a non-negative definite matrix, and so $\operatorname{Var}\left(\hat{\Delta}_{3}\right)=$ $G^{\prime} \Sigma_{n^{2}} G^{\prime T} \leq G^{\prime} \Sigma_{n} G^{\prime T}=\operatorname{Var}\left(\hat{\Delta}_{1}\right)$. We refer to $\hat{\Delta}_{3}$ as the vector Rao-Blackwellized estimator (VRBE). 
When the sample size is $n$, we need to average over $n$ ! possible $\hat{\Delta}_{1}$ 's to obtain the RBE $\hat{\Delta}_{2}$. As $n$ gets larger, this number grows extremely quickly. Thus for large sample sizes, we may encounter computational difficulty. In this case, we consider an average over only $B<n$ ! permutations to reduce computation greatly without sacrificing too much efficiency. On the other hand, computation of $\hat{\Delta}_{3}$ is trivial. Table 3.1 shows that, when rankings are of value, VRBE has smaller MSE than $\hat{\Delta}_{1}$, $\hat{\Delta}_{2}$ or SRS. Moreover, this estimator can also be extended for use with unbalanced designs. We prefer VRBE to RBE for both computational efficiency and estimation accuracy. We prefer both to $\hat{\Delta}_{1}$, and we prefer all of the new estimators to $\hat{\Delta}$.

\subsubsection{An Illustrative Example for $\hat{\Delta}_{1}, \hat{\Delta}_{2}$, and $\hat{\Delta}_{3}$}

So far, we have proposed three new estimators, $\hat{\Delta}_{1}, \hat{\Delta}_{2}$, and $\hat{\Delta}_{3}$, to adjust for the difference in EUs when extra information on the EUs is available. To illustrate the procedure for calculating the new estimators, we take a simple example with a data set of sample size $n=4$. In particular, the treatment group and control group both have 4 observations.

For $\hat{\Delta}_{1}$, we randomly arrange the 4 EUs in the treatment group and control group respectively. Then these 8 EUs form 4 sets with each set having one treatment and one control unit. For illustration, we list one such possible arrangement in Table 3.2. Let $u$ denotes the EU. As we can see from the table, the 4 sets here are $\left\{\left(u_{11}, u_{21}\right),\left(u_{12}, u_{22}\right),\left(u_{13}, u_{23}\right),\left(u_{14}, u_{24}\right)\right\}$. To obtain $\hat{\Delta}_{1}$, we rank the units within each set using the available extra information. Without loss of generality, we assume that the rankings for the four pairs are $\left\{u_{11}<u_{21}, u_{12}>u_{22}, u_{13}<u_{23}, u_{14}>u_{24}\right\}$. 


\begin{tabular}{cccccc}
\hline$\rho$ & $n$ & $M S E\left(\hat{\Delta}_{1}\right)$ & $M S E\left(\hat{\Delta}_{2}\right)$ & $M S E\left(\hat{\Delta}_{3}\right)$ & $M S E(\hat{\Delta})$ \\
\hline 1 & 5 & $0.2812(0.0165)$ & $0.1687(0.0128)$ & $0.1047(0.0094)$ & $0.4032(0.0186)$ \\
& 7 & $0.1622(0.0094)$ & $0.0692(0.0044)$ & $0.0549(0.0025)$ & $0.2831(0.0119)$ \\
& 10 & $0.0774(0.0036)$ & $0.0379(0.0026)$ & $0.0356(0.0017)$ & $0.1905(0.0086)$ \\
& 30 & $0.0250(0.0011)$ & $0.0104(0.0005)$ & $0.0106(0.0005)$ & $0.0697(0.0033)$ \\
& 50 & $0.0134(0.0006)$ & $0.0060(0.0003)$ & $0.0060(0.0003)$ & $0.0379(0.0016)$ \\
\hline 0.7 & 5 & $0.4071(0.0189)$ & $0.3068(0.0149)$ & $0.2845(0.0136)$ & $0.4032(0.0186)$ \\
& 7 & $0.2291(0.0102)$ & $0.1801(0.0077)$ & $0.1729(0.0074)$ & $0.2831(0.0119)$ \\
& 10 & $0.1538(0.0066)$ & $0.1197(0.0053)$ & $0.1185(0.0052)$ & $0.1905(0.0086)$ \\
& 30 & $0.0481(0.0022)$ & $0.0408(0.0019)$ & $0.0408(0.0019)$ & $0.0697(0.0033)$ \\
& 50 & $0.0259(0.0011)$ & $0.0219(0.0009)$ & $0.0219(0.0009)$ & $0.0379(0.0016)$ \\
\hline 0.5 & 5 & $0.4524(0.0206)$ & $0.3731(0.0174)$ & $0.3641(0.0169)$ & $0.4032(0.0186)$ \\
& 7 & $0.2887(0.0126)$ & $0.2370(0.0102)$ & $0.2343(0.0101)$ & $0.2831(0.0119)$ \\
& 10 & $0.1860(0.0081)$ & $0.1620(0.0070)$ & $0.1611(0.0070)$ & $0.1905(0.0086)$ \\
& 30 & $0.0603(0.0028)$ & $0.0562(0.0026)$ & $0.0563(0.0026)$ & $0.0697(0.0033)$ \\
& 50 & $0.0322(0.0014)$ & $0.0298(0.0013)$ & $0.0298(0.0013)$ & $0.0379(0.0016)$ \\
\hline 0 & 5 & $0.5053(0.0232)$ & $0.4423(0.0205)$ & $0.4532(0.0207)$ & $0.4032(0.0186)$ \\
& 7 & $0.3261(0.0150)$ & $0.2922(0.0125)$ & $0.2931(0.0126)$ & $0.2831(0.0119)$ \\
& 10 & $0.2227(0.0106)$ & $0.2005(0.0091)$ & $0.1994(0.0090)$ & $0.1905(0.0086)$ \\
& 30 & $0.0723(0.0034)$ & $0.0707(0.0033)$ & $0.0706(0.0033)$ & $0.0697(0.0033)$ \\
& 50 & $0.0387(0.0017)$ & $0.0381(0.0016)$ & $0.0381(0.0016)$ & $0.0379(0.0016)$ \\
\hline & & & & &
\end{tabular}

Table 3.1: Mean square errors of the estimators $\hat{\Delta}_{1}, \hat{\Delta}_{2}, \hat{\Delta}_{3}$, and $\hat{\Delta}$. Standard errors are given in the parentheses. The simulation study is based on 10000 replicates, $\mu_{1}-\mu_{2}=-1$ and $\sigma_{1}=\sigma_{2}=1$.

\begin{tabular}{ccccccc}
\hline Set & Rank & Treatment & Response & Rank & Treatment & Response \\
\hline 1 & 1 & $\mathrm{C}$ & $Y_{11}$ & 2 & $\mathrm{~T}$ & $Y_{21}$ \\
2 & 2 & $\mathrm{C}$ & $Y_{12}$ & 1 & $\mathrm{~T}$ & $Y_{22}$ \\
3 & 1 & $\mathrm{C}$ & $Y_{13}$ & 2 & $\mathrm{~T}$ & $Y_{23}$ \\
4 & 2 & $\mathrm{C}$ & $Y_{14}$ & 1 & $\mathrm{~T}$ & $Y_{24}$ \\
\hline
\end{tabular}

Table 3.2: One possible arrangements of the data with sample size equal to 4 and the rankings for the four pairs are $\left\{u_{11}<u_{21} ; u_{12}>u_{22}, u_{13}<u_{23} ; u_{14}>u_{24}\right\}$. 
Then the JPS estimator $\hat{\Delta}_{1}$ can be expressed as

$$
\hat{\Delta}_{1}^{(1)}=\frac{1}{2}\left\{\frac{1}{2}\left[\left(Y_{11}-Y_{21}\right)+\left(Y_{13}-Y_{23}\right)\right]+\frac{1}{2}\left[\left(Y_{12}-Y_{22}\right)+\left(Y_{14}-Y_{24}\right)\right]\right\} .
$$

The RBE $\hat{\Delta}_{2}$ is defined as the expectation of $\hat{\Delta}_{1}$ conditional on all the responses, $Y_{11}, Y_{12}, \cdots, Y_{2 n}$, and all the sets and ranks $R_{i j} ; i=1,2, \cdots, n, j=1,2, \cdots, n$, It can also be expressed as the average over all the possible pairings. Table 3.2 represents one possible pairing for the 8 EUs. It is easy to see that by rearranging the EUs, there are $4 !=24$ possible pairings in total. Since there are 24 possible pairings for this example, we can have 24 possible $\hat{\Delta}_{1}^{(l)}$ s $; l=1, \ldots, 24$, each with probability $\frac{1}{24}$. Consequently, $\hat{\Delta}_{2}$ can be derived by averaging these $24 \hat{\Delta}_{1}^{(l)}$ 's. Each $\hat{\Delta}_{1}^{(l)}$ can be computed as described in the previous paragraph. Then

$$
\hat{\Delta}_{2}=\frac{1}{24} \sum_{l=1}^{24} \hat{\Delta}_{1}^{(l)} .
$$

To obtain $\hat{\Delta}_{3}$, we randomly select one EU from the treatment group and another one from the control group to form a set. Then we have $4^{2}=16$ possible sets in total. Assume that all these 16 sets can be ranked as $\left\{u_{11}<u_{21} ; u_{11}<u_{22} ; u_{11}<\right.$ $\left.u_{23} ; u_{11}<u_{24}\right\},\left\{u_{12}<u_{21} ; u_{12}>u_{22} ; u_{12}<u_{23} ; u_{12}>u_{24}\right\},\left\{u_{13}>u_{21} ; u_{13}>\right.$ $\left.u_{22} ; u_{13}<u_{23} ; u_{13}>u_{24}\right\},\left\{u_{14}>u_{21} ; u_{14}>u_{22} ; u_{14}>u_{23} ; u_{14}>u_{24}\right\}$, where $u$ indicates the experimental unit. Then the VRBE $\hat{\Delta}_{3}$ is obtained by dividing all the $n^{2}$ sets into two different types of sets. In this example, seven pairs are assigned to Type 1 and the remaining ones are assigned to Type 2. Hence, $\hat{\Delta}_{3}=\frac{1}{2}\left(\hat{\delta}_{1}+\hat{\delta}_{2}\right)=$ $\frac{1}{2}\left\{\frac{1}{7}\left[\left(Y_{11}-Y_{21}\right)+\left(Y_{11}-Y_{22}\right)+\left(Y_{11}-Y_{23}\right)+\left(Y_{11}-Y_{24}\right)+\left(Y_{12}-Y_{21}\right)+\left(Y_{12}-Y_{23}\right)+\right.\right.$ $\left.\left(Y_{13}-Y_{23}\right)\right]+\frac{1}{9}\left[\left(Y_{12}-Y_{22}\right)+\left(Y_{12}-Y_{24}\right)+\left(Y_{13}-Y_{21}\right)+\left(Y_{13}-Y_{22}\right)+\left(Y_{13}-Y_{24}\right)+\right.$ $\left.\left.\left(Y_{14}-Y_{21}\right)+\left(Y_{14}-Y_{22}\right)+\left(Y_{14}-Y_{23}\right)+\left(Y_{14}-Y_{24}\right)\right]\right\}$. 


\subsection{Limiting Distribution of The VRBE}

We next derive the limiting distribution of VRBE. The main idea is to define VRBE as a generalized U-statistic and to utilize the asymptotic properties of generalized U-statistics to obtain its limiting distribution. We first define some new notation. Let $C_{1}, C_{2}, \cdots, C_{n}$ be $n$ two dimensional random vectors that are i.i.d. from the control population, i.e., $C_{i}=\left(Y_{1 i}, X_{1 i}\right)^{T} ; i=1,2, \cdots, n$, where $Y_{1 i}$ is the response and $X_{1 i}$ is a scalar which is implicitly used to rank the units. Similarly define $T_{1}, T_{2}, \cdots, T_{n}$ to be random vectors that are i.i.d. from the treatment population where $T_{j}=\left(Y_{2 j}, X_{2 j}\right)^{T} ; j=1,2, \cdots, n$. Then we can rewrite $\mathbf{W}$ as $U=\left[\frac{1}{n^{2}} \sum_{i=1}^{n} \sum_{j=1}^{n}\left(Y_{1 i}-Y_{2 j}\right) I\left(X_{1 i}<X_{2 j}\right), \frac{1}{n^{2}} \sum_{i=1}^{n} \sum_{j=1}^{n}\left(Y_{1 i}-Y_{2 j}\right) I\left(X_{1 i}>X_{2 j}\right)\right.$, $\left.\frac{1}{n^{2}} \sum_{i=1}^{n} \sum_{j=1}^{n} I\left(X_{1 i}<X_{2 j}\right), \frac{1}{n^{2}} \sum_{i=1}^{n} \sum_{j=1}^{n} I\left(X_{1 i}>X_{2 j}\right)\right]^{T}$. Note that the vector $\mathbf{W}$ is a four dimensional, two-sample generalized U-statistic with

$$
U^{(1)}=\frac{1}{n^{2}} \sum_{i=1}^{n} \sum_{j=1}^{n}\left(Y_{1 i}-Y_{2 j}\right) I\left(X_{1 i}<X_{2 j}\right)=\frac{1}{\left(\begin{array}{l}
n \\
1
\end{array}\right)\left(\begin{array}{l}
n \\
1
\end{array}\right)} \sum_{i=1}^{n} \sum_{j=1}^{n} \psi^{(1)}\left(C_{i}, T_{j}\right),
$$

where $\psi^{(1)}\left(C_{i}, T_{j}\right)=\left(Y_{1 i}-Y_{2 j}\right) I\left(X_{1 i}<X_{2 j}\right)$ is the kernel for $U^{(1)}$ and is symmetric in $C_{1}, \cdots, C_{n}$ and $T_{1}, \cdots, T_{n}$, with $\theta^{(1)}=E\left(\psi^{(1)}\left(C_{i}, T_{j}\right)\right)=\frac{1}{2} \delta_{1}$. Similarly,

$$
\begin{aligned}
U^{(2)} & =\frac{1}{n^{2}} \sum_{i=1}^{n} \sum_{j=1}^{n}\left(Y_{1 i}-Y_{2 j}\right) I\left(X_{1 i}>X_{2 j}\right)=\frac{1}{\left(\begin{array}{l}
n \\
1
\end{array}\right)\left(\begin{array}{l}
n \\
1
\end{array}\right)} \sum_{i=1}^{n} \sum_{j=1}^{n} \psi^{(2)}\left(C_{i}, T_{j}\right), \\
U^{(3)} & =\frac{1}{n^{2}} \sum_{i=1}^{n} \sum_{j=1}^{n} I\left(X_{1 i}<X_{2 j}\right)=\frac{1}{\left(\begin{array}{l}
n \\
1
\end{array}\right)\left(\begin{array}{l}
n \\
1
\end{array}\right)} \sum_{i=1}^{n} \sum_{j=1}^{n} \psi^{(3)}\left(C_{i}, T_{j}\right), \\
U^{(4)} & =\frac{1}{n^{2}} \sum_{i=1}^{n} \sum_{j=1}^{n} I\left(X_{1 i}>X_{2 j}\right)=\frac{1}{\left(\begin{array}{l}
n \\
1
\end{array}\right)\left(\begin{array}{l}
n \\
1
\end{array}\right)} \sum_{i=1}^{n} \sum_{j=1}^{n} \psi^{(4)}\left(C_{i}, T_{j}\right),
\end{aligned}
$$

where

$$
\psi^{(2)}\left(C_{i}, T_{j}\right)=\left(Y_{1 i}-Y_{2 j}\right) I\left(X_{1 i}>X_{2 j}\right),
$$


$\psi^{(3)}\left(C_{i}, T_{j}\right)=I\left(X_{1 i}<X_{2 j}\right)$, and $\psi^{(4)}\left(C_{i}, T_{j}\right)=I\left(X_{1 i}>X_{2 j}\right)$. These kernels are symmetric in $C_{1}, \cdots, C_{n}$ and $T_{1}, \cdots, T_{n}$, with $\theta^{(2)}=E\left(\psi^{(2)}\right)=\frac{1}{2} \delta_{2}, \theta^{(3)}=E\left(\psi^{(3)}\right)=$ $\frac{1}{2}$, and $\theta^{(4)}=E\left(\psi^{(4)}\right)=\frac{1}{2}$. We also define $\theta^{*}$ to be the expectation of $\mathrm{U}$ conditional on $m_{1}$, i.e. $\theta^{*}=\left(\theta^{*(1)}, \theta^{*(2)}, \theta^{*(3)}, \theta^{*(4)}\right)$, where $\theta^{*(1)}=E\left(\psi^{(1)}\left(C_{i}, T_{j}\right) \mid m_{1}\right)=\frac{m_{1}}{n^{2}} \delta_{1}, \theta^{*(2)}=$ $E\left(\psi^{(2)} \mid m_{1}\right)=\frac{n^{2}-m_{1}}{n^{2}} \delta_{2}, \theta^{*(3)}=E\left(\psi^{(3)} \mid m_{1}\right)=\frac{m_{1}}{n^{2}}$, and $\theta^{*(4)}=E\left(\psi^{(4)} \mid m_{1}\right)=\frac{n^{2}-m_{1}}{n^{2}}$. Then we have the following theorem:

Theorem 3 (Asymptotic Normality of $\hat{\Delta}_{3}$ ) Let $h_{i}^{(j)} ; i=1,2$, be the set of first kernel functions in the $H$ decomposition of $U^{(j)} ; j=1,2,3,4$. Then

$$
h_{i}^{(j)}(C)=E \psi^{(j)}\left(C, T_{j}\right)-\theta^{(j)} .
$$

Define $\Sigma_{1}$ and $\Sigma_{2}$ to be the variance matrices of the random vectors

$$
Z_{1, i}=\left(h_{1}^{(1)}\left(C_{i}\right), h_{1}^{(2)}\left(C_{i}\right), h_{1}^{(3)}\left(C_{i}\right), h_{1}^{(4)}\left(C_{i}\right)\right)
$$

and

$$
Z_{2, j}=\left(h_{2}^{(1)}\left(T_{j}\right), h_{2}^{(2)}\left(T_{j}\right), h_{2}^{(3)}\left(T_{j}\right), h_{2}^{(4)}\left(T_{j}\right)\right)
$$

respectively. Assume all the variances are finite. Then as $n \rightarrow \infty$, we have

$$
\frac{\sqrt{2 n}\left(\hat{\Delta}_{3}-\Delta\right)}{2 G^{\prime}\left(\theta^{*}\right)\left(\Sigma_{1}+\Sigma_{2}\right) G^{\prime T}\left(\theta^{*}\right)} \stackrel{D}{\rightarrow} N(0,1),
$$

where $G^{\prime}\left(\theta^{*}\right)$ is the first derivative of $G(x)=\frac{1}{2}\left(\frac{x_{1}}{x_{3}}+\frac{x_{2}}{x_{4}}\right)$, evaluated at

$$
\theta^{*}=\left(\theta^{*(1)}, \theta^{*(2)}, \theta^{*(3)}, \theta^{*(4)}\right)
$$

Proof of Theorem 3: For a balanced randomized two-sample design, we have equal sample sizes for the treatment and control. Let $n_{i}$ denote the sample size, we have $n_{1}=n_{2}=n$. As $n \rightarrow \infty, p_{i}=n_{i} / N=1 / 2$, where $N=n_{1}+n_{2}=2 n$. Also let $\theta=$ 
$\left(\theta^{(1)}, \theta^{(2)}, \theta^{(3)}, \theta^{(4)}\right)$ and $\theta^{*}=\left(\theta^{*(1)}, \theta^{*(2)}, \theta^{*(3)}, \theta^{*(4)}\right)$, where $\theta^{(j)}=E\left(\psi^{(j)}\left(C_{i}, T_{j}\right)\right)$ and $\theta^{*(j)}=E\left(\psi^{(j)}\left(C_{i}, T_{j}\right) \mid m_{1}\right)$. Then by properties of the multivariate normal distribution for generalized U-statistics (Lee 1990), we have $\sqrt{N}(U-\theta)$ converges in distribution to a multivariate normal distribution with mean vector zero and covariance matrix $\Sigma=p_{1}^{-1} k_{1}^{2} \Sigma_{1}+p_{2}^{-1} k_{2}^{2} \Sigma_{2}=2\left(\Sigma_{1}+\Sigma_{2}\right)$.

Let $G(x)=\frac{1}{2}\left(\frac{x_{1}}{x_{3}}+\frac{x_{2}}{x_{4}}\right)$, where $x=\left[x_{1}, x_{2}, x_{3}, x_{4}\right]^{T}$. If we apply $G(x)$ to the asymptotic distribution of the random vector $(U-\theta)$, the following result is readily obtained by the Delta method.

$$
\sqrt{N}(G(U)-G(\theta))=\sqrt{N}\left(\hat{\Delta}_{3}-\Delta\right) \underset{D}{D} N\left(0, G^{\prime}(\theta)\right) \Sigma G^{\prime T}(\theta),
$$

where $G^{\prime}(\theta)$ is the first derivative of $G(x)$ evaluated at $\theta$, i.e. $G^{\prime}(\theta)=\left(1,1,-\delta_{1},-\delta_{2}\right)$. Then we have

$$
\frac{\sqrt{N}\left(\hat{\Delta}_{3}-\Delta\right)}{G^{\prime}(\theta) \Sigma G^{\prime T}(\theta)} \underset{\rightarrow}{\rightarrow} N(0,1) .
$$

Applying the Slutsky's Theorem, we have

$$
\frac{\sqrt{2 n}\left(\hat{\Delta}_{3}-\Delta\right)}{2 G^{\prime}\left(\theta^{*}\right)\left(\Sigma_{1}+\Sigma_{2}\right) G^{\prime T}\left(\theta^{*}\right)} \stackrel{D}{\rightarrow} N(0,1),
$$

where $G^{\prime}\left(\theta^{*}\right)=\left(\frac{n^{2}}{2 m_{1}}, \frac{n^{2}}{2\left(n^{2}-m_{1}\right)},-\frac{n^{2}}{2 m_{1}} \delta_{1},-\frac{n^{2}}{2\left(n^{2}-m_{1}\right)} \delta_{2}\right)$.

\subsection{Inferential Procedures of the VRBE}

For the VRBE, the corresponding asymptotically normal, level $\alpha$ test follows from Theorem 3. For a two sided test,

$$
\left|Z_{n}^{3}\right|=\left\{\left|\sqrt{2 n} \hat{\Delta}_{3}\right| / \sqrt{2 G^{\prime}\left(\theta^{*}\right)\left(\Sigma_{1}+\Sigma_{2}\right) G^{\prime T}\left(\theta^{*}\right)}\right\} \geq u_{1-\alpha / 2}
$$

provides a hypothesis test with asymptotic level $\alpha$. 
According to the definition of $h_{i}^{(j)}$, we can derive the explicit form of $\Sigma_{1}$ and $\Sigma_{2}$, and hence, find the variance of limiting distribution $G^{\prime}(\theta) \Sigma G^{\prime T}$. First, define $\mu_{i[k: m]}, \sigma_{i[k: m]}^{2} ; i=1,2, k=1,2, \cdots, m$, as the mean and variance of the response in treatment $i$ with rank $k$ among $m$ units, $Y_{i[k: m]}$. Let $\sigma_{[1,2]}$ indicate the covariance of the units within a set. Then

$$
\Sigma_{1}=\left[\begin{array}{cccc}
\sigma_{1,0}^{2(1)} & \sigma_{1,0}^{(1,2)} & \sigma_{1,0}^{(1,3)} & \sigma_{1,0}^{(1,4)} \\
& \sigma_{1,0}^{2(2)} & \sigma_{1,0}^{(2,3)} & \sigma_{1,0}^{(2,4)} \\
& & \sigma_{1,0}^{2(3)} & \sigma_{1,0}^{(3,4)} \\
& & & \sigma_{1,0}^{2(4)}
\end{array}\right], \Sigma_{2}=\left[\begin{array}{cccc}
\sigma_{0,1}^{2(1)} & \sigma_{0,1}^{(1,2)} & \sigma_{0,1}^{(1,3)} & \sigma_{0,1}^{(1,4)} \\
& \sigma_{0,1}^{2(2)} & \sigma_{0,1}^{(2,3)} & \sigma_{0,1}^{(2,4)} \\
& & \sigma_{0,1}^{2(3)} & \sigma_{0,1}^{(3,4)} \\
& & & \sigma_{0,1}^{2(4)}
\end{array}\right],
$$

where $\sigma_{1,0}^{2(j)}=\operatorname{Cov}\left(\psi^{(j)}\left(C_{i}, T_{j}\right), \psi^{(j)}\left(C_{i}, T_{l}\right)\right), \sigma_{1,0}^{(i, j)}=\operatorname{Cov}\left(\psi^{(i)}\left(C_{i}, T_{j}\right), \psi^{(j)}\left(C_{i}, T_{l}\right)\right)$, $\sigma_{0,1}^{2(j)}=\operatorname{Cov}\left(\psi^{(j)}\left(C_{i}, T_{j}\right), \psi^{(j)}\left(C_{l}, T_{j}\right)\right)$, and $\sigma_{0,1}^{(i, j)}=\operatorname{Cov}\left(\psi^{(i)}\left(C_{i}, T_{j}\right), \psi^{(j)}\left(C_{l}, T_{j}\right)\right)$. Note that by the symmetric property of a covariance matrix, we only display the upper right parts of $\Sigma_{1}$ and $\Sigma_{2}$. By direct calculation, we have for $\Sigma_{1}$

$$
\begin{gathered}
\sigma_{1,0}^{2(1)}=\frac{1}{3}\left[\sigma_{1[1: 3]}^{2}-2 \sigma_{[1,2]}+\left(\mu_{1[1: 3]}-\mu_{2[2: 2]}\right)^{2}\right]-\frac{1}{4}\left[\left(\mu_{1[1: 2]}\right)-\left(\mu_{2[2: 2]}\right)\right]^{2}, \\
\sigma_{1,0}^{2(2)}=\frac{1}{3}\left[\sigma_{1[3: 3]}^{2}-2 \sigma_{[1,2]}+\left(\mu_{1[3: 3]}-\mu_{2[1: 2]}\right)^{2}\right]-\frac{1}{4}\left[\left(\mu_{1[2: 2]}\right)-\left(\mu_{2[1: 2]}\right)\right]^{2}, \\
\sigma_{1,0}^{2(3)}=\sigma_{1,0}^{2(4)}=\frac{1}{12}, \\
\sigma_{1,0}^{(1,2)}=\frac{1}{6}\left[\sigma_{1[2: 3]}^{2}+\mu_{1[2: 3]}^{2}-2 \sigma_{[1,2]}-\left(\mu_{1[2: 3]} \mu_{2[1: 2]}\right)\right. \\
\left.-\left(\mu_{1[2: 3]} \mu_{2[2: 2]}\right)+\left(\mu_{2[1: 2]} \mu_{2[2: 2]}\right)\right] \\
-\frac{1}{4}\left(\mu_{1[1: 2]}-\mu_{2[2: 2]}\right)\left(\mu_{1[2: 2]}-\mu_{2[1: 2]}\right), \\
\sigma_{1,0}^{(1,3)=}=\frac{1}{12}\left(\mu_{1[1: 2]}-\mu_{2[2: 2]}\right), \\
\sigma_{1,0}^{(1,4)}=-\frac{1}{12}\left(\mu_{1[1: 2]}-\mu_{2[2: 2]}\right), \sigma_{1,0}^{(2,3)}=-\frac{1}{12}\left(\mu_{1[2: 2]}-\mu_{2[1: 2]}\right), \\
\sigma_{1,0}^{(2,4)}=\frac{1}{12}\left(\mu_{1[2: 2]}-\mu_{2[1: 2]}\right), \sigma_{1,0}^{(3,4)}=-\frac{1}{12} .
\end{gathered}
$$

Similarly, we get the following results for $\Sigma_{2}$ :

$$
\sigma_{0,1}^{2(1)}=\frac{1}{3}\left[\sigma_{2[3: 3]}^{2}-2 \sigma_{[1,2]}+\left(\mu_{2[3: 3]}-\mu_{1[1: 2]}\right)^{2}\right]-\frac{1}{4}\left[\left(\mu_{1[1: 2]}\right)-\left(\mu_{2[2: 2]}\right)\right]^{2},
$$




$$
\begin{gathered}
\sigma_{0,1}^{2(2)}=\frac{1}{3}\left[\sigma_{2[1: 3]}^{2}-2 \sigma_{[1,2]}+\left(\mu_{2[1: 3]}-\mu_{1[2: 2]}\right)^{2}\right]-\frac{1}{4}\left[\left(\mu_{1[2: 2]}\right)-\left(\mu_{2[1: 2]}\right)\right]^{2}, \\
\sigma_{0,1}^{2(3)}=\sigma_{0,1}^{2(4)}=\frac{1}{12}, \\
\sigma_{0,1}^{(1,2)}=\frac{1}{6}\left[\sigma_{2[2: 3]}^{2}+\mu_{2[2: 3]}^{2}-2 \sigma_{[1,2]}-\left(\mu_{2[2: 3]} \mu_{2[1: 2]}\right)\right. \\
\left.-\quad\left(\mu_{2[2: 3]} \mu_{1[2: 2]}\right)+\left(\mu_{1[1: 2]} \mu_{1[2: 2]}\right)\right] \\
-\frac{1}{4}\left(\mu_{1[1: 2]}-\mu_{2[2: 2]}\right)\left(\mu_{1[2: 2]}-\mu_{2[1: 2]}\right), \\
\sigma_{0,1}^{(1,3)}=\sigma_{1,0}^{(1,3)}, \sigma_{0,1}^{(1,4)}=\sigma_{1,0}^{(1,4)}, \sigma_{0,1}^{(2,3)}=\sigma_{1,0}^{(2,3)}, \sigma_{0,1}^{(2,4)}=\sigma_{1,0}^{(2,4)}, \sigma_{0,1}^{(3,4)}=\sigma_{1,0}^{(3,4)} .
\end{gathered}
$$

Then the variance of the limiting distribution is

$$
\begin{aligned}
\sigma^{2} & =G^{\prime}(\theta) \Sigma G^{\prime} T(\theta) \\
& =2\left[\left(\sigma_{1,0}^{2(1)}+\sigma_{0,1}^{2(1)}\right)+2\left(\sigma_{1,0}^{(1,2)}+\sigma_{0,1}^{(1,2)}\right)+\left(\sigma_{1,0}^{2(2)}+\sigma_{0,1}^{2(2)}\right)\right] \\
& -\frac{1}{3}\left(\mu_{1[1: 2]}-\mu_{2[2: 2]}\right)\left(\mu_{1[2: 2]}-\mu_{2[1: 2]}\right) .
\end{aligned}
$$

To estimate the variance for the limiting distribution, two methods are considered. One is to estimate the parameters involved in the final form of the variance, since all of them can be written as the means and variances for the responses. Simulation shows that, however, this method tends to overestimate the variance and consequently delivers bad performance. The other method is to estimate $\Sigma_{1}$ and $\Sigma_{2}$ directly by obtaining two sets of samples for the four dimensional random vectors $Z_{1, i}$ and $Z_{2, j}$. Let $Z_{1, i}^{*}=\left(h_{1}^{(* 1)}\left(C_{i}\right), h_{1}^{(* 2)}\left(C_{i}\right), h_{1}^{(* 3)}\left(C_{i}\right), h_{1}^{(* 4)}\left(C_{i}\right)\right)$ and $Z_{2, j}^{*}=\left(h_{2}^{(* 1)}\left(T_{j}\right), h_{2}^{(* 2)}\left(T_{j}\right), h_{2}^{(* 3)}\left(T_{j}\right), h_{2}^{(* 4)}\left(T_{j}\right)\right) ; i=1,2, \cdots, n, j=1,2, \cdots, n$, denote the samples. According to the definition of $h_{i}^{(j)}(C)$, the sample $h_{i}^{(* j)}(C)$ can be obtained by averaging the $j$-th kernel over $T_{j} ; j=1,2, \cdots, n$, i.e. $h_{i}^{(* j)}(C)=$ $\frac{1}{n} \sum_{j=1}^{n} \psi^{(j)}\left(C, T_{j}\right)$. Therefore the sample variance-covariance matrices are consistent estimators for $\Sigma_{1}$ and $\Sigma_{2}$. If we plug in the estimate of the variance for the limiting 
distribution, we get an asymptotic normal test. As before, the $t$-distribution with $n-1$ d.f. provides a better approximation than does the normal for small or moderate sample sizes. Interval estimations for the contrast parameter can be obtained by inverting the hypothesis test.

\subsection{An Illustration with a Two-Sample T-Test}

For estimating the variance of $\hat{\Delta}_{3}$, there are two reasonable choices: $G^{\prime}(\theta) \mathbf{S} G^{\prime}(\theta)$ and $G^{\prime}\left(\theta^{*}\right) \mathbf{S} G^{\prime T}\left(\theta^{*}\right)$, where $\mathbf{S}$ is the sample variance-covariance matrix for $\Sigma$. Following the proof of Theorem 3, we know that $G^{\prime}(\theta) \mathbf{S} G^{\prime T}(\theta)$ provides an estimate of the variance of the limiting normal distribution for $\hat{\Delta}_{3}$. It is a good estimator of the limiting variance of $\hat{\Delta}_{3}$ for large sample sizes, but tends to underestimate the actual variance for small samples. Hence, we use $G^{\prime}\left(\theta^{*}\right) \mathbf{S} G^{\prime} T\left(\theta^{*}\right)$ as the estimator of the variance to derive the inferential procedures for $\hat{\Delta}_{3}$.

To further illustrate the idea via which we obtain the VRBE and the corresponding hypothesis testing procedure, we derive the two-sample $t$-test through the same approach as described above. Recall that for the VRBE, all the $n^{2}$ sets are ranked and assigned as Type 1 or Type 2 sets. Then the number of Type 1 sets, $m_{1}$, converges to $n^{2} / 2$ as $n \rightarrow \infty$. Similarly, for the two-sample $t$-test we assume that there are $n$ EUs and each will be assigned to the control or treatment by flipping a fair coin. Let $R_{i} ; i=1,2, \cdots, n$, indicate the result of the assignment, $r_{1}$ represent the number of units assigned to the control, and let $S_{r}$ be the collection of these units, so that

$$
R_{i}= \begin{cases}1 & \text { when } Y_{i} \in S_{r} \\ 0 & \text { when } Y_{i} \notin S_{r}\end{cases}
$$


Define a four-dimensional random vector $\mathbf{V}_{i}=\left[Y_{i}, 0,1,0\right]^{T}$ if $R_{i}=1$ and $\left[0, Y_{i}, 0,1\right]^{T}$ otherwise. Then the average of these $\mathbf{V}_{i}$ 's can be expressed as

$$
\mathbf{V}_{n}=\left[\frac{r_{1}}{n} \frac{1}{r_{1}} \sum_{Y_{i} \in S_{r}} Y_{i}, \frac{n-r_{1}}{n} \frac{1}{n-r_{1}} \sum_{Y_{i} \notin S_{r}} Y_{i}, \frac{r_{1}}{n}, \frac{n-r_{1}}{n}\right]^{T} .
$$

Define $\psi_{1}=E\left(Y_{i} \mid R_{i}=1\right)$ and $\psi_{2}=E\left(Y_{i} \mid R_{i}=0\right)$. The expectation of $\mathbf{V}_{n}$, conditional on $r_{1}$ is

$$
E\left(\mathbf{V}_{n} \mid r_{1}\right)=\left[\frac{r_{1}}{n} \psi_{1}, \frac{n-r_{1}}{n} \psi_{2}, \frac{r_{1}}{n}, \frac{n-r_{1}}{n}\right]^{T}
$$

We are interested in estimating $\psi_{1}-\psi_{2}$. Denote $F(x)$ as $\left(\frac{x_{1}}{x_{3}}-\frac{x_{2}}{x_{4}}\right)$, where $x=$ $\left[x_{1}, x_{2}, x_{3}, x_{4}\right]^{T}, F\left(\mathbf{V}_{n}\right)=\frac{1}{r_{1}} \sum_{Y_{i} \in S_{r}} Y_{i}-\frac{1}{n-r_{1}} \sum_{Y_{i} \notin S_{r}} Y_{i}=\bar{Y}_{1 .}-\bar{Y}_{2 .}$. A Taylor series expansion yields $F(x)=F\left(x_{0}\right)+F^{\prime}\left(x_{0}\right)\left(x-x_{0}\right)+o\left(x-x_{0}\right)$, where $F^{\prime}\left(x_{0}\right)$ is the first order derivative evaluated at $x_{0}$. Replacing $x$ and $x_{0}$ by $\mathbf{V}_{n}$ and $E\left(\mathbf{V}_{n} \mid r_{1}\right)$, respectively, we then have

$$
\begin{aligned}
F(x) & =\frac{1}{r_{1}} \sum_{Y_{i} \in S_{r}} Y_{i}-\frac{1}{n-r_{1}} \sum_{Y_{i} \notin S_{r}} Y_{i}=\bar{Y}_{1 .}-\bar{Y}_{2 .}, \\
F\left(x_{0}\right) & =\psi_{1}-\psi_{2}, \\
F^{\prime}\left(x_{0}\right) & =\left[\frac{1}{r_{1} / n},-\frac{1}{\left(n-r_{1}\right) / n},-\frac{\psi_{1}}{r_{1} / n}, \frac{\psi_{2}}{\left(n-r_{1}\right) / n}\right] .
\end{aligned}
$$

It is clear that $F\left(\mathbf{V}_{n}\right)=\bar{Y}_{1} .-\bar{Y}_{2}$. is an asymptotically unbiased estimator of $F\left(E\left(\mathbf{V}_{n} \mid r_{1}\right)\right)=\psi_{1}-\psi_{2}$, with variance matrix $F^{\prime}\left(E\left(\mathbf{V}_{n} \mid r_{1}\right)\right) \Sigma_{r} F^{\prime T}\left(E\left(\mathbf{V}_{n} \mid r_{1}\right)\right)$, where $n \operatorname{Var}\left(\mathbf{V}_{n}\right)=\Sigma_{r}$. We replace $\psi_{i}$ by the sample means $\bar{Y}_{i .} ; i=1,2$, and $\Sigma_{r}$ by the sample variance matrix $\mathbf{S}_{r}$, where

$$
\mathbf{S}_{r}=\left[\begin{array}{cccc}
\frac{r_{1}}{n} S_{1}^{2}+\frac{r_{1}\left(n-r_{1}\right)}{Y_{1}^{2}} \bar{Y}_{1 .} & -\frac{r_{1}\left(n-r_{1}\right)}{n^{2}} \bar{Y}_{1 .} \bar{Y}_{2 .} & \frac{r_{1}\left(n-r_{1}\right)}{n^{2}} \bar{Y}_{1 .} & -\frac{r_{1}\left(n-r_{1}\right)}{n^{2}} \bar{Y}_{1 .} \\
-\frac{r_{1}\left(n-r_{1}\right)}{n^{2}} \bar{Y}_{1 .} \bar{Y}_{2 .} & \frac{n-r_{1}}{n} S_{2}^{2}+\frac{r_{1}\left(n-r_{1}\right)}{n_{Y}} \bar{Y}_{2 .}^{2} & -\frac{r_{1}\left(n-r_{1}\right)}{n^{2}} \bar{Y}_{2 .} & \frac{r_{1}\left(n-r_{1}\right)}{n^{2}} \bar{Y}_{2 .} . \\
\frac{r_{1}\left(n-r_{1}\right)}{n^{2}} \bar{Y}_{1 .} & -\frac{r_{1}\left(n-r_{1}\right)}{n^{2}} \bar{Y}_{2 .} . & \frac{r_{1}\left(n-r_{1}\right)}{n^{2}} & -\frac{r_{1}\left(n-r_{1}\right)}{n^{2}} \\
-\frac{r_{1}\left(n-r_{1}\right)}{n^{2}} \bar{Y}_{1 .} & \frac{r_{1}\left(n-r_{1}\right)}{n^{2}} \bar{Y}_{2} . & -\frac{r_{1}\left(n-r_{1}\right)}{n^{2}} & \frac{r_{1}\left(n-r_{1}\right)}{n^{2}}
\end{array}\right] .
$$

Then the estimated variance for $\bar{Y}_{1}-\bar{Y}_{2}$. is

$$
\frac{1}{n} \hat{F}^{\prime}\left(E\left(\mathbf{V}_{n} \mid r_{1}\right)\right) \mathbf{S}_{r} \hat{F}^{\prime T}\left(E\left(\mathbf{V}_{n} \mid r_{1}\right)\right)=\frac{S_{1}^{2}}{r_{1}}+\frac{S_{2}^{2}}{n-r_{1}}
$$


which is same as the variance estimation for a two-sample $t$-test.

The limiting distribution of $\bar{Y}_{1}$. $-\bar{Y}_{2}$. can be obtained by applying the Central Limit Theorem followed by the Delta Method, hence, we have another reasonable estimator for the variance. Recall that $\mathbf{V}_{n}$ is the average of the $\mathbf{V}_{i}$ 's. The expectation of $\mathbf{V}_{n}$ is

$$
E\left(\mathbf{V}_{n}\right)=\left[\frac{1}{2} \alpha_{1}, \frac{1}{2} \alpha_{2}, \frac{1}{2}, \frac{1}{2}\right]^{T}
$$

Since the $\mathbf{V}_{i}$ are independent and identically distributed, by the standard Central Limit Theorem, we have

$$
\sqrt{n}\left(\mathbf{V}_{n}-E\left(\mathbf{V}_{n}\right)\right) \underset{D}{D} N\left(0, \Sigma_{r}\right)
$$

Apply $F(x)=\left(\frac{x_{1}}{x_{3}}-\frac{x_{2}}{x_{4}}\right)$, where $x=\left[x_{1}, x_{2}, x_{3}, x_{4}\right]^{T}$, on the above result. Then the following result is readily obtained:

$$
\begin{aligned}
& \sqrt{n}\left(F\left(\mathbf{V}_{n}\right)-F\left(E\left(\mathbf{V}_{n}\right)\right)\right)= \\
& \sqrt{n}\left[\left(\bar{Y}_{1 .}-\bar{Y}_{2 .}\right)-\left(\alpha_{1}-\alpha_{2}\right)\right] \underset{D}{\longrightarrow} N\left(0, F^{\prime}\left(E\left(\mathbf{V}_{n}\right)\right) \Sigma_{r} F^{\prime T}\left(E\left(\mathbf{V}_{n}\right)\right)\right),
\end{aligned}
$$

where $F^{\prime}\left(E\left(\mathbf{V}_{n}\right)\right)$ is the first order derivative evaluated at $E\left(\mathbf{V}_{n}\right)$.

A reasonable estimator for the limiting variance is obtained by replacing $\alpha_{i}$ by the sample mean $\bar{Y}_{i .} ; i=1,2$, and $\Sigma_{r}$ by the corresponding sample variance matrix $\mathbf{S}_{r}$ for $F^{\prime}\left(E\left(\mathbf{V}_{n}\right)\right) \Sigma_{r} F^{\prime} T\left(E\left(\mathbf{V}_{n}\right)\right)$. Then we have

$$
\hat{F}^{\prime}\left(E\left(\mathbf{V}_{n}\right)\right) \mathbf{S}_{r} \hat{F}^{\prime T}\left(E\left(\mathbf{V}_{n}\right)\right)=4 \frac{r_{1}}{n} S_{1}^{2}+4 \frac{\left(n-r_{1}\right)}{n} S_{2}^{2}
$$

Hence another estimator for the variance of $\bar{Y}_{1}-\bar{Y}_{2}$ is

$$
\frac{1}{n} \hat{F}^{\prime}\left(E\left(\mathbf{V}_{n}\right)\right) \mathbf{S}_{r} \hat{F}^{\prime T}\left(E\left(\mathbf{V}_{n}\right)\right)=4 \frac{r_{1}^{2}}{n^{2}} \frac{S_{1}^{2}}{r_{1}}+4 \frac{\left(n-r_{1}\right)^{2}}{n^{2}} \frac{S_{2}^{2}}{n-r_{1}}
$$


We notice that $r_{1}$ converges to $n / 2$, therefore, this variance estimator is asymptotically

equivalent to $\frac{S_{1}^{2}}{r_{1}}+\frac{S_{2}^{2}}{n-r_{1}}$. For small sample size, however, $\frac{S_{1}^{2}}{r_{1}}+\frac{S_{2}^{2}}{n-r_{1}}$ is preferred. Applying Slutsky's Theorem, we have

$$
\frac{\sqrt{n}\left[\left(\bar{Y}_{1 .}-\bar{Y}_{2 .}\right)-\left(\alpha_{1}-\alpha_{2}\right)\right]}{F^{\prime}\left(E\left(\mathbf{V}_{n} \mid r_{1}\right)\right) \Sigma_{r} F^{\prime T}\left(E\left(\mathbf{V}_{n} \mid r_{1}\right)\right)} \stackrel{D}{\rightarrow} N(0,1)
$$

Therefore, in this case, we use $\frac{S_{1}^{2}}{r_{1}}+\frac{S_{2}^{2}}{n-r_{1}}$ as the variance estimator for the two-sample $t$-test. Using the same argument as for two-sample $t$-test, we choose $G^{\prime}\left(\theta^{*}\right) \mathbf{S} G^{\prime T}\left(\theta^{*}\right)$ as the variance estimator for the $\operatorname{VRBE} \hat{\Delta}_{3}$.

\subsection{The Example in Section 3.1.3 Continued}

We now return to our example in Section 3.1.3 for illustration of estimating the variances for $\hat{\Delta}_{1}$ and $\hat{\Delta}_{3}$, and obtaining the test statistics. Recall that in our example 4 sets are randomly formed and ranked. The JPS $\hat{\Delta}_{1}$ is calculated based on pairing the ranking results as listed in Table 3.2. Since the variances $\sigma_{X}^{2}$ and $\sigma_{Z}^{2}$ are unknown, we assume equal variances for the two groups and use the pooled estimator $S_{p}^{2}=$ $\frac{1}{n-2}\left[\left(n_{1}-1\right) S_{X}^{2}+\left(n-n_{1}-1\right) S_{Z}^{2}\right]$. For our example we have $n_{1}=n-n_{1}=2$, $\bar{X}=\frac{1}{2}\left[\left(Y_{11}-Y_{21}\right)+\left(Y_{13}-Y_{23}\right)\right]$ and $\bar{Z}=\frac{1}{2}\left[\left(Y_{12}-Y_{22}\right)+\left(Y_{14}-Y_{24}\right)\right]$. Then $S_{X}^{2}=\left(\left(Y_{11}-Y_{21}\right)-\bar{X}\right)^{2}+\left(\left(Y_{13}-Y_{23}\right)-\bar{X}\right)^{2}, S_{Z}^{2}=\left(\left(Y_{12}-Y_{22}\right)-\bar{Z}\right)^{2}+\left(\left(Y_{14}-Y_{24}\right)-\bar{Z}\right)^{2}$, and $S_{p}^{2}=\frac{1}{2}\left[S_{X}^{2}+S_{Z}^{2}\right]$. Hence, the variance of $\hat{\Delta}_{1}$ can be estimated by

$$
\begin{aligned}
\widehat{\operatorname{Var}}\left(\hat{\Delta}_{1}\right) & =\sqrt{\frac{1}{4}\left(\frac{1}{2}+\frac{1}{2}\right) S_{p}^{2}} \\
& =\frac{1}{2} S_{p} .
\end{aligned}
$$

To compute the variance estimator for $\hat{\Delta}_{3}$, we first compute the $4 \times 4$ difference matrix $D_{y}$ between the responses for the treatment and control groups, where the $i j$ th element of $D_{y}$ is $y_{1 i}-y_{2 j} ; i, j=1,2,3,4$. Note that the rows of $D_{y}$ correspond to 
the control group while the columns represent the treatment group. Then we compute the $4 \times 4$ ranking matrix $R_{x}$ with the $i j$-th element being $I\left(x_{1 i}<x_{2 j}\right)$. Similar to $D_{y}$, the rows of $R_{x}$ correspond to the control group while the columns represent the treatment group. In this case, we have

$$
R_{x}=\left[\begin{array}{llll}
1 & 1 & 1 & 1 \\
1 & 0 & 1 & 0 \\
0 & 0 & 1 & 0 \\
0 & 0 & 0 & 0
\end{array}\right]
$$

We then compute two $4 \times 4$ matrices $M_{x y}^{1}$ and $M_{x y}^{2}$, where $M_{x y}^{1}$ and $M_{x y}^{2}$ are defined as the elementwise products between $D_{y}$ and $R_{x}$ and between $D_{y}$ and $J-R_{x}$ with $J$ as a matrix of 1's, respectively. Then $h_{1}^{* 1}\left(C_{i}\right)$ can be calculated as the row average of the $i$-th row of $M_{x y}^{1}, h_{1}^{* 2}\left(C_{i}\right)$ is the row average of the $i$-th row of $M_{x y}^{2}, h_{1}^{* 3}\left(C_{i}\right)$ is the $i$-th row average of $R_{x}$, and $h_{1}^{* 4}\left(C_{i}\right)$ is the $i$-th row average of $J-R_{x}$, where $i=1,2,3,4$. Therefore, $\left(Z_{1,1}^{*}, Z_{1,2}^{*}, Z_{1,3}^{*}, Z_{1,4}^{*}\right)^{T}$ can be summarized as

$$
\left[\begin{array}{cccc}
\frac{1}{4}\left(4 y_{11}-y_{21}-y_{22}-y_{23}-y_{24}\right) & 0 & 1 & 0 \\
\frac{1}{4}\left(2 y_{12}-y_{21}-y_{23}\right) & \frac{1}{4}\left(2 y_{12}-y_{22}-y_{24}\right) & \frac{1}{2} & \frac{1}{2} \\
\frac{1}{4}\left(y_{13}-y_{23}\right) & \frac{1}{4}\left(3 y_{13}-y_{21}-y_{22}-y_{24}\right) & \frac{1}{4} & \frac{3}{4} \\
0 & \frac{1}{4}\left(4 y_{14}-y_{21}-y_{22}-y_{23}-y_{24}\right) & 0 & 1
\end{array}\right]
$$

Let $\bar{Z}_{1}^{*}=\frac{1}{4}\left(Z_{1,1}^{*}+Z_{1,2}^{*}+Z_{1,3}^{*}+Z_{1,4}^{*}\right)$ and $Z_{1}^{*}=\left(Z_{1,1}^{*}-\bar{Z}_{1}^{*}, Z_{1,2}^{*}-\bar{Z}_{1}^{*}, Z_{1,3}^{*}-\bar{Z}_{1}^{*}, Z_{1,4}^{*}-\bar{Z}_{1}^{*}\right)^{T}$. Then $\Sigma_{1}$ can be estimated by $S_{1}=\frac{1}{n-1} Z_{1}^{* T} Z_{1}^{*}$.

Similarly, $h_{1}^{* 1}\left(T_{i}\right)$ can be calculated as the column average of the $i$-th column of $M_{x y}^{1}, h_{1}^{* 2}\left(T_{i}\right)$ is the column average of the $i$-th column of $M_{x y}^{2}, h_{1}^{* 3}\left(T_{i}\right)$ is the $i$ th column average of $R_{x}$, and $h_{1}^{* 4}\left(T_{i}\right)$ is the $i$-th column average of $J-R_{x}$, where $i=1,2,3,4$. Then $Z_{2}^{*}=\left(Z_{2,1}^{*}, Z_{2,2}^{*}, Z_{2,3}^{*}, Z_{2,4}^{*}\right)^{T}$ can be summarized as

$$
\left[\begin{array}{cccc}
\frac{1}{4}\left(y_{11}+y_{12}-2 y_{21}\right) & \frac{1}{4}\left(y_{13}+y_{14}-2 y_{21}\right) & \frac{1}{2} & \frac{1}{2} \\
\frac{1}{4}\left(y_{11}-y_{22}\right) & \frac{1}{4}\left(y_{12}+y_{13}+y_{14}-3 y_{22}\right) & \frac{1}{4} & \frac{3}{4} \\
\frac{1}{4}\left(y_{11}+y_{12}+y_{13}-3 y_{23}\right) & \frac{1}{4}\left(y_{14}-y_{23}\right) & \frac{3}{4} & \frac{1}{4} \\
\frac{1}{4}\left(y_{14}-y_{24}\right) & \frac{1}{4}\left(y_{12}+y_{13}+y_{14}-3 y_{24}\right) & \frac{1}{4} & \frac{3}{4}
\end{array}\right]
$$


Let $\bar{Z}_{2}^{*}=\frac{1}{4}\left(Z_{2,1}^{*}+Z_{2,2}^{*}+Z_{2,3}^{*}+Z_{2,4}^{*}\right)$ and $Z_{2}^{*}=\left(Z_{2,1}^{*}-\bar{Z}_{2}^{*}, Z_{2,2}^{*}-\bar{Z}_{2}^{*}, Z_{2,3}^{*}-\bar{Z}_{2}^{*}, Z_{2,4}^{*}-\bar{Z}_{2}^{*}\right)^{T}$. Then $\Sigma_{2}$ can be estimated by $S_{2}=\frac{1}{n-1} Z_{2}^{* T} Z_{2}^{*}$.

Using the results of Theorem 4 , we can get $\hat{G}^{\prime}\left(\theta^{*}\right)=\left(\frac{16}{14}, \frac{16}{18},-\frac{16}{14} \hat{\delta}_{1},-\frac{16}{18} \hat{\delta}_{2}\right)$. Thus, the variance of $\hat{\Delta}_{3}$ can be estimated by $\widehat{\operatorname{Var}}\left(\hat{\Delta}_{3}\right)=2 \hat{G}^{\prime}\left(\theta^{*}\right)\left(S_{1}+S_{2}\right) \hat{G}^{\prime}\left(\theta^{*}\right)^{T}$.

\subsection{Simulations}

We examine the performance of the VRBE $\hat{\Delta}_{3}$ and the corresponding testing procedures via a simulation study. First, the properties of the VRBE $\hat{\Delta}_{3}$ are investigated graphically based on 10,000 replicates and compared with those $\hat{\Delta}_{1}$ and the SRS estimator $\hat{\Delta}$. The true difference of treatment effects $\Delta=\mu_{1}-\mu_{2}$ is taken to be -1 . All scale parameters are set to 1 . Figure 3.1 shows the normal probability plots for the new estimators $\hat{\Delta}_{1}, \hat{\Delta}_{3}$, and $\hat{\Delta}$ with $n=10$ under perfect rankings. As discussed in the previous chapter, compared to the SRS estimator, $\hat{\Delta}_{1}$ has a slightly flatter slope and heavy tails on both sides. The flatter slope indicates less variation of the estimator, while the outliers on both sides correspond to the small or large values for $n_{1}$. The estimator $\hat{\Delta}_{3}$ has less variation than either $\hat{\Delta}$ or $\hat{\Delta}_{1}$. The linearity in the plot shows that $\hat{\Delta}_{3}$ is nearly normally distributed even for such a small $\mathrm{n}$.

Table 3.1 confirms that $\hat{\Delta}_{1}, \hat{\Delta}_{2}$, and $\hat{\Delta}_{3}$ have smaller mean square errors than $\hat{\Delta}$ when rankings are of reasonable quality. The MSEs for the new estimators are much smaller than that for $\hat{\Delta}$ when $\rho=0.7$ or 1 . They are slightly higher when $\rho=0$.

Table 3.3 reports the estimated levels of a nominal $\alpha=0.05$ test for the VRBE $\hat{\Delta}_{3}$, including limiting normal distribution $\left(Z_{n}^{3}\right)$ and Student's $t$-distribution $\left(T_{n}^{3}\right)$ with $n-1$ d.f. based on 10,000 replicates. We compare them with the other five types of testing procedures proposed in the previous chapter, three of which, including 
Student's $t$-distribution $\left(T_{n}\right)$ with $n-2$ d.f., the limiting normal distribution $\left(Z_{n}\right)$, and the Satterthwaite's approximation $\left(S_{n}\right)$, are for the JPS estimator $\hat{\Delta}_{1}$. The remaining two are for the SRS estimator $\hat{\Delta}$, i.e., Student's $t$-distribution $\left(T_{n}^{S R S}\right)$ with $2 n-2$ d.f. and the limiting normal distribution $\left(Z_{n}^{S R S}\right)$. As before $\sigma_{1}$ and $\sigma_{2}$ are set to 1 and the hypothesis test is $H_{0}: \mu_{1}=\mu_{2}$ versus $H_{a}: \mu_{1} \neq \mu_{2}$. Results show that $T_{n}^{3}$ is a conservative test compared with $Z_{n}^{3}$. The estimated levels for $Z_{n}^{3}$ exceed 0.05 for the small sample size $n=10$. As the sample size gets larger, however, the estimated levels for $Z_{n}^{3}$ get closer to the nominal level. Compared to the normal approximation, the $t$-distribution provides a better reference distribution.

We also simulate the empirical powers for $Z_{n}^{3}, T_{n}^{3}, T_{n}$, and $T_{n}^{S R S}$. Figure 3.2 shows that the tests $Z_{n}^{3}$ and $T_{n}^{3}$ based on $\hat{\Delta}_{3}$, and $T_{n}$ based on $\hat{\Delta}_{1}$, outperform the two sample $t$-test for the SRS estimator $T_{n}^{S R S}$ with a good ranking quality ranging from $\rho=1$ to 0.5. $Z_{n}^{3}$ based on $\hat{\Delta}_{3}$ delivers the best performance among them. The difference in performance gets smaller as the sample size becomes larger and the ranking quality decreases.

The last part of the simulation study focuses on examining the robustness of $\hat{\Delta}_{3}$. We first calculate the estimated levels for a nominal level $\alpha=0.05$ test based on 10,000 replicates when treatment variances are set to 1 and 4 . We compare the seven test statistic in Table 3.4. We find that the levels become large compared to the levels under equal variance assumption, while the $t$-test for $\hat{\Delta}_{1}$ and $\hat{\Delta}_{3}$ show consistent performance. The normal approximation requires larger sample sizes to get close to the nominal level. We also compare the empirical powers of $T_{n}$ and $Z_{n}$ for $\hat{\Delta}_{1}, T_{n}^{S R S}$, and $Z_{n}^{S R S}$ for $\hat{\Delta}, T_{n}^{3}$ and $Z_{n}^{3}$ for $\hat{\Delta}_{3}$. All the test statistics for $\hat{\Delta}_{1}$ and $\hat{\Delta}_{3}$ outperform that for the SRS estimator $\hat{\Delta}$. The test based on limiting normal distribution for $\hat{\Delta}_{3}$ 
is most powerful. For each estimator, the normal approximation has higher power than the pooled $t$-test.

We also study the performance of the VRBE $\hat{\Delta}_{3}$ when the underlying distribution of error term $\epsilon$ is actually skewed or has thicker tail under the perfect ranking situation. As before, we first use lognormal as the true distribution and the shape parameter $\sigma$ to control the skewness. As the values of $\sigma$ get larger, the lognormal distribution becomes more skewed. In this study, the mean of $\log (\epsilon)$ is 0 and standard deviations of $\log (\epsilon)$ are set to $0.5,1,2$ and 4. The results are presented in Table 3.6. It is well demonstrated that when the skewness increases all levels become smaller. While for relatively small sample sizes the test levels for $\hat{\Delta}_{1}$ and $\hat{\Delta}_{3}$ decrease slowly than that for the SRS estimator. Table 3.5 presents the estimated levels when the underlying distribution follows $t$-distribution and the d.f. are chosen to be $5,10,20$, and 40 to control the thickness of the tail. The levels for $Z_{n}^{3}$ are slightly larger for thicker tails, while $T_{n}^{3}$ is quite robust to the thick tail of the underlying distribution.

\subsection{Unbalanced, Randomized Two-Sample Designs}

For a balanced, randomized two-sample design, the sample sizes for the control and treatment are assumed to be equal to $n$. Hence, we can randomly form $n$ pairs of sets and rank the units within each pair. In this way, all $n$ pairs are divided into two types of sets. The JPS estimator $\hat{\Delta}_{1}$ is obtained by averaging the means of the differences of responses for the two types of sets. This randomization and ranking procedure heavily depends on the assumption of equal sample sizes for the control and treatment. Since the RBE is defined as the average of $n$ ! possible $\hat{\Delta}_{1}$ 's, it also requires equal sample sizes. The rise of $\operatorname{VRBE} \hat{\Delta}_{3}$, however, makes the extension 
from balanced, randomized two-sample design to unbalanced two-sample design more natural.

\subsection{1 $\hat{\Delta}_{3}$ for Unbalanced, Randomized Two-Sample Designs}

Define $n_{1}$ and $n_{2}$ to be the sample sizes for the control and treatment respectively. For an unbalanced two-sample design, we have different sample sizes, i.e. $n_{1} \neq n_{2}$. Without loss of generosity, we can assume $n_{1}<n_{2}$. In this case, the number of possible pairs that need to be ranked is $n_{1} \times n_{2}$. As before, denote the ranking result for the pair of $Y_{1 i}-Y_{2 j}$ by $R_{i j}$, and let $d_{i j}=Y_{1 i}-Y_{2 j}$, where $i=1,2, \cdots, n_{1}, j=$ $1,2, \cdots, n_{2}$. Let $S_{n_{1} n_{2}}$ denote the collection of Type 1 sets and $l_{1}$ denote the number of Type 1 sets among all the possible $n_{1} n_{2}$ pairs, so we have

$$
R_{i j}= \begin{cases}1 & \text { when }\left(Y_{1 i}, Y_{2 j}\right) \in S_{n_{1} n_{2}} \\ 0 & \text { when }\left(Y_{1 i}, Y_{2 j}\right) \notin S_{n_{1} n_{2}}\end{cases}
$$

and $l_{1}=\sum_{i=1}^{n_{1}} \sum_{j=1}^{n_{2}} R_{i j}, i=1,2, \cdots, n_{1}, j=1,2, \cdots, n_{2}$. According to the definition of the four-dimensional random vector $\mathbf{W}_{i j}$, the VRBE $\hat{\Delta}_{3}$ for an unbalanced, randomized two-sample design can be derived by applying the function $G(x)=\frac{1}{2}\left(\frac{x_{1}}{x_{3}}+\frac{x_{2}}{x_{4}}\right)$ on the average of $\mathbf{W}_{i j}$ 's. That is $\hat{\Delta}_{3}=G\left(\mathbf{W}_{n_{1} n_{2}}\right)$, where

$$
\mathbf{W}_{n_{1} n_{2}}=\left[\frac{l_{1}}{n_{1} n_{2}} \frac{1}{l_{1}} \sum_{d_{i j} \in S_{n_{1} n_{2}}} d_{i j}, \frac{n_{1} n_{2}-l_{1}}{n_{1} n_{2}} \frac{1}{n_{1} n_{2}-l_{1}} \sum_{d_{i j} \notin S_{n_{1} n_{2}}} d_{i j}, \frac{l_{1}}{n_{1} n_{2}}, \frac{n_{1} n_{2}-l_{1}}{n_{1} n_{2}}\right] .
$$

The corresponding expectation of $\mathbf{W}_{n_{1} n_{2}}$ is

$$
E\left(\mathbf{W}_{n_{1} n_{2}} \mid l_{1}\right)=\left[\frac{l_{1}}{n_{1} n_{2}} \delta_{1}, \frac{n_{1} n_{2}-l_{1}}{n_{1} n_{2}} \delta_{2}, \frac{l_{1}}{n_{1} n_{2}}, \frac{n_{1} n_{2}-l_{1}}{n_{1} n_{2}}\right]^{T} .
$$

Applying the Taylor series expansion and replacing $x$ and $x_{0}$ by $\mathbf{W}_{n_{1} n_{2}}$ and $E\left(\mathbf{W}_{n_{1} n_{2}} \mid l_{1}\right)$, respectively, lead us to conclude that $\hat{\Delta}_{3}$ is an asymptotically unbiased estimator of $\mu_{1}-\mu_{2}$ for an unbalanced, randomized two-sample design with the variance matrix $G^{\prime} \Sigma_{n_{1} n_{2}} G^{\prime T}$, where $\operatorname{Var}\left(\mathbf{W}_{n_{1} n_{2}}\right)=\Sigma_{n_{1} n_{2}}$. 
As in balanced, two-sample problems, the VRBE $\hat{\Delta}_{3}$ in an unbalanced, two-sample problem still has limiting normal distribution as the sample sizes for the control and treatment become increasingly large. The proof follows the same idea as in the balance case, i.e. applying the asymptotic properties of generalized U-statistics. As before, let $C_{1}, C_{2}, \cdots, C_{n_{1}}$ be two dimensional random vectors that are i.i.d. from the control population, i.e., $C_{i}=\left(Y_{1 i}, X_{1 i}\right)^{T} ; i=1,2, \cdots, n_{1}$. Similarly, define $T_{1}, T_{2}, \cdots, T_{n_{2}}$ to be the random vectors that are i.i.d. from the treatment population where $T_{j}=\left(Y_{2 j}, X_{2 j}\right)^{T} ; j=1,2, \cdots, n_{2}$. Here $\mathbf{W}_{n_{1} n_{2}}$ can be written as $U_{n_{1} n_{2}}=$ $\left[\frac{1}{n_{1} n_{2}} \sum_{i=1}^{n_{1}} \sum_{j=1}^{n_{2}}\left(Y_{1 i}-Y_{2 j}\right) I\left(X_{1 i}<X_{2 j}\right), \frac{1}{n_{1} n_{2}} \sum_{i=1}^{n_{1}} \sum_{j=1}^{n_{2}}\left(Y_{1 i}-Y_{2 j}\right) I\left(X_{1 i}>X_{2 j}\right)\right.$, $\left.\frac{1}{n_{1} n_{2}} \sum_{i=1}^{n_{1}} \sum_{j=1}^{n_{1}} I\left(X_{1 i}<X_{2 j}\right), \frac{1}{n_{1} n_{2}} \sum_{i=1}^{n_{1}} \sum_{j=1}^{n_{2}} I\left(X_{1 i}>X_{2 j}\right)\right]^{T}$. Then $\mathbf{W}_{n_{1} n_{2}}$ is also a four dimensional, two-sample generalized U-statistic with

$$
U_{n_{1} n_{2}}^{(1)}=\frac{1}{n_{1} n_{2}} \sum_{i=1}^{n_{1}} \sum_{j=1}^{n_{2}}\left(Y_{1 i}-Y_{2 j}\right) I\left(X_{1 i}<X_{2 j}\right)=\frac{1}{\left(\begin{array}{c}
n_{1} \\
1
\end{array}\right)\left(\begin{array}{c}
n_{2} \\
1
\end{array}\right)} \sum_{i=1}^{n_{1}} \sum_{j=1}^{n_{2}} \psi^{(1)}\left(C_{i}, T_{j}\right),
$$

where

$$
\psi^{(1)}\left(C_{i}, T_{j}\right)=\left(Y_{1 i}-Y_{2 j}\right) I\left(X_{1 i}<X_{2 j}\right),
$$

is the kernel for $U^{(1)}$ and is symmetric in $C_{1}, \cdots, C_{n_{1}}$ and $T_{1}, \cdots, T_{n_{2}}$, with $\theta_{n_{1} n_{2}}^{(1)}=$ $E\left(\psi^{(1)}\left(C_{i}, T_{j}\right)\right)=\frac{1}{2} \delta_{1}$. Similarly,

$$
\begin{aligned}
& U_{n_{1} n_{2}}^{(2)}=\frac{1}{n_{1} n_{2}} \sum_{i=1}^{n_{1}} \sum_{j=1}^{n_{2}}\left(Y_{1 i}-Y_{2 j}\right) I\left(X_{1 i}>X_{2 j}\right)=\frac{1}{\left(\begin{array}{c}
n_{1} \\
1
\end{array}\right)\left(\begin{array}{c}
n_{2} \\
1
\end{array}\right)} \sum_{i=1}^{n_{1}} \sum_{j=1}^{n_{2}} \psi^{(2)}\left(C_{i}, T_{j}\right), \\
& U_{n_{1} n_{2}}^{(3)}=\frac{1}{n_{1} n_{2}} \sum_{i=1}^{n_{1}} \sum_{j=1}^{n_{2}} I\left(X_{1 i}<X_{2 j}\right)=\frac{1}{\left(\begin{array}{c}
n_{1} \\
1
\end{array}\right)\left(\begin{array}{c}
n_{2} \\
1
\end{array}\right)} \sum_{i=1}^{n_{1}} \sum_{j=1}^{n_{2}} \psi^{(3)}\left(C_{i}, T_{j}\right), \\
& U_{n_{1} n_{2}}^{(4)}=\frac{1}{n_{1} n_{2}} \sum_{i=1}^{n_{1}} \sum_{j=1}^{n_{2}} I\left(X_{1 i}>X_{2 j}\right)=\frac{1}{\left(\begin{array}{c}
n_{1} \\
1
\end{array}\right)\left(\begin{array}{c}
n_{2} \\
1
\end{array}\right)} \sum_{i=1}^{n_{1}} \sum_{j=1}^{n_{2}} \psi^{(4)}\left(C_{i}, T_{j}\right),
\end{aligned}
$$


where

$$
\begin{aligned}
\psi^{(2)}\left(C_{i}, T_{j}\right) & =\left(Y_{1 i}-Y_{2 j}\right) I\left(X_{1 i}>X_{2 j}\right), \\
\psi^{(3)}\left(C_{i}, T_{j}\right) & =I\left(X_{1 i}<X_{2 j}\right), \\
\psi^{(4)}\left(C_{i}, T_{j}\right) & =I\left(X_{1 i}>X_{2 j}\right) .
\end{aligned}
$$

These kernels are symmetric in $C_{1}, \cdots, C_{n_{1}}$ and $T_{1}, \cdots, T_{n_{2}}$, with

$$
\begin{aligned}
& \theta_{n_{1} n_{2}}^{(2)}=E\left(\psi^{(2)}\right)=\frac{1}{2} \delta_{2}, \\
& \theta_{n_{1} n_{2}}^{(3)}=E\left(\psi^{(3)}\right)=\frac{1}{2}, \\
& \theta_{n_{1} n_{2}}^{(4)}=E\left(\psi^{(4)}\right)=\frac{1}{2} .
\end{aligned}
$$

We also define $\theta_{n_{1} n_{2}}^{*}$ to be the expectation of $U_{n_{1} n_{2}}$ conditional on $l_{1}$, where $l_{1}$ indicates the number of Type 1 sets among the $n_{1} n_{2}$ pairs. Then

$$
\theta_{n_{1} n_{2}}^{*}=\left(\theta_{n_{1} n_{2}}^{*(1)}, \theta_{n_{1} n_{2}}^{*(2)}, \theta_{n_{1} n_{2}}^{*(3)}, \theta_{n_{1} n_{2}}^{*(4)}\right),
$$

where

$$
\begin{aligned}
& \theta_{n_{1} n_{2}}^{*(1)}=E\left(\psi^{(1)}\left(C_{i}, T_{j}\right) \mid l_{1}\right)=\frac{l_{1}}{n_{1} n_{2}} \delta_{1}, \\
& \theta_{n_{1} n_{2}}^{*(2)}=E\left(\psi^{(2)} \mid l_{1}\right)=\frac{n_{1} n_{2}-l_{1}}{n_{1} n_{2}} \delta_{2}, \\
& \theta_{n_{1} n_{2}}^{*(3)}=E\left(\psi^{(3)} \mid l_{1}\right)=\frac{l_{1}}{n_{1} n_{2}}, \\
& \theta_{n_{1} n_{2}}^{*(4)}=E\left(\psi^{(4)} \mid l_{1}\right)=\frac{n_{1} n_{2}-l_{1}}{n_{1} n_{2}} .
\end{aligned}
$$

Theorem 3 can be modified to derive the Asymptotic Normality (AN) of $\hat{\Delta}_{3}$ in unbalanced, two-sample problems.

\section{Theorem 4 (AN of $\hat{\Delta}_{3}$ in unbalanced two-sample problems)}

Let $h_{i\left(n_{1} n_{2}\right)}^{(j)} ; i=1,2$, be the set of first kernel functions in the $H$ decomposition of 
$U_{n_{1} n_{2}}^{(j)} ; j=1,2,3,4$, i.e. $h_{i\left(n_{1} n_{2}\right)}^{(j)}(C)=E \psi^{(j)}\left(C, T_{j}\right)-\theta_{n_{1} n_{2}}^{(j)}$. Define $\Sigma_{1}$ and $\Sigma_{2}$ to be the variance matrices of the random vectors

$$
Z_{1\left(n_{1} n_{2}\right), i}=\left(h_{1\left(n_{1} n_{2}\right)}^{(1)}\left(C_{i}\right), h_{1\left(n_{1} n_{2}\right)}^{(2)}\left(C_{i}\right), h_{1\left(n_{1} n_{2}\right)}^{(3)}\left(C_{i}\right), h_{1\left(n_{1} n_{2}\right)}^{(4)}\left(C_{i}\right)\right), i=1,2, \cdots, n_{1},
$$

and

$$
Z_{2\left(n_{1} n_{2}\right), j}=\left(h_{2\left(n_{1} n_{2}\right)}^{(1)}\left(T_{j}\right), h_{2\left(n_{1} n_{2}\right)}^{(2)}\left(T_{j}\right), h_{2\left(n_{1} n_{2}\right)}^{(3)}\left(T_{j}\right), h_{2\left(n_{1} n_{2}\right)}^{(4)}\left(T_{j}\right)\right) j=1,2, \cdots, n_{2}
$$

respectively. Assume all the variances are finite. Then as $\min \left(n_{1}, n_{2}\right) \rightarrow \infty$, we have

$$
\frac{\sqrt{n_{1}+n_{2}}\left(\hat{\Delta}_{3}-\Delta\right)}{G^{\prime}\left(\theta_{n_{1} n_{2}}^{*}\right)\left(\frac{n_{1}+n_{2}}{n_{1}} \Sigma_{1}+\frac{n_{1}+n_{2}}{n_{2}} \Sigma_{2}\right) G^{\prime T}\left(\theta_{n_{1} n_{2}}^{*}\right)} \stackrel{D}{\rightarrow} N(0,1),
$$

where $G^{\prime}\left(\theta_{n_{1} n_{2}}^{*}\right)$ is the first derivative of $G(x)=\frac{1}{2}\left(\frac{x_{1}}{x_{3}}+\frac{x_{2}}{x_{4}}\right)$ evaluated at

$$
\theta_{n_{1} n_{2}}^{*}=\left(\theta_{\left(n_{1} n_{2}\right)}^{*(1)}, \theta_{\left(n_{1} n_{2}\right)}^{*(2)}, \theta_{\left(n_{1} n_{2}\right)}^{*(3)}, \theta_{\left(n_{1} n_{2}\right)}^{*(4)}\right)
$$

Proof of Theorem 4: Suppose $\min \left(n_{1}, n_{2}\right) \rightarrow \infty$ in such a way that $n_{i} / N \rightarrow$ $p_{i}>0$, where $N=n_{1}+n_{2}$. Also let $\theta_{n_{1} n_{2}}=\left(\theta_{n_{1} n_{2}}^{(1)}, \theta_{n_{1} n_{2}}^{(2)}, \theta_{n_{1} n_{2}}^{(3)}, \theta_{n_{1} n_{2}}^{(4)}\right)$ and $\theta_{n_{1} n_{2}}^{*}=$ $\left(\theta_{n_{1} n_{2}}^{*(1)}, \theta_{n_{1} n_{2}}^{*(2)}, \theta_{n_{1} n_{2}}^{*(3)}, \theta_{n_{1} n_{2}}^{*(4)}\right)$. Then by theorem of multivariate normal distribution for generalized U-statistics (Lee 1990), we have $\sqrt{N}\left(U_{n_{1} n_{2}}-\theta_{n_{1} n_{2}}\right)$ converges in distribution to multivariate normal distribution with mean vector zero and covariance matrix $\Sigma_{n_{1} n_{2}}=p_{1}^{-1} k_{1}^{2} \Sigma_{1}+p_{2}^{-1} k_{2}^{2} \Sigma_{2}=\left(\frac{n_{1}+n_{2}}{n_{1}} \Sigma_{1}+\frac{n_{1}+n_{2}}{n_{2}} \Sigma_{2}\right)$.

Let $G(x)=\frac{1}{2}\left(\frac{x_{1}}{x_{3}}+\frac{x_{2}}{x_{4}}\right)$, where $x=\left[x_{1}, x_{2}, x_{3}, x_{4}\right]^{T}$. If we apply $G(x)$ on asymptotic distribution of the random vector $\left(U_{n_{1} n_{2}}-\theta_{n_{1} n_{2}}\right)$, the following result is ready to obtain by the Delta method.

$$
\sqrt{N}\left(G\left(U_{n_{1} n_{2}}\right)-G\left(\theta_{n_{1} n_{2}}\right)\right)=\sqrt{N}\left(\hat{\Delta}_{3}-\Delta\right) \underset{D}{D} N\left(0, G^{\prime}\left(\theta_{n_{1} n_{2}}\right) \Sigma_{n_{1} n_{2}} G^{\prime T}\left(\theta_{n_{1} n_{2}}\right),\right.
$$

where $G^{\prime}\left(\theta_{n_{1} n_{2}}\right)$ is the first derivative of $G(x)$ evaluated at $\theta_{n_{1} n_{2}}$, i.e.

$$
G^{\prime}\left(\theta_{n_{1} n_{2}}\right)=\left(1,1,-\delta_{1},-\delta_{2}\right)
$$


Then we have

$$
\frac{\sqrt{N}\left(\hat{\Delta}_{3}-\Delta\right)}{G^{\prime}\left(\theta_{n_{1} n_{2}}\right) \Sigma_{n_{1} n_{2}} G^{\prime T}\left(\theta_{n_{1} n_{2}}\right)} \stackrel{D}{\rightarrow} N(0,1) .
$$

Apply the Slutsky's Theorem, we have

$$
\frac{\sqrt{N}\left(\hat{\Delta}_{3}-\Delta\right)}{G^{\prime}\left(\theta_{n_{1} n_{2}}^{*}\right) \Sigma_{n_{1} n_{2}} G^{\prime T}\left(\theta_{n_{1} n_{2}}^{*}\right)} \stackrel{D}{\rightarrow} N(0,1)
$$

where

$$
G^{\prime}\left(\theta_{n_{1} n_{2}}^{*}\right)=\left(\frac{n_{1} n_{2}}{2 l_{1}}, \frac{n_{1} n_{2}}{2\left(n_{1} n_{2}-l_{1}\right)},-\frac{n_{1} n_{2}}{2 l_{1}} \delta_{1},-\frac{n_{1} n_{2}}{2\left(n_{1} n_{2}-l_{1}\right)} \delta_{2}\right) .
$$

\subsection{2 $\hat{\Delta}_{1}$ for Unbalanced, Randomized Two-Sample Designs}

The randomization and ranking procedure for $\hat{\Delta}_{1}$ requires $n$ sets randomly formed and ranked. Without loss of generosity, assume that the control has smaller sample size than the treatment, i.e. $n_{1}<n_{2}$. To obtain $\hat{\Delta}_{1}$, we randomly choose $n_{1}$ observations out of $n_{2}$ from the treatment. Then we have equal sample size $n=n_{1}$ for the control and treatment. In this way, the JPS $\hat{\Delta}_{1}$ and the RBE $\hat{\Delta}_{2}$ can be obtain for unbalanced, randomized two-sample problems. However, we are losing information by throwing away some observations for the treatment.

Another possibility is to first calculate $\left(\begin{array}{l}n_{2} \\ n_{1}\end{array}\right) \hat{\Delta}_{1}$ 's corresponding to $\left(\begin{array}{l}n_{2} \\ n_{1}\end{array}\right)$ selections of $n_{1}$ observations from the treatment. Then we can average these $\hat{\Delta}_{1}$ 's to form a new estimator. However, since this new estimator involves very complicated correlation structure, we do not pursue it further. 

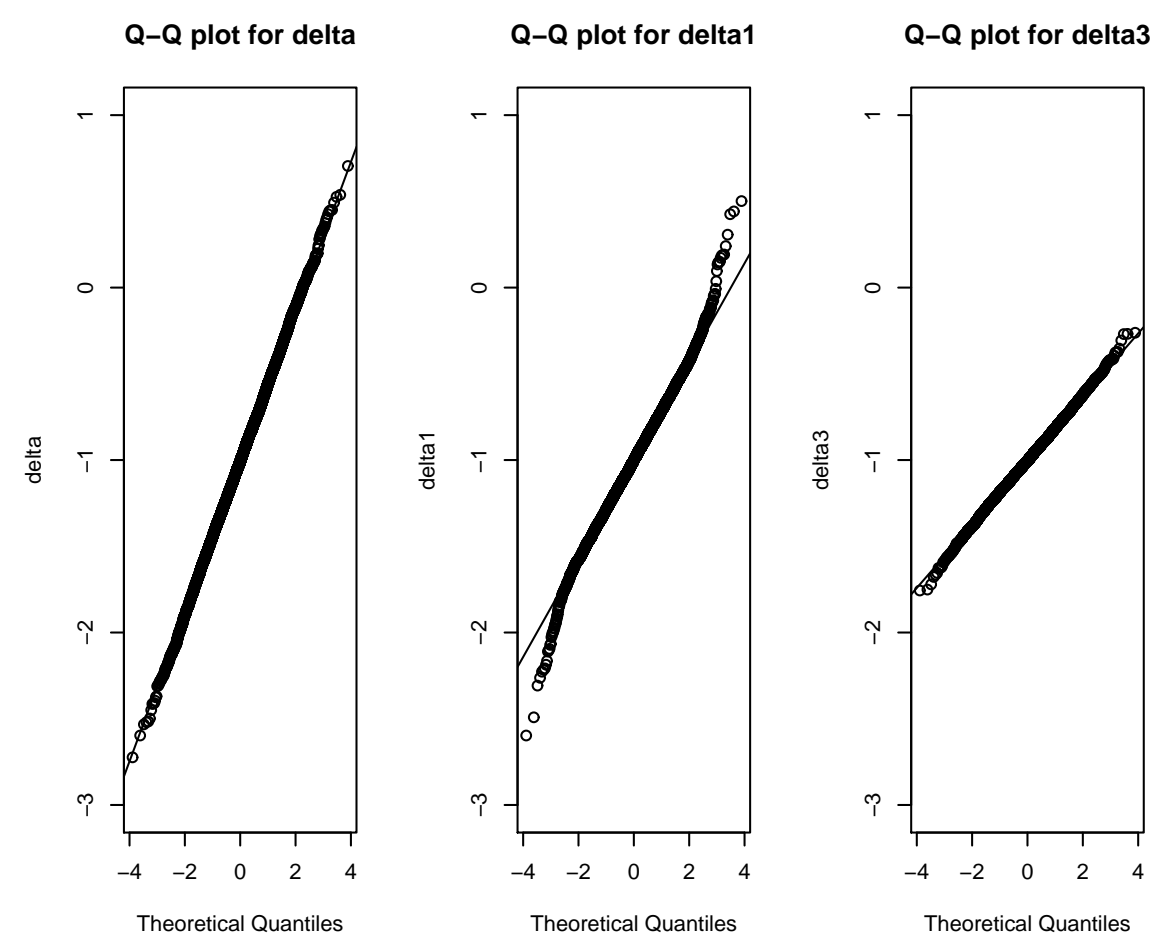

Figure 3.1: Normal Probability plots of $\hat{\Delta}, \hat{\Delta}_{1}$, and $\hat{\Delta}_{3}$ for perfect ranking when $n=10$ 


\begin{tabular}{ccccccccc}
\hline$\rho$ & $n$ & $T_{n}\left(\hat{\Delta}_{1}\right)$ & $Z_{n}\left(\hat{\Delta}_{1}\right)$ & $S_{n}\left(\hat{\Delta}_{1}\right)$ & $T_{n}^{S R S}(\hat{\Delta})$ & $Z_{n}^{S R S}(\hat{\Delta})$ & $Z_{n}^{3}\left(\hat{\Delta}_{3}\right)$ & $T_{n}^{3}\left(\hat{\Delta}_{3}\right)$ \\
\hline 1 & 10 & 0.0464 & 0.1097 & 0.0613 & 0.0502 & 0.0640 & 0.0737 & 0.0398 \\
& 30 & 0.0497 & 0.0616 & 0.0494 & 0.0479 & 0.0521 & 0.0549 & 0.0458 \\
& 50 & 0.0478 & 0.0534 & 0.0469 & 0.0454 & 0.0490 & 0.0492 & 0.0438 \\
& 100 & 0.0483 & 0.0516 & 0.0486 & 0.0489 & 0.0511 & 0.0502 & 0.0472 \\
\hline 0.7 & 10 & 0.0463 & 0.1108 & 0.0586 & 0.0502 & 0.0640 & 0.0720 & 0.0437 \\
& 30 & 0.0490 & 0.0606 & 0.0477 & 0.0479 & 0.0521 & 0.0603 & 0.0490 \\
& 50 & 0.0469 & 0.0538 & 0.0461 & 0.0454 & 0.0490 & 0.0504 & 0.0449 \\
& 100 & 0.0518 & 0.0560 & 0.0528 & 0.0489 & 0.0511 & 0.0519 & 0.0486 \\
\hline 0.5 & 10 & 0.0470 & 0.1085 & 0.0597 & 0.0502 & 0.0640 & 0.0758 & 0.0431 \\
& 30 & 0.0528 & 0.0625 & 0.0502 & 0.0479 & 0.0521 & 0.0588 & 0.0483 \\
& 50 & 0.0457 & 0.0507 & 0.0447 & 0.0454 & 0.0490 & 0.0498 & 0.0454 \\
& 100 & 0.0487 & 0.0516 & 0.0484 & 0.0489 & 0.0511 & 0.0511 & 0.0481 \\
\hline 0 & 10 & 0.0477 & 0.1062 & 0.0577 & 0.0502 & 0.0640 & 0.0761 & 0.0445 \\
& 30 & 0.0483 & 0.0632 & 0.0501 & 0.0479 & 0.0521 & 0.0550 & 0.0468 \\
& 50 & 0.0468 & 0.0531 & 0.0470 & 0.0454 & 0.0490 & 0.0496 & 0.0437 \\
& 100 & 0.0494 & 0.0519 & 0.0492 & 0.0489 & 0.0511 & 0.0497 & 0.0474 \\
\hline
\end{tabular}

Table 3.3: Estimated levels of a nominal $\alpha=0.05$ test for Student's $t$-distribution $\left(T_{n}\right)$ with $n-2$ d.f., the Limiting normal distribution $\left(Z_{n}\right)$, and Satterthwaite's approximation $\left(S_{n}\right)$ for $\hat{\Delta}_{1}$, Student's $t$-distribution $\left(T_{n}^{S R S}\right)$ with $2 n-2$ d.f. and the normal distribution $\left(Z_{n}^{S R S}\right)$ for $\hat{\Delta}$, the Limiting normal distribution $\left(Z_{n}^{3}\right)$ and Student's $t$-distribution $\left(T_{n}^{3}\right)$ with $n-1$ d.f. for $\hat{\Delta}_{3}$. Simulations are based on 10,000 replicates, scale parameters are set to 1 . Standard errors range from 0.001 to 0.003 . 

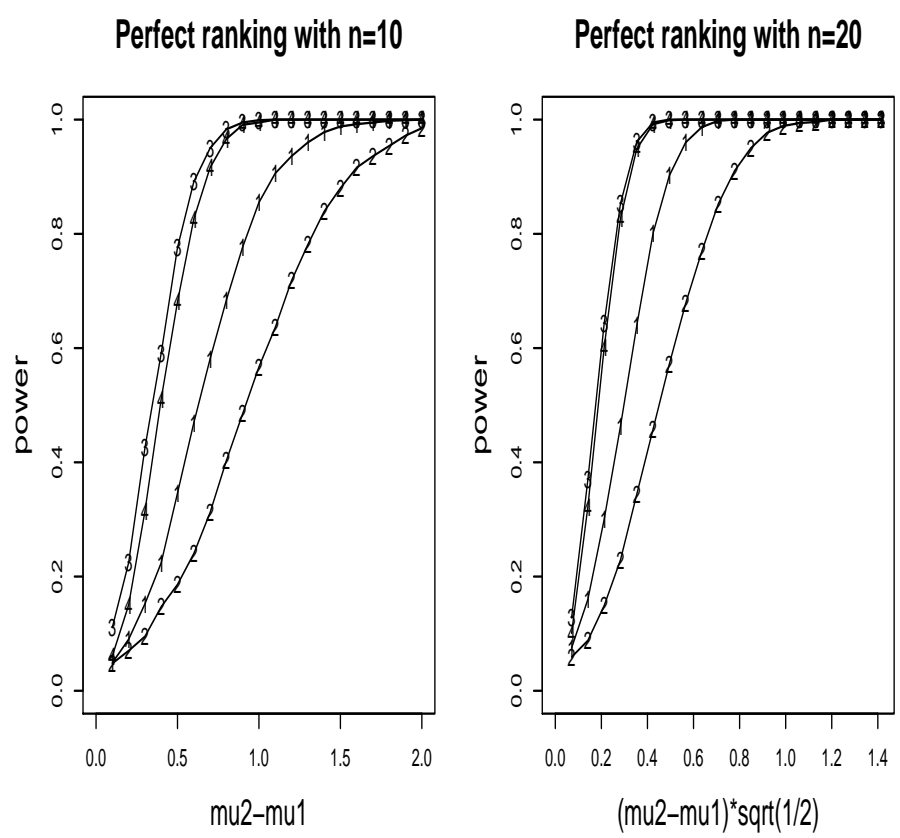

Perfect ranking with $n=30$

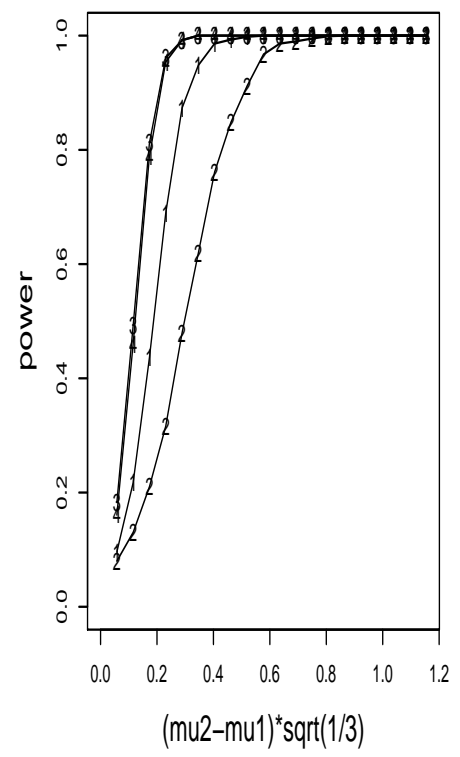

Perfect ranking with $n=40$

Perfect ranking with $\mathrm{n}=50$
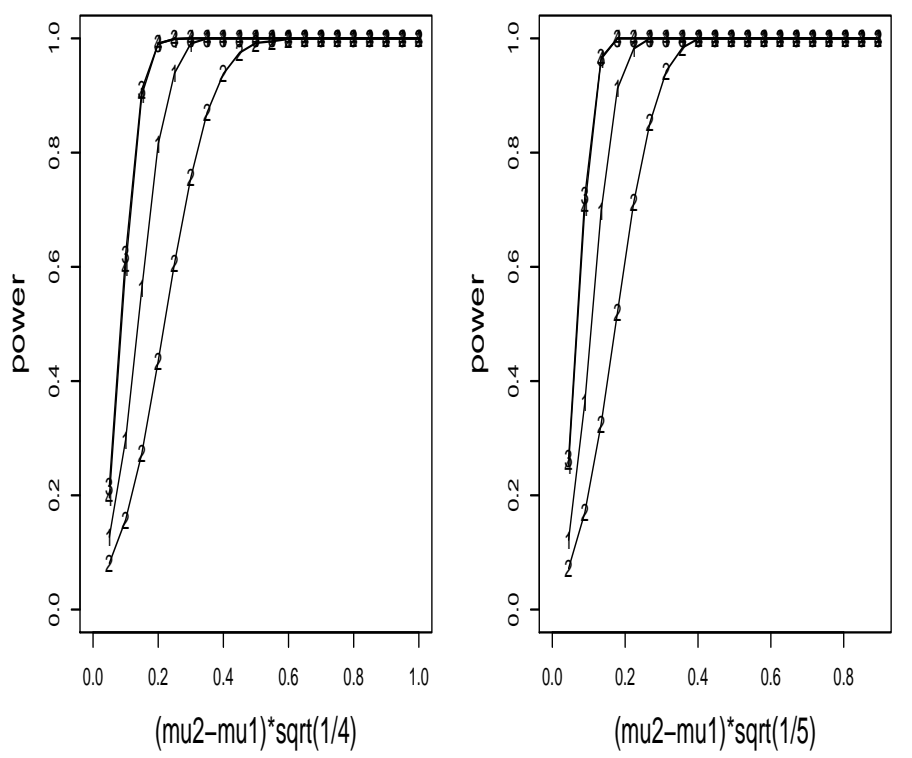

Figure 3.2: Empirical power of $T_{n}$ for $\hat{\Delta}_{1}$ with symbol $1, T_{n}^{S R S}$ for $\hat{\Delta}$ with symbol 2, $Z_{n}^{3}$ and $T_{n}^{3}$ for $\hat{\Delta}_{3}$ with symbols 3 and 4 , respectively, under perfect ranking. 

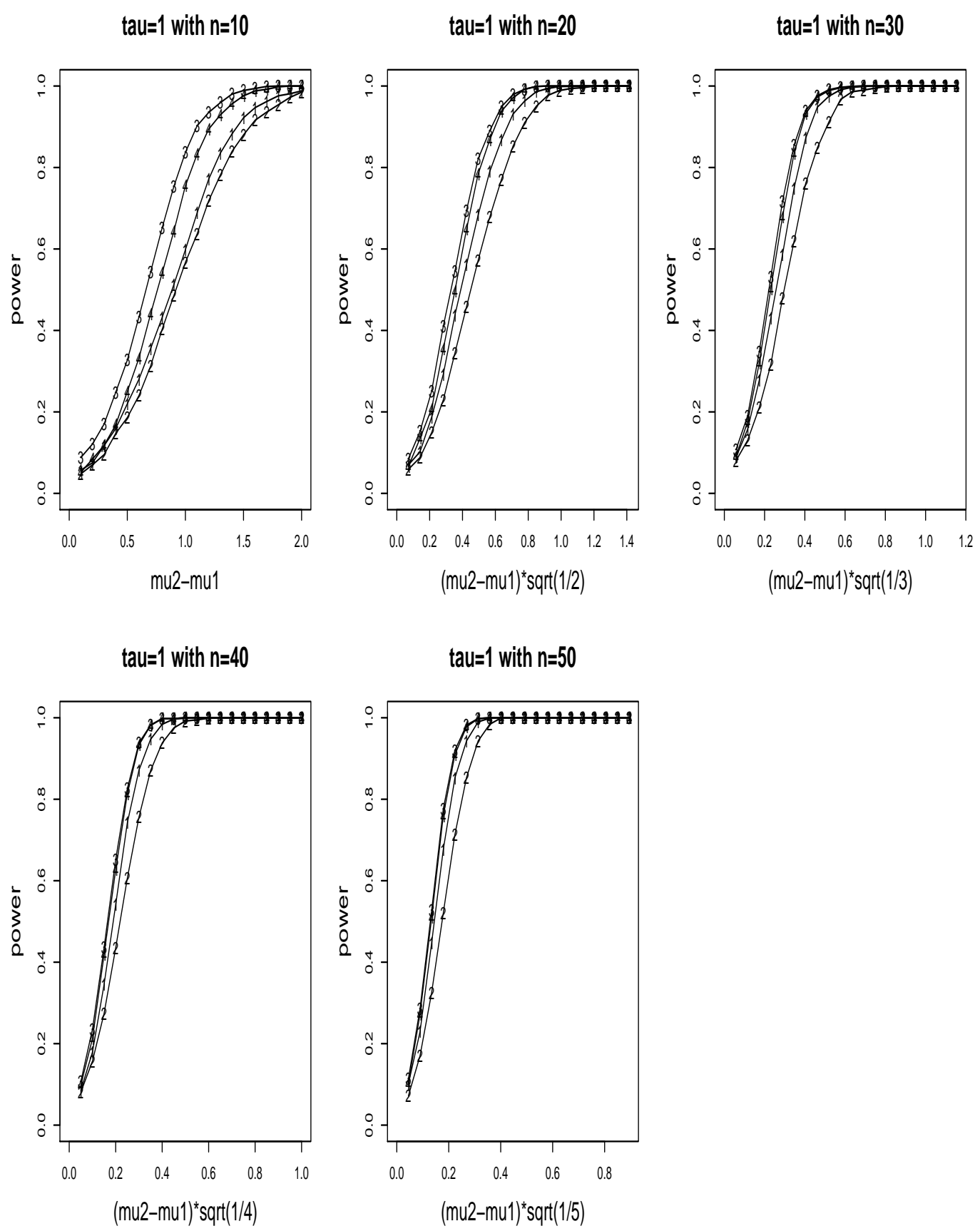

Figure 3.3: Empirical power of $T_{n}$ for $\hat{\Delta}_{1}$ with symbol $1, T_{n}^{S R S}$ for $\hat{\Delta}$ with symbol 2, $Z_{n}^{3}$ and $T_{n}^{3}$ for $\hat{\Delta}_{3}$ with symbols 3 and 4 , respectively, when $\tau^{2}=1$. 

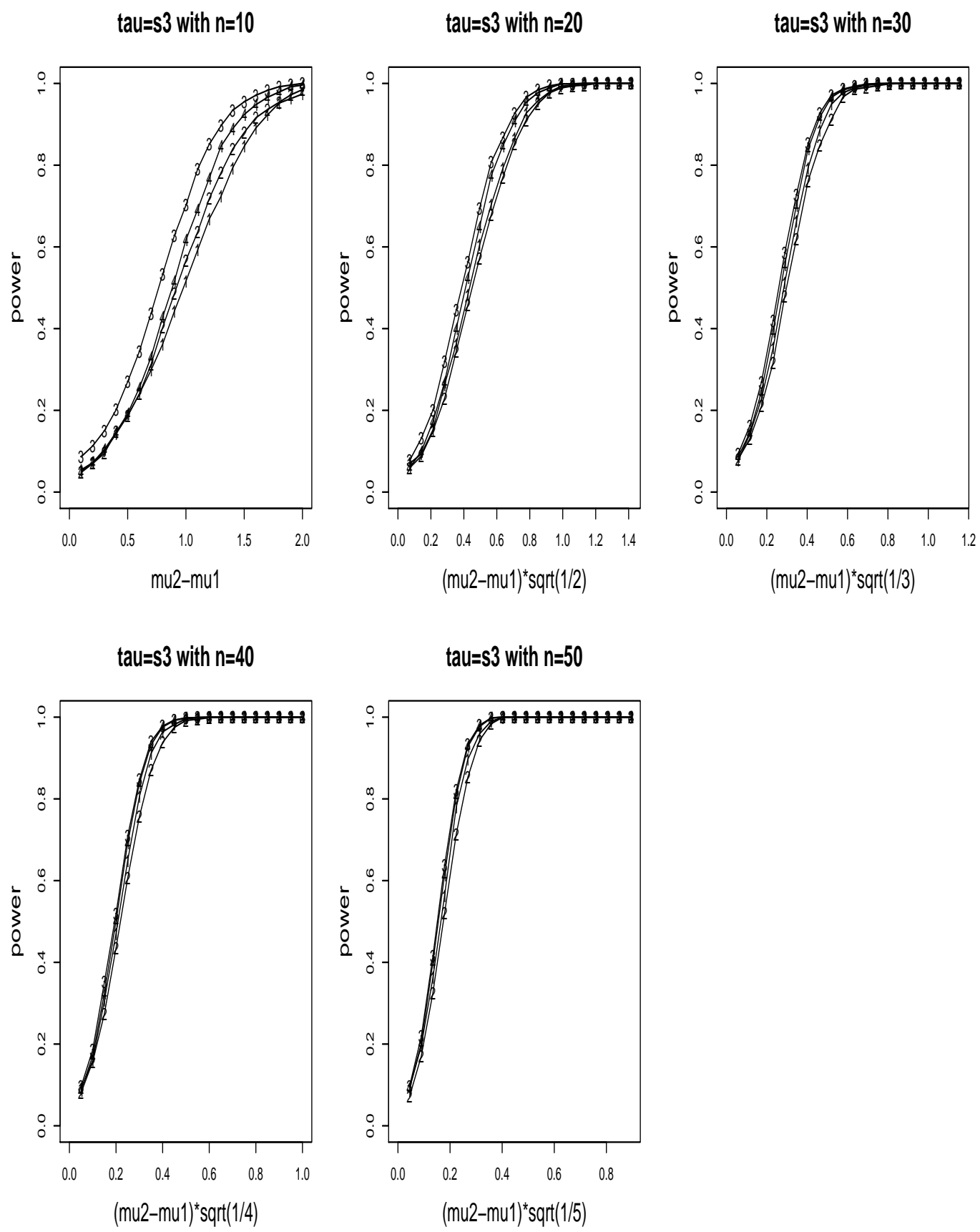

Figure 3.4: Empirical power of $T_{n}$ for $\hat{\Delta}_{1}$ with symbol $1, T_{n}^{S R S}$ for $\hat{\Delta}$ with symbol 2, $Z_{n}^{3}$ and $T_{n}^{3}$ for $\hat{\Delta}_{3}$ with symbols 3 and 4 , respectively, when $\tau^{2}=3$. 

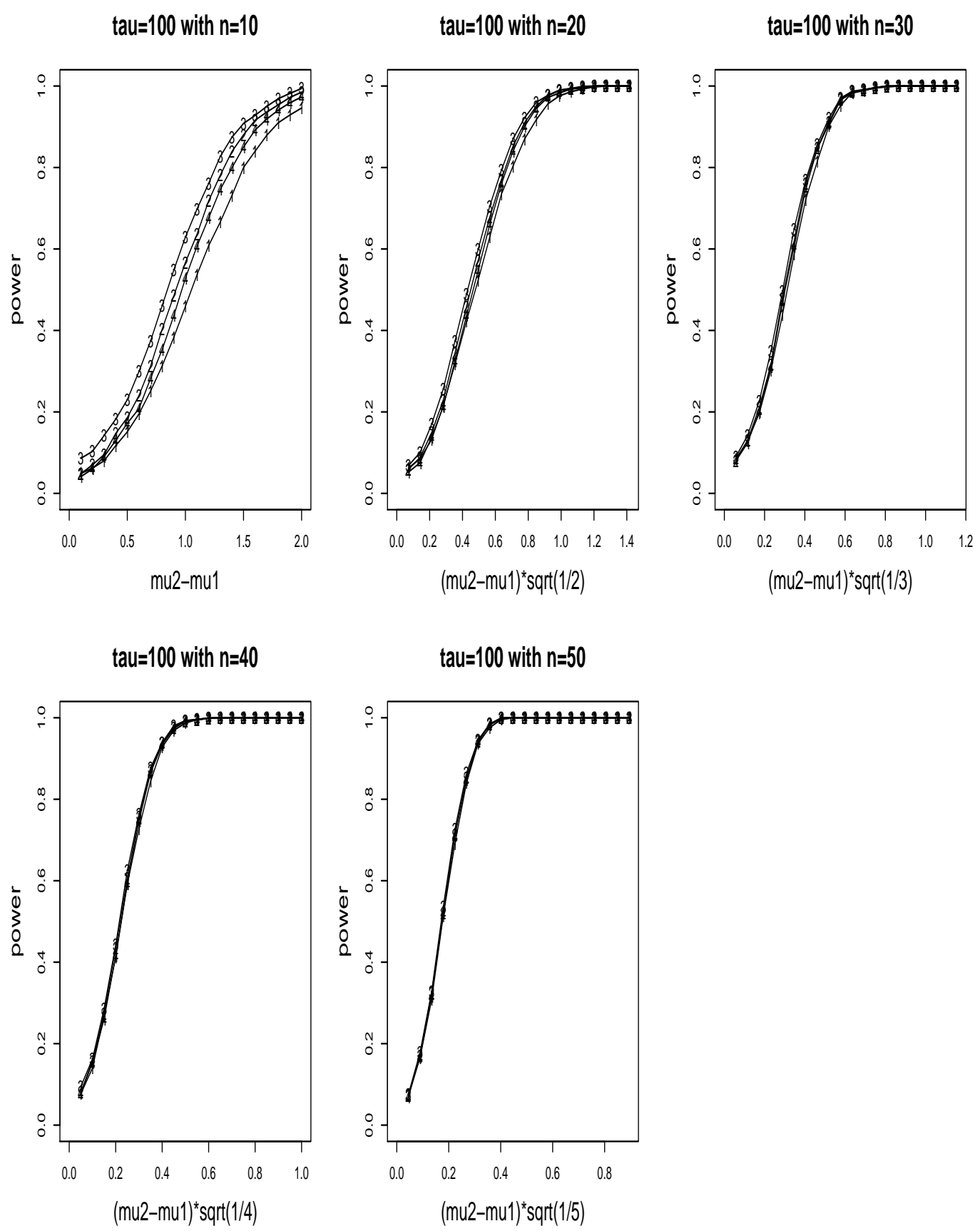

Figure 3.5: Empirical power of $T_{n}$ for $\hat{\Delta}_{1}$ with symbol $1, T_{n}^{S R S}$ for $\hat{\Delta}$ with symbol 2, $Z_{n}^{3}$ and $T_{n}^{3}$ for $\hat{\Delta}_{3}$ with symbols 3 and 4 , respectively, when $\tau^{2}=10000$. 


\begin{tabular}{ccccccccc}
\hline$\rho$ & $n$ & $T_{n}\left(\hat{\Delta}_{1}\right)$ & $Z_{n}\left(\hat{\Delta}_{1}\right)$ & $S_{n}\left(\hat{\Delta}_{1}\right)$ & $T_{n}^{S R S}(\hat{\Delta})$ & $Z_{n}^{S R S}(\hat{\Delta})$ & $Z_{n}^{3}\left(\hat{\Delta}_{3}\right)$ & $T_{n}^{3}\left(\hat{\Delta}_{3}\right)$ \\
\hline 1 & 10 & 0.0491 & 0.1090 & 0.0579 & 0.0577 & 0.0749 & 0.0876 & 0.0543 \\
& 30 & 0.0515 & 0.0642 & 0.0514 & 0.0558 & 0.0617 & 0.0625 & 0.0520 \\
& 50 & 0.0502 & 0.0565 & 0.0502 & 0.0480 & 0.0502 & 0.0543 & 0.0486 \\
& 100 & 0.0471 & 0.0489 & 0.0471 & 0.0494 & 0.0512 & 0.0520 & 0.0491 \\
\hline 0.7 & 10 & 0.0515 & 0.1138 & 0.0633 & 0.0577 & 0.0749 & 0.0862 & 0.0531 \\
& 30 & 0.0497 & 0.0617 & 0.0492 & 0.0558 & 0.0617 & 0.0622 & 0.0525 \\
& 50 & 0.0488 & 0.0555 & 0.0487 & 0.0480 & 0.0502 & 0.0518 & 0.0477 \\
& 100 & 0.0499 & 0.0533 & 0.0501 & 0.0494 & 0.0512 & 0.0508 & 0.0489 \\
\hline 0.5 & 10 & 0.0491 & 0.1125 & 0.0610 & 0.0577 & 0.0749 & 0.0843 & 0.0539 \\
& 30 & 0.0495 & 0.0645 & 0.0504 & 0.0558 & 0.0617 & 0.0639 & 0.0534 \\
& 50 & 0.0462 & 0.0531 & 0.0466 & 0.0480 & 0.0502 & 0.0531 & 0.0485 \\
& 100 & 0.0505 & 0.0538 & 0.0498 & 0.0494 & 0.0512 & 0.0517 & 0.0478 \\
\hline 0 & 10 & 0.0474 & 0.1093 & 0.0597 & 0.0577 & 0.0749 & 0.0859 & 0.0517 \\
& 30 & 0.0506 & 0.0635 & 0.0505 & 0.0558 & 0.0617 & 0.0617 & 0.0515 \\
& 50 & 0.0453 & 0.0514 & 0.0454 & 0.0480 & 0.0502 & 0.0510 & 0.0461 \\
& 100 & 0.0474 & 0.0498 & 0.0476 & 0.0494 & 0.0512 & 0.0523 & 0.0495 \\
\hline
\end{tabular}

Table 3.4: Estimated levels of a nominal $\alpha=0.05$ test for Student's $t$-distribution $\left(T_{n}\right)$ with $n-2$ d.f., the Limiting normal distribution $\left(Z_{n}\right)$, and Satterthwaite's approximation $\left(S_{n}\right)$ of $\hat{\Delta}_{1}$, Student's $t$-distribution $\left(T_{n}^{S R S}\right)$ with $2 n-2$ d.f. and the normal distribution $\left(Z_{n}^{S R S}\right)$ of $\hat{\Delta}$, the Limiting normal distribution $\left(Z_{n}^{3}\right)$ and Student's $t$-distribution $\left(T_{n}^{3}\right) n-1$ d.f. of $\hat{\Delta}_{3}$. Simulations are based on 10,000 replicates, $\sigma_{1}$ and $\sigma_{2}$ are set to 1 and 4, respectively. Standard errors range from 0.002 to 0.003 . 

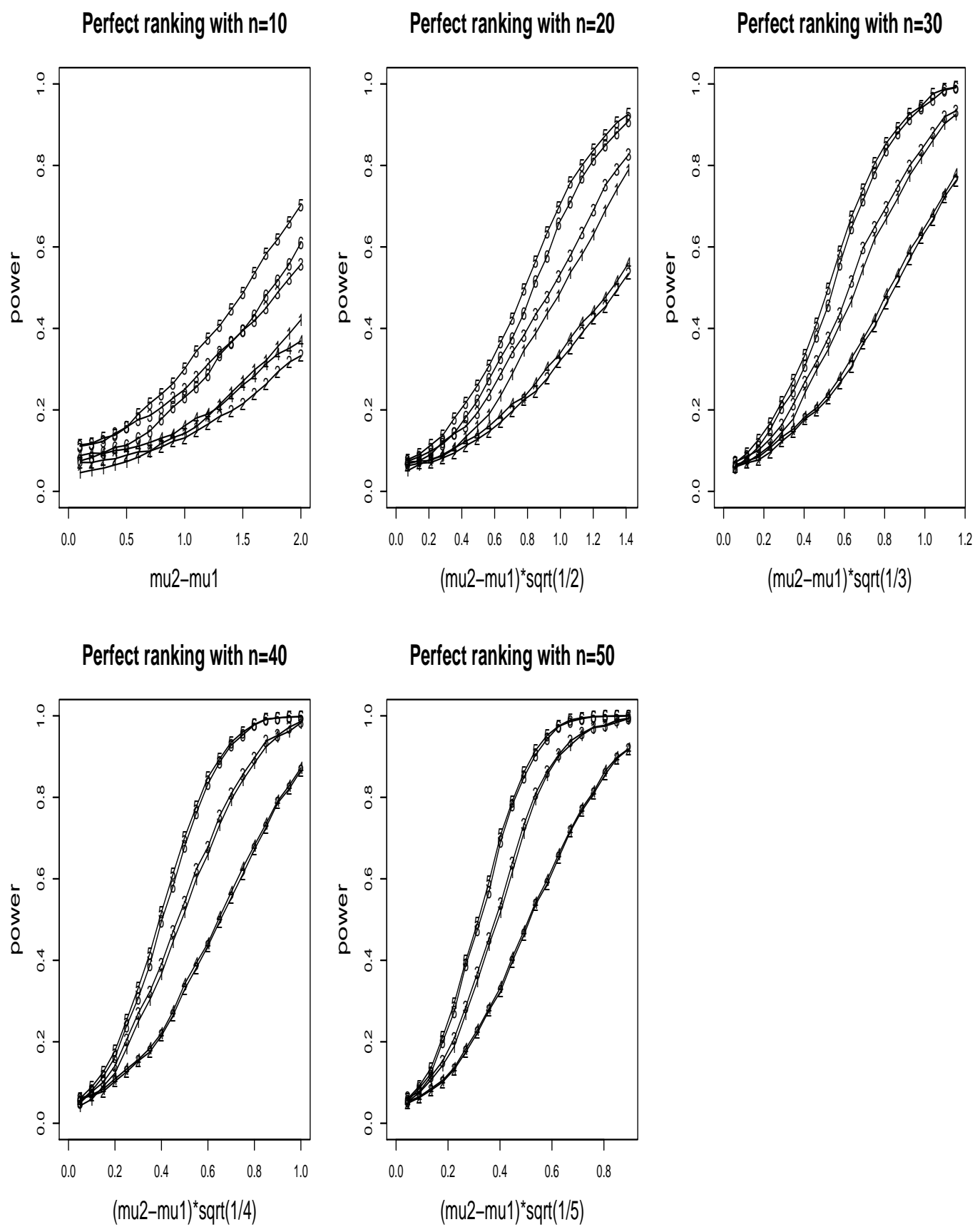

Figure 3.6: Empirical power of test statistics for $\hat{\Delta}_{1}$ including the $t$-distribution with $n-2$ d.f. and the normal approximation (indicated by labels 1 and 3 respectively) and test statistics for $\hat{\Delta}$ including the $t$-distribution with $2 n-2$ d.f. and the normal approximation (indicated by labels 2 and 4 respectively), and test statistics for $\hat{\Delta}_{3}$ including the normal approximation and the $t$-distribution with $n-1$ d.f. (indicated by labels 5 and 6 respectively) under perfect ranking. The simulations are based on 10,000 replicates. $\sigma_{1}$ and $\sigma_{2}$ are set to 1 and 4 , respectively. 


\begin{tabular}{ccccccccc}
\hline$\sigma$ & $n$ & $T_{n}\left(\hat{\Delta}_{1}\right)$ & $Z_{n}\left(\hat{\Delta}_{1}\right)$ & $S_{n}\left(\hat{\Delta}_{1}\right)$ & $T_{n}^{S R S}(\hat{\Delta})$ & $Z_{n}^{S R S}(\hat{\Delta})$ & $Z_{n}^{3}\left(\hat{\Delta}_{3}\right)$ & $T_{n}^{3}\left(\hat{\Delta}_{3}\right)$ \\
\hline 0.5 & 10 & 0.0423 & 0.1027 & 0.0493 & 0.0460 & 0.0590 & 0.0795 & 0.0408 \\
& 30 & 0.0460 & 0.0601 & 0.0463 & 0.0473 & 0.0521 & 0.0650 & 0.0528 \\
& 50 & 0.0440 & 0.0496 & 0.0431 & 0.0467 & 0.0498 & 0.0577 & 0.0508 \\
& 100 & 0.0432 & 0.0457 & 0.0426 & 0.0462 & 0.0475 & 0.0521 & 0.0492 \\
\hline 1 & 10 & 0.0369 & 0.0870 & 0.0382 & 0.0332 & 0.0475 & 0.0780 & 0.0399 \\
& 30 & 0.0371 & 0.0512 & 0.0361 & 0.0413 & 0.0465 & 0.0612 & 0.0504 \\
& 50 & 0.0382 & 0.0452 & 0.0357 & 0.0424 & 0.0447 & 0.0517 & 0.0471 \\
& 100 & 0.0401 & 0.0449 & 0.0402 & 0.0439 & 0.0450 & 0.0462 & 0.0430 \\
\hline 2 & 10 & 0.0295 & 0.0542 & 0.0178 & 0.0149 & 0.0245 & 0.0587 & 0.0246 \\
& 30 & 0.0225 & 0.0337 & 0.0196 & 0.0218 & 0.0254 & 0.0402 & 0.0300 \\
& 50 & 0.0245 & 0.0289 & 0.0228 & 0.0253 & 0.0278 & 0.0354 & 0.0305 \\
& 100 & 0.0251 & 0.0269 & 0.0231 & 0.0261 & 0.0273 & 0.0313 & 0.0278 \\
\hline 4 & 10 & 0.0231 & 0.0250 & 0.0063 & 0.0034 & 0.0072 & 0.0288 & 0.0100 \\
& 30 & 0.0096 & 0.0115 & 0.0043 & 0.0049 & 0.0056 & 0.0148 & 0.0088 \\
& 50 & 0.0084 & 0.0096 & 0.0063 & 0.0064 & 0.0071 & 0.0117 & 0.0086 \\
& 100 & 0.0092 & 0.0099 & 0.0080 & 0.0091 & 0.0101 & 0.0112 & 0.0100 \\
\hline
\end{tabular}

Table 3.5: Estimated levels of a nominal $\alpha=0.05$ test for Student's $t$-distribution $\left(T_{n}\right)$ with $n-2$ d.f., Limiting normal distribution $\left(Z_{n}\right)$ and Satterthwaite's approximation $\left(S_{n}\right)$ of $\hat{\Delta}_{1}$, Student's $t$-distribution $\left(T_{n}^{S R S}\right)$ with $2 n-2$ d.f. and the Limiting normal distribution $\left(Z_{n}^{S R S}\right)$ of $\hat{\Delta}$, the Limiting normal distribution $\left(Z_{n}^{3}\right)$ and Student's $t$ distribution $\left(T_{n}^{3}\right)$ with $n-1$ d.f. of $\hat{\Delta}_{3}$. Simulations are based on 10,000 replicates, $\sigma_{1}=\sigma_{2}=1$. The underlying distribution for $\epsilon$ is lognormal with mean of $\log (\epsilon)$ set to 0 and standard deviation of $\log (\epsilon)$ set to $0.5,1,2$, and 4 . Standard errors range from 0.001 to 0.003 . 


\begin{tabular}{ccccccccc}
\hline d.f. & $n$ & $T_{n}\left(\hat{\Delta}_{1}\right)$ & $Z_{n}\left(\hat{\Delta}_{1}\right)$ & $S_{n}\left(\hat{\Delta}_{1}\right)$ & $T_{n}^{S R S}(\hat{\Delta})$ & $Z_{n}^{S R S}(\hat{\Delta})$ & $Z_{n}^{3}\left(\hat{\Delta}_{3}\right)$ & $T_{n}^{3}\left(\hat{\Delta}_{3}\right)$ \\
\hline 5 & 10 & 0.0447 & 0.1127 & 0.0559 & 0.0476 & 0.0616 & 0.0820 & 0.0444 \\
& 30 & 0.0470 & 0.0588 & 0.0449 & 0.0472 & 0.0522 & 0.0628 & 0.0514 \\
& 50 & 0.0483 & 0.0527 & 0.0457 & 0.0476 & 0.0504 & 0.0517 & 0.0460 \\
& 100 & 0.0474 & 0.0500 & 0.0472 & 0.0490 & 0.0504 & 0.0532 & 0.0489 \\
\hline 10 & 10 & 0.0492 & 0.1158 & 0.0637 & 0.0499 & 0.0682 & 0.0824 & 0.0433 \\
& 30 & 0.0443 & 0.0585 & 0.0444 & 0.0469 & 0.0524 & 0.0582 & 0.0489 \\
& 50 & 0.0501 & 0.0584 & 0.0499 & 0.0519 & 0.0545 & 0.0586 & 0.0527 \\
& 100 & 0.0465 & 0.0488 & 0.0458 & 0.0531 & 0.0543 & 0.0543 & 0.0523 \\
\hline 20 & 10 & 0.0476 & 0.1174 & 0.0632 & 0.0526 & 0.0672 & 0.0806 & 0.0460 \\
& 30 & 0.0493 & 0.0622 & 0.0508 & 0.0495 & 0.0537 & 0.0580 & 0.0472 \\
& 50 & 0.0473 & 0.0557 & 0.0485 & 0.0492 & 0.0522 & 0.0545 & 0.0493 \\
& 100 & 0.0485 & 0.0506 & 0.0471 & 0.0524 & 0.0538 & 0.0558 & 0.0530 \\
\hline 40 & 10 & 0.0502 & 0.1095 & 0.0600 & 0.0452 & 0.0595 & 0.0684 & 0.0364 \\
& 30 & 0.0475 & 0.0585 & 0.0450 & 0.0479 & 0.0526 & 0.0571 & 0.0465 \\
& 50 & 0.0445 & 0.0508 & 0.0448 & 0.0468 & 0.0493 & 0.0525 & 0.0462 \\
& 100 & 0.0515 & 0.0544 & 0.0507 & 0.0512 & 0.0524 & 0.0520 & 0.0498 \\
\hline
\end{tabular}

Table 3.6: Estimated levels of a nominal $\alpha=0.05$ test for Student's $t$-distribution $\left(T_{n}\right)$ with $n-2$ d.f., Limiting normal distribution $\left(Z_{n}\right)$ and Satterthwaites approximation $\left(S_{n}\right)$ of $\hat{\Delta}_{1}$, Student's $t$-distribution $\left(T_{n}^{S R S}\right)$ with $2 n-2$ d.f. and the Limiting normal distribution $\left(Z_{n}^{S R S}\right)$ of $\hat{\Delta}$, and the Limiting normal distribution $\left(Z_{n}^{3}\right)$, Student's $t$ distribution $\left(T_{n}^{3}\right)$ with $n-1$ d.f. of $\hat{\Delta}_{3}$. Simulations are based on 10,000 replicates, $\sigma_{1}=\sigma_{2}=1$. The underlying distributions for $\epsilon$ are $\mathrm{t}$ with d.f. 5, 10, 20, and 40 . Standard errors range from 0.001 to 0.003 . 


\section{CHAPTER 4}

\section{PERMUTATION TESTS}

\subsection{Permutation Tests for the JPS Estimator $\hat{\Delta}_{1}$}

\subsubsection{Procedure}

The inferential procedures based on the limiting distribution of $\hat{\Delta}_{1}$ developed in the previous chapters depend on the underlying distributions of the populations. When $F_{X}$ and $G_{Z}$ are not normal, exact levels of the asymptotic tests proposed in Chapter 2 are no longer $\alpha$ although they tend to $\alpha$ as $n_{1} \rightarrow \infty$ and $\left(n-n_{1}\right) \rightarrow \infty$ using the asymptotic normality of $\hat{\Delta}_{1}$ developed in Chapter 2. This can be a problem when the sample size is small, especially for cases in which the populations are not close to normal. Therefore, we shall discuss an alternative approach, namely a permutation test, which yields the exact distribution of the estimators. The testing procedures and confidence limits we propose here are based on the randomization model and the randomization $t$-test procedure derived in Lehmann (1998).

Recall that for a Type 1 set, the control is assigned to a unit with rank 1 and the treatment is assigned to a unit with rank 2 , and vise versa for a Type 2 set. Let us take a one-sided test as an example. Suppose that we are interested in testing $H_{0}^{*}: \Delta=\mu_{1}-\mu_{2}=0$ against $H_{a}^{*}: \Delta=\mu_{1}-\mu_{2}<0$. Conditioning on the sets and 
ranks observed, let $\left(X_{1}, \cdots, X_{n_{1}}\right)$ and $\left(Z_{n_{1}+1}, \ldots, Z_{n}\right)$ denote the differences between the responses of the control and the treatment for the $n_{1}$ Type 1 sets and $n-n_{1}$ Type 2 sets, respectively. Moreover, under Model (2.2), we have (2.8), i.e., $\mu_{\epsilon[1]}=-\mu_{\epsilon[2]}$. This, together with (2.5) and (2.6), implies

$$
\begin{aligned}
& E\left(X_{i}\right)=\left(\mu_{1}-\mu_{2}\right)+\left(\sigma_{1}+\sigma_{2}\right) \mu_{\epsilon[1]}, \\
& E\left(Z_{j}\right)=\left(\mu_{1}-\mu_{2}\right)-\left(\sigma_{1}+\sigma_{2}\right) \mu_{\epsilon[1]}
\end{aligned}
$$

for $i=1, \ldots, n_{1}$ and $j=n_{1}, \ldots, n$.

To motivate our testing procedure, we first consider the case when $H_{0}^{*}$ is true. In that case, $\mu_{1}=\mu_{2}$ implies that there is no difference between the treatment group and the control group in terms of the treatment effect. Thus, $E\left(X_{i}\right)=\left(\sigma_{1}+\sigma_{2}\right) \mu_{\epsilon[1]}$ and $E\left(Z_{j}\right)=-\left(\sigma_{1}+\sigma_{2}\right) \mu_{\epsilon[1]}$. Therefore, we have $E\left(X_{i}\right)=E\left(-Z_{j}\right)$.

To have $\left(X_{1}, \ldots, X_{n_{1}},-Z_{n_{1}+1}, \ldots,-Z_{n}\right)$ identically distributed under the null hypothesis $H_{0}^{*}$, we need some additional assumptions. The following two cases are considered:

- Case 1: $\sigma_{1}=\sigma_{2}=\sigma$;

- Case 2: $\left(\epsilon_{[1]}, \epsilon_{[2]}\right)$ is equal in distribution to $\left(-\epsilon_{[2]},-\epsilon_{[1]}\right)$.

Recall that under Model (2.2), we have

$$
\begin{aligned}
& X_{i}=\mu_{1}-\mu_{2}+\sigma_{1} \epsilon_{[1] i}-\sigma_{2} \epsilon_{[2] i} \\
& Z_{j}=\mu_{1}-\mu_{2}+\sigma_{1} \epsilon_{[2] j}-\sigma_{2} \epsilon_{[1] j} .
\end{aligned}
$$

When $H_{0}^{*}$ is true, the above equations reduce to

$$
\begin{aligned}
& X_{i}=\sigma_{1} \epsilon_{[1] i}-\sigma_{2} \epsilon_{[2] i} \\
& Z_{j}=\sigma_{1} \epsilon_{[2] j}-\sigma_{2} \epsilon_{[1] j} .
\end{aligned}
$$


Under the equal variance assumption in Case 1, the following results are readily obtained:

$$
\begin{aligned}
& X_{i}=\sigma\left(\epsilon_{[1] i}-\epsilon_{[2] i}\right) \\
& Z_{j}=\sigma\left(\epsilon_{[2] j}-\epsilon_{[1] j}\right) .
\end{aligned}
$$

Thus, $X_{i}$ and $-Z_{j}$ are identically distributed.

Based on the assumption in Case 2 , we have $Z_{j}$ equal to $\sigma_{2} \epsilon_{[2] j}-\sigma_{1} \epsilon_{[1] j}$ in distribution. Therefore, $X_{i}$ and $-Z_{j}$ have identical distributions when Case 2 holds. One situation where Case 2 is satisfied is when the underlying distribution of the error term $\epsilon$ in Model (2.1) is symmetric and ranking is perfect. The situation can also be extended to some cases of imperfect ranking. One example is when rankings are made under Dell and Clutter's model (Dell and Clutter, 1972), where units are ranked based on estimates of the true random errors. In this model, $u_{i}=\epsilon_{i}+w_{i}$, where $\left(\epsilon_{i}, w_{i}\right) ; i=1,2, \cdots, n$, are all mutually independent, $\epsilon_{i}$ is $N(0,1)$, and the random noise $w_{i}$ is $N\left(0, \tau^{2}\right)$. To show the condition in Case 2 is satisfied here, we note that

$$
\begin{aligned}
& P\left(\epsilon_{[1]} \leq v, \epsilon_{[2]} \leq t\right) \\
= & P\left(\epsilon_{1} \leq v, \epsilon_{2} \leq t, u_{1} \leq u_{2}\right)+P\left(\epsilon_{2} \leq v, \epsilon_{1} \leq t, u_{1}>u_{2}\right) \\
= & P\left(-\epsilon_{1} \leq v,-\epsilon_{2} \leq t,-u_{1} \leq-u_{2}\right)+P\left(-\epsilon_{2} \leq v,-\epsilon_{1} \leq t,-u_{1}>-u_{2}\right) \\
= & P\left(-\epsilon_{1} \leq v,-\epsilon_{2} \leq t, u_{1} \geq u_{2}\right)+P\left(-\epsilon_{2} \leq v,-\epsilon_{1} \leq t, u_{1}<u_{2}\right) \\
= & P\left(-\epsilon_{[2]} \leq v,-\epsilon_{[1]} \leq t\right) .
\end{aligned}
$$

Therefore, the judgement ranked order statistics $\left(\epsilon_{[1]}, \epsilon_{[2]}\right)$ have the same distribution as $\left(-\epsilon_{[2]},-\epsilon_{[1]}\right)$ under Dell and Clutter's model, even when the rankings are not perfect. 
In more general cases where neither Case 1 nor 2 holds, $X_{i}$ and $-Z_{j}$ may not be identically distributed under the null hypothesis. One such example can be the situation when the underlying distribution of $\epsilon$ is lognormal with $\sigma_{1} \neq \sigma_{2}$, and the ranking is perfect or under Dell and Clutter's model. The level of the permutation test might drift a little bit.

To discuss our permutation test procedure, we assume the components of

$$
\left(a_{1}, \ldots, a_{n_{1}}, a_{n_{1}+1}, \cdots, a_{n}\right)=\left(X_{1}, \ldots, X_{n_{1}},-Z_{n_{1}+1}, \ldots,-Z_{n}\right)
$$

are independently and identically distributed under null hypothesis. Then, our permutation test procedure can be summarized as follows:

1. Under the null hypothesis $H_{0}^{*}: \mu_{1}-\mu_{2}=0, a_{1}, a_{2}, \cdots, a_{n}$ are identically distributed regardless of which group each set is assigned to. Thus $a_{j} ; j=1, \ldots, n$, can be considered to be fixed and attached to the $n$ sets. The only random feature is the assignment of $n_{1}$ of these sets to be Type 1 and their $a$ values becoming $X^{\prime} s$.

2. Randomly assign $n_{1}$ of the sets to be Type 1 and the other $n-n_{1}$ sets to be Type 2. The assignments are made in such a way that all possible $\left(\begin{array}{l}n \\ n_{1}\end{array}\right)$ assignments are equally likely. Therefore,

$$
P\left(X_{1}^{*}=a_{i_{1}}, \cdots, X_{n_{1}}^{*}=a_{i_{n_{1}}}\right)=\frac{1}{\left(\begin{array}{c}
n \\
n_{1}
\end{array}\right)}
$$

for each choice of the $n_{1}$ subscripts $1 \leq i_{1}<i_{2}<\cdots<i_{n_{1}} \leq n$.

3. Let $S_{n}$ denote the set of $n_{1}$ subscripts assigned to be Type 1 . Then we can compute the estimator based on each permuted sample as

$$
\hat{\Delta}_{1}^{*}=\frac{1}{2}\left(\frac{1}{n_{1}} \sum_{j \in S_{n}} a_{j}-\frac{1}{n-n_{1}} \sum_{j \in S_{n}^{c}} a_{j}\right) .
$$


Notice that our estimator $\hat{\Delta}_{1}$ is just one realization of the permutation model. There are $\left(\begin{array}{c}n \\ n_{1}\end{array}\right)$ possible values for $\hat{\Delta}_{1}^{*}$ and each has probability $1 /\left(\begin{array}{c}n \\ n_{1}\end{array}\right)$. This randomization distribution is the distribution of $\hat{\Delta}_{1}^{*}$ under $H_{0}^{*}$ and will be used as the basis for the test of $H_{0}^{*}$ against $H_{a}^{*}$.

4. If $H_{a}^{*}$ is true, then the obtained $\hat{\Delta}_{1}$ tends to be small compared to the reference distribution under $H_{0}^{*}$. Let $r /\left(\begin{array}{c}n \\ n_{1}\end{array}\right)=\alpha$ and let $\hat{\Delta}_{1[1]}^{*} \leq \hat{\Delta}_{1[2]}^{*} \leq \ldots \leq \hat{\Delta}_{1\left[\left(\begin{array}{c}n \\ n_{1}\end{array}\right)\right]}^{*}$ denote the $\left(\begin{array}{c}n \\ n_{1}\end{array}\right)$ permutation test statistics. Then a level $\alpha$ test rejects $H_{0}^{*}$ if $\hat{\Delta}_{1}$ is among the $r$ smallest values of $\hat{\Delta}_{1}^{*}$, i.e., when

$$
\hat{\Delta}_{1} \leq K\left(a_{1}, \cdots, a_{n}\right)=\hat{\Delta}_{1[r]}^{*}
$$

where $K\left(a_{1}, \cdots, a_{n}\right)$ is the critical value.

The distribution of $\hat{\Delta}_{1}^{*}$ is discrete, conditioning on the sets and ranks observed. If all of the potential values of $X_{i}$ and $-Z_{j}$ differ, the distribution is uniform. Consequently, the above test procedure may not be able to cover all possible values of level $\alpha$. In fact, it can only cover $\left(\begin{array}{c}n \\ n_{1}\end{array}\right)$ choices. These values include $1 /\left(\begin{array}{c}n \\ n_{1}\end{array}\right), 2 /\left(\begin{array}{c}n \\ n_{1}\end{array}\right), \ldots, 1$. If a particular $\alpha$ other than these values is of interest, a randomized testing procedure can be used to obtain a permutation test with exact level $\alpha$. Assume $r /\left(\begin{array}{c}n \\ n_{1}\end{array}\right)<\alpha<$ $(r+1) /\left(\begin{array}{c}n \\ n_{1}\end{array}\right)$, then the null hypothesis $H_{0}^{*}$ will be rejected (1) if $\hat{\Delta}_{1} \leq \hat{\Delta}_{1[r]}$ with probability 1, and (2) if $\hat{\Delta}_{1[r]}<\hat{\Delta}_{1} \leq \hat{\Delta}_{1[r+1]}$ with probability $\left(\alpha-\frac{r}{\left(\begin{array}{c}n \\ n_{1}\end{array}\right)}\right)\left(\begin{array}{c}n \\ n_{1}\end{array}\right)$. To show that this randomized test procedure has level $\alpha$, we note that $P\left(\hat{\Delta}_{1} \leq \hat{\Delta}_{1[r]}\right)=r /\left(\begin{array}{c}n \\ n_{1}\end{array}\right)$ and $P\left(\hat{\Delta}_{1[r]}<\hat{\Delta}_{1} \leq \hat{\Delta}_{1[r+1]}\right)=1 /\left(\begin{array}{c}n \\ n_{1}\end{array}\right)$. Thus the test level is $\frac{r}{\left(\begin{array}{c}n \\ n_{1}\end{array}\right)}+\left(\alpha-\frac{r}{\left(\begin{array}{c}n \\ n_{1}\end{array}\right)}\right)\left(\begin{array}{c}n \\ n_{1}\end{array}\right) \frac{1}{\left(\begin{array}{c}n \\ n_{1}\end{array}\right)}=\alpha$.

So far, we have derived a permutation test procedure for one-sided tests. The idea can be extended for a two-sided test with $H_{0}^{*}: \Delta=\mu_{1}-\mu_{2}=0$ against $H_{a}^{*}: \Delta=\mu_{1}-\mu_{2} \neq 0$. Let $r /\left(\begin{array}{c}n \\ n_{1}\end{array}\right)=\alpha / 2$ and $\hat{\Delta}_{1[r]}^{*}$ denote the $r$-th order statistic 
among the $\left(\begin{array}{c}n \\ n_{1}\end{array}\right)$ permutation test statistics. A level $\alpha$ two-sided test rejects $H_{0}^{*}$ if $\hat{\Delta}_{1}$ is among either the $r$ smallest values or the $r$ largest values of $\hat{\Delta}_{1}^{*}$. Therefore, the rejection region for the two-sided test is

$$
\hat{\Delta}_{1} \leq K_{L}\left(a_{1}, \cdots, a_{n}\right)=\hat{\Delta}_{1[r]}^{*}
$$

or

$$
\hat{\Delta}_{1} \geq K_{U}\left(a_{1}, \cdots, a_{n}\right)=\hat{\Delta}_{1\left[\left(\begin{array}{c}
n \\
n_{1}
\end{array}\right)-r+1\right]}^{*}
$$

When the integer $r$ cannot be found to get a exact level $\alpha$, a randomized testing procedure analogous to that in the case of one-sided tests can be obtained.

\subsubsection{Properties}

In Section 4.1.1, we developed a permutation test procedure with level $\alpha$ for $H_{0}^{*}$ against $H_{a}^{*}$ based on the test statistic $\hat{\Delta}_{1}$. The rejection region of the test is given in (4.4). In fact, we can also develop similar permutation test procedures based on $\sum_{i=1}^{n_{1}} X_{i}$ and $T_{n}$

To apply the permutation procedure to $\sum_{i=1}^{n_{1}} X_{i}$, we first obtain all the values of $\sum_{i=1}^{n_{1}} X_{i}^{*}$, where $X_{1}^{*}, \ldots, X_{n_{1}}^{*}$ can be obtained by randomly selecting $n_{1}$ values without replacement from $\left(a_{1}, \ldots, a_{n}\right)$. Then we let $S_{X[1]} \leq S_{X[2]} \leq \ldots \leq S_{X\left[\left(\begin{array}{c}n \\ n_{1}\end{array}\right)\right]}$ denote all possible $\sum_{i=1}^{n_{1}} X_{i}^{*}$ based on $\left(\begin{array}{c}n \\ n_{1}\end{array}\right)$ permutations of $X_{1}^{*}, \ldots, X_{n_{1}}^{*}$. A rejection region for a level $\alpha$ hypothesis test for $H_{0}^{*}$ versus $H_{a}^{*}$ based on $\sum_{i=1}^{n_{1}} X_{i}$ can then be derived as

$$
\sum_{i=1}^{n_{1}} X_{i} \leq K^{s}\left(a_{1}, \cdots, a_{n}\right)=S_{X[r]}
$$

where $K^{s}\left(a_{1}, \cdots, a_{n}\right)$ represents the critical value for $\sum_{i=1}^{n_{1}} X_{i}$.

One can also apply the permutation procedure to the standardized version of $\sum_{i=1}^{n_{1}} X_{i}, V_{n}=\frac{\bar{X}_{n_{1}}-E\left(\bar{X}_{n_{1}}\right)}{\sqrt{\operatorname{Var}\left(\bar{X}_{n_{1}}\right)}}$. According to the permutation procedure, $X_{1}^{*}, \cdots, X_{n_{1}}^{*}$ 
is a simple random sample from a finite population $a_{1}, \cdots, a_{n}$. The sample mean $\bar{X}_{n_{1}}=\frac{1}{n_{1}} \sum_{i=1}^{n_{1}} X_{i}$ has mean and variance

$$
E\left(\bar{X}_{n_{1}}\right)=\frac{1}{n} \sum_{j=1}^{n} a_{j}=\bar{a}
$$

and

$$
\operatorname{Var}\left(\bar{X}_{n_{1}}\right)=\frac{n-n_{1}}{n_{1}(n-1) n} \sum_{j=1}^{n}\left(a_{j}-\bar{a}\right)^{2} .
$$

Since the permutation procedure is conditional on $\left(a_{1}, \ldots, a_{n}\right), E\left(\bar{X}_{n_{1}}\right)$ and $\operatorname{Var}\left(\bar{X}_{n_{1}}\right)$ are constants. Therefore, it is obvious that the permutation tests based on $\sum_{i=1}^{n_{1}} X_{i}$ and $V_{n}$ are equivalent.

We can also develop a similar permutation test procedure based on

$$
T_{n}=\frac{\hat{\Delta}_{1}}{\sqrt{\frac{1}{4}\left(\frac{1}{n_{1}}+\frac{1}{\left(n-n_{1}\right)}\right) S_{p}^{2}}} .
$$

To this end, we need to obtain all values of $T_{n}^{*}$, each corresponding to one permuted sample. Let $T_{n[1]}^{*} \leq T_{n[2]}^{*} \leq \ldots \leq T_{n\left[\left(\begin{array}{c}n \\ n_{1}\end{array}\right)\right]}^{*}$ denote all possible $T_{n}^{*}$ based on $\left(\begin{array}{c}n \\ n_{1}\end{array}\right)$ permutations. A rejection region for a level $\alpha$ hypothesis test for $H_{0}^{*}$ versus $H_{a}^{*}$ based on $T_{n}$ can then be derived as

$$
T_{n} \leq K^{t}\left(a_{1}, \cdots, a_{n}\right)=T_{n[r]}^{*}
$$

where $K^{t}\left(a_{1}, \cdots, a_{n}\right)$ represents the critical value for $T_{n}$.

Next, we investigate connections among the proposed permutation procedures. The following theorem states equivalence of the four permutation tests.

Theorem 5 For a hypothesis test $H_{0}^{*}: \mu_{1}-\mu_{2}=\Delta_{0}=0$ versus $H_{a}^{*}: \mu_{1}-\mu_{2}<0$, permutation tests based on $\hat{\Delta}_{1}, \sum_{i=1}^{n_{1}} X_{i}, V_{n}$ and $T_{n}$ are equivalent. 
Proof of Theorem 5: We have shown the equivalence of permutation tests based on $\sum_{i=1}^{n_{1}} X_{i}$ and $V_{n}$. To prove the theorem, it suffices for us to first show equivalence of permutation tests based on $\hat{\Delta}_{1}$ and $\sum_{i=1}^{n_{1}} X_{i}$ and then show equivalence of permutation tests based on $\hat{\Delta}_{1}$ and $T_{n}$.

Notice that

$$
\begin{aligned}
2 \frac{n-n_{1}}{n} \hat{\Delta}_{1} & =\frac{n-n_{1}}{n}(\bar{X}+\bar{Z}) \\
& =\frac{1}{n}\left(\left(n-n_{1}\right) \bar{X}+\left(n-n_{1}\right) \bar{Z}\right) \\
& =\frac{1}{n}\left[n \bar{X}-\left(n_{1} \bar{X}-\left(n-n_{1}\right) \bar{Z}\right)\right] \\
& =\frac{1}{n}\left[n \bar{X}-\sum_{j=1}^{n} a_{j}\right] .
\end{aligned}
$$

Therefore, the following result is readily obtained

$$
2 \frac{n-n_{1}}{n} \hat{\Delta}_{1}=\frac{1}{n_{1}} \sum_{i=1}^{n_{1}} X_{i}-\frac{1}{n} \sum_{j=1}^{n} a_{j} .
$$

Since $\sum_{j=1}^{n} a_{j}$ is a constant for all permutations, a hypothesis test based on $\sum_{i=1}^{n_{1}} X_{i}$ is equivalent to the test based on $\hat{\Delta}_{1}$.

Next, note that by (4.7) and (4.11), we have

$$
\bar{X}_{n_{1}}-E\left(\bar{X}_{n_{1}}\right)=2 \frac{n-n_{1}}{n} \hat{\Delta}_{1}=\frac{n-n_{1}}{n}\left(\bar{X}_{n_{1}}+\bar{Z}_{n-n_{1}}\right) .
$$

By some simple calculation, we have

$$
\sum_{j=1}^{n}\left(a_{j}-a .\right)^{2}=\sum_{i=1}^{n_{1}}\left(X_{i}-\bar{X}_{n_{1}}\right)^{2}+\sum_{j=n_{1}+1}^{n}\left(Z_{j}-\bar{Z}_{n-n_{1}}\right)^{2}+\frac{n_{1}\left(n-n_{1}\right)}{n}\left(\bar{X}_{n_{1}}+\bar{Z}_{n-n_{1}}\right)^{2} .
$$

Plugging the above equation into (4.8), the variance of $\bar{X}_{n_{1}}$ can be expressed in terms of $\bar{X}_{n_{1}}$ and $\bar{Z}_{n-n_{1}}$ as

$$
\begin{aligned}
\operatorname{Var}\left(\bar{X}_{n_{1}}\right) & =\frac{n-n_{1}}{n_{1}(n-1) n}\left[\sum_{i=1}^{n_{1}}\left(X_{i}-\bar{X}_{n_{1}}\right)^{2}+\sum_{j=n_{1}+1}^{n}\left(Z_{j}-\bar{Z}_{n-n_{1}}\right)^{2}\right] \\
& +\frac{\left(n-n_{1}\right)^{2}}{n^{2}(n-1)}\left(\bar{X}_{n_{1}}+\bar{Z}_{n-n_{1}}\right)^{2} .
\end{aligned}
$$


Then the test statistic $T_{n}$ can be expressed as

$$
\begin{aligned}
& T_{n}=\frac{\hat{\Delta}_{1}}{\sqrt{\frac{1}{4}\left(\frac{1}{n_{1}}+\frac{1}{\left(n-n_{1}\right)}\right) S_{p}^{2}}} \\
& =\frac{\left(\bar{X}_{n_{1}}+\bar{Z}_{n-n_{1}}\right)}{\sqrt{\left(\frac{1}{n_{1}}+\frac{1}{n-n_{1}}\right) \frac{1}{n-2} \sum_{i=1}^{n_{1}}\left(X_{i}-\bar{X}_{n_{1}}\right)^{2}+\sum_{j=n_{1}+1}^{n}\left(Z_{j}-\bar{Z}_{n-n_{1}}\right)^{2}}} \\
& =\frac{1}{\sqrt{\left(\frac{1}{n_{1}}+\frac{1}{n-n_{1}}\right) \frac{1}{n-2} \frac{\sum_{i=1}^{n_{1}}\left(X_{i}-\bar{X}_{n_{1}}\right)^{2}+\sum_{j=n_{1}+1}^{n}\left(Z_{j}-\bar{Z}_{n-n_{1}}\right)^{2}}{\left(\bar{X}_{n_{1}}+\bar{Z}_{n-n_{1}}\right)^{2}}}} .
\end{aligned}
$$

From (4.8) and (4.12), we can see that

$\sum_{i=1}^{n_{1}}\left(X_{i}-\bar{X}_{n_{1}}\right)^{2}+\sum_{j=n_{1}+1}^{n}\left(Z_{j}-\bar{Z}_{n-n_{1}}\right)^{2}=\sum_{j=1}^{n}\left(a_{j}-a .\right)^{2}-\frac{n_{1}\left(n-n_{1}\right)}{n}\left(\bar{X}_{n_{1}}+\bar{Z}_{n-n_{1}}\right)^{2}$.

Consider two JPS estimates $\hat{\Delta}_{1}^{1}$ and $\hat{\Delta}_{1}^{2}$ with corresponding pooled variance estimates $S_{p}^{1}$ and $S_{p}^{2}$. Their corresponding standardized estimators based on (4.9) are denoted as $T_{n}^{1}$ and $T_{n}^{2}$. Since $\sum_{j=1}^{n}\left(a_{j}-a .\right)^{2}$ is a constant for all permutations, we have $S_{p}^{1} \geq S_{p}^{2}$ if $\hat{\Delta}_{1}^{1} \leq \hat{\Delta}_{1}^{2}$. Thus, if $\hat{\Delta}_{1}^{1} \leq \hat{\Delta}_{1}^{2}$, then $T_{n}^{1} \leq T_{n}^{2}$. Hence, a hypothesis test based on $T_{n}$ is equivalent to the test based on $\hat{\Delta}_{1}$. This completes the proof of Theorem 5 .

To investigate asymptotic properties of the proposed permutation test, we first embed the given vector $\left(a_{1}, \cdots, a_{n}\right)$ in a sequence of such vectors: $\left\{\left(a_{n 1}, \cdots, a_{n n}\right), n=\right.$ $1,2, \cdots\}$. Theorem 6 describes some asymptotic behavior of the permutation test procedure for testing $H_{0}^{*}$ versus $H_{a}^{*}$.

Theorem 6 If $F_{X}$ and $G_{Z}$ have finite second moments, then given almost every sequence $\left\{\left(a_{n 1}, \cdots, a_{n n}\right), n=1,2, \cdots\right\}$, the sequence $\sqrt{n} \hat{\Delta}_{1}^{*}$ of size $\left(\begin{array}{c}n \\ n_{1}\end{array}\right)$ is asymptotically normal with mean zero. If $X_{i}$ and $-Z_{j}$ are i.i.d., the asymptotic variance is equal to $\operatorname{Var}(X)$.

Proof of Theorem 6: This theorem follows directly from Theorem 13.25 of van der Vaart (1998) by setting $f(u)=u / 2$ and $\lambda=1 / 2$. 
In summary, we have shown that the permutation tests based on $\hat{\Delta}_{1}, \sum_{i=1}^{n_{1}} X_{i}$, $V_{n}$, and $T_{n}$ are equivalent. We have also shown the asymptotic normality of the permutation test based on $\Delta_{1}$. The result is similar to the asymptotic test based on $T_{n}$. As a result, the proposed permutation procedure helps to remedy the inadequacy of the asymptotic test based on $T_{n}$ for the case of small sample sizes. However, as the sample sizes get large, both the permutation tests and the standard test based on $T_{n}$ have asymptotic normality and consequently using the asymptotic test based on $T_{n}$ alone will be sufficient.

Another possible permutation procedure is to assign each set to be Type 1 or Type 2 at random. In this case, $n_{1}$, the number of sets that have been assigned to be Type 1 , is not fixed. This permutation results in $2^{n}$ possible test statistics instead of $\left(\begin{array}{c}n \\ n_{1}\end{array}\right)$. We prefer to perform a permutation procedure based on the fixed $n_{1}$ and will not discuss this further.

\subsubsection{Extensions of The Permutation Tests}

The permutation testing procedure described so far uses the response variables $Y_{1}$ and $Y_{2}$. In particular, we utilize the fact that $\left(X_{1}, \ldots, X_{n_{1}},-Z_{n_{1}+1}, \ldots,-Z_{n}\right)$ are independently and identically distributed under the null hypothesis $H_{0}^{*}$. In fact, we can apply the idea of nonparametric tests, e.g. the Wilcoxon rank sum test. In particular, denote the ranks of $\left(X_{1}, \ldots, X_{n_{1}},-Z_{n_{1}+1}, \ldots,-Z_{n}\right)$ by $\left(r_{1}, \ldots, r_{n}\right)$. Then, we can compute the rank sum for the $n_{1}$ Type 1 sets $w=\sum_{j=1}^{n_{1}} r_{j}$. Clearly, we should reject $H_{0}^{*}$ when $w$ is small if $H_{a}^{*}=\mu_{1}-\mu_{2}<0$ is true. The critical value for the rejection region can be determined using the distribution of $w$ under $H_{0}^{*}$. This test is 
analogous to the two-sample Wilcoxon rank sum test and we do not discuss it further here.

\subsubsection{Confidence Intervals}

A confidence limit for $\Delta_{1}$ is often needed. It can be derived by inverting the onesided permutation test. For simplicity, assume $K /\left(\begin{array}{c}n \\ n_{1}\end{array}\right)=\alpha$ and $n_{1} \geq n-n_{1}$. Consider hypothesis testing with the null hypothesis $H_{0}^{* *}: \Delta=\Delta_{0}$ versus the alternative $H_{a}^{* *}$ : $\Delta<\Delta_{0}$. Then under the null hypothesis $H_{0}^{* *},(4.1)$ and (4.2) become

$$
\begin{aligned}
& E\left(X_{i}\right)=\Delta_{0}+\left(\sigma_{1}+\sigma_{2}\right) \mu_{\epsilon[1]}, \\
& E\left(Z_{j}\right)=\Delta_{0}-\left(\sigma_{1}+\sigma_{2}\right) \mu_{\epsilon[1]} .
\end{aligned}
$$

Clearly, $E\left(X_{i}-2 \Delta_{0}\right)=E\left(-Z_{j}\right)$ and $\left(b_{1}, \ldots, b_{n_{1}}, b_{n_{1}+1}, \cdots, b_{n}\right)=\left(X_{1}-2 \Delta_{0}, \ldots, X_{n_{1}}-\right.$ $\left.2 \Delta_{0},-Z_{n_{1}+1}, \ldots,-Z_{n}\right)$ are independently and identically distributed if $H_{0}^{* *}$ is true. As a result, we can perform permutations on $\left(b_{1}, \ldots, b_{n_{1}}, b_{n_{1}+1}, \cdots, b_{n}\right)$. Specifically, we randomly select $n_{1}$ samples from $\left(b_{1}, \ldots, b_{n_{1}}, b_{n_{1}+1}, \cdots, b_{n}\right)$ and form $n_{1}$ indices $\left(i_{1}, \cdots, i_{n_{1}}\right)$ for permuted Type 1 sets with probability $\frac{1}{\left(\begin{array}{c}n \\ n_{1}\end{array}\right)}$. Then the JPS estimator based on the permuted sample can be calculated as $\hat{\Delta}_{1}^{* *}=\frac{1}{2}\left[\frac{1}{n_{1}} \sum_{i=1}^{n_{1}}\left(b_{i}+2 \Delta_{0}\right)-\right.$ $\left.\frac{1}{\left(n-n_{1}\right)} \sum_{j=n_{1}+1}^{n} b_{j}\right]$. Therefore, in a level $\alpha$ test, we fail to reject the null hypothesis $H_{0}^{* *}$ against the alternative $H_{a}^{* *}$ if $\hat{\Delta}_{1}$ is among the $l=\left(\begin{array}{c}n \\ n_{1}\end{array}\right)-K$ largest $\hat{\Delta}_{1}^{* *}$ s obtained from the $\left(\begin{array}{c}n \\ n_{1}\end{array}\right)$ permutation samples. 
Notice that

$$
\begin{aligned}
2 \frac{n-n_{1}}{n} \hat{\Delta}_{1} & =\frac{n-n_{1}}{n}(\bar{X}+\bar{Z}) \\
& =\frac{1}{n}\left(\left(n-n_{1}\right) \bar{X}+\left(n-n_{1}\right) \bar{Z}\right) \\
& =\frac{1}{n}\left[n \bar{X}-\left(n_{1} \bar{X}-\left(n-n_{1}\right) \bar{Z}\right)\right] \\
& =\frac{1}{n}\left[n \bar{X}-2 n_{1} \Delta_{0}-\sum_{j=1}^{n} b_{j}\right] .
\end{aligned}
$$

Thus,

$$
\begin{aligned}
2 \frac{n-n_{1}}{n} \hat{\Delta}_{1}-2 \frac{n-n_{1}}{n} \Delta_{0} & =\frac{1}{n}\left[n \bar{X}-2 n_{1} \Delta_{0}-\sum_{j=1}^{n} b_{j}\right]-2 \frac{n-n_{1}}{n} \Delta_{0} \\
& =\bar{X}-2 \Delta_{0}-\frac{1}{n} \sum_{j=1}^{n} b_{j} .
\end{aligned}
$$

Moreover, for given $\Delta_{0}, \sum_{i=1}^{n} b_{j}$ is same for all permutation samples. Thus, equivalently, we do not reject $H_{0}^{* *}$ if $\sum_{j=1}^{n_{1}}\left(X_{j}-2 \Delta_{0}\right)$ is among the $l=\left(\begin{array}{c}n \\ n_{1}\end{array}\right)-K$ largest of all possible values in permutation. That is

$$
\sum_{j=1}^{n_{1}}\left(X_{j}-2 \Delta_{0}\right)>\left(-Z_{n_{1}+i_{1}}\right)+\cdots+\left(-Z_{n_{1}+i_{r}}\right)+\left[X_{j_{1}}+\cdots+X_{j_{n_{1}-r}}-\left(n_{1}-r\right) 2 \Delta_{0}\right]
$$

where $\left(i_{1}, \cdots, i_{r}, j_{1}, \cdots, j_{n_{1}-r}\right)$ is a permutation of the subscripts $\left(1,2, \cdots, n_{1}\right)$ and (4.17) should be true for at least $K$ such permutations. Notice that (4.17) is equivalent to

$$
X_{i_{1}}+\cdots+X_{i_{r}}>\left(-Z_{n_{1}+i_{1}}\right)+\cdots+\left(-Z_{n_{1}+i_{r}}\right)+2 r \Delta_{0}
$$

or

$$
\frac{1}{2}\left(\bar{X}_{i_{1}, \cdots, i_{r}}+\bar{Z}_{i_{1}, \cdots, i_{r}}\right)>\Delta_{0}
$$

As indicated by Lehmann (1998), the number of such averages is

$$
M=\sum_{r=1}^{n_{1}}\left(\begin{array}{c}
n_{1} \\
r
\end{array}\right)\left(\begin{array}{c}
n-n_{1} \\
r
\end{array}\right)=\left(\begin{array}{c}
n \\
n_{1}
\end{array}\right)-1
$$


with $r \geq 1$. Thus we fail to reject the null hypothesis $H_{0}^{* *}: \Delta=\Delta_{0}$ against the alternative $H_{a}^{* *}: \Delta<\Delta_{0}$ at level $\alpha$ if and only if at least $K$ of the $M$ averages are larger than $\Delta_{0}$, i.e., $\delta_{(l)}>\Delta_{0}$, where $\delta_{(1)}<\cdots<\delta_{(M)}$ are the ordered set of averages $\frac{1}{2}\left(\bar{X}_{i_{1}, \cdots, i_{r}}+\bar{Z}_{i_{1}, \cdots, i_{r}}\right)$ in (4.18). Here $\delta_{(l)}$ serves as the upper confidence bound for $\Delta$ with confidence coefficient $\gamma=1-\alpha$.

Now we have $M+1$ intervals

$$
\left(-\infty, \delta_{(1)}\right),\left(\delta_{(1)}, \delta_{(2)}\right), \cdots,\left(\delta_{(M-1)}, \delta_{(M)}\right),\left(\delta_{M}, \infty\right)
$$

each having probability $1 /\left(\begin{array}{c}n \\ n_{1}\end{array}\right)$ to contain the parameter $\Delta$. Thus, the two-sided confidence interval is obtained as $\left(\delta_{(K)}, \delta_{\left(\left(\begin{array}{c}n \\ n_{1}\end{array}\right)-K\right)}\right)$ with the confidence coefficient $\gamma=$ $(1-2 \alpha)$.

\subsection{A Permutation Test for The VRBE $\hat{\Delta}_{3}$}

A permutation test $H_{0}^{*}: \mu_{1}=\mu_{2}$ against $H_{0}^{*}: \mu_{1}<\mu_{2}$ can also be derived for the $\operatorname{VRBE} \hat{\Delta}_{3}$. However, in this case, all $n^{2}$ pairs are formed and ranked for a specific design. We will consider a different randomization model which allows us to find the exact distribution of the estimator. Here we assume there are $m_{1}$ Type 1 sets among the $n^{2}$ pairs with corresponding estimator VRBE $\hat{\Delta}_{3}$.

Under the null hypothesis $H_{0}^{*}$, the $2 n$ units from control and treatment are identically distributed. According to the randomization model, we can randomly assign $n$ out of $2 n$ units to treatment and the other $n$ to control in such a way that all the possible $\left(\begin{array}{c}2 n \\ n\end{array}\right)$ assignments are equally likely. For each assignment, one can find the corresponding $m_{1}^{*}$, which is the number of Type 1 sets among all the $n^{2}$ possible pairs. For the permutation procedure, we need to condition on $m_{1}$ in order to preserve the 
information in the original sample. Therefore, we only use those permutated samples for which $m_{1}^{*}=m_{1}$ and compute the corresponding estimator $\hat{\Delta}_{3}^{*}$.

We now describe the procedure in detail. Let

$$
\left(Y_{11}^{*}, \ldots, Y_{1 n}^{*}, Y_{21}^{*}, \ldots, Y_{2 n}^{*}\right)
$$

denote the permutated sample, where $\left(Y_{11}^{*}, \ldots, Y_{1 n}^{*}\right)$ and $\left(Y_{21}^{*}, \ldots, Y_{2 n}^{*}\right)$ correspond to the treatment group and control groups respectively. Suppose we can rank EU's using the extra information from 1 to $2 n$ and the corresponding ranks for this permuted sample are given by

$$
\left(R_{Y_{11}^{*}}, \ldots, R_{Y_{1 n}^{*}}, R_{Y_{21}^{*}}, \ldots, R_{Y_{2 n}^{*}}\right) .
$$

Then we can derive $m_{1}^{*}$ as $\sum_{j=1}^{n} \sum_{\ell=1}^{n} I\left(R_{Y_{1 j}^{*}} \leq R_{Y_{2 \ell}^{*}}\right)$. We only keep those samples with $m_{1}^{*}=m_{1}$. Assume there are $M$ such permuted samples. Then there are $M$ possible values for $\hat{\Delta}_{3}^{*}$ and each has probability $1 / M$. This permutation distribution is the distribution of $\hat{\Delta}_{3}^{*}$ under $H_{0}^{*}$ and will be used as the basis for the test of $H_{0}^{*}$ against $H_{a}^{*}$.

If $H_{a}^{*}$ is true, then the obtained $\hat{\Delta}_{3}$ tends to be small compared to that under $H_{0}^{*}$. Let $r / M=\alpha$ and denote the $M$ permutation test statistics as $\hat{\Delta}_{3[1]}^{*} \leq \hat{\Delta}_{3[2]}^{*} \leq \ldots \leq$ $\hat{\Delta}_{3[M]}^{*}$. Then a level $\alpha$ test rejects $H_{0}^{*}$ if $\hat{\Delta}_{3}$ is among the $r$ smallest values of $\hat{\Delta}_{3}^{*}$.

Similar to that of $\hat{\Delta}_{1}$, the above test procedure may not be able to cover all possible values of level $\alpha$. It can only cover $M$ choices: $1 / M, 2 / M, \ldots, 1$. If a particular $\alpha$ other than these values is of interest, a randomized testing procedure can be used to obtain a permutation test with exact level $\alpha$. Assume $r / M<\alpha<(r+1) / M$, then the null hypothesis $H_{0}^{*}$ will be rejected (1) if $\hat{\Delta}_{3} \leq \hat{\Delta}_{3[r]}$ with probability 1 , and (2) if $\hat{\Delta}_{3[r]}<\hat{\Delta}_{3} \leq \hat{\Delta}_{3[r+1]}$ with probability $\alpha M-r$. 


\subsection{Simulations}

Theorem 5 shows that the permutation tests based on $\hat{\Delta}_{1}, \sum_{i=1}^{n_{1}} X_{i}, V_{n}$, and $T_{n}$ are equivalent. Therefore, to illustrate the performance of our proposed permutation

tests, it is sufficient to use $\hat{\Delta}_{1}$ as the test statistic for our simulation study. We compare the permutation test based on $\hat{\Delta}_{1}$ with the asymptotic test based on $T_{n}$ proposed in Chapter 2 for the one-sided test $H_{0}^{*}: \Delta=\mu_{1}-\mu_{2}=0$ against $H_{a}^{*}: \Delta=\mu_{1}-\mu_{2}<0$. The same data generation mechanism as in Chapter 2 is adapted. Sample sizes chosen for this simulation study are 7, 8, 9, 10, 30, 50 and 100. We choose relatively small sample sizes as well as large sample sizes here for the following two purpose of illustration: (1) The permutation test is robust to various assumptions for the underlying distributions. Therefore we expect that it can deliver better performance than the asymptotic $t$-test for small sample sizes. (2). Theorem 6 says that the permutation test based on $\hat{\Delta}_{1}$ and the asymptotic test based on $T_{n}$ are asymptotically equivalent. Therefore, they are expected to deliver similar performance for large samples.

For smaller sample sizes including 7, 8,9 and 10, we calculate $\left(\begin{array}{c}n \\ n_{1}\end{array}\right) \hat{\Delta}_{1}^{*}$ s s to obtain the reference distribution for $\hat{\Delta}_{1}$. However, as $n$ increases, this number increases extremely fast and becomes too large to handle for sample sizes greater than 30 . In order to increase computational efficiency, for sample sizes 30, 50, and 100, we use an approximation of the exact distribution of $\hat{\Delta}_{1}$. In particular, we randomly choose $1000 \hat{\Delta}_{1}^{*}$ 's out of $\left(\begin{array}{c}n \\ n_{1}\end{array}\right)$ possible $\hat{\Delta}_{1}^{*}$ 's. The empirical levels and powers are calculated based on the approximate distribution of $\hat{\Delta}_{1}$.

Simulation results are reported in Tables 4.1-4.4 and Figures 4.1-4.8. The estimated levels of the two tests are listed in the tables and their estimated powers are plotted in the figures. In summary, we can conclude that 
- The estimated levels of the permutation test are closer to the actual level than the asymptotic test for relatively small sample sizes.

- The permutation test is more robust to skewness of the underlying distribution than the $t$-test.

- The permutation test delivers similar performance to the asymptotic $t$-test for large sample sizes.

Table 4.1 displays the estimated levels obtained from 10,000 replicates for the $t$-test with $n-2$ d.f. and the permutation test for $\hat{\Delta}_{1}$. The underlying distribution for $\epsilon_{i j}$ is $N(0,1)$. For the $t$-test, its estimated level can exceed the nominal level for small sample sizes. For example, in the case of perfect ranking, the estimated level is 0.0644 in contrast to the nominal level 0.05 when $n=7$. The permutation test, in contrast, preserves the level very well. In fact, almost all estimated levels are very close to the nominal level of 0.05 .

Under the same setting as in the previous paragraph, we also examine the powers of the corresponding tests. Figures 4.1-4.4 plot the estimated powers of the two tests with $\mu_{2}-\mu_{1}=0.1(0.1) 2$ and $n=7,10,30,50$, and 100 with ranking quality varying from perfect ranking $(\rho=1)$ to random ranking $(\rho=0)$. As we can see from the plots, the two tests have similar powers. It is not surprising to see that the $t$-test is slightly more powerful than the permutation test when the sample size is equal to 7. As the sample size increases to 10, the two tests almost have the same power. However, considering the larger levels of the $t$-test, we conclude that our permutation test is relatively conservative and almost as powerful as the $t$-test. 
Next we examine the robustness of the tests across different assumptions of the underlying distribution of $\epsilon_{i j}$. Table 4.2 presents the estimated levels for a nominal level $\alpha=0.05$ test based on 10,000 replicates when treatment variances are set to be 1 and 4 respectively. Table 4.3 lists the estimated levels for a nominal level $\alpha=0.05$ test when the underlying distribution of $\epsilon$ is $t$-distribution with d.f. being 5,10 , 20, and 40. Our results indicate that both tests are robust when the underlying distribution either has unequal variances for the two treatments or has thick tails.

To study the robustness of the permutation test to skewness, we choose the underlying distribution for $\epsilon_{i j}$ to be lognormal with mean of $\log (\epsilon)=0$ and standard deviation of $\log (\epsilon), \sigma$, to be $0.5,1,2,4$ respectively. As $\sigma$ gets larger, the distribution of $\epsilon_{i j}$ becomes more skewed. The estimated levels for both tests under perfect ranking are summarized in Table 4.4. Clearly, the $t$-test performs poorly in terms of level. With the nominal level 0.05 , the estimated levels for $t$-test can be as large as 0.0605 and as small as 0.0194. On the contrary, the proposed permutation test preserves levels very nicely. This supports the robustness of the permutation test.

We also plot the estimated powers of the two tests with lognormal random errors in Figures 4.5-4.8 using standard deviation of $\log (\epsilon) \sigma=0.5,1,2,4$ and $n=7,10,30,50$, and 100 under perfect ranking. For the purpose of illustration, we also vary the parameter $\Delta=\mu_{1}-\mu_{2}$. Specifically, when $\sigma$ is $0.5, \Delta=0.1(0.1) 2$. When $\sigma$ is changed to $0.5 a, \Delta=2^{a} / 20\left(2^{a} / 20\right) 2^{a}$. This implies $\Delta=0.8(0.8) 16$ if $\sigma$ is 2 . As shown in the plot, the permutation test becomes more powerful than the $t$-test when the underlying distribution is fairly skewed, for example when the standard deviation of $\log (\epsilon)$ is 2 or 4 . In short, the permutation test preserves the level well and can deliver larger powers for skewed distribution. 


\begin{tabular}{cccc}
\hline$\rho$ & $n$ & $T_{n}\left(\hat{\Delta}_{1}\right)$ & $\operatorname{Perm}\left(\hat{\Delta}_{1}\right)$ \\
\hline 1 & 7 & $0.0644(0.0025)$ & $0.0484(0.0021)$ \\
& 8 & $0.0574(0.0023)$ & $0.0478(0.0021)$ \\
& 9 & $0.0505(0.0022)$ & $0.0497(0.0022)$ \\
& 10 & $0.0482(0.0021)$ & $0.0495(0.0022)$ \\
& 30 & $0.0474(0.0021)$ & $0.0527(0.0022)$ \\
& 50 & $0.0501(0.0022)$ & $0.0489(0.0022)$ \\
& 100 & $0.0549(0.0023)$ & $0.0515(0.0022)$ \\
\hline 0.7 & 7 & $0.0520(0.0022)$ & $0.0489(0.0022)$ \\
& 8 & $0.0545(0.0023)$ & $0.0488(0.0022)$ \\
& 9 & $0.0532(0.0022)$ & $0.0493(0.0022)$ \\
& 10 & $0.0478(0.0021)$ & $0.0535(0.0023)$ \\
& 30 & $0.0503(0.0022)$ & $0.0514(0.0022)$ \\
& 50 & $0.0536(0.0023)$ & $0.0488(0.0022)$ \\
& 100 & $0.0467(0.0021)$ & $0.0505(0.0022)$ \\
\hline 0.5 & 7 & $0.0506(0.0022)$ & $0.0457(0.0021)$ \\
& 8 & $0.0544(0.0023)$ & $0.0504(0.0022)$ \\
& 9 & $0.0524(0.0022)$ & $0.0483(0.0021)$ \\
& 10 & $0.0475(0.0021)$ & $0.0494(0.0022)$ \\
& 30 & $0.0491(0.0022)$ & $0.0520(0.0022)$ \\
& 50 & $0.0552(0.0023)$ & $0.0474(0.0021)$ \\
& 100 & $0.0476(0.0021)$ & $0.0506(0.0022)$ \\
\hline 0 & 7 & $0.0494(0.0022)$ & $0.0452(0.0021)$ \\
& 8 & $0.0523(0.0022)$ & $0.0490(0.0022)$ \\
& 9 & $0.0527(0.0022)$ & $0.0494(0.0022)$ \\
& 10 & $0.0491(0.0022)$ & $0.0474(0.0021)$ \\
& 30 & $0.0496(0.0022)$ & $0.0496(0.0022)$ \\
& 50 & $0.0536(0.0023)$ & $0.0485(0.0021)$ \\
100 & $0.0468(0.0021)$ & $0.0506(0.0022)$ \\
\hline & & &
\end{tabular}

Table 4.1: Estimated levels of a nominal level $\alpha=0.05$ test based on Student's $t$-distribution $\left(T_{n}\right)$ with $n-2$ d.f. and the permutation test based on $\hat{\Delta}_{1}$. The simulation is based on 10,000 replicates. The underlying distribution of $\epsilon$ is $N(0,1)$, $\sigma_{1}=\sigma_{2}=1$. Standard errors are given in parentheses. 
perfect ranking with $n=7$

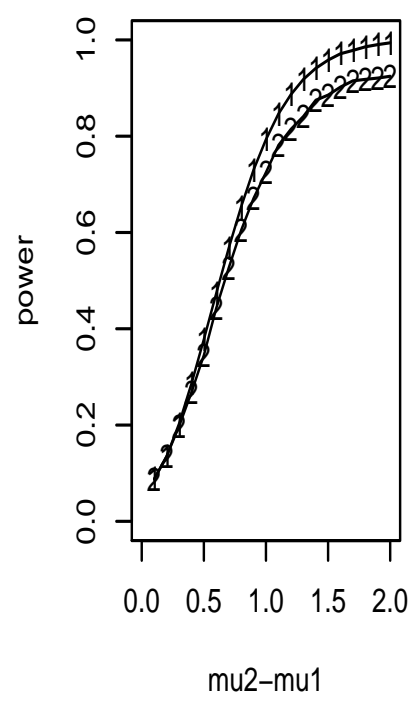

perfect ranking with $\mathrm{n}=50$

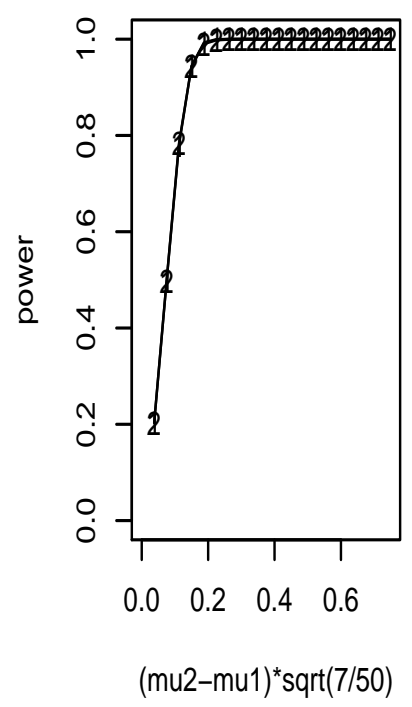

perfect ranking with $n=10$
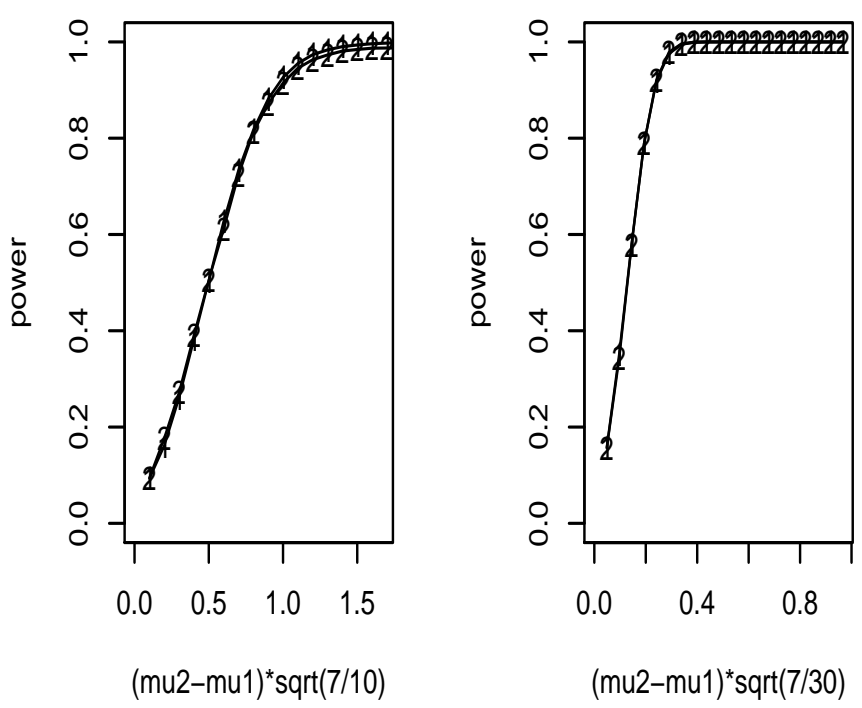

perfect ranking with $\mathrm{n}=30$

perfect ranking with $n=10 c$

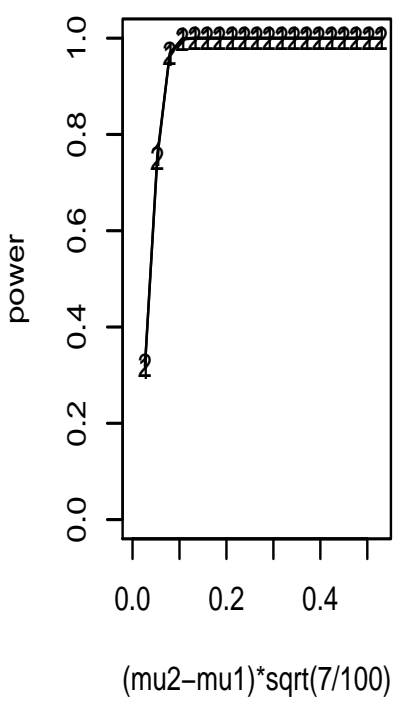

Figure 4.1: Empirical power of $T_{n}$ with $n-2$ d.f. with label 1 and permutation test based on $\hat{\Delta}_{1}$ with label 2 for perfect ranking. The simulation is based on 10,000 replicates. The underlying distribution of $\epsilon$ is $N(0,1), \sigma_{1}=\sigma_{2}=1$. 


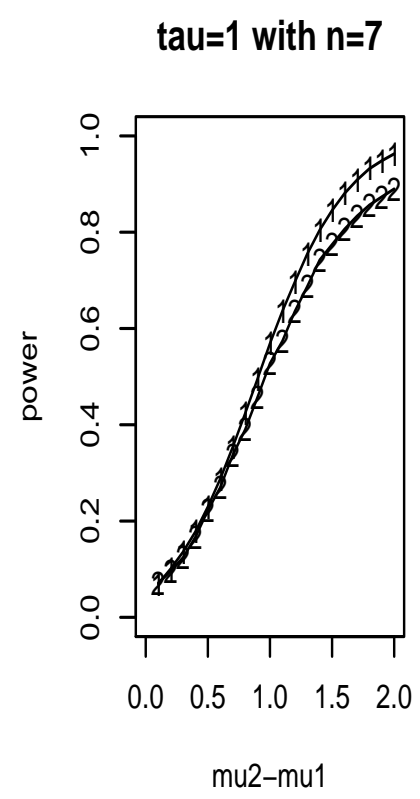

tau= 1 with $n=10$

tau= 1 with $n=30$
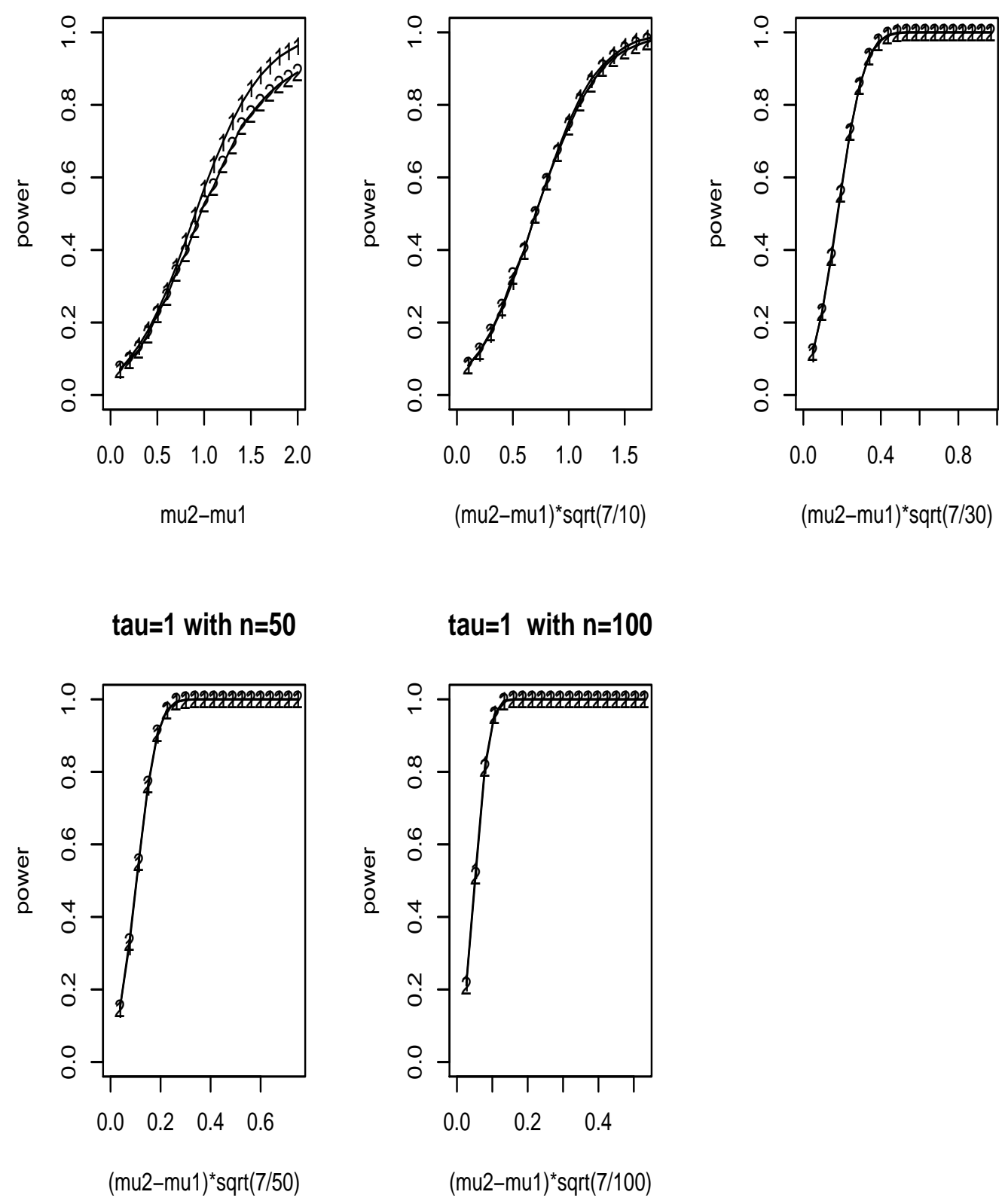

Figure 4.2: Empirical power of $T_{n}$ with $n-2$ d.f. with label 1 and permutation test based on $\hat{\Delta}_{1}$ with label 2 for $\tau=1$. The simulation is based on 10,000 replicates. The underlying distribution of $\epsilon$ is $N(0,1), \sigma_{1}=\sigma_{2}=1$. 
tau=sqrt(3) with $n=7$

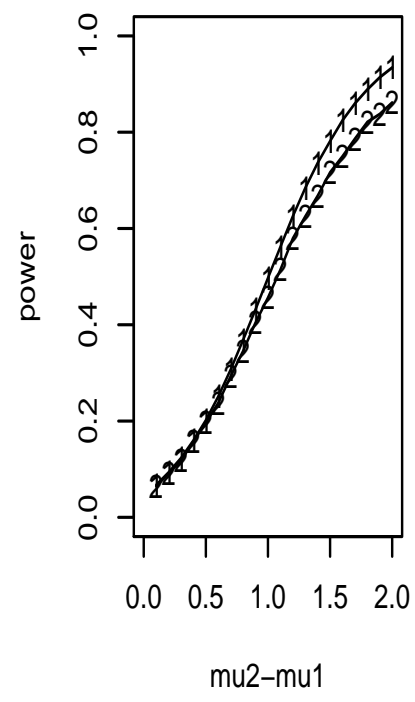

tau=sqrt(3) with $n=50$

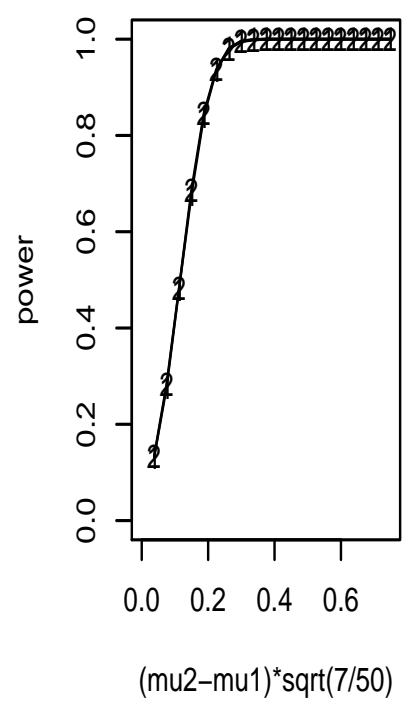

tau=sqrt(3) with $n=10$

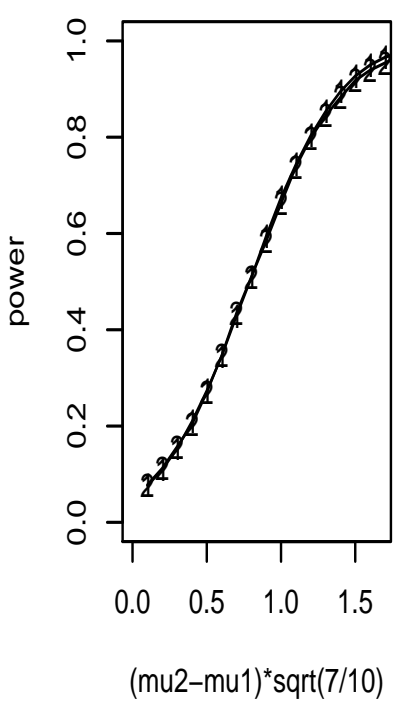

tau=sqrt(3) with $n=100$

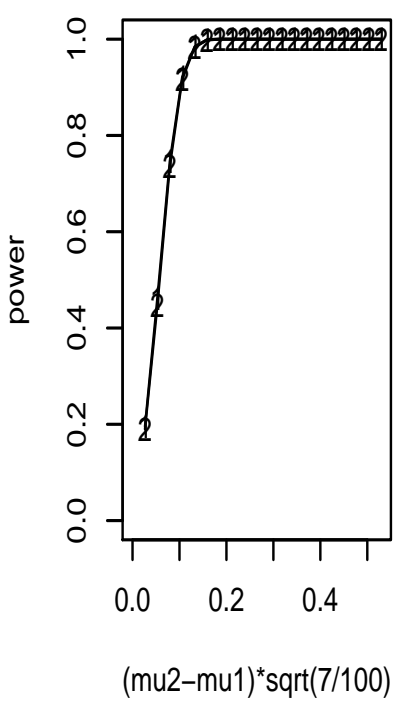

tau=sqrt(3) with $n=30$

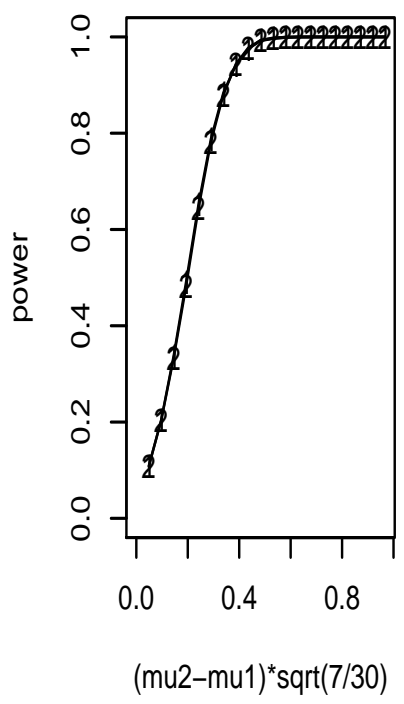

Figure 4.3: Empirical power of $T_{n}$ with $n-2$ d.f. with label 1 and permutation test based on $\hat{\Delta}_{1}$ with label 2 for $\tau=\sqrt{3}$. The simulation is based on 10,000 replicates. The underlying distribution of $\epsilon$ is $N(0,1), \sigma_{1}=\sigma_{2}=1$. 

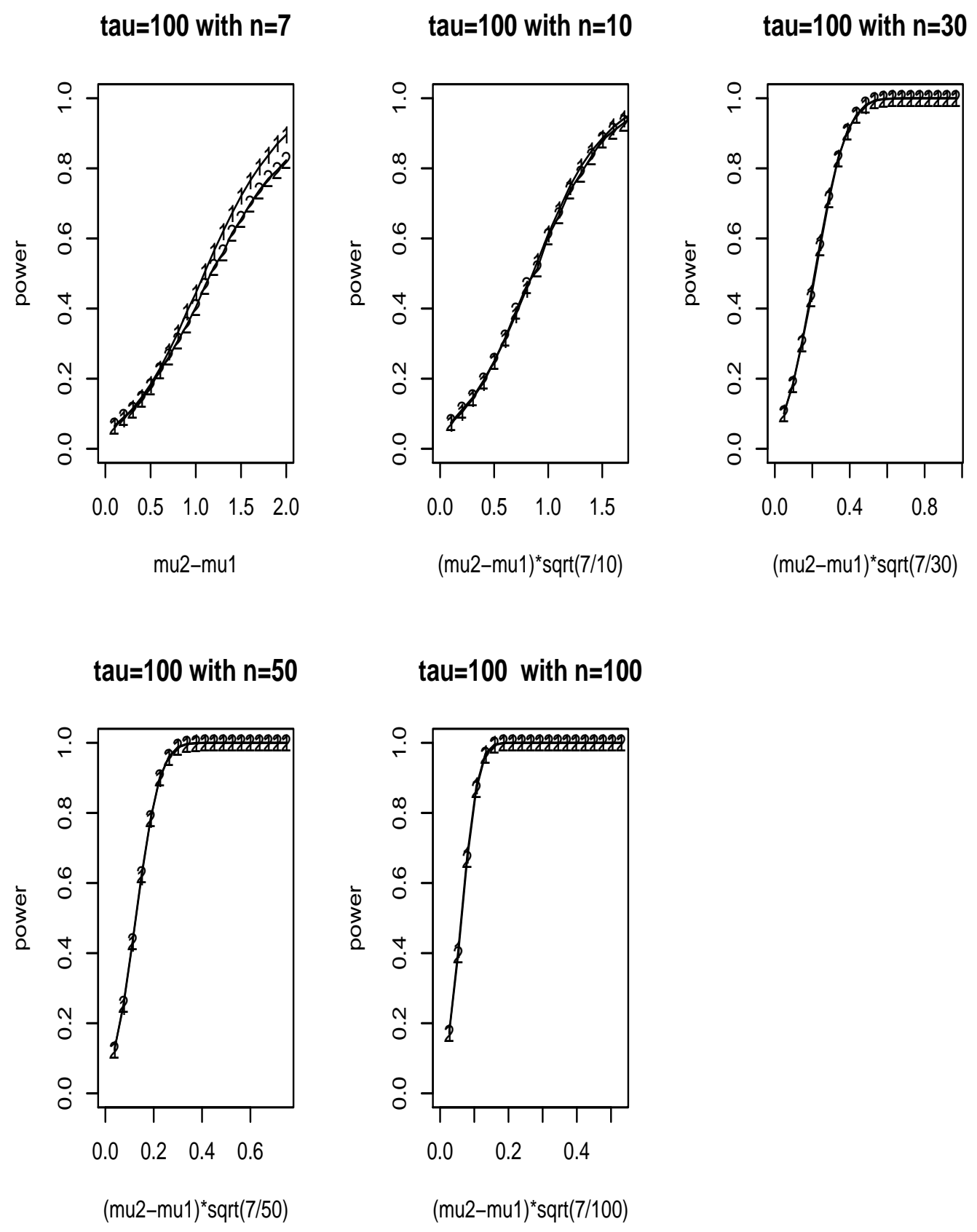

Figure 4.4: Empirical power of $T_{n}$ with $n-2$ d.f. with label 1 and permutation test based on $\hat{\Delta}_{1}$ with label 2 for $\tau=100$. The simulation is based on 10,000 replicates. The underlying distribution of $\epsilon$ is $N(0,1), \sigma_{1}=\sigma_{2}=1$. 


\begin{tabular}{cccc}
\hline$\sigma$ & $n$ & $T_{n}\left(\hat{\Delta}_{1}\right)$ & $\operatorname{Perm}\left(\hat{\Delta}_{1}\right)$ \\
\hline 1 & 7 & $0.0578(0.0023)$ & $0.0514(0.0022)$ \\
& 8 & $0.0556(0.0023)$ & $0.0515(0.0022)$ \\
& 9 & $0.0509(0.0022)$ & $0.0461(0.0021)$ \\
& 10 & $0.0486(0.0022)$ & $0.0464(0.0021)$ \\
& 30 & $0.0520(0.0022)$ & $0.0509(0.0022)$ \\
& 50 & $0.0517(0.0022)$ & $0.0503(0.0022)$ \\
& 100 & $0.0512(0.0022)$ & $0.0489(0.0022)$ \\
\hline 0.7 & 7 & $0.0544(0.0023)$ & $0.0463(0.0021)$ \\
& 8 & $0.0519(0.0022)$ & $0.0495(0.0022)$ \\
& 9 & $0.0516(0.0022)$ & $0.0519(0.0022)$ \\
& 10 & $0.0484(0.0021)$ & $0.0518(0.0022)$ \\
& 30 & $0.0511(0.0022)$ & $0.0483(0.0021)$ \\
& 50 & $0.0530(0.0022)$ & $0.0511(0.0022)$ \\
& 100 & $0.0495(0.0022)$ & $0.0469(0.0021)$ \\
\hline 0.5 & 7 & $0.0542(0.0023)$ & $0.0498(0.0022)$ \\
& 8 & $0.0528(0.0022)$ & $0.0508(0.0022)$ \\
& 9 & $0.0511(0.0022)$ & $0.0530(0.0022)$ \\
& 10 & $0.0496(0.0022)$ & $0.0466(0.0021)$ \\
& 30 & $0.0503(0.0022)$ & $0.0498(0.0022)$ \\
& 50 & $0.0540(0.0023)$ & $0.0498(0.0022)$ \\
& 100 & $0.0499(0.0022)$ & $0.0463(0.0021)$ \\
\hline 0 & 7 & $0.0528(0.0022)$ & $0.0514(0.0022)$ \\
& 8 & $0.0503(0.0022)$ & $0.0510(0.0022)$ \\
& 9 & $0.0514(0.0022)$ & $0.0475(0.0021)$ \\
& 10 & $0.0484(0.0021)$ & $0.0463(0.0021)$ \\
& 30 & $0.0506(0.0022)$ & $0.0518(0.0022)$ \\
& 50 & $0.0540(0.0023)$ & $0.0498(0.0022)$ \\
100 & $0.0462(0.0021)$ & $0.0479(0.0021)$ \\
\hline & & &
\end{tabular}

Table 4.2: Estimated levels of a nominal level $\alpha=0.05$ test for Student's $t$-distribution $\left(T_{n}\right)$ with $n-2$ d.f. and permutation test based on $\hat{\Delta}_{1}$. The simulation is based on 10,000 replicates. The underlying distribution of $\epsilon$ is $N(0,1), \sigma_{1}=1$, and $\sigma_{2}=4$. Standard errors are given in parentheses. 


\begin{tabular}{cccc}
\hline$\sigma$ & $n$ & $T_{n}\left(\hat{\Delta}_{1}\right)$ & $\operatorname{Perm}\left(\hat{\Delta}_{1}\right)$ \\
\hline 5 & 7 & $0.0648(0.0025)$ & $0.0487(0.0022)$ \\
& 8 & $0.0533(0.0022)$ & $0.0500(0.0022)$ \\
& 9 & $0.0480(0.0021)$ & $0.0465(0.0021)$ \\
& 10 & $0.0460(0.0021)$ & $0.0503(0.0022)$ \\
& 30 & $0.0497(0.0022)$ & $0.0535(0.0023)$ \\
& 50 & $0.0527(0.0022)$ & $0.0504(0.0022)$ \\
& 100 & $0.0457(0.0021)$ & $0.0490(0.0022)$ \\
\hline 10 & 7 & $0.0672(0.0025)$ & $0.0546(0.0023)$ \\
& 8 & $0.0536(0.0023)$ & $0.0502(0.0022)$ \\
& 9 & $0.0525(0.0022)$ & $0.0472(0.0021)$ \\
& 10 & $0.0470(0.0021)$ & $0.0468(0.0021)$ \\
& 30 & $0.0510(0.0022)$ & $0.0530(0.0022)$ \\
& 50 & $0.0537(0.0023)$ & $0.0497(0.0022)$ \\
& 100 & $0.0500(0.0022)$ & $0.0487(0.0022)$ \\
\hline 20 & 7 & $0.0629(0.0024)$ & $0.0490(0.0022)$ \\
& 8 & $0.0490(0.0022)$ & $0.0505(0.0022)$ \\
& 9 & $0.0513(0.0022)$ & $0.0519(0.0022)$ \\
& 10 & $0.0461(0.0021)$ & $0.0502(0.0022)$ \\
& 30 & $0.0539(0.0023)$ & $0.0507(0.0022)$ \\
& 50 & $0.0513(0.0022)$ & $0.0489(0.0022)$ \\
& 100 & $0.0479(0.0021)$ & $0.0516(0.0022)$ \\
\hline 40 & 7 & $0.0599(0.0024)$ & $0.0465(0.0021)$ \\
& 8 & $0.0536(0.0023)$ & $0.0507(0.0022)$ \\
& 9 & $0.0516(0.0022)$ & $0.0517(0.0022)$ \\
& 10 & $0.0484(0.0021)$ & $0.0519(0.0022)$ \\
& 30 & $0.0516(0.0022)$ & $0.0512(0.0022)$ \\
& 50 & $0.0489(0.0022)$ & $0.0520(0.0022)$ \\
100 & $0.0480(0.0021)$ & $0.0473(0.0021)$ \\
\hline & & &
\end{tabular}

Table 4.3: Estimated levels of a nominal level $\alpha=0.05$ test for Student's $t$-distribution $\left(T_{n}\right)$ with $n-2$ d.f. and permutation test based on $\hat{\Delta}_{1}$. The simulation is based on 10,000 replicates, $\sigma_{1}=\sigma_{2}$. The underlying distribution for $\epsilon$ is $t$-distribution with d.f. being $5,10,20$, and 40. Standard errors are given in parentheses. 


\begin{tabular}{cccc}
\hline$\sigma$ & $n$ & $T_{n}\left(\hat{\Delta}_{1}\right)$ & $\operatorname{Perm}\left(\hat{\Delta}_{1}\right)$ \\
\hline 0.5 & 7 & $0.0605(0.0024)$ & $0.0488(0.0022)$ \\
& 8 & $0.0541(0.0023)$ & $0.0487(0.0022)$ \\
& 9 & $0.0435(0.0020)$ & $0.0455(0.0021)$ \\
& 10 & $0.0428(0.0020)$ & $0.0485(0.0021)$ \\
& 30 & $0.0447(0.0021)$ & $0.0515(0.0022)$ \\
& 50 & $0.0501(0.0022)$ & $0.0498(0.0022)$ \\
& 100 & $0.0541(0.0023)$ & $0.0518(0.0022)$ \\
\hline 1 & 7 & $0.0513(0.0022)$ & $0.0515(0.0022)$ \\
& 8 & $0.0477(0.0021)$ & $0.0434(0.0020)$ \\
& 9 & $0.0380(0.0019)$ & $0.0541(0.0023)$ \\
& 10 & $0.0398(0.0020)$ & $0.0515(0.0022)$ \\
& 30 & $0.0431(0.0020)$ & $0.0519(0.0022)$ \\
& 50 & $0.0486(0.0022)$ & $0.0501(0.0022)$ \\
& 100 & $0.0483(0.0021)$ & $0.0516(0.0022)$ \\
\hline 2 & 7 & $0.0370(0.0019)$ & $0.0477(0.0021)$ \\
& 8 & $0.0321(0.0018)$ & $0.0506(0.0022)$ \\
& 9 & $0.0299(0.0017)$ & $0.0463(0.0021)$ \\
& 10 & $0.0322(0.0018)$ & $0.0503(0.0022)$ \\
& 30 & $0.0322(0.0018)$ & $0.0485(0.0021)$ \\
& 50 & $0.0371(0.0019)$ & $0.0499(0.0022)$ \\
100 & $0.0362(0.0019)$ & $0.0538(0.0023)$ \\
\hline 4 & 7 & $0.0280(0.0016)$ & $0.0512(0.0022)$ \\
& 8 & $0.0251(0.0016)$ & $0.0501(0.0022)$ \\
& 9 & $0.0202(0.0014)$ & $0.0496(0.0022)$ \\
& 10 & $0.0221(0.0015)$ & $0.0505(0.0022)$ \\
30 & $0.0184(0.0013)$ & $0.0475(0.0021)$ \\
& 50 & $0.0199(0.0014)$ & $0.0475(0.0021)$ \\
100 & $0.0194(0.0014)$ & $0.0450(0.0021)$ \\
\hline & & &
\end{tabular}

Table 4.4: Estimated levels of a nominal level $\alpha=0.05$ test for Student's $t$-distribution $\left(T_{n}\right)$ with $n-2$ d.f. and permutation test based on $\hat{\Delta}_{1}$. The simulation is based on 10,000 replicates, $\sigma_{1}=\sigma_{2}$. The underlying distribution for $\epsilon$ is lognormal with mean of $\log (\epsilon)$ to be 0 and standard deviation of $\log (\epsilon)$ to be $0.5,1,2,4$ respectively. Standard errors are given in parentheses. 
perfect ranking with $n=7$

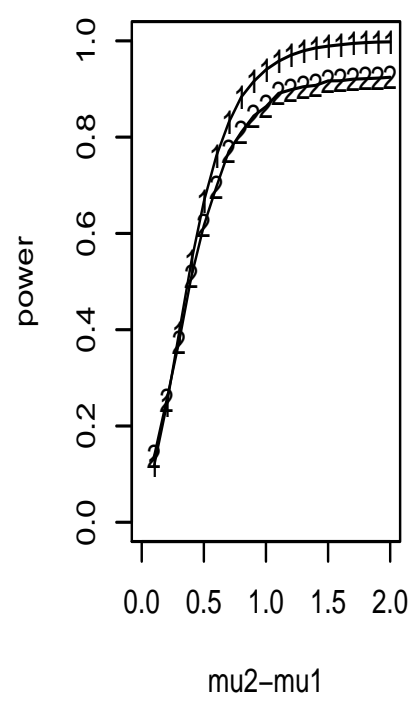

perfect ranking with $n=50$

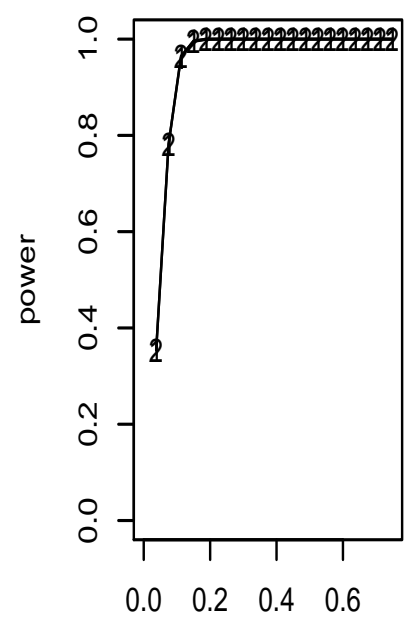

(mu2-mu1)*sqrt(7/50) perfect ranking with $\mathrm{n}=10$
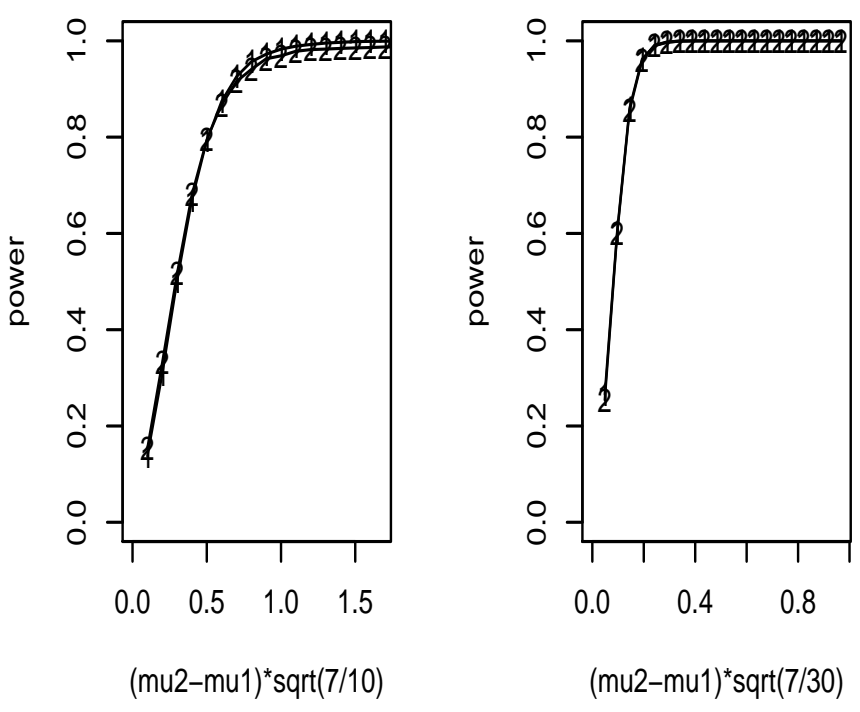

perfect ranking with $\mathrm{n}=30$

$($ mu2-mu1)*sqrt(7/30)

perfect ranking with $n=10($

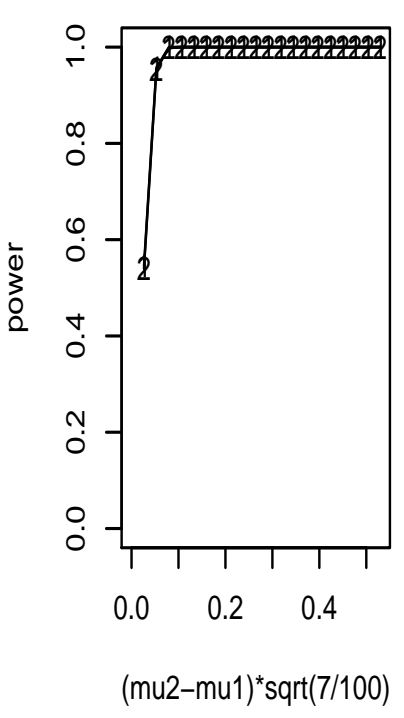

Figure 4.5: Empirical power of $T_{n}$ with $n-2$ d.f. with label 1 and permutation test based on $\hat{\Delta}_{1}$ with label 2 for perfect ranking. The simulation is based on 10,000 replicates. The underlying distribution for $\epsilon$ is lognormal with mean of $\log (\epsilon)$ to be 0 and standard deviation of $\log (\epsilon)$ to be $0.5, \sigma_{1}=\sigma_{2}=1$. 
perfect ranking with $n=7$

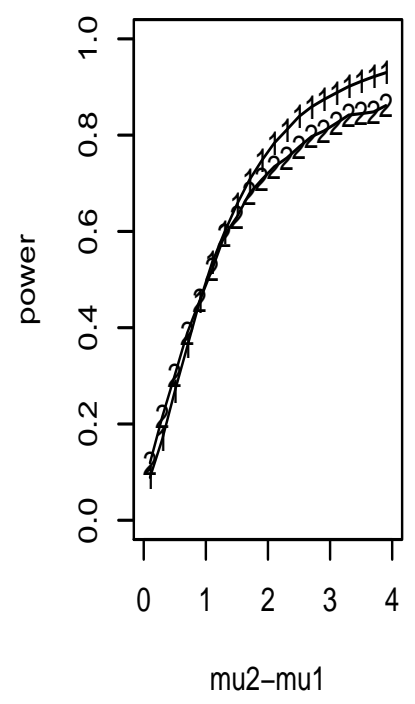

perfect ranking with $\mathrm{n}=50$

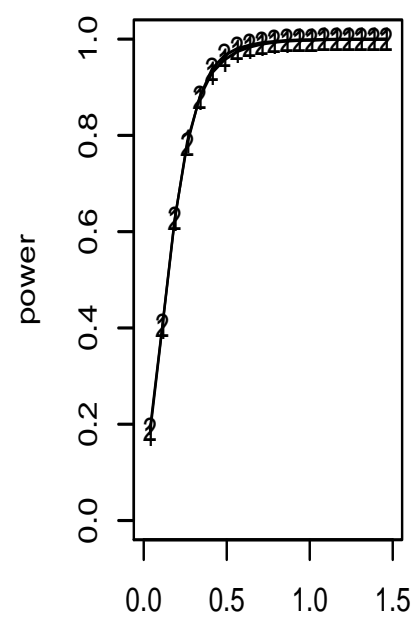

(mu2-mu1)*sqrt(7/50) perfect ranking with $\mathrm{n}=10$
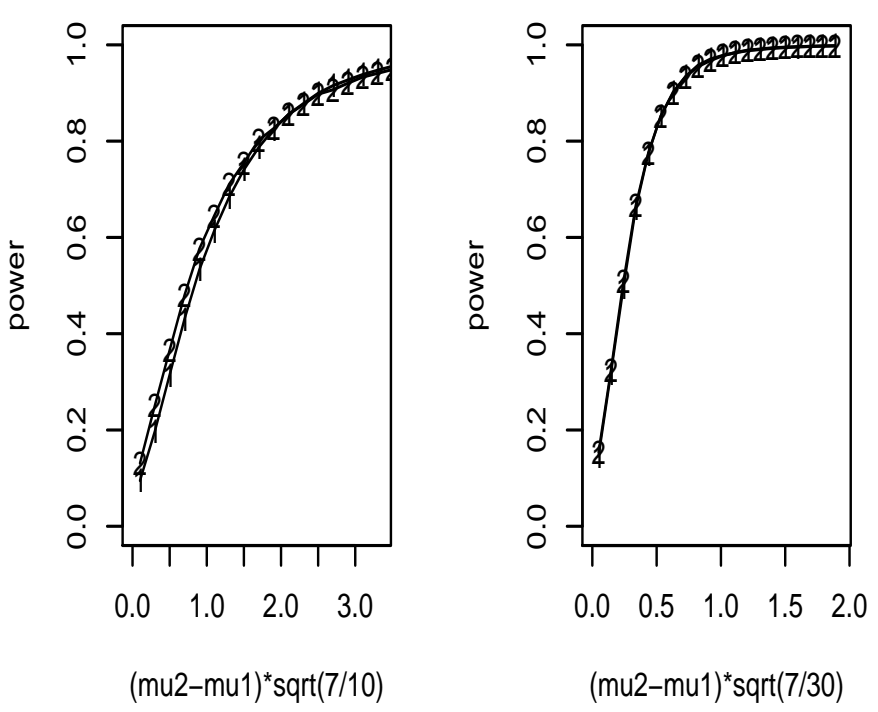

perfect ranking with $\mathrm{n}=30$

perfect ranking with $n=10 c$

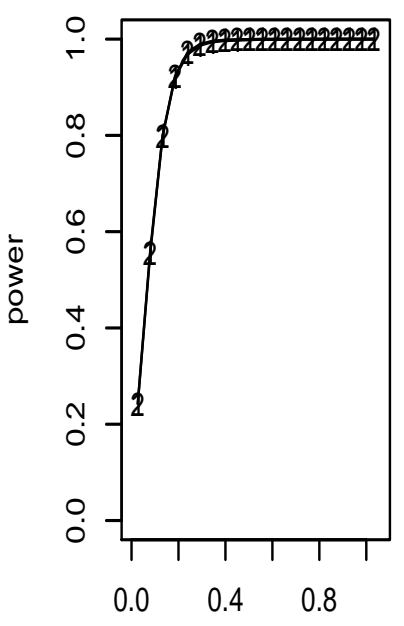

(mu2-mu1)*sqrt(7/100)

Figure 4.6: Empirical power of $T_{n}$ with $n-2$ d.f. with label 1 and permutation test based on $\hat{\Delta}_{1}$ with label 2 for perfect ranking. The simulation is based on 10,000 replicates. The underlying distribution for $\epsilon$ is $\log$ normal with mean of $\log (\epsilon)$ to be 0 and standard deviation of $\log (\epsilon)$ to be $1, \sigma_{1}=\sigma_{2}=1$. 
perfect ranking with $n=7$

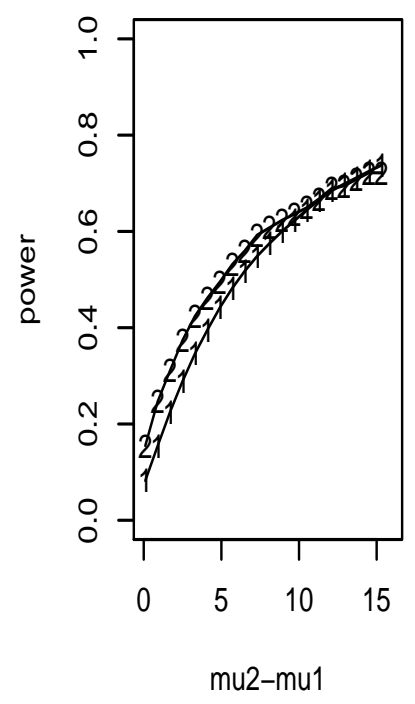

perfect ranking with $\mathrm{n}=50$

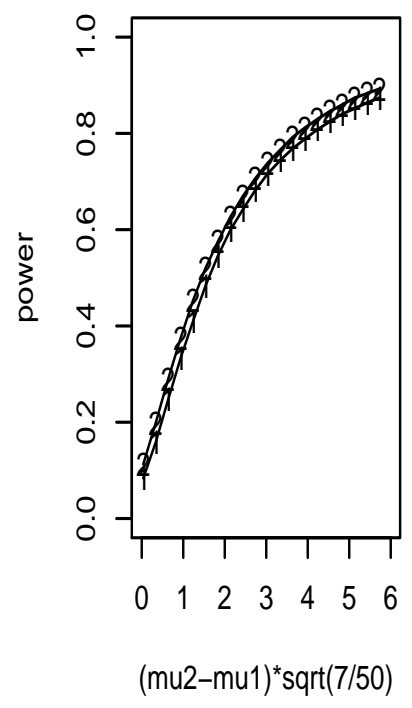

perfect ranking with $n=10$

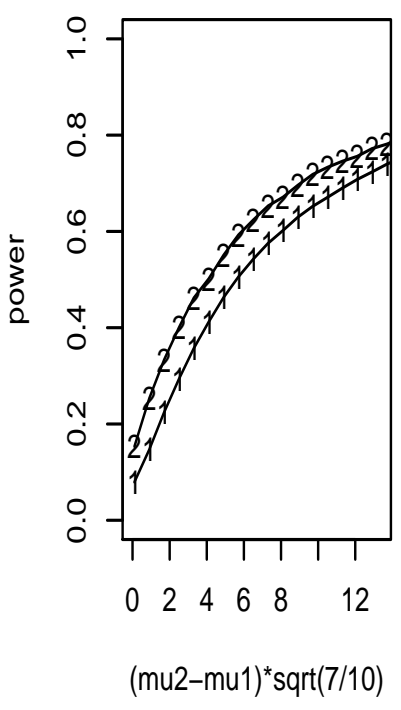

perfect ranking with $\mathrm{n}=10 \mathrm{c}$

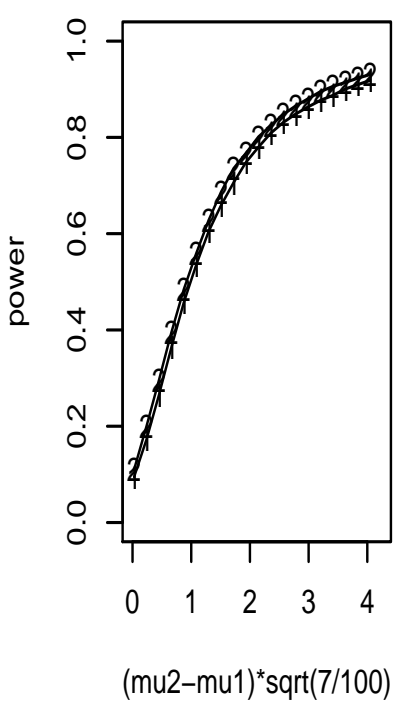

perfect ranking with $\mathrm{n}=30$

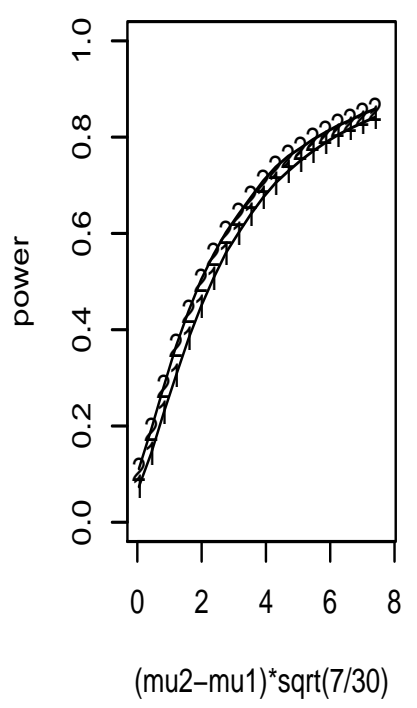

Figure 4.7: Empirical power of $T_{n}$ with $n-2$ d.f. with label 1 and permutation test based on $\hat{\Delta}_{1}$ with label 2 for perfect ranking. The simulation is based on 10,000 replicates. The underlying distribution for $\epsilon$ is lognormal with mean of $\log (\epsilon)$ to be 0 and standard deviation of $\log (\epsilon)$ to be $2, \sigma_{1}=\sigma_{2}=1$. 
perfect ranking with $n=7$

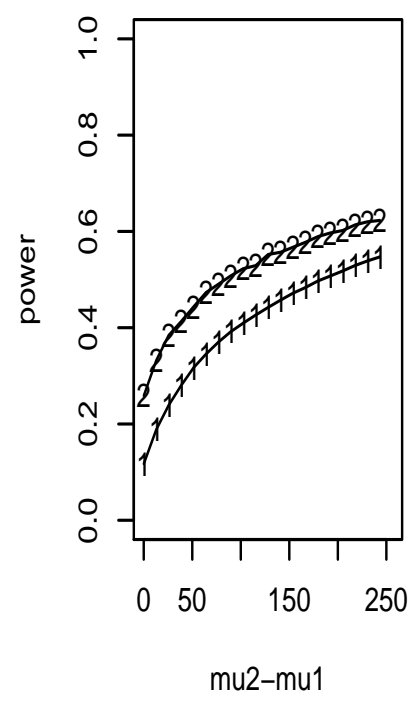

perfect ranking with $n=50$

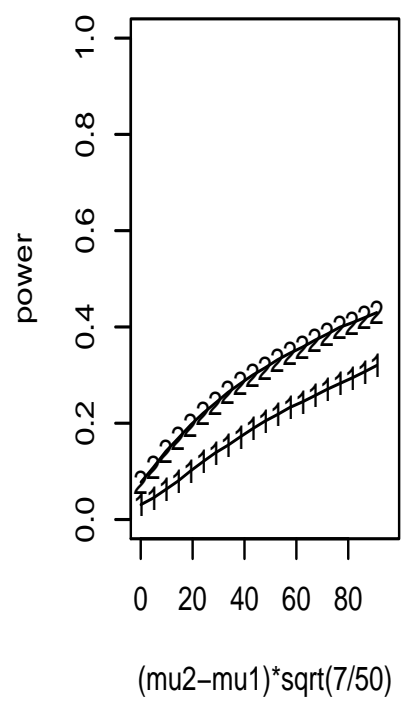

perfect ranking with $n=10$

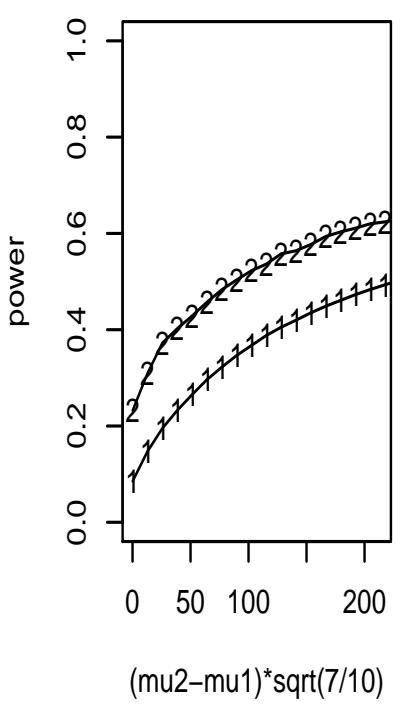

perfect ranking with $\mathrm{n}=10 \mathrm{c}$

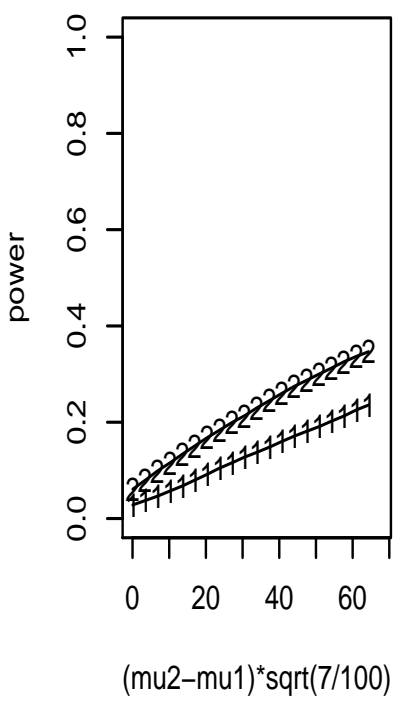

perfect ranking with $\mathrm{n}=30$

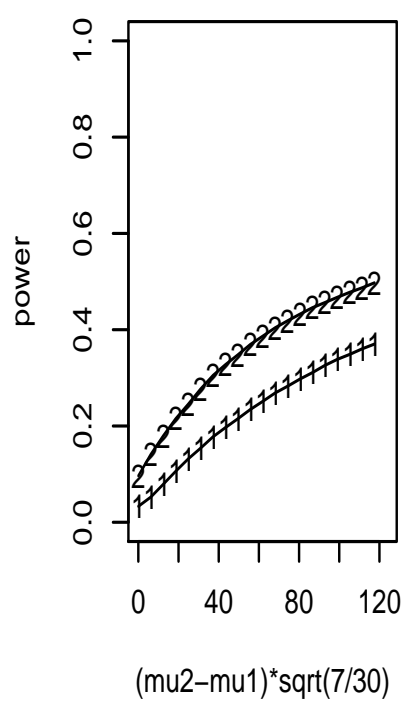

Figure 4.8: Empirical power of $T_{n}$ with $n-2$ d.f. with label 1 and permutation test based on $\hat{\Delta}_{1}$ with label 2 for perfect ranking. The simulation is based on 10,000 replicates. The underlying distribution for $\epsilon$ is lognormal with mean of $\log (\epsilon)$ to be 0 and standard deviation of $\log (\epsilon)$ to be $4, \sigma_{1}=\sigma_{2}=1$. 


\section{CHAPTER 5}

\section{LATEX BALLOON MANUFACTURE}

Balloons are manufactured in many different colors. To make a balloon, pigment is added to liquid latex. The strength and other characteristics of the balloon can be affected by the characteristics of the pigment. A primary concern is the size of the particles in the pigment. An experimenter observed that some colors of birthday balloons seem to be harder to inflate than others. She ran an experiment to determine whether balloons of different colors are similar in terms of the time taken for inflation to a diameter of 7 inches. Balloons of four colors were selected from one manufacturer. With the help of an assistant, the experimenter recorded the inflation times (to the nearest $1 / 10$ second). The run order for each experimental unit is also recorded. Dean and Voss (1999) present data which are reproduced in Table 5.1.

Dean and Voss (1999, p.285) note that the residuals from a one-way analysis of variance show a relationship with run order. They include run order as a linear covariate and perform an analysis of covariance (ANCOVA). Results, including estimation for each pairwise comparison of treatment effects, are presented in Table 9.6 of Dean and Voss (1999). The residuals from the ANCOVA are plotted against run order in Figure 5.1. At first glance, the residuals appear acceptable, although there may be some evidence of a bowl-shaped pattern. When broken out by color, however, 
the residuals show patterns. For example, the residuals for yellow (color 2) show a decreasing pattern. Thus, further improvement in the analysis is possible.

We analyze each of the six pairs of colors, treating each pair as a separate twosample problem. This allows us to compare the new estimators to $\hat{\Delta}$ and $\hat{\Delta}_{A N C O V A}$ across a range of conditions. For each pair of treatments, sets are randomly formed and ranks are created for all sets by using run order. The estimators $\hat{\Delta}_{1}, \hat{\Delta}_{3}, \hat{\Delta}$, and $\hat{\Delta}_{A N C O V A}$ are computed. Assuming equal variances for each pair of treatments, pooled $t$ procedures are used to determine the reference distribution to obtain $\mathrm{p}$ values. Results are listed in Table 5.2.

For this data set, the VRBE and ANCOVA models deliver better performance than does the ANOVA model. None of the methods find any difference in the pink-blue or yellow-orange comparisons. Differences in the other four comparisons seem to be real and of substantial size. The VRBE and ANCOVA result in larger test statistics and smaller p-values for the other color comparisons as well. The point estimates vary among the methods, but the standard errors are consistently smaller for those that adjust for run order. The standard errors for $\hat{\Delta}_{3}$ and $\hat{\Delta}_{A N C O V A}$ are comparable. To our eyes, $\hat{\Delta}_{1}$ appears to provide better performance than $\hat{\Delta}$, in keeping with the earlier theoretical development and simulation studies.

We report the permutation test results in Table 5.3. The p-values are relatively large in this case because of the discrete nature of the test statistic. According to the permutation procedure, conditional on $n_{1}$, we only have $\left(\begin{array}{c}n \\ n_{1}\end{array}\right)$ possible permutations. Therefore, following the two-sided test we derived in previous chapter, the smallest p-value we can achieve is $\frac{2}{\left(\begin{array}{c}n \\ n_{1}\end{array}\right)}$. Let us take the comparison between color 1 and color 3 as an example. Since $n_{1}=5$, we then have $\left(\begin{array}{c}n \\ n_{1}\end{array}\right)=56$ possible $\hat{\Delta}_{1}^{*}$ s. In this case, 


\begin{tabular}{cccccc}
\hline \hline Color & Order & Time & Color & Order & Time \\
\hline 1 & 1 & 22.43 & 2 & 6 & 22.20 \\
1 & 3 & 20.33 & 2 & 7 & 28.50 \\
1 & 10 & 19.60 & 2 & 8 & 25.70 \\
1 & 16 & 15.80 & 2 & 11 & 28.80 \\
1 & 18 & 17.50 & 2 & 17 & 18.30 \\
1 & 21 & 16.30 & 2 & 24 & 18.10 \\
1 & 30 & 19.30 & 2 & 25 & 18.90 \\
1 & 31 & 15.90 & 2 & 27 & 20.10 \\
\hline 3 & 2 & 24.60 & 4 & 4 & 19.80 \\
3 & 5 & 24.30 & 4 & 12 & 24.00 \\
3 & 9 & 20.20 & 4 & 13 & 17.10 \\
3 & 15 & 24.20 & 4 & 14 & 19.30 \\
3 & 20 & 22.90 & 4 & 19 & 18.70 \\
3 & 28 & 22.50 & 4 & 22 & 14.00 \\
3 & 29 & 16.00 & 4 & 23 & 16.60 \\
3 & 32 & 20.30 & 4 & 26 & 16.00 \\
\hline \hline
\end{tabular}

Table 5.1: Times (in seconds) for the balloon experiment, where the codes 1, 2, 3, 4 denote the color pink, yellow, orange and blue.

although our estimator $\hat{\Delta}_{1}$ turns out to be the smallest value among these 56 possible values, our p-value for two sided test is $\frac{2}{56}=0.0357$. Therefore, the p-value itself cannot give us the complete picture. We need to take discreteness of the data into consideration. As we showed in Chapter 4, we could use a randomized version of the permutation test to obtain level $\alpha$. 


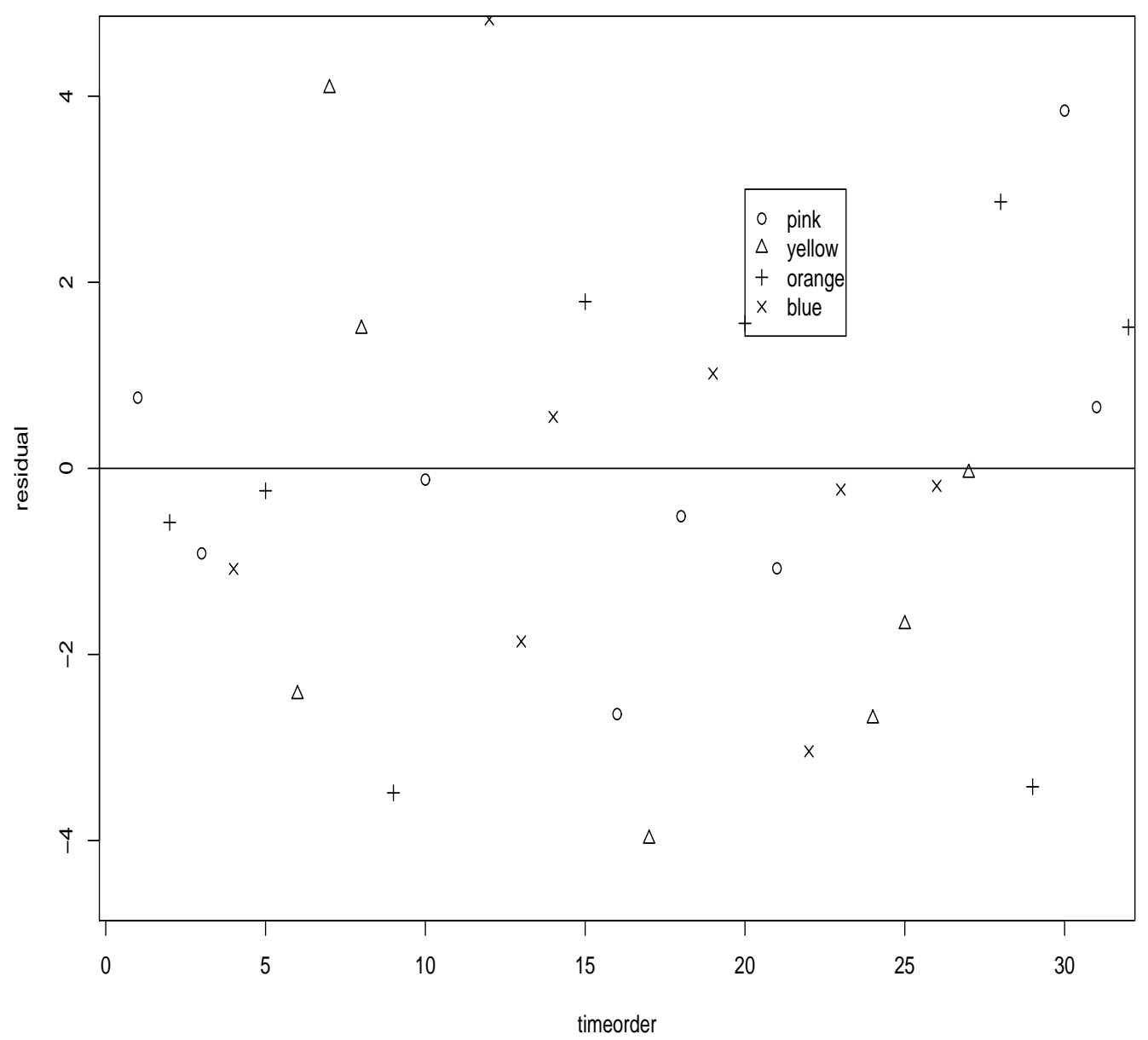

Figure 5.1: Residual plot for analysis of covariance model. 


\begin{tabular}{ccccccc}
\hline Contrast & Method & D.F. & Estimate & S.E. & T(Z)Value & P-Value \\
\hline $1-2$ & $\hat{\Delta}$ & 14 & -4.1875 & 1.8017 & -2.3242 & 0.0357 \\
& $\hat{\Delta}_{\text {ANCOVA }}$ & 13 & -4.0724 & 1.4538 & -2.8010 & 0.0150 \\
& $\hat{\Delta}_{1}$ & 6 & -3.1033 & 1.4416 & -2.1527 & 0.0748 \\
& $\hat{\Delta}_{3}$ & 7 & -4.1103 & 1.4459 & -2.8426 & 0.0250 \\
\hline $1-3$ & $\hat{\Delta}$ & 14 & -3.4875 & 1.3366 & -2.6092 & 0.0206 \\
& $\hat{\Delta}_{\text {ANCOVA }}$ & 13 & -3.5758 & 1.0538 & -3.3930 & 0.0048 \\
& $\hat{\Delta}_{1}$ & 6 & -3.9367 & 1.2044 & -3.2685 & 0.0170 \\
& $\hat{\Delta}_{3}$ & 7 & -3.5438 & 1.0841 & -3.2689 & 0.0136 \\
\hline $1-4$ & $\hat{\Delta}$ & 14 & 0.2000 & 1.3628 & 0.1468 & 0.8854 \\
& $\hat{\Delta}_{\text {ANCOVA }}$ & 13 & 0.0137 & 1.0479 & 0.0130 & 0.9900 \\
& $\hat{\Delta}_{1}$ & 6 & 0.2000 & 1.3605 & 0.1470 & 0.8879 \\
& $\hat{\Delta}_{3}$ & 7 & 0.0820 & 1.1161 & 0.0734 & 0.9435 \\
\hline $2-3$ & $\hat{\Delta}$ & 14 & 0.7000 & 1.8976 & 0.3689 & 0.7177 \\
& $\hat{\Delta}_{A N C O V A}$ & 13 & 0.2226 & 1.5601 & 0.1430 & 0.8887 \\
& $\hat{\Delta}_{1}$ & 6 & 0.7000 & 1.6921 & 0.4137 & 0.6935 \\
& $\hat{\Delta}_{3}$ & 7 & 0.3849 & 1.5547 & 0.2475 & 0.8115 \\
\hline $2-4$ & $\hat{\Delta}$ & 14 & 4.3875 & 1.9161 & 2.2898 & 0.0381 \\
& $\hat{\Delta}_{\text {ANCOVA }}$ & 13 & 4.1190 & 1.4420 & 2.8560 & 0.0135 \\
& $\hat{\Delta}_{1}$ & 6 & 5.3167 & 1.6202 & 3.2815 & 0.0168 \\
& $\hat{\Delta}_{3}$ & 7 & 4.1967 & 1.4121 & 2.9718 & 0.0207 \\
\hline $3-4$ & $\hat{\Delta}$ & 14 & 3.6875 & 1.4872 & 2.4794 & 0.0265 \\
& $\hat{\Delta}_{\text {ANCOVA }}$ & 13 & 3.9713 & 1.2089 & 3.2850 & 0.0059 \\
& $\hat{\Delta}_{1}$ & 6 & 4.9250 & 1.1966 & 4.1155 & 0.0062 \\
& $\hat{\Delta}_{3}$ & 7 & 3.8591 & 1.2691 & 3.0408 & 0.0188 \\
\hline & & & & & &
\end{tabular}

Table 5.2: Pairwise comparisons for four methods including the SRS estimator $\hat{\Delta}$, ANCOVA $\hat{\Delta}_{A N C O V A}$, JPS estimator $\hat{\Delta}_{1}$ and VRBE $\hat{\Delta}_{3}$, where the codes $1,2,3,4$ denote the colors pink, yellow, orange and blue, respectively. 


\begin{tabular}{ccccccc}
\hline Contrast & $1-2$ & $1-3$ & $1-4$ & $2-3$ & $2-4$ & $3-4$ \\
\hline$\hat{\Delta}_{1}$ & -3.1033 & -3.9367 & 0.200 & 0.700 & 5.3167 & 4.925 \\
$n_{1}$ & 3 & 5 & 4 & 4 & 3 & 2 \\
p-value & 0.0714 & 0.0357 & 0.8571 & 0.7714 & 0.0714 & 0.0714 \\
\hline
\end{tabular}

Table 5.3: Pairwise comparisons of the inflation times for the four colors using the permutation test. 


\section{CHAPTER 6}

\section{DISCUSSION}

\subsection{Conclusions}

The two-sample, completely randomized design is perhaps the most commonly used experimental design. The standard estimator of the treatment contrast ignores any additional information about the experimental units. Traditional techniques such as ANCOVA apply only when the information can be translated into a small number of numerical covariates. The methodology that we developed in this work provides a means of exploiting this additional (perhaps subjective or difficult to quantify) information in a fully objective fashion.

The methodology we develop does not require any specific form connecting the explanatory information to the responses - hence we do not need to make any assumptions such as a linear effect of some created covariate. From one viewpoint, the analysis we propose provides a nonparametric analysis of covariance. We believe that this approach is more in keeping with the qualitative information about experimental units than is traditional ANCOVA.

The methods provided in this work require one to rank units in each set of experimental units using the additional information. After all units within sets get 
ranked, the sets are divided into two types according to whether the rank 1 unit is in the treatment or control group. The JPS estimator $\hat{\Delta}_{1}$ is obtained by averaging the sample means for each type. We have shown that $\hat{\Delta}_{1}$ is an unbiased estimator of $\Delta$ and is as good as the SRS estimator $\hat{\Delta}$ when the ranking is performed randomly. As the ranking quality increases, $\hat{\Delta}_{1}$ gets more efficient than $\hat{\Delta}$. To further improve the estimator, we adapt the Rao-Blackwell Theorem and derive the RBE $\hat{\Delta}_{2}$ and the $\operatorname{VRBE} \hat{\Delta}_{3}$. Specifically, these two estimators are obtained by taking the expectation of $\hat{\Delta}_{1}$ conditional on the ranks and responses. Both numerical and theoretical studies show that $\hat{\Delta}_{3}$ is the best performer, followed by $\hat{\Delta}_{2}$, and then $\hat{\Delta}_{1}$. On the other hand, $\hat{\Delta}_{1}$ requires the least ranking information for the EUs among the three proposed estimators.

Although our methods do not allow the ranker to express indifference about the units in a set, they can be extended to allow indifference as well as judgments that lie between indifference and a determined ranking. Development of such an extension would follow along the lines of MacEachern et al. (2004) and Stokes and Wang (2005).

In the first part of this work, we pursue one major route to statistical inference: we develop theoretical results for the asymptotic distribution of the contrast. For $\hat{\Delta}_{1}$, Theorem 2 shows its asymptotic normality and the performance of the $t$-test based on $T_{n}$ and the $z$-test based on $Z_{n}$ are studied. By converting $\hat{\Delta}_{3}$ to a four-dimensional U-statistic, we utilize properties of U-statistics to obtain the asymptotic normality of $\hat{\Delta}_{3}$. The asymptotic results enable us to provide a full range of inferential techniques for the new analysis, including point estimation, hypothesis tests, and confidence intervals. 
The statistical inference procedures based on asymptotic theories require large sample sizes when the random error is not normally distributed. In the second part of this work, we have also developed methods for a second major route to statistical inference. We have examined a variety of permutation-based methods for inference. These methods help to robustify inference to the form of the underlying populations. Theorem 5 states equivalence of the permutation tests based on $\hat{\Delta}_{1}, \sum_{i=1}^{n_{1}} X_{i}, V_{n}$ and $T_{n}$. The asymptotic properties of the permutation tests and the asymptotic equivalence with the $t$-test based on $T_{n}$ are given in Theorem 6 . Our simulation results confirm that the proposed permutation tests preserve test levels very well for various distributions of the random error.

One major problem with the permutation tests is the discreteness of the permutation test statistic. This drawback is demonstrated by the p-values calculated for the permutation test in Chapter 5. One possible solution is to improve the permutation test via approximate conditioning (Pierce and Peters, 1999). Moreover, further improvements to the computational algorithms for small and moderate sample sizes are desirable, especially for the permutation test of $\hat{\Delta}_{3}$. In particular, the algorithm proposed by Diaconis and Holmes (1994) may be considered.

\subsection{Future Directions}

Our current estimation procedures are developed for completely randomized twosample designs. One direct generalization is to apply the procedure to cases with multiple samples. Examples include the effects of new drugs with several doses. In that case, one wants to estimate the effects for more than two treatments. Utilizing extra information like health conditions of the patients can improve estimation 
accuracy. Although a multiple-treatment problem can be solved by a sequence of two-sample problems, a more general and simplified method is desirable.

Another extension is to consider more general models such as generalized linear models. Then responses can be binary variables, counts, and probabilities. Moreover, the procedure can be extended to scenarios where both treatments and other numerical covariates are already included in the model. This aims to incorporate covariates in the estimation procedure, which allows us to further improve inference based on the ANCOVA model. Finally, the proposed procedures may have many potential applications on real data sets, especially in clinical trials. 


\section{APPENDIX A}

\section{A.1 Comparing $\hat{\Delta}, \hat{\Delta}_{1}, \hat{\Delta}_{2}$, and $\hat{\Delta}_{3}$.}

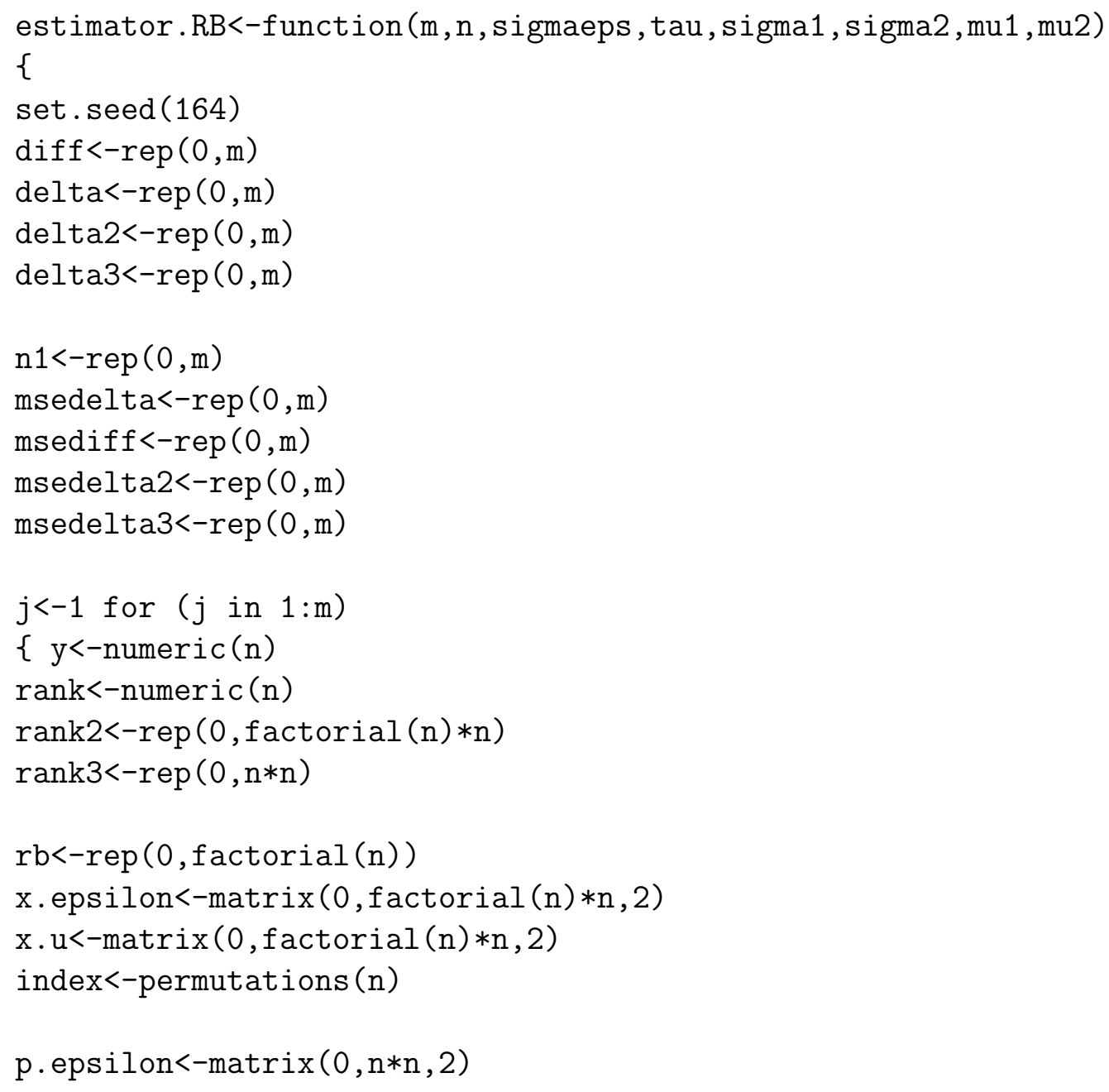




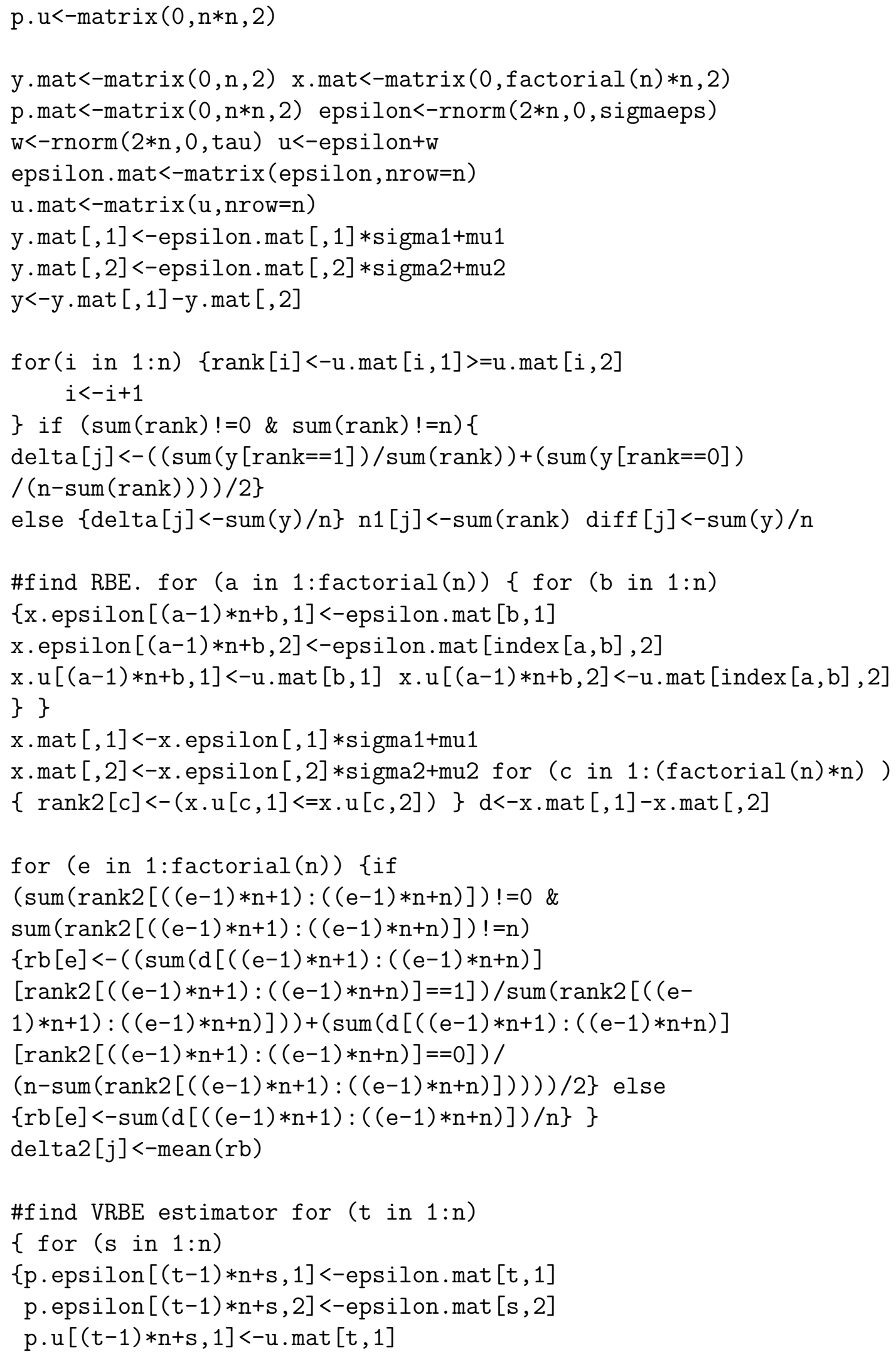




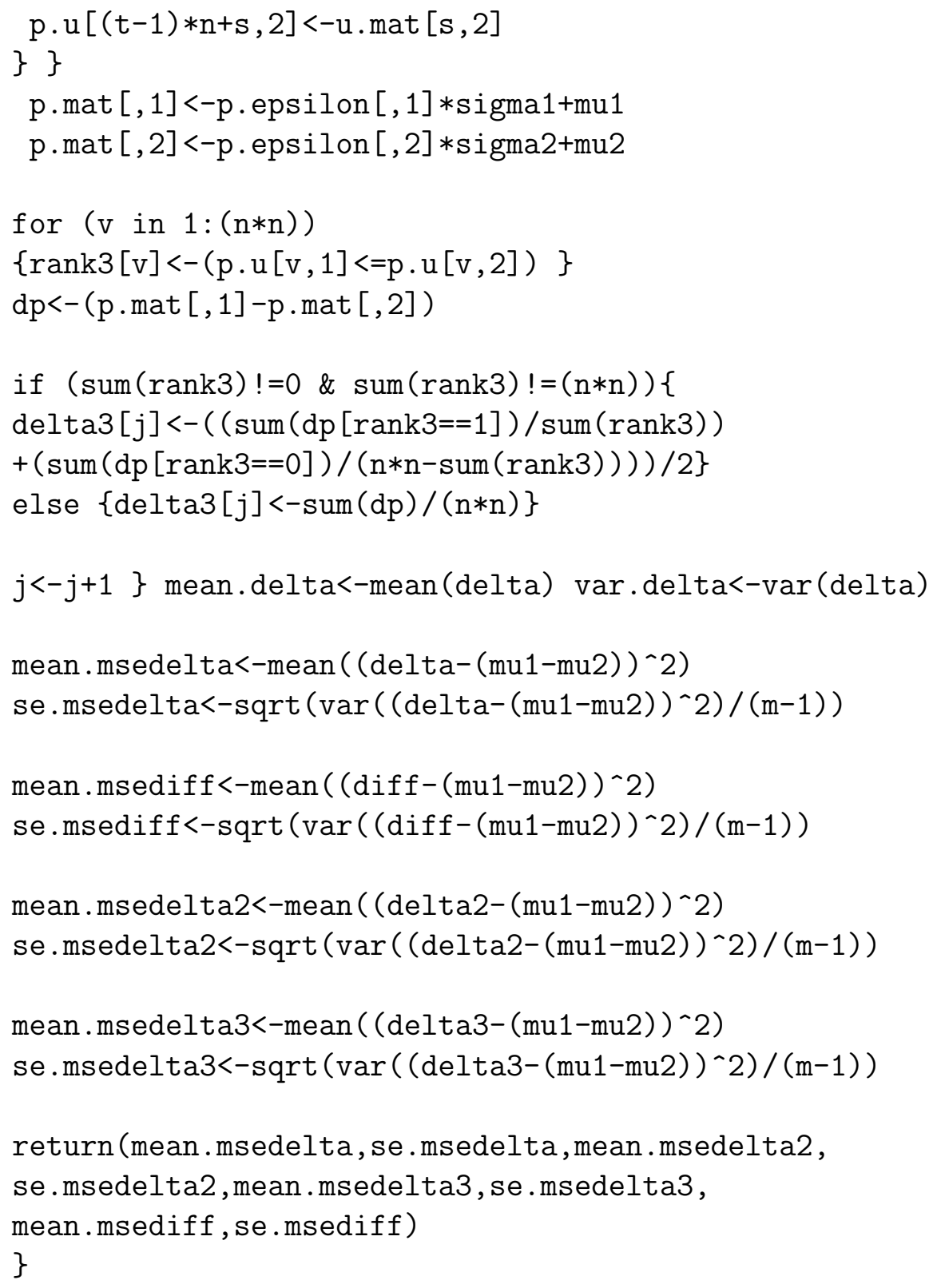

\section{A.2 Calculating Estimated Levels for Test Statistics based on Asymptotic Theory.}




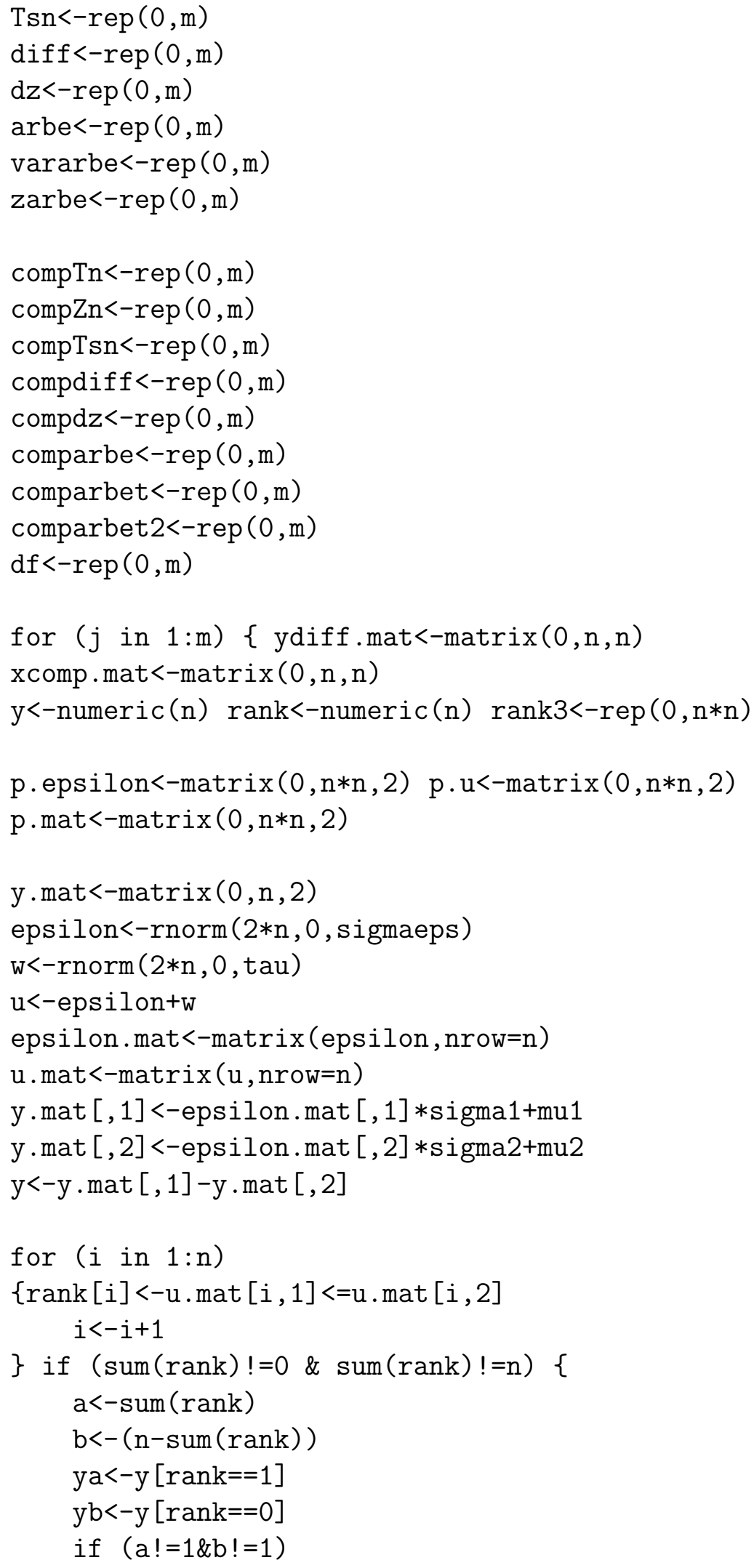




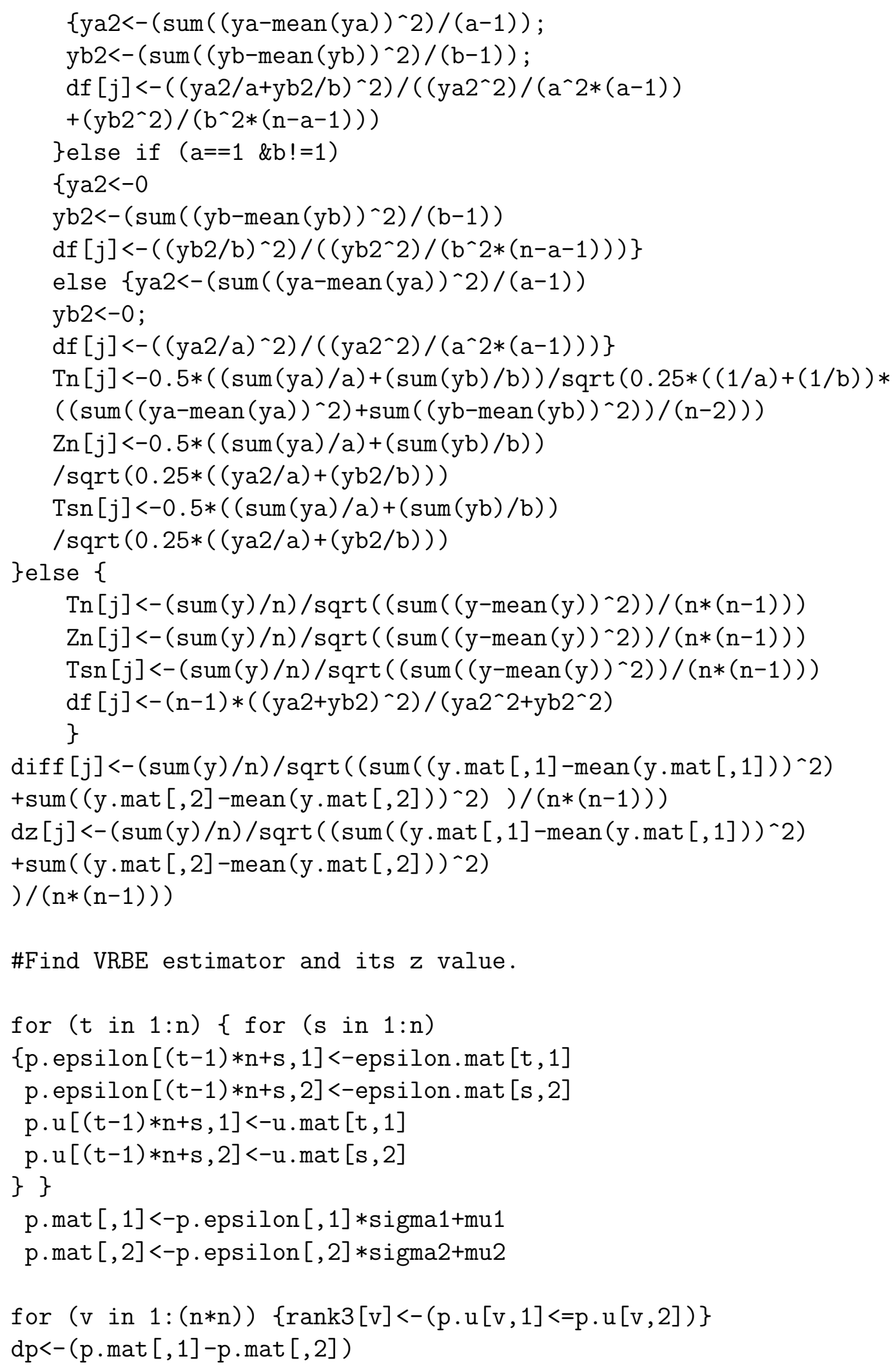




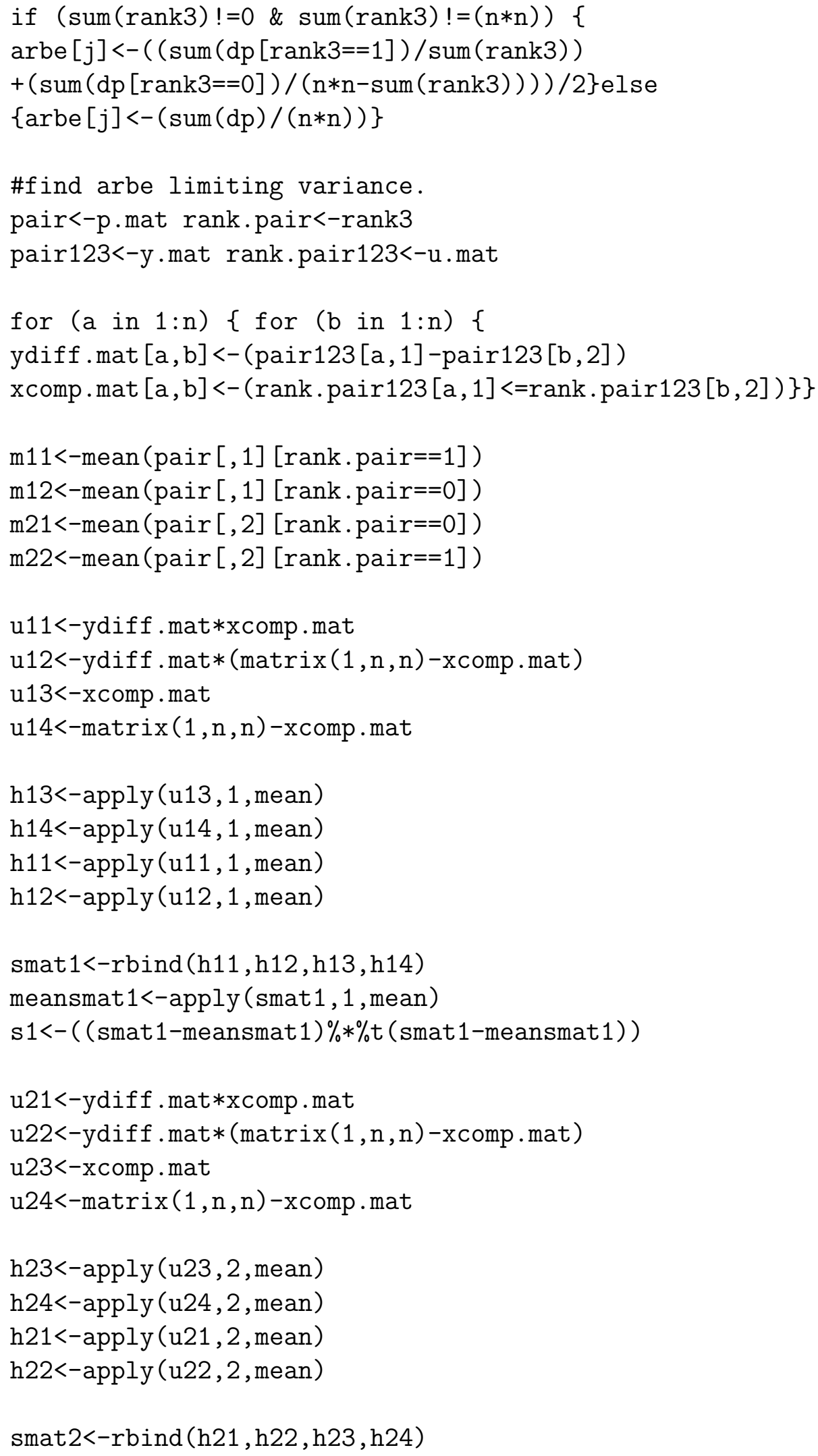




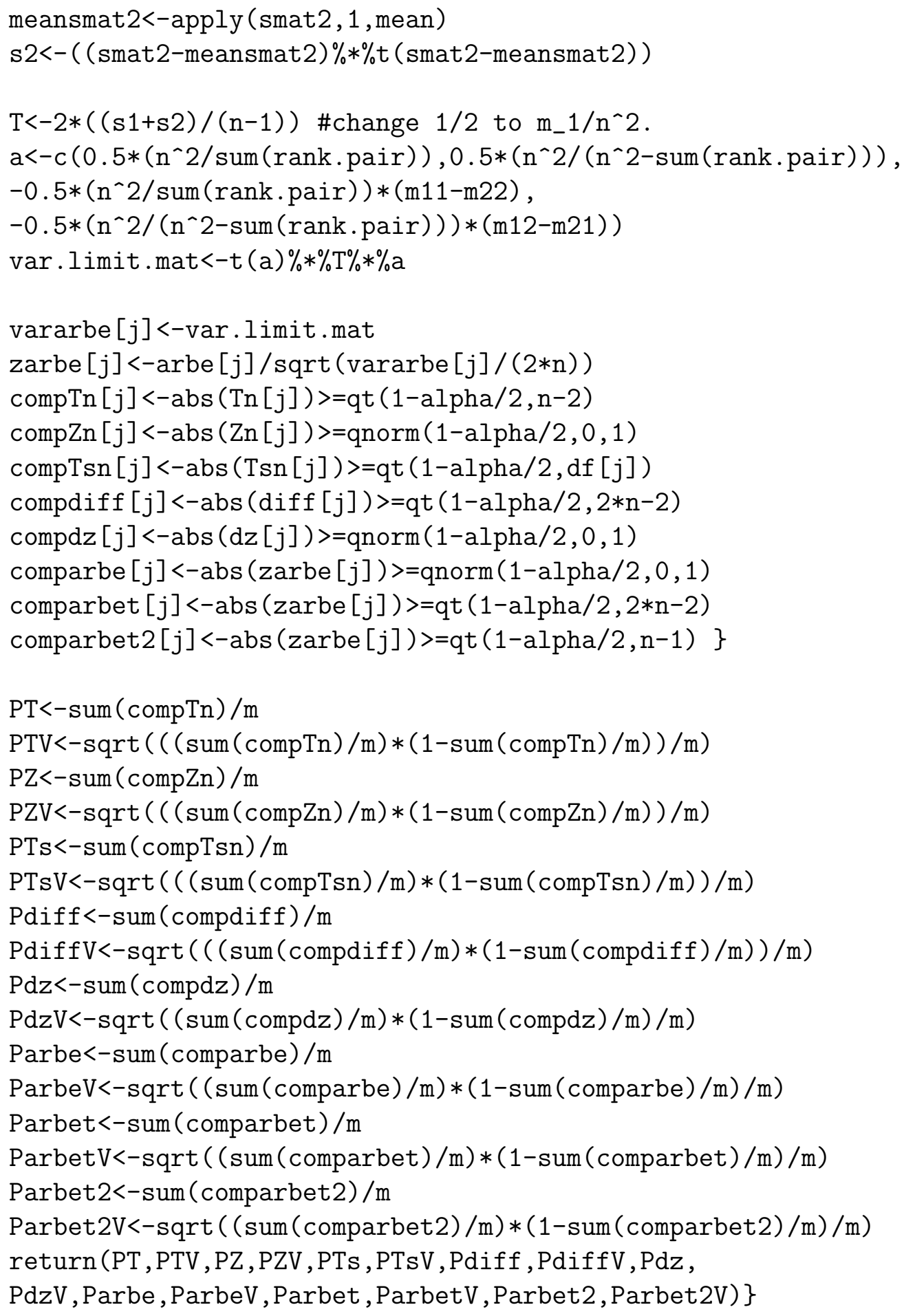




\section{BIBLIOGRAPHY}

[1] Barnett, V. (1999). Ranked set sample design for environmental investigation. Environ. Ecol. Stat.,6, 59-74.

[2] Bhoj, D. S. (1996). Estimation of parameters of generalized geometric distribution using ranked set sampling. Biometrics, 52, 685-694.

[3] Bhoj, D. S. (1997). Estimation of parameters of the extreme value distribution using ranked set sampling. Communications in Statistics- Theory and Methods, $26,653-$.

[4] Bohn, L. L. and Wolfe, D. A. (1992). Nonparametric two-sample procedures for ranked-set samples data. Journal of the American Statistical Association, 87, $552-561$.

[5] Bohn, L. L. and Wolfe, D. A. (1994). The effect of imperfect judgement rankings on properties of procedures based on the ranked-set samples analog of the MannWhitney-Wilcoxon statistics. Journal of the American Statistical Association, 87, $552-561$.

[6] Chen, H., Stasny, E. A., and Wolfe, D. A. (2005). Unbalanced ranked set sampling for estimating a population proportion. To appear in Biometrics.

[7] Chen, Z. (2000). On ranked set sample quantiles and their applications. Journal of statistical Planning and Inference, 83, 125-135.

[8] Chen, Z. (2001). The optimal ranked set sampling scheme for inference on population quantiles. Statistica Sinica,11, 23-27.

[9] Chen, Z., Bai, Z., and Sinha, B. K. (2003). Ranked set samping: Theory and Applications. Springer.

[10] David, H. A. and Levine, D. N. (1972). Ranked set sampling in the presence of judgement error. Biometrics, 28, 553-555.

[11] Dean, A. and Voss, D. (1999). Design and Analysis of Experiments. SringerVerlag. 
[12] Dell, T. R. and Clutter, J. L. (1972). Ranked set sampling theory with order statistics background. Biometrics, 28, 545-555.

[13] Diaconis, P. and Holmes, S. (1994). Gray codes for randomization procedures. Statistics and Computing, 4:287-302.

[14] Evans, M. J. (1967). Applications of ranked set sampling to regeneration surveys in areas direct seeded to long-leaf pine. Master of Forestry Dissertation, Louisiana State University, Baton Rouge, LA.

[15] Fei, H., Sinha, B. K., and Wu, Z. (1994). Estimation of parameters in twoparameter Weibull and extreme-value distribution using ranked set sampling. Journal of statistical Research, 28, 149-161.

[16] Fligner, M. A. and MacEchern, S. N. (2004). Nonparametric two-sample methods for ranked set sample data. Tentatively Accepted for JASA.

[17] Good, P. (1994). Permutation Tests. Springer Verlag, New York, NY.

[18] Halls, L. S. and Dell, T. R. (1966). Trial of ranked set sampling for forage yields. Forest Science, 12(1), 22-26.

[19] Husby, C.E., Stasny, E. A., and Wolfe, D. A. (2005). An application of ranked set sampling for mean and median estimation using USDA crop production data, Journal of Agricultural, Biological, and Environmental Statistics. 10(3), 1-20.

[20] Kaur, A., Patil, G. P., Sinha, A. K., and Taillie, C. (1995). Ranked set sampling: An annotated bibliography. Environmental and Ecological Statistics, 2, 25-54.

[21] Kaur, A. Patil, G. P. and taillie, C. (1997). Unequal allocation models for ranked set sampling with skew distribution. Biometrics, 53, 123-130.

[22] Kvam, P. H. and Samaniego, F. J. (1993). On the inadmissibility of empirical averages as estimators in ranked set sampling. Journal of statistical Planning and Inference, 36, 39-55.

[23] Lee, A. J. (1990). U-statistics: theory and practice, Marcel Dekker, Inc.

[24] Lehmann, E. L. (1998). Elements of Large-Sample Theory, New York: springerVerlag.

[25] Li, D. and Chuiv, N. (1997). On the efficiency of ranked set sampling strategies in parametric estimation. Calcutta Statistical Association Bulletin, 47, 23-42.

[26] Lohr, S. L. (1999). Sampling: Design and Analysis, New York: Duxbury. 
[27] MacEachern, S. N., Ozturk, O., Stark, G. and Wolfe, D. A. (2002). A new ranked set sample estimator of variance. Journal of Royal Statistical Society-Series B, 64, 177-188.

[28] MacEachern, S. N., Stasny, E. A., and Wolfe, D. A. (2004). Judgement poststratification with imprecise rankings. Biometrics, 60, 207-215.

[29] McIntyre, G. A. (1952). A method for unbiased selective sampling using ranked sets. Australian Journal of Agricultural Research 3, 385-390.

[30] McIntyre, G. A. (2005). A method for unbiased selective sampling using ranked sets. The American Statistician 3, 230-232. Originally appeared in Australian Journal of Agricultural Research, 3, 385-390.

[31] Mode, N. A., Conquest, L. L., and Marker, D. A. (1999). Ranked set sampling for ecological research: Accounting for the total costs of sampling. Environmetrics 10 (2): 179-194.

[32] Muttlak, H. A. (1996). Estimation of parameters for one-way layout with rank set sampling. Biometrical Journal, 38, 405-515.

[33] Nahhas, R. (1999). Ranked set sampling: Ranking error models, cost, and optimal set size. Ph.D. Dissertation, The Ohio state University.

[34] Nahhas, R., Wolfe, D. A., and Chen, H. (2002). Ranked set sampling: cost and optimal set size. Biometrics, 58, 964-971.

[35] Ozturk, O. (1999a). Two-sample inference based on one-sample ranked-set sample sign statistics. Journal of Nonparametric Statistics, 10, 197-212.

[36] Ozturk, O. (1999b). One- and two-sample sign test for ranked set samples with selective designs. Communications in Statistics, Part A - Theory and Methods, 28,1231-1245.

[37] Ozturk, O. (2002). Rank regression in ranked-set samples. Journal of American Statistical Association, 97, 1180-1191.

[38] Ozturk, O., and MacEachern, S., (2004). Order restricted randomized design for control versus treatment comparison. Annals of Institute of statistical Mathematics, 56, 701-720.

[39] Ozturk, O., and MacEachern, S., (2005). Order restricted randomized designs and two-sample inference. Submitted for publication.

[40] Ozturk, O. and Wolfe, D.A. (2000a). Optimal allocation procedure in ranked set sampling for unimodal and multimodal distributions. Environmental and Ecological Statistics, 7, 343-356. 
[41] Ozturk, O. and Wolfe, D.A. (2000b). An improved ranked-set two-sample MannWhitney-Wilcoxon test. Canadian Journal of Statistics, 28, 123-135.

[42] Ozturk, O., Wolfe, D. A. and Alexandridis, R. (2004). Multi-sample inference for simple-tree alternatives with ranked-set samples. Australian and New Zealand Journal of Statistics, 46, 443-455.

[43] Pierce, D. A. and Peters, D. (1999). Improving on exact tests by approximte conditioning. Biometrika, 86, 265-277.

[44] Perron, F. and Sinha, B. K. (2004). Estimation of variance based on a ranked set sample. J. Statist. Plann. Inf., 120, 21-28.

[45] Ridout, M. S. and Cobby, J. M. (1987). Ranked set sampling with non-random selection of sets and errors in ranking. Applied Statistics, 36, 145-152.

[46] Sinha, B. K. and Purkayastha, S. (1996). On some aspects of ranked set sampling for estimation of normal and exponential parameters. Statist. Decsns., 14, 223240.

[47] Stokes, S. L. (1980). Estimation of variance using judgement ordered ranked set samples. Biometrics, 36, 35-42.

[48] Stokes, S. L. (1995). Parametric ranked set sampling. Annals of the Institute of statistical Mathematics, 47, 465-482.

[49] Stokes, S. L. and Wang, X. (2005). Judgment Post-stratification with Multiple Rankers, Technical Report, Department of Statistical Science, Southern Methodist University.

[50] Takahasi, K. and Wakimoto, K. (1968). On unbiased estimates of the population mean based on the sample stratified by means of ordering. Annals of the Institutes of Statistical Mathematics, 20, 1-31.

[51] Van Der Vaart, A. W. (1998). Asymptotic Statistics. Cambridge University Press. 